Annals of Computer Science and Information Systems Volume 23

\title{
Communication Papers of the 2020 \\ Federated Conference on Computer Science and Information Systems
}

September 6-9, 2020. Sofia, Bulgaria

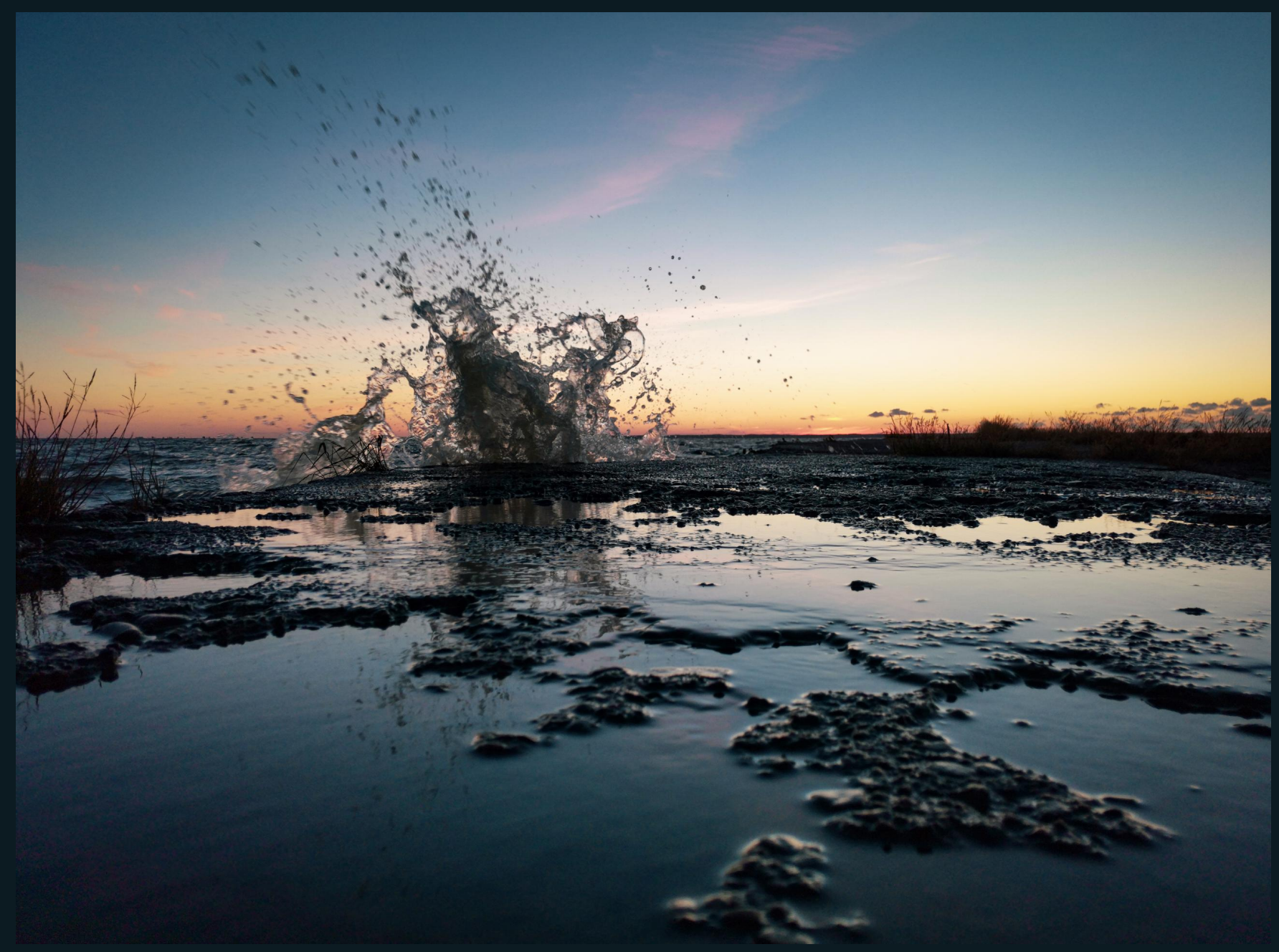

Maria Ganzha, Leszek Maciaszek, Marcin Paprzycki (eds.) 



\section{Annals of Computer Science and Information Systems, Volume 23}

\section{Series editors:}

Maria Ganzha (Editor-in-Chief),

Systems Research Institute Polish Academy of Sciences and Warsaw University of Technology, Poland

Leszek Maciaszek,

Wrockaw Universty of Economy, Poland and Macquarie University, Australia

Marcin Paprzycki,

Systems Research Institute Polish Academy of Sciences and Management Academy, Poland

\section{Senior Editorial Board:}

Wil van der Aalst,

Department of Mathematics \& Computer Science, Technische Universiteit Eindhoven (TU/e), Eindhoven, Netherlands

Enrique Alba,

University of Málaga, Spain

Marco Aiello,

Faculty of Mathematics and Natural Sciences, Distributed Systems, University of

Groningen, Groningen, Netherlands

Mohammed Atiquzzaman,

School of Computer Science, University of Oklahoma, Norman, USA

Jan Bosch,

Chalmers University of Technology, Gothenburg, Sweden

Barrett Bryant,

Department of Computer Science and Engineering, University of North Texas, Denton, USA

Włodzisław Duch,

Department of Informatics, and NeuroCognitive Laboratory, Center for Modern Interdisciplinary Technologies, Nicolaus Copernicus University, Toruń, Poland

Ana Fred,

Department of Electrical and Computer Engineering, Instituto Superior Técnico

(IST-Technical University of Lisbon), Lisbon, Portugal

Janusz Górski,

Department of Software Engineering, Gdansk University of Technology, Gdansk, Poland

Giancarlo Guizzardi,

Free University of Bolzano-Bozen, Italy, Senior Member of the Ontology and Conceptual Modeling Research Group (NEMO), Brazil

Francisco Herrera,

Dept. Computer Sciences and Artificial Intelligence Andalusian Research Institute in Data Science and Computational Intelligence (DaSCI) University of Granada, Spain

Mike Hinchey,

Lero-the Irish Software Engineering Research Centre, University of Limerick, Ireland

Janusz Kacprzyk,

Systems Research Institute, Polish Academy of Sciences, Warsaw, Poland

Irwin King,

The Chinese University of Hong Kong, Hong Kong

Juliusz L. Kulikowski,

Natęc Institute of Biocybernetics and Biomedical Engineering, Polish Academy of Sciences,

Warsaw, Poland

Michael Luck,

Department of Informatics, King's College London, London, United Kingdom 
Jan Madey,

Faculty of Mathematics, Informatics and Mechanics at the University of Warsaw, Poland Stan Matwin,

Dalhousie University, University of Ottawa, Canada and Institute of Computer Science, Polish Academy of Science, Poland

Marjan Mernik,

University of Maribor, Slovenia

Michael Segal,

Ben-Gurion University of the Negev, Israel

Andrzej Skowron,

Faculty of Mathematics, Informatics and Mechanics at the University of Warsaw, Poland John F. Sowa, VivoMind Research, LLC, USA

George Spanoudakis,

Research Centre for Adaptive Computing Systems (CeNACS), School of Mathematics,

Computer Science and Engineering, City, University of London

\section{Editorial Associates:}

Katarzyna Wasielewska,

Systems Research Institute Polish Academy of Sciences, Poland

Paweł Sitek,

Kielce University of Technology, Kielce, Poland

TEXnical editor: Aleksander Denisiuk,

University of Warmia and Mazury in Olsztyn, Poland 


\section{Communication Papers of the 2020 Federated Conference on Computer Science and Information Systems}

Maria Ganzha, Leszek Maciaszek, Marcin Paprzycki (eds.) 
Annals of Computer Science and Information Systems, Volume 23

Communication Papers of the 2020 Federated Conference on Computer Science and Information Systems

USB: $\quad$ ISBN 978-83-959183-3-9

WEB: ISBN 978-83-959183-2-2

ISSN 2300-5963

DOI 10.15439/978-83-959183-2-2

(C) 2020, Polskie Towarzystwo Informatyczne

Ul. Solec 38/103

00-394 Warsaw

Poland

Contact: secretariat@fedcsis.org

http://annals-csis.org/

Cover photo:

Beata Branicka, Elblag, Poland

Also in this series:

Volume 22: Position Papers of the 2020 Federated Conference on Computer Science and Information Systems, ISBN WEB: 978-83-959183-0-8, ISBN USB: 978-83-959183-1-5

Volume 21: Proceedings of the 2020 Federated Conference on Computer Science and Information Systems, ISBN Web 978-83-955416-7-4, ISBN USB 978-83-955416-8-1,

ISBN ART 978-83-955416-9-8

Volume 20: Communication Papers of the 2019 Federated Conference on Computer Science and Information Systems, ISBN WEB: 978-83-955416-3-6, ISBN USB: 978-83-955416-4-3 Volume 19: Position Papers of the 2019 Federated Conference on Computer Science and Information Systems, ISBN WEB: 978-83-955416-1-2, ISBN USB: 978-83-955416-2-9

Volume 18: Proceedings of the 2019 Federated Conference on Computer Science and Information Systems, ISBN Web 978-83-952357-8-8, ISBN USB 978-83-952357-9-5,

ISBN ART 978-83-955416-0-5

Volume 17: Communication Papers of the 2018 Federated Conference on Computer Science and Information Systems, ISBN WEB: 978-83-952357-0-2, ISBN USB: 978-83-952357-1-9 Volume 16: Position Papers of the 2018 Federated Conference on Computer Science and Information Systems, ISBN WEB: 978-83-949419-8-7, ISBN USB: 978-83-949419-9-4

Volume 15: Proceedings of the 2018 Federated Conference on Computer Science and Information Systems, ISBN Web 978-83-949419-5-6, ISBN USB 978-83-949419-6-3, ISBN ART 978-83-949419-7-0

Volume 14: Proceedings of the First International Conference on Information Technology and Knowledge Management, ISBN WEB: 978-83-949419-2-5, ISBN USB: 978-83-949419-1-8, ISBN ART: 978-83-949419-0-1 Volume 13: Communication Papers of the 2017 Federated Conference on Computer Science and Information Systems, ISBN WEB: 978-83-922646-2-0, ISBN USB: 978-83-922646-3-7 Volume 12: Position Papers of the 2017 Federated Conference on Computer Science and Information Systems, ISBN WEB: 978-83-922646-0-6, ISBN USB: 978-83-922646-1-3 
$\mathrm{D}$ EAR Reader, it is our pleasure to present to you Communication Papers of the $15^{\text {th }}$ Conference on Computer Science and Information Systems (FedCSIS'2020), which took place fully remotely, on September 7-9, 2020. Conference was originally planned to take place in Sofia, Bulgaria, but the global COVID-19 pandemics forced us to adapt and organize the conference online.

The communication papers were introduced in 2017 as a separate category of contributions. They report on research topics worthy of immediate communication. They may be used to mark a hot new research territory, or to describe work in progress in order to quickly present it to scientific community. They may also contain additional information omitted from the earlier papers, or may present software tools and products in a research state.

FedCSIS 2020 was Chaired by prof. Stefka Fidanova, while dr. Nina Dobrinkova acted as the Chair of the Organizing Committee. This year, FedCSIS was organized by the Polish Information Processing Society (Mazovia Chapter), IEEE Poland Section Computer Society Chapter, Systems Research Institute Polish Academy of Sciences, Warsaw University of Technology, Wrocław University of Economics and Business, and Institute of Information and Communication Technologies, Bulgarian Academy of Sciences.

FedCSIS 2020 was technically co-sponsored by: IEEE Poland Section, IEEE Czechoslovakia Section Computer Society Chapter, IEEE Poland Section Systems, Man, and Cybernetics Society Chapter, IEEE Poland Section Computational Intelligence Society Chapter, IEEE Poland Section Control System Society Chapter, Committee of Computer Science of the Polish Academy of Sciences, Mazovia Cluster ICT Poland, Eastern Cluster ICT Poland and Bulgarian Section of SIAM.

During FedCSIS 2020, the keynote lectures were delivered by:

- Christian Blum Artificial Intelligence Research Institute (IIIA-CSIC), Barcelona, Spain, "Are you a Hybrid? Yes, of course, everyone is a Hybrid nowadays!"

- George Boustras, European University Cyprus "Critical Infrastructure Protection - on the interface of safety and security"

- Hans-Georg Fill, University of Fribourg, Switzerland, „,From Digital Transformation to Digital Ubiquity: The Role of Enterprise Modeling"

FedCSIS 2020 consisted of five Tracks. Within each Track, topical Technical Sessions have been organized. Some of these Technical Sessions have been associated with the FedCSIS conference series for many years, while some of them are relatively new. Their role is to focus and enrich discussions on selected areas pertinent to the general scope of each Track.

\section{- Track 1: Artificial Intelligence}

- Topical technical sessions:

- $\quad 15^{\text {th }}$ International Symposium on Advanced

Artificial Intelligence in Applications (AAIA'20)

- $\quad 13^{\text {th }}$ International Workshop on

Computational Optimization (WCO'20)

- $\quad 5^{\text {th }}$ International Workshop on Language
Technologies and Applications (LTA'20)

- Track 2: Computer Science \& Systems

- Topical technical sessions:

- $\quad$ Advances in Computer Science and Systems

(ACS\&S'20)

- $\quad 13^{\text {th }}$ Workshop on Computer Aspects of

Numerical Algorithms (CANA'20)

- $\quad 11^{\text {th }}$ Workshop on Scalable Computing

(WSC'20)

- Track 3: Network Systems and Applications

- Topical technical sessions:

- $\quad$ Advances in Network Systems and

Applications (ANSA'20)

- $\quad 4^{\text {th }}$ Workshop on Internet of Things -

Enablers, Challenges and Applications (IoT-

ECAW'20)

- International Forum of Cyber Security,

Privacy, and Trust (NEMESIS'20)

- Track 4: Information Systems and Technology

- Topical technical sessions:

Advances in Information Systems and

Technologies (AIST)

$2^{\text {nd }}$ Special Session on Data Science in

Health, Ecology and Commerce (DSH'20)

- $\quad 15^{\text {th }}$ Conference on Information Systems

Management (ISM'20)

- $\quad 26^{\text {th }}$ Conference on Knowledge Acquisition and Management (KAM'20)

- Track 5: Software and System Engineering

- Topical technical sessions:

- $\quad$ Advances in Software and System

Engineering (ASSE’20)

- $\quad 4^{\text {th }}$ International Conference on Lean and

Agile Software Development (LASD'20)

- $\quad 6^{\text {th }}$ Workshop on Model Driven Approaches in

System Development (MDASD'20)

- Joint $40^{\text {th }}$ IEEE Software Engineering

Workshop (SEW-40) and $7^{\text {th }}$ International Workshop

on Cyber-Physical Systems (IWCPS-7)

Each paper, found in this volume, was refereed by at least two referees.

The program of FedCSIS required a dedicated effort of many people. We would like to express our warmest gratitude to all Committee members, of each Track and each Technical Session, for their hard work in attracting and later refereeing 206 submissions (regular and data mining).

We thank the authors of papers for their great contribution into theory and practice of computing and software systems. We are grateful to the invited speakers for sharing their knowledge and wisdom with the participants.

Last, but not least, we thank prof. Fidanova and dr. Dobrinkova. It should be stressed that they made all the preparations to organize the conference in Bulgaria. They also worked with us diligently when we were forced to move the conference online. Stefka and Nina, we are very grateful for all your efforts! As a matter of fact, we hope to organize FedCSIS in Bulgaria as soon as the World returns to normal (even if it will be the "new normal").

We hope that you had an inspiring conference. We also hope to meet you again for the $16^{\text {th }}$ Conference on Computer Science and Intelligence Systems (FedCSIS 2021). 
Please note an upcoming change in the conference name, from Information Systems to Intelligence Systems. The change is warranted, first, by the changes in the world around us. As can be easily observed, broadly understood, intelligence is permeating all aspects of our reality. Second, this change is already reflected by the kinds of paper submissions that are being received by all FedCSIS Tracks, and our intent to attract even more submissions related to all sorts of Intelligence Systems (including of course Artificial Intelligence, but also Business Intelligence, Management Intelligence, Human Intelligence, Financial Intelligence, Embedded Intelligence, Computational Intelligence, Collective Intelligence, Biomedical Intelligence, Military Intelligence, Network Intelligence...).
Taking into account the level of uncertainty related to COVID-19, we are seriously considering organizing the next edition of the conference online, again. However, the final decision has not been reached, yet.

\section{Co-Chairs of the FedCSIS Conference Series}

Maria Ganzha, Warsaw University of Technology, Poland and Systems Research Institute Polish Academy of Sciences, Warsaw, Poland

Leszek Maciaszek, Wrocław University of Economics, Wroctaw, Poland and Macquarie University, Sydney, Australia

Marcin Paprzycki, Systems Research Institute Polish Academy of Sciences, Warsaw Poland and Management Academy, Warsaw, Poland 



\section{Communication Papers of the 2020 Federated Conference on Computer Science and Information Systems (FedCSIS)}

September 6-9, 2020. Sofia, Bulgaria

TABLE OF CONTENTS

\section{ARTifiCial INTELLigenCe}

\section{$13^{\text {TH }}$ INTERNATIONAL WORKSHOP ON COMPUTATIONAL}

\section{OPTIMIZATION}

\section{Call For Papers}

Energetic Optimization of the Use of Battery Shunting Locomotive in Industrial Plant with Regenerative Brake

Tsvetomir Gotsov, Venelin Todorov, Tsvetelin Kolev

An Iterative Descent Method for Predicting The Compressive Cement Strength Estimated

Parameters

Mhand Hifi, Adeline Goullieux, Shohre Sadeghsa

An Optimization on Quadrature Formulas and Numerical Solutions of Ordinary

Differential Equations

Venelin Todorov, Yuri Dimitrov, Radan Miryanov, Ivan Dimov, Stefka Fidanova, Stoyan Poryazov

An Optimal Monte Carlo Algorithm for a Class of Multidimensional Integrals

Venelin Todorov, Ivan Dimov, Stoyan Apostolov, Stefka Fidanova, Stoyan Poryazov, Yuri Dimitrov

A New Optimized Stochastic Approach for Multiple Integrals in Option Pricing

Venelin Todorov, Ivan Dimov, Stefka Fidanova, Stoyan Poryazov

Optimized Quasi-Monte Carlo Method Based on Low Discrepancy Sequences for

Sensitivity Analysis in Air Pollution Modelling

Venelin Todorov, Tzvetan Ostromsky, Ivan Dimov, Stefka Fidanova

Sensitivity Study of a Large-Scale Air Pollution Model by Using Optimized Stochastic Algorithm

Venelin Todorov, Tzvetan Ostromsky, Ivan Dimov, Stefka Fidanova

\section{NETWORK SYSTEMS AND APPLICATIONS}

\section{ADVANCES IN NETWORK SYSTEMS AND APPLICATIONS}

Call For Papers
Direct communication of edge elements in the Industrial Internet of Things

Piotr Hajder, Mirosław Hajder, Mateusz Liput, Mariusz Nycz 
$1^{\text {St }}$ International Forum on Cyber Security, Privacy And

TRUST

Call For Papers

The general universal model of blockchain technology based on an analysis of some implementations

Polina Sazonova

INFORMATION SYSTEMS AND TECHNOLOGY

$2^{\text {ND }}$ Special Session on Data Science in Health, Ecology and

COMMERCE

Call For Papers

Interactive Online Reporting of Registry of Tuberculosis

Matěj Karolyi, Tereza Nováková, Petr Panoška, Jiří Wallenfels, Martin Komenda, Jiří Jarkovský

Conceptualization of an AI Based Assistant to Support the Compatibility of Family and

Paid Work

Philippe Krajsic, Martin Schieck, Ingolf Römer, Silvia Torres Landaverde, Carsta Militzer-Horstmann, Bogdan Franczyk

SOFTWARE AND SYSTEM ENGINEERING

JOINT 40 ${ }^{\text {TH }}$ IEEE SOFTWARE ENGINEERING WORKSHOP AND 7TH INTERNATIONAL WORKSHOP ON CYBER-PHYSICAL SYSTEMS

Call For Papers

Capturing the Evolution of Service-oriented Systems with Architectural Decisions

Szymon Kijas, Andrzej Zalewski

Author Index 


\section{$13^{\text {th }}$ International Workshop on Computational Optimization}

$\mathbf{M}$ ANY real world problems arising in engineering, economics, medicine and other domains can be formulated as optimization tasks. These problems are frequently characterized by non-convex, non-differentiable, discontinuous, noisy or dynamic objective functions and constraints which ask for adequate computational methods.

The aim of this workshop is to stimulate the communication between researchers working on different fields of optimization and practitioners who need reliable and efficient computational optimization methods.

\section{TOPICS}

The list of topics includes, but is not limited to:

- combinatorial and continuous global optimization

- unconstrained and constrained optimization

- multiobjective and robust optimization

- optimization in dynamic and/or noisy environments

- optimization on graphs

- large-scale optimization, in parallel and distributed computational environments

- meta-heuristics for optimization, nature-inspired approaches and any other derivative-free methods

- exact/heuristic hybrid methods, involving natural computing techniques and other global and local optimization methods

- numerical and heuristic methods for modeling

The applications of interest are included in the list below, but are not limited to:

- classical operational research problems (knapsack, traveling salesman, etc)

- computational biology and distance geometry

- data mining and knowledge discovery

- human motion simulations; crowd simulations

- industrial applications
- optimization in statistics, econometrics, finance, physics, chemistry, biology, medicine, and engineering

- environment modeling and optimization

\section{Best PAPER AWARD}

The best WCO'20 paper will be awarded during the social dinner of FedCSIS 2020.

The best paper will be selected by WCO' 20 co-Chairs by taking into consideration the scores suggested by the reviewers, as well as the quality of the given oral presentation.

\section{TeChnical SEssion CHAIRS}

- Fidanova, Stefka, Bulgarian Academy of Sciences, Bulgaria

- Mucherino, Antonio, INRIA, France

- Zaharie, Daniela, West University of Timisoara, Romania

\section{Program CommitTee}

- Abud, Germano, Universidade Federal de Uberlândia, Brazil

- Bonates, Tibérius, Universidade Federal do Ceará, Brazil

- Breaban, Mihaela

- Gruber, Aritanan

- Hadj Salem, khadija, University of Tours - LIFAT Laboratory, France

- Hosobe, Hiroshi, Hosei University, Japan

- Lavor, Carlile, IMECC-UNICAMP, Brazil

- Micota, Flavia, West University of Timisora, Romania

- Muscalagiu, Ionel, Politehnica University Timisoara, Romania

- Stoean, Catalin, University of Craiova, Romania

- Wang, Yifei

- Zilinskas, Antanas, Vilnius University, Lithuania 



\title{
Energetic optimization of the use of battery shunting locomotive in industrial plant with regenerative brake
}

\author{
Tsvetomir Gotsov $\ddagger$, Venelin Todorov*广 ${ }^{* \dagger}$ Tsvetelin Kolev ${ }^{\S}$ \\ *Institute of Mathematics and Informatics \\ Bulgarian Academy of Sciences \\ 8 Acad. G. Bonchev Str., 1113 Sofia, Bulgaria \\ $\dagger$ Institute of Information and Communication Technologies \\ Bulgarian Academy of Sciences \\ 25A Acad. G. Bonchev Str., 1113 Sofia, Bulgaria \\ $\ddagger$ Electronics Department, University of Ruse, Bulgaria

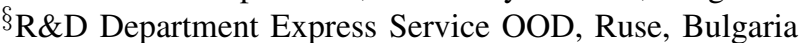 \\ Email: vtodorov@math.bas.bg, venelin@parallel.bas.bg, tsvetomir.gotsov@gmail.com, info@1z1866.com
}

\begin{abstract}
Shunting locomotives perform specific functions characterized by short mileages, variable load weights and a high idling rate. Battery technology based on traction is a very suitable solution for shunting locomotives due to the possibility of accumulating kinetic energy in the power supply. The optimization of the energy efficiency of regenerative braking and the possibilities for efficient shunting in industrial plant were studied.
\end{abstract}

\section{INTRODUCTION}

Maneuvering activity is a major operation performed on the territory of freight yards, in passengers terminal stations, depots, in industrial plants connected to rail network, in harbours and multimodal terminals. Industrial sites connected to the rail network own shunter locomotives for freight wagons shunting on their premises. In order to be able to operate independently from the overhead voltage, or on non-electrified rail, most shunter locomotives are diesel electric or diesel hydraulic locomotives [1]. Diesel engines typically operate in an inefficient mode, which in a competitive business environment makes them economically unprofitable. To a great extent, this is aided by the rising fuel prices over the past decade and the high maintenance costs of conventional engines. In the case of battery-powered locomotives, these costs are reduced to the maintenance costs of the used battery. Machines on batteries have the possibility of regenerative braking, which enables the kinetic energy to be transformed into electric and re-accumulated in the battery [12]. Interesting is the energy efficiency of this type of braking and its benefits for shunting locomotives.

Venelin Todorov is supported by KP-06-M32/2-17.12.2019 'Advanced Stochastic and Deterministic Approaches for Large-Scale Problems of Computational Mathematics" and by the National Scientific Program "Information and Communication Technologies for a Single Digital Market in Science, Education and Security" (ICTinSES), contract No. D01-205/23.11.2018, financed by the Ministry of Education and Science.

\section{OPERATION OF SHUNTING LOCOMOTIVES}

There is clearly a very great diversity of shunting duties depending from local railways configuration and practices. But it is possible to identify some common characteristics:

- Shunting is operated at low speed, typically below $15 \mathrm{~km} / \mathrm{h}$.

- The distance traveled with the shunted vehicle or train is relatively short less than $5 \mathrm{~km}$

- The loads to shunt vary in a wide range from the mass of a shunting locomotive to several thousand tons.

When maneuvering with train braking or unloading special types of wagons, large amounts of compressed air are required, which requires the use of powerful air compressors in this type of locomotive. A special feature of the shunting movements is that the locomotive does not leave its place of work and in a large percentage of cases it has access to standard industrial voltage. This feature of shunting locomotives allows the use of batteries tailored to a single shift for a particular application and a minimum volume and power of the locomotive.

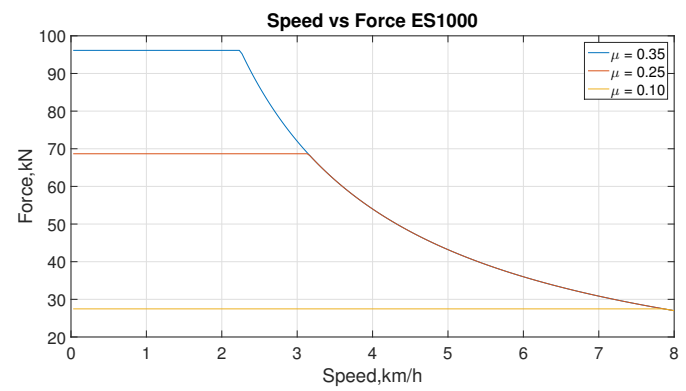

Fig. 1. Traction characteristics ES1000 Shunting locomotive 


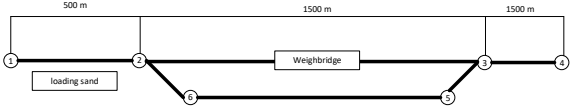

Fig. 2. Railway from industrial plant

The track development of an industrial plant for loading chamotte shows Fig 2. In section 1-2 carriage of wagons is carried out by laying on each wagon.

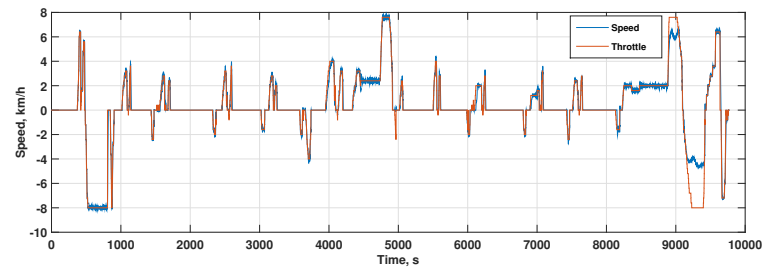

Fig. 3. Typical speed mode when loading 12 freight wagons with chamotte in industrial plant.

The mode at which this task is performed can be seen in Fig. 3 in the range $1000-8000$ s. After filling all the wagons, the composition passes through the Weighbridge in section 23 and interval 8000-9000s. Fig. II. Continue to section 3-4 for a change of the rail. In section 5-6 there is a stay of full wagons, ready to travel on national infrastructure. After the interval 8000 s. in Fig. 3 is the hardest job of the locomotive in which it draws $960 t$. The diagram in Fig. 3 shows: in orange the set speed reference and in blue the current engine speed for the entire period of the study. Negative speed marks a backward movement.

\section{THE SYSTEM UNDER STUDY}

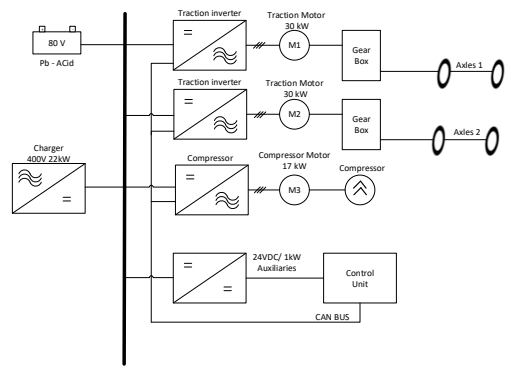

Fig. 4. Electrical diagram from battery shunting locomotive.

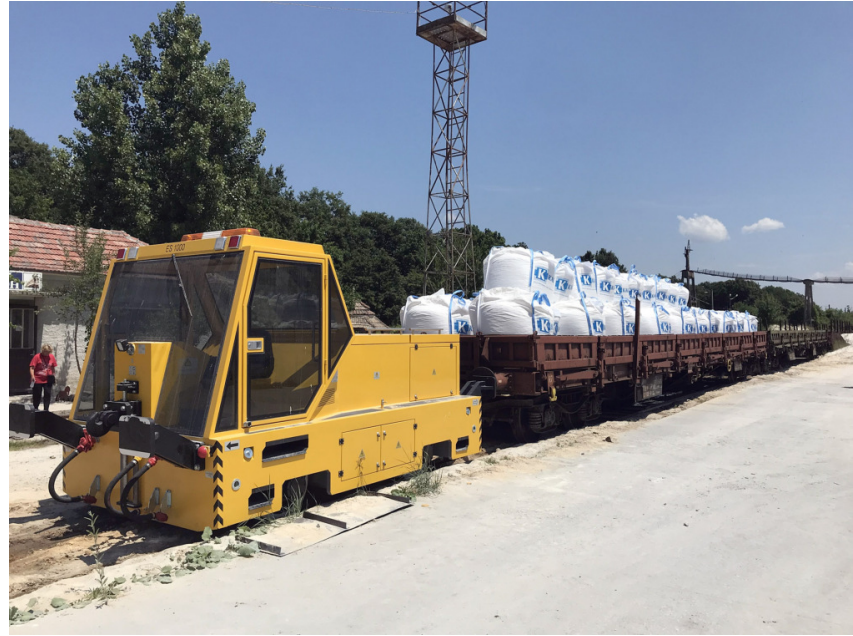

Fig. 5. ES1000 in working conditions.[2]

Shunting locomotives differ from highway locomotives with the lower power and maximum speed they possess. Battery Shunting locomotives in turn differ with extremely low power due to technological disadvantages of modern sources for energy storage, namely, low density and massive mass. Schematic diagram of an ES1000 shunting locomotive represented in Fig. 4. The locomotive has two axles and has a total weight of $28 t$. Each wheel is powered by Induction Motor with a power of $30 \mathrm{~kW}$. There is also an Induction Motors of $17 \mathrm{~kW}$ to drive the air compressor. Control of the electric motors is carried out by a four quadrant vector control. As a power source, Lead-Acid Battery with a capacity of $1356 \mathrm{Ah}$ and a voltage of $80 \mathrm{VDC}$ is used. The total stored power of the locomotive equals $390 \mathrm{MJ}$, sufficient to provide energy for one work shift. Battery is the oldest electric energy stored technology. A battery consists of multiple electrochemical cells, connected in parallel and series to form a unit. Cells consist of two electrodes immersed in an electrolyte solution. Batteries work based on the following principle: due to reversible chemical reactions that occur at the electrodes, a potential difference appears between them. Consequently, energy can reversibly change from electrical form to the chemical form [4], [14], [15]. The Lead-Acid battery is unpretentious to the charge current and for short periods can resist larger circuit currents. Charge of the battery from the power grid takes 5 hours, from a fully discharged battery. The maximum discharge current of the traction inverter is $650 \mathrm{~A}$ each. The maximum allowed discharge current of the entire system is:

$$
\begin{gathered}
I_{\text {dischargemax }}=I_{\text {traction } 1}+I_{\text {traction } 2}+I_{\text {comp. }}+I_{\text {aux. }}= \\
=650+650+150+50 \approx 1500 \mathrm{~A}
\end{gathered}
$$

Current with this value is peak and it lasts less than a minute. The control is carried out by the Control Unit and the distributed system from the traction controllers. All electronics 
device connected in CAN-bus network. Data capture is done via the Serial port of the traction inverter. Data is recorded by an additional installed logger on the shunting locomotive with a sampling time of $0.5 \mathrm{~s}$. Data recording begins immediately after the locomotive exits the Depot for the interval 0-1000s /Fig. 3/ and ends at Depot's place for charge. Locomotive in the experimental conditions of an industrial plant shows in Fig. 5.

\section{Results}

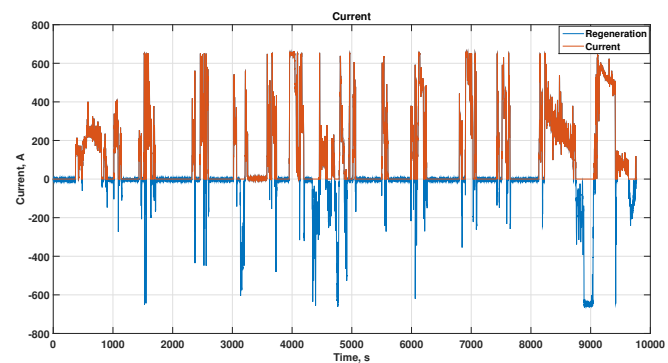

Fig. 6. Traction current $\log$ from traction inverter 1

The experiment was carried out in an industrial plant for loading chamotte. Fig. 6 shows a current log consumed by $M 1$ for one working cycle $(t \approx 10000 s$. ). The orange graph is the consumed current, and the blue one is the current returned to the battery, marked with a reverse sign. In the conducted trials, the train stop is performed only by a traction engine. The train brake is switched on but not in use. Its goal is to increase the security of shunting.

The stored kinetic energy $E_{k}$ during movement with a $960 t$ train and a speed of $8 \mathrm{~km} / \mathrm{h}$ is:

$$
E_{k}=\frac{m v^{2}}{2}=\frac{960 t(8 \mathrm{~km} / \mathrm{h})^{2}}{2}=4,7 \mathrm{MJ}
$$

In case of braking, this energy must be scattered into the atmosphere in the form of heat or re-accumulated in the form of electrical energy. Modern propulsion technologies enable much of this energy to be absorbed and reused. These capabilities of electric locomotives and especially battery locomotives create the opportunity to increase energy efficiency in rail transport. The change in battery voltage is illustrated in Fig. 7. The nominal voltage is $80 \mathrm{~V}$ but due to the large discharge currents, it drops to $55 \mathrm{~V}$ at discharge and increases to $95 \mathrm{~V}$ in the regeneration brake and charge from the Traction inverter.

The energy consumed without regenerative braking for the shunting cycle:

$$
E_{\text {brake }}=\int_{0}^{T} u(t) i(t) d t=85.5 \mathrm{MJ}
$$

The energy consumed with regenerative braking for the shunting cycle:

$$
E_{\text {regbrake }}=\int_{0}^{T} u(t) i(t) d t=60.7 M J
$$

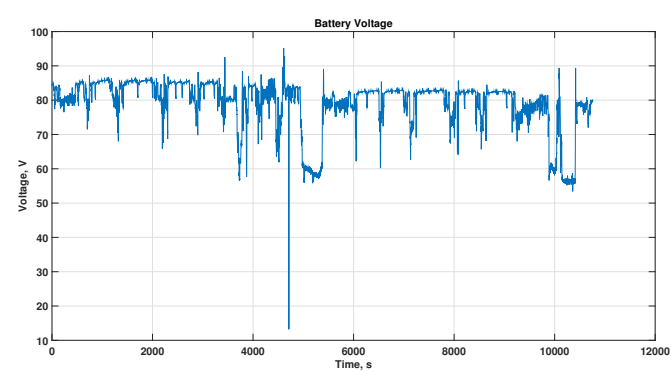

Fig. 7. Voltage drop for Lead -Acid battery

Energy efficiency from the use of regenerative braking:

$$
E E=\left(\frac{E_{\text {brake }}}{E_{\text {regbrake }}}-1\right) * 100=41 \%
$$

The research is done from recorded data on one of the traction inverters. The total power consumption of the two drives and the air compressor is $219 M J(61 \mathrm{kWh})$ measured as the electricity required to charge the battery from the power grid. For the same amount of work done with a diesel locomotive, the energy consumed equals 80 liters of diesel fuel. The data for about $41 \%$ efficiency is also confirmed by other publications related to the study of regenerative braking energy efficiency [3-8,17-25]. The one-year electricity savings during similar work operations from the use of a regenerative brake is approximately:

$$
E_{\text {reg }}=E_{\text {brake }}-E_{\text {regbrake }}=6000 \mathrm{MJ}=1.6 \mathrm{MW} \mathrm{W} / \mathrm{h}
$$

The economic benefit of using a battery shunting locomotive instead of manoeuvrable diesel engine is the electricity consumption for a year /instead of diesel fuel/:

$$
E_{\text {total }}=(240 \text { workingdaysright })(61 \mathrm{~kW} / \mathrm{h})=14640 \mathrm{~kW} / \mathrm{h}
$$

Diesel fuel consumption per year for the same type of work is:

$$
\text { Dieselfuel }=(240 \text { workingdays })(80 L)=19200 L
$$

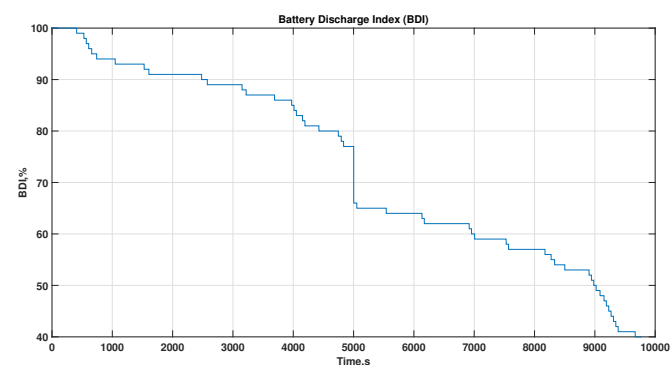

Fig. 8. Battery discharge index for Lead-Acid 
Consumption of energy from the battery illustrates Fig. 8 . It could be seen that for the given work cycle, loading and shunting of 12 chamotte wagons, the battery has rendered all of its energy up to 40 .

For larger volumes of work, the capacity of the battery can be increased in accordance with a particular application. The locomotive works during different periods of the year under different climatic conditions in a moderate climate with success and without failures or change in performance. At ambient temperatures of $-20 \mathrm{C}$, changes in battery capacity do not have a significant effect on the performance. Despite the small number of Lead-Acid Battery cycles and shorter lifetime than alternative options, their life may exceed 10 years in normal exploitation. At this price point these type of batteries used in vehicles remain the cheapest solution [9], [20].

\section{CONCLUSION}

This article explores the energy efficiency optimization of regenerative braking in shunting locomotives instead of the conventional braking with the automatic train brake. LeadAcid technology has been studied to power and store energy. Regenerative braking optimizes shunting costs and reduces wear on train braking systems. Electric battery shunting locomotives provide a real opportunity to reduce emissions in cities and industrial plants. The reduction of harmful emissions in railway transport has emerged as a strategic task for the Union of Railways with a database of technologies enabling optimization of the energy efficiency. The ability to charge the battery from renewable energy sources allows us to achieve zero emission. In the future work some other techniques for energy optimization will be discussed.

\section{REFERENCES}

[1] http://www.railway-research.org/IMG/pdf/r.2.2.3.1.pdf (accessed on May 2019)

[2] http://lz1866.com/battery-locomotives\#es (accessed on 2 May 2019)

[3] M. Ogasa, Energy saving and environmental measures in railway technologies: Example with hybrid electric railway vehicles, IEEJ Trans. Electr. Electron. Eng., vol. 3, no. 1, pp. 15-20, 2008 DOI: $10.1002 /$ tee. 20227

[4] M. Khodaparastan, A. A. Mohamed, and W. Brandauer, Recuperation of regenerative braking energy in electric rail transit systems, IEEE Trans. Intell. Transp. Syst., vol. 20, no. 8, pp. 2831-2847, 2019. DOI:10.1109/TITS. 2018.2886809

[5] R. Takagi, Energy saving techniques for the power feeding network of electric railways, IEEJ Trans. Electr. Electron. Eng., vol. 5, no. 3, pp. 312-316, 2010. DOI:10.1002/tee.20535

[6] M. Shimada, R. Oishi, D. Araki, and Y. Nakamura, Energy storage system for effective use of regenerative energy in electrified railways, Hitachi Rev., vol. 59, no. 1, pp. 33-38, 2010. ISSN 0018277X

[7] M. Saleh, O. Dutta, Y. Esa, and A. Mohamed, Quantitative analysis of regenerative energy in electric rail traction systems, 2017 IEEE Ind. Appl. Soc. Annu. Meet. IAS 2017, vol. 2017-Janua, pp. 1-7, 2017. R. Nicole, Title of paper with only first word capitalized, J. Name Stand. Abbrev., in press. DOI:10.1109/IAS.2017.8101774
[8] Available online: https://www.alstom.com/sites/alstom.com/files/2018/ 10/30/re-use_laymans_report_en.pdf (accessed on 9 May 2019).

[9] UIC, Energy Efficiency Technologies for Railways. Available online: http://www.railway-energy.org (accessed on 9 May 2019).

[10] M. Steiner and J. Scholten, Energy storage on board of railway vehicles, 2005 Eur. Conf. Power Electron. Appl., vol. 2005, pp.1-10, 2005. DOI: $10.1002 /$ tee. 20535

[11] R. Takagi, Application of energy storage systems for DC electric railways, WIT Trans. Ecol. Environ., vol. 121, pp. 527-535, 2009. DOI: $10.2495 / E S U 090481$

[12] X. Luo, J. Wang, M. Dooner, and J. Clarke, Overview of current development in electrical energy storage technologies and the application potential in power system operation, Appl. Energy, vol. 137, pp. 511536, 2015. DOI:10.1016/j.apenergy.2014.09.081

[13] A. Gonzalez-Gil, R. Palacin, and P. Batty, Sustainable urban rail systems: Strategies and technologies for optimal management of regenerative braking energy, Energy Convers. Manag., vol. 75, pp. 374-388, 2013. DOI:10.1016/j.enconman.2013.06.039

[14] W. Gunselmann, Technologies for increased energy efficiency in railway systems, 2005 Eur. Conf. Power Electron. Appl., vol. 2005, pp. 1-10, 2005. DOI:10.1109/epe.2005.219712

[15] J. Hu, Y. Zhao, and X. Liu, The design of regeneration braking system in light rail vehicle using energy-storage Ultra-capacitor, 2008 IEEE Veh. Power Propuls. Conf. VPPC 2008, pp. 1-5, 2008.DOI:10.1109/VPPC.2008.4677708

[16] M. Miyatake and K. Matsuda, Energy saving speed and charge/discharge control of a railway vehicle with on-board energy storage by means of an optimization model, IEEJ Trans. Electr. Electron. Eng., vol. 4, no. 6, pp. 771-778, 2009. DOI:10.1002/tee.20479

[17] European Commission, Electrification of the Transport System: Studies and Reports, pp. 1-49, 2017. ISBN:9789279718564

[18] A. Fernandez-Rodriguez et al., Charging Electric Vehicles Using Regenerated Energy from Urban Railways, 2017 IEEE Veh. Power Propuls. Conf. VPPC 2017 - Proc., vol. 2018-January, pp. 1-6, 2018. DOI:10.1109/VPPC.2017.8330998

[19] K. Ogura et al., Test results of a high capacity wayside energy storage system using Ni-MH batteries for DC electric railway at New York City Transit, 2011 IEEE Green Technol. Conf. Green 2011, pp. 6-11, 2011. DOI: 10.1109/GREEN.2011.5754859

[20] M. Ceraolo and G. Lutzemberger, Stationary and on-board storage systems to enhance energy and cost efficiency of tramways, J. Power Sources, vol. 264, pp. 128-139, 2014. DOI:10.1016/j.jpowsour.2014.04.070

[21] D. Iannuzzi and P. Tricoli, Speed-based state-of-charge tracking control for metro trains with onboard supercapacitors, IEEE Trans. Power Electron., vol. 27, no. 4, pp. 2129-2140, 2012. DOI:10.1109/TPEL.2011.2167633

[22] D. Iannuzzi and P. Tricoli, Supercapacitor state of charge control based on changeover finite state controller for metro-train applications, 3rd Int. Conf. Clean Electr. Power Renew. Energy Resour. Impact, ICCEP 2011, pp. 550-556, 2011.

[23] L. Mir, I. Etxeberria-Otadui, I. P. De Arenaza, I. Sarasola, and T. Nieva, A supercapacitor based light rail vehicle: System design and operations modes, 2009 IEEE Energy Convers. Congr. Expo. ECCE 2009, vol. 146, pp. $1632-1639$, 2009. DOI:10.1109/ECCE.2009.5316073

[24] M. Steiner and J. Scholten, Energy storage on board of railway vehicles, 2005 Eur. Conf. Power Electron. Appl., vol. 2005, pp. 1-10, 2005 DOI: $10.1109 /$ epe. 2005.219410

[25] D. Grimaud and J. Levraut, Optimal driving strategy for traction energy saving on DC suburban railways, Minerva Anestesiol., vol. 67, no. 4, pp. 263-270, 2001.DOI:10.1049/iet-epa 


\title{
An Iterative Descent Method for Predicting The Compressive Cement Strength Estimated Parameters
}

\author{
Adeline Goullieux, Mhand Hifi, Shohre Sadeghsa \\ EPROAD, Université de Picardie Jules Verne \\ 7 rue du Moulin Neuf, 80000 Amiens, France \\ Emails: Adeline.goullieux@u-picardie.fr, hifi@u-picardie.fr, shohre.sadeghsa@u-picardie.fr
}

\begin{abstract}
In this paper, we propose an iterative descent method to predict compressive cement strength estimated parameters for lime and cement as coating substances. We first propose a formal description of the problem by using a mathematical model that is based upon a series of equations. The aforementioned equations are related to both the ratio of quantity of water to quantity of coating substance and the ratio of quantity of straw to quantity of coating substance. Second, we propose to solve the model by applying a gradient descent method. It is applied for reaching results closest to the data gathered from a real experimental studies conducted on the coating of flax straw before incorporating them into a cement matrix. The experimental part shows that the proposed model is capable to predict The the estimation of the parameters necessary for such study.
\end{abstract}

Index Terms-Prediction; Optimization; Gradient descent.

\section{INTRODUCTION}

T HE MOST important mechanical property of a concrete is it's compressive strength. Compressive strength test is a commonly used experiment to measure the characteristics of the cement properties. In this study the experiment is conducted in the laboratory of IMAP on the coating of flax straw before their incorporation into a cement matrix. During the experiment, the ratio of quantity of water to quantity of Coating Substance (noted CS) and the ratio of the quantity of straw to quantity of CS varied.

Measured characteristics are density, water absorption capacity and decrease in water absorption capacity (comparison with untreated straws) of straws and flexural strength, compressive strength, density, dimensional variation on drying and extreme dimensional variation of composites. The objective of the experiment is to have high mechanical properties and low dimensional variation on the formulated product.

Several studies proposed soft computing-based approaches to predict the mechanical strength of concrete. Generally, these approaches tend to design models based upon linear and / or nonlinear regression equations (cf., Snell, et al. [1]).

During practical sessions, we noticed that this experiment is time consuming and expensive as it needs to recruit people to perform the experiments. In order to overcome these difficulties, often encountered in these studies, we propose a mathematical model for predicting results related to the principal parameters of the study.

The rest of the paper is organized as follows. Section II describes a brief overview of the related studies. In section III, the parameters of the experiment is described, where a brief representation of the data used is provided in section IV. Section V shows how linear regression-based method can be applied for the considered study. Finally, the proposed equations are presented in section VI and the last section VII concludes the paper by summarizing the contents of the study and future studies.

\section{AN OVERVIEW OF THE LITERATURE}

Compressive Cement Strength Test (namely CCST) is the most important test for quality control which conventionally takes 28 days of experiments (for more details the reader can be referred to de Siqueira Tango [2]).

Generally there are two ways to perform CCST: (i) the first way can be achieved by considering the experimental test, where Accelerated Atrength test methods (namely AST) is applied and, (ii) the second way based on predicting the variables-values using soft computing-based methods.

CCST by itself is time consuming and therefore expensive for industries. Considering the importance of CCST, we decided to simulate the experiment by proposing mathematical formulations. Indeed, we mainly propose equations in this study that are able to simulate the variables of CCST.

We note that there are several studies that focus on the soft computing-based methods, including regressions and neural networks. However regression methods seems popular and less complicated to tackle when compared to the other studies (cf., de Siqueira Tango [2] and, Snell, et al. [1]).

Behavior of these materials by itself depends on several chemical and physical parameters that affect each other in nature. Therefore, detecting and optimizing these materials is not the most efficient method. Thus, both statistical and analytical models with the use of soft computing-based approaches, including regression analysis seems a promising way to the Compressive Cement Strength Prediction Problem (noted CCSPP).

The optimization of concrete (and generally composites) concerns selecting values for the constituent parameters. Baykasoglu et al. [4] proposed a two-step approach for multi objective optimization of high-strength concretes (namely HSCs). In their study, they applied a regression analysis, a neural networks and a gen expression programming to predict HSCs parameters in the first step. The first step established equations that represent concrete characteristic in terms of its components. According to these equations, a multiobjective optimization model has been proposed that is solved by using a genetic algorithm; that is the second step of their approach.

In Baykasoglu et al. [3] another way to tackle the CCSPP has been proposed. Indeed, the authors proposed a gene 
expression programming, neural networks and stepwise regression analysis as soft computing techniques.

Herein, we aim to simulate CCST by mathematical equations. The equations will return the parameters that needs to be varied in a real experiment. Results of the equations obtained in this study can be replaced with carried experiment.

\section{COMPRESSIVE CEMENT STRENGTH TEST PARAMETERS}

In this study we considered two different experiments:

1) The first experiment. The objective is to predict the ratio of quantity of water to quantity of CS and the ratio of quantity of straw to quantity of CS for lime by having the density, water absorption capacity and decrease in water absorption capacity (comparison with untreated straws) of straws and flexural strength, compressive strength, density, dimensional variation on drying and extreme dimensional variation of composites. This experiment will results two equation where each of this equations is used to predict a ratio.

2) The second experiment. In addition to the first experiment, the second experiment will calculate the two ratios related to the cement.

Table I reports the parameters used in this study. These parameters are considered in both experiments for Lime and Cement.

TABLE I: Design variables

\begin{tabular}{l|l} 
Symbol & Parameter \\
\hline X1 & Shive water absorbance diminution (\%) \\
X2 & Shive water absorbance $(\%)$ \\
X3 & Shive bulk density $(\mathrm{kg} / \mathrm{m} 3)$ \\
X4 & Composite Rf $(\mathrm{Mpa})$ \\
X5 & Composite Rc (MPa) \\
X6 & Composite bulk density $(\mathrm{kg} / \mathrm{m} 3)$ \\
X7 & Composite DS $(\mathrm{mm} / \mathrm{m})$ \\
X8 & Composite EDV $(\mathrm{mm} / \mathrm{m})$ \\
\hline Y1 & Shive/Coating substance (S/CS) \\
Y2 & Water/Coating Substance (W/CS) \\
\hline
\end{tabular}

Therefore, using symbols introduced in Table I, on the one hand, for the first experiment the parameters are represented by the set $\{\mathrm{X} 1, \mathrm{X} 2, \mathrm{X} 3, \mathrm{X} 4, \mathrm{X} 5, \mathrm{X} 6, \mathrm{X} 7, \mathrm{X} 8\}$ and $\mathrm{Y} 1$ denotes the variable to predict for Lime. On the other hand, for the second equation the parameters are characterized with the set $\{\mathrm{X} 1, \mathrm{X} 2, \mathrm{X} 3, \mathrm{X} 4, \mathrm{X} 5, \mathrm{X} 6, \mathrm{X} 7, \mathrm{X} 8\}$ and $\mathrm{Y} 2$ is the predicted variable. In the second experiment the similar parameters are selected using data of Cement.

\section{DATA COLLECTION}

Practical experiments are conducted in IMAP Laboratory, where 123 experiments were conducted on the coating substances including: (i) 60 experiments on Lime, (ii) 3 on the non treated material and, (iii) 60 experiments on Cement.

In the experiment, for each $\mathrm{CS}$, the ratio of quantity of water to quantity of $\mathrm{CS}$ is varied in the interval $\{0.33,0.5,0.75$, 1 ) while the ratio of the quantity of straw to quantity of CS is varied in interval $\{0.5,0.751,2\}$.
For each CS the measured parameters are shive water absorbance (\%), shive bulk density ( $\mathrm{kg} / \mathrm{m} 3)$, composite $\mathrm{Rf}$ (Mpa), composite Rc (MPa), composite bulk density (kg/m3), composite DS $(\mathrm{mm} / \mathrm{m})$, composite EDV $(\mathrm{mm} / \mathrm{m})$. Finally, the amount of shive water absorbance diminution (\%) is mathematically calculated using the provided data from the experiments.

We note that the goal of the experiment is to preserve high mechanical properties and low dimensional variation on the formulated coating substances. Average of the data for each experiment is summarized in Table III

\section{REGRESSION ANALYSIS}

There are several studies in the literature that implement various algorithms to optimize gradient descent as one of the most popular optimization methods.(e.g. Bengio [6])

The gradient descent my be distinguished with its three variants: (i) batch gradient descent, (ii) stochastic gradient descent and, (iii) mini-batch gradient descent. The difference between these varaints lies in the amount of the data.

Herein, as mentioned in below, data is expensive and collecting the data is time consuming. Therefore, the amount of available data are limited.

Batch gradient descent calculates the gradient for the whole data set. Therefore, dealing with large dataset, batch gradient descent performs very slowly. Because the number of data are limited in this study, we can safely use the batch gradient descent (for more details, the reader can refer to Ruder [5].

\section{A. Gradient descent}

One of the most applied methods in the literature, to perform optimization and prediction, we can cite the gradient descent; that is due to its simplicity and applicability for several applications and problems to solve.

In what follows, the aim is to establish linear equations to predict CCST parameters. Indeed, the proposed linear model may be formulated as follows:

$$
P(\theta)=\theta_{0}+X \theta
$$

In equation (1), $\theta_{0}$ refers to a constant value, $X$ deotes the parameters of the model and $\theta$ is the coefficient of the parameters. The objective function is defined as the average summation of the square prediction errors for the linear regression. Differently stated, the objective function is also referred as the following cost function:

$$
C(\theta)=\frac{1}{2 m} \sum_{i=1}^{m}\left(P(\theta)_{i}-Y_{i}\right)^{2},
$$

where $\mathrm{C}(\theta)$ represents the cost function for each $\theta, m$ denotes the number of the training examples and $Y$ is the actual value of the variable; that is predicted using the cost function.

The gradient descent tries to minimize the objective function using an iterative procedure, where the coefficients of the 
parameters of the model are updated by using the following equation:

$$
\theta_{j}:=\theta_{j}-\frac{\lambda}{m} \sum_{i=1}^{m}\left(P(\theta)_{i}-Y_{i}\right) X_{i}
$$

At each iteration of the gradient descent, $\theta$ will be updated to the opposite direction of the gradient of the objective function. The parameter $\lambda$ determines the learning rate. It determines step sizes to reach a local minimum. In other words, at each step we move to the direction of the slope of the objective function towards the bottom of a slope, until a local minimum is reached.

\section{B. Principal analysis}

Data described and explained in section IV are analyzed by using the following procedure:

1) Shuffling data.

As described in section IV, data are organized during experimental test. Thus, we shuffled the data to avoid miscalculations and computational errors. The shuffling is performed in a way that only the order of the data will be permuted randomly not the parameters of the experiments.

2) Separate dataset into training set and test set:

Data is separated into two groups: the training set and the test set. Shuffling the data in the previous step is necessary so that there will be no difference in the quality of the data in training and test set.

Statistical analysis will be performed in the training set and the quality of the method will be examined in the test set. Note that the size of the test set is setting equal to $30 \%$ of the data and the rest (i.e, $70 \%$ of data) represents the size of the test set.

3) Mean normalizing the features (average and standard deviations):

Measured data from CCST has various ranges and thus there is a great difference by order of the magnitude in these features. Table II represents both minimum and maximum values for each parameter. As in Table II, the range of the values are diversified (i.e, one parameter can be 100 times bigger than the other). In this case, scaling the features by mean normalization will drive the gradient descent-based algorithm to converge. Therefore, in order to perform gradient descent on the data, it is advised to mean normalize the data as follows:

$$
\frac{X-\mu}{\sigma},
$$

where $X$ denotes the value of the parameter to be normalized, $\mu$ and $\sigma$ represent the average and the standard deviations, respectively. Note that $\mu$ and $\sigma$ are calculated once on the training set and then they applied to normalize the test set.

4) Randomly initializing $\theta$ :

The parameter $\theta$ is the coefficient of the parameters that described in section V-A. Before applying the gradient descent, $\theta$ should be initialized. Herein, is is randomly initialized in the interval $[0,1]$.

5) Optimizing $\theta$ with the gradient descent algorithm: By defining the two parameters (number of iterations and learning rate), the gradient descent algorithm can be applied on the data. One way to verify if the gradient descent algorithm works correctly, it suffies to calculate the cost function and to verify if the value related to the average square of the prediction error as the cost function is decreasing at each iteration.

6) Prediction equation:

After normalizing test set data, using the average and standard deviations of the training set, predicting the variables may be computed as follows:

$$
Y=X \theta
$$

where $X$ denotes the normalized data from the test set and $Y$ is that corresponding to the predicted value.

Of course, for more comprehensive documentary and introductory, the reader can refer to $\mathrm{Ng}$ [7].

\section{Computational Results}

The data are analysed using GNU Octave, version 5.2.0 on a mac OS Catalina with $2.3 \mathrm{GHz}$ Intel Core i5 processor. Parameters tuning of the gradient descent procedure are considered as follows: (i) learning rate $\Lambda$ is set to 0.01 and (ii) the number of iterations is set to 50000 . Note that there are 60 data for each experiments. Thus, the computational time is negligible (less than a minute).

The prediction equations for the two experiments on Lime are provided as follows (cf., Equation 6 and Equation 7):

$\mathrm{Y} 1=0.86-0.05 \mathrm{X} 1+0.05 \mathrm{X} 2-0.20 \mathrm{X} 3+0.01 \mathrm{X} 4-0.26 \mathrm{X} 5+0.23 \mathrm{X} 6-0.01 \mathrm{X} 7+0.29 \mathrm{X} 8$

and

$\mathrm{Y} 2=1.01-0.05 \mathrm{X} 1+0.05 \mathrm{X} 2-0.21 \mathrm{X} 3+-0.07 \mathrm{X} 4-0.87 \mathrm{X} 5+0.90 \mathrm{X} 6-0.01 \mathrm{X} 7-0.27 \mathrm{X} 8$

The same procedure is applied for Cement, where the followings equations (Equation 8 and Equation 9) are used:

$\mathrm{Y} 1=0.92-0.13 \mathrm{X} 1+0.06 \mathrm{X} 2-0.22 \mathrm{X} 3+-0.07 \mathrm{X} 4+0.12 \mathrm{X} 5+-0.19 \mathrm{X} 6+0.13 \mathrm{X} 7+-0.08 \mathrm{X} 8$

and

$\mathrm{Y} 2=1.11-0.05 \mathrm{X} 10.02 \mathrm{X} 2-0.32 \mathrm{X} 3+0.05 \mathrm{X} 4+0.52 \mathrm{X} 5+-0.24 \mathrm{X} 6+0.04 \mathrm{X} 7-0.41 \mathrm{X} 8$

The performance of prediction via gradient descent algorithm to predict variables of CCST is depicted in Figure 1, Figure 2, Figure 3 and Figure 4. From the figures, one can observe what follows:

- First, the gradient descent can provide interesting predictions for the experiments

- Second, the errors of the proposed analysis may be due to the possible human errors in the data set and / or interactions between parameters of the study. We hope that the latter can be faced by applying neural network or logistics regressions instead of gradient descent. 


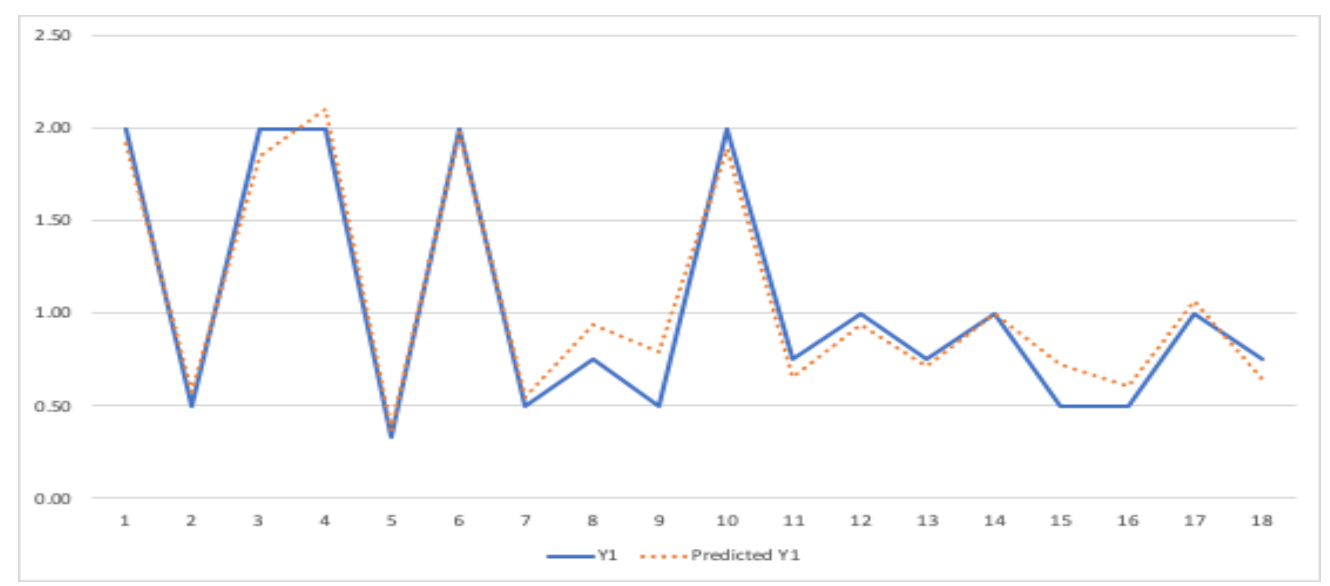

Fig. 1: Prediction of ratio of shive to coating substance $Y 1$ versus ratio of shive to coating substance for the test data for Lime

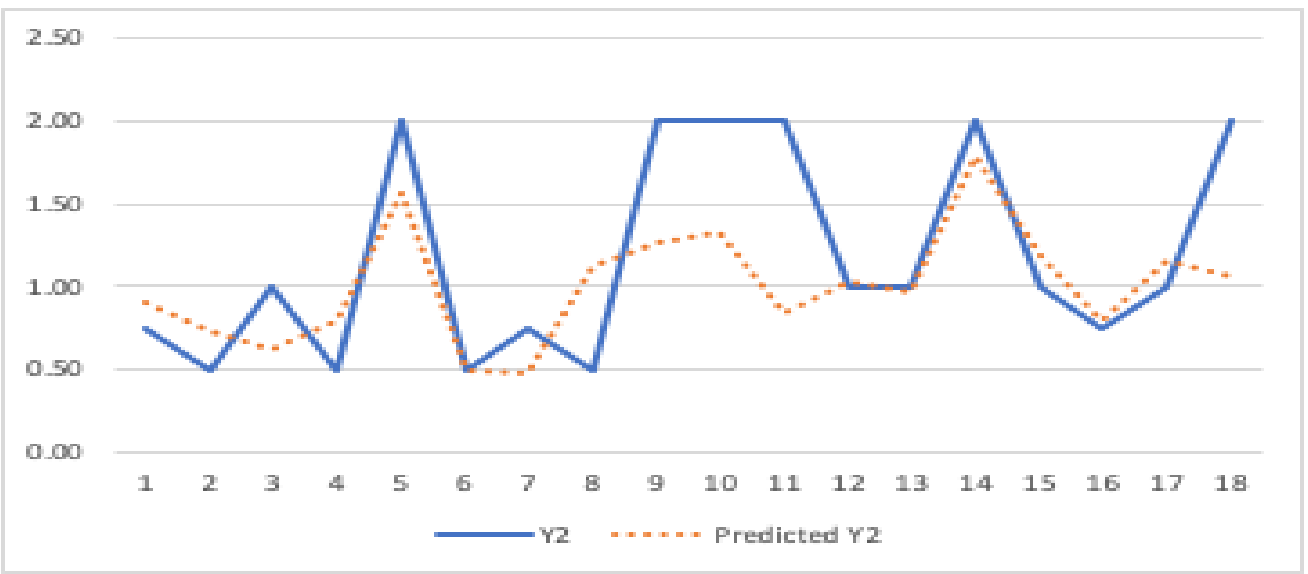

Fig. 2: Prediction of ratio of water to coating substance (Y2) VS ratio of water to coating substance for the test data for Lime

- Third, the average errors reaching the predictions related to the ratio of shive to coating substance and water to coating substance are equal to -0.02 and 0.17 for Lime and, equal to 0.00 and 0.10 for Cement accordingly.

- Fourth and last, the learning rate is set to 0.01 and the number of iterations is set to 60000. As the number of training set is less than 100 , the runtime is not significant (less than a minute). Finally, the established equations are parameterized using descriptions in Table I.

\section{CONCLUSION}

In this study we proposed mathematical equations to simulate the Compressive Cement Strength Test referred as CCST. Such a problem is the most important test in quality control for the industries. In practice, CCST takes 28 days and it is expensive to provide the final results. Therefore, this paper proposed an approach that is able to predict the parameters of the experiment. In order to establish efficient values for the parameters, a gradient descent-based algorithm has been proposed, where linear equations were considered. For future research, several directions of research can be considered. Indeed, first, neural networks can be adapted to predict some parameters and so, in some cases they can be applied for providing tight estimations. Second, the hybridization between neuronal networks and operational research technics can be envisaged for achieving better predictions of the parameters.

\section{REFERENCES}

[1] Snell, L. M., Van Roekel, J.,\& Wallace, N. D. (1989). Predicting early concrete strength. Concrete International, 11(12), 43-47.

[2] de Siqueira Tango, C. E. (1998). An extrapolation method for compressive strength prediction of hydraulic cement products. Cement and concrete research, 28(7), 969-983.

[3] Baykasoğlu, A., Dereli, T., \& Tanış, S. (2004). Prediction of cement strength using soft computing techniques. Cement and concrete research, 34(11), 2083-2090.

[4] Baykasoglu, A., Oztas, A., \& Ozbay, E. (2009). Prediction and multiobjective optimization of high-strength concrete parameters via soft computing approaches. Expert Systems with Applications, 36(3), 61456155.

[5] Ruder, S. (2016). An overview of gradient descent optimization algorithms. arXiv preprint arXiv:1609.04747.

[6] Bengio, Y., Boulanger-Lewandowski, N., \& Pascanu, R. (2013, May). Advances in optimizing recurrent networks. In 2013 IEEE International Conference on Acoustics, Speech and Signal Processing (pp. 86248628). IEEE.

[7] $\mathrm{Ng}$, A. (2018). Machine Learning. Stanford University. línea]. Available: https://www. coursera. org/learn/machine-learning.[Último acceso: 14 apr 2017]. 
TABLE II: Range of parameters

\begin{tabular}{llllllllll} 
Coating substance $(\mathrm{CS})$ & Range & $\mathrm{X} 1(\%)$ & $\mathrm{X} 2(\%)$ & $\mathrm{X} 3(\mathrm{~kg} / \mathrm{m} 3)$ & $\mathrm{X} 4(\mathrm{Mpa})$ & $\mathrm{X} 5(\mathrm{MPa})$ & $\mathrm{X} 6(\mathrm{~kg} / \mathrm{m} 3)$ & $\mathrm{X} 7(\mathrm{~mm} / \mathrm{m})$ & $\mathrm{X} 8(\mathrm{~mm} / \mathrm{m})$ \\
\hline Lime & Min & 36.43 & 47.93 & 129.70 & 0.14 & 0.63 & 583.63 & 2.40 & 1.31 \\
Lime & Max & 77.66 & 136.40 & 298.50 & 2.57 & 9.56 & 1047.93 & 8.17 & 8.65 \\
Cement & $\min$ & 38.23 & 46.81 & 130.60 & 0.16 & 0.31 & 556.09 & 0.00 & 3.14 \\
Cement & $\max$ & 78.18 & 132.54 & 340.75 & 3.28 & 7.41 & 1099.49 & 7.66 & 6.76 \\
\hline
\end{tabular}

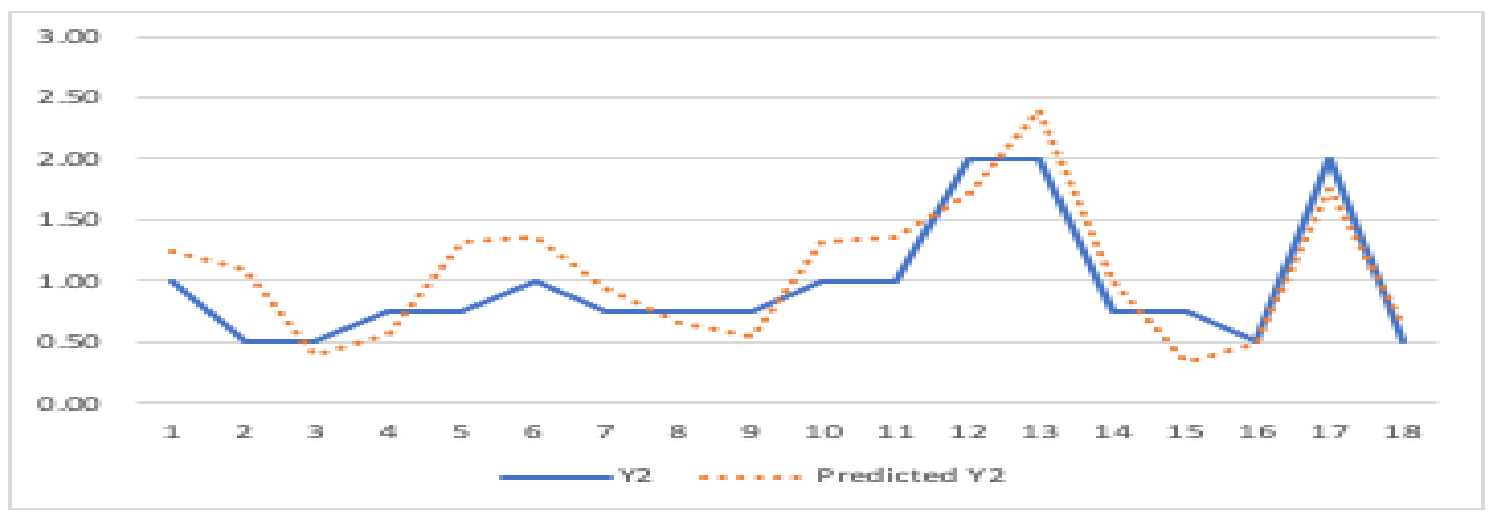

Fig. 3: Prediction of ratio of water to coating substance (Y2) VS ratio of water to coating substance for the test data for Cement

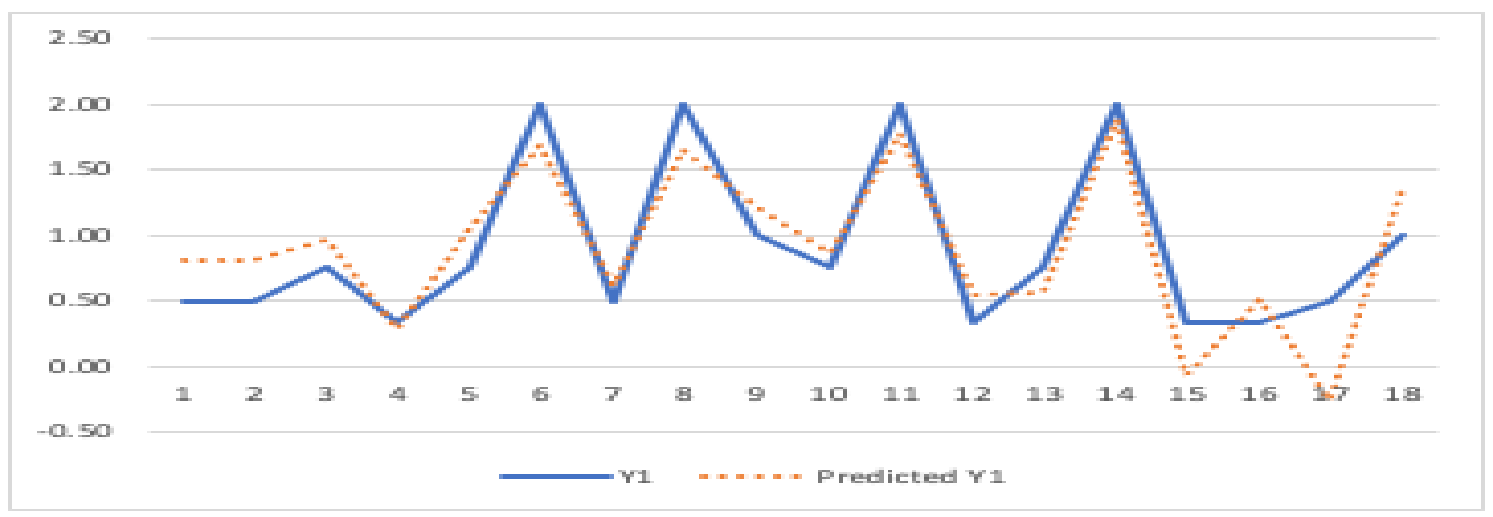

Fig. 4: Prediction of ratio of water to coating substance (Y1) VS ratio of water to coating substance for the test data for Cement 
TABLE III: Average data for each experiment

\begin{tabular}{|c|c|c|c|c|c|c|c|c|c|c|}
\hline Coating substance (CS) & $\mathrm{Y} 1$ (S/CS) & $\mathrm{Y} 2(\mathrm{~W} / \mathrm{CS})$ & $\mathrm{X} 1(\%)$ & $\mathrm{X} 2(\%)$ & $\mathrm{X} 3(\mathrm{~kg} / \mathrm{m} 3)$ & X4 (Mpa) & X5 (MPa) & $\mathrm{X} 6(\mathrm{~kg} / \mathrm{m} 3)$ & $\mathrm{X} 7(\mathrm{~mm} / \mathrm{m})$ & $\mathrm{X} 8(\mathrm{~mm} / \mathrm{m})$ \\
\hline Lime & 0.33 & 0.50 & 66.47 & 256.75 & 71.95 & 1.06 & 6.30 & 819.60 & 4.57 & 2.70 \\
\hline Lime & 0.33 & 0.75 & 60.76 & 261.10 & 84.20 & 1.86 & 8.89 & 1025.34 & 3.15 & 2.24 \\
\hline Lime & 0.33 & 1.00 & 67.97 & 295.13 & 68.72 & 1.79 & 4.88 & 911.35 & 4.81 & 1.95 \\
\hline Lime & 0.33 & 2.00 & 66.39 & 290.80 & 72.12 & 0.28 & 0.67 & 705.04 & 4.50 & 1.49 \\
\hline Lime & 0.50 & 0.50 & 64.46 & 217.43 & 76.25 & 0.55 & 1.77 & 643.55 & 4.57 & 2.99 \\
\hline Lime & 0.50 & 0.75 & 65.80 & 220.68 & 73.38 & 0.83 & 2.79 & 685.20 & 4.06 & 3.02 \\
\hline Lime & 0.50 & 1.00 & 65.59 & 218.68 & 73.84 & 1.53 & 6.39 & 914.18 & 3.25 & 2.85 \\
\hline Lime & 0.50 & 2.00 & 65.98 & 218.68 & 72.99 & 0.83 & 2.05 & 734.88 & 6.11 & 2.51 \\
\hline Lime & 0.67 & 0.50 & 61.14 & 211.98 & 83.38 & 1.01 & 2.23 & 696.17 & 5.64 & 3.35 \\
\hline Lime & 0.67 & 0.75 & 55.93 & 194.02 & 94.56 & 1.01 & 3.04 & 702.38 & 4.65 & 2.76 \\
\hline Lime & 0.67 & 1.00 & 66.40 & 208.57 & 72.08 & 1.72 & 3.64 & 779.18 & 3.89 & 2.71 \\
\hline Lime & 0.67 & 2.00 & 61.57 & 196.97 & 82.46 & 2.41 & 5.00 & 836.20 & 3.17 & 2.28 \\
\hline Lime & 1.00 & 0.50 & 55.57 & 184.07 & 95.32 & 0.59 & 1.46 & 651.26 & 6.29 & 4.68 \\
\hline Lime & 1.00 & 0.75 & 58.73 & 160.58 & 88.55 & 0.55 & 1.76 & 676.64 & 5.29 & 4.09 \\
\hline Lime & 1.00 & 1.00 & 57.80 & 170.38 & 90.54 & 0.79 & 2.33 & 686.95 & 5.03 & 3.65 \\
\hline Lime & 1.00 & 2.00 & 58.27 & 173.57 & 89.54 & 0.87 & 2.60 & 779.21 & 4.57 & 2.80 \\
\hline Lime & 2.00 & 0.50 & 46.76 & 140.00 & 114.22 & 0.19 & 0.83 & 602.45 & 6.68 & 8.22 \\
\hline Lime & 2.00 & 0.75 & 49.55 & 134.87 & 108.25 & 0.20 & 0.85 & 625.43 & 6.32 & 6.90 \\
\hline Lime & 2.00 & 1.00 & 48.82 & 130.93 & 109.81 & 0.22 & 0.95 & 591.77 & 6.28 & 7.53 \\
\hline Lime & 2.00 & 2.00 & 43.83 & 132.43 & 120.53 & 0.28 & 1.06 & 693.85 & 5.85 & 6.22 \\
\hline Cement & 0.33 & 0.50 & 71.48 & 318.27 & 61.19 & 0.55 & 1.29 & 810.66 & 6.09 & 3.76 \\
\hline Cement & 0.33 & 0.75 & 70.43 & 322.43 & 63.44 & 0.90 & 1.97 & 907.19 & 5.01 & 3.55 \\
\hline Cement & 0.33 & 1.00 & 71.34 & 328.15 & 61.48 & 1.10 & 2.98 & 933.54 & 5.01 & 3.26 \\
\hline Cement & 0.33 & 2.00 & 66.44 & 300.75 & 72.01 & 1.58 & 6.34 & 978.46 & 4.58 & 3.32 \\
\hline Cement & 0.50 & 0.50 & 64.47 & 233.48 & 76.22 & 0.67 & 1.59 & 761.58 & 5.04 & 3.86 \\
\hline Cement & 0.50 & 0.75 & 69.20 & 240.23 & 66.08 & 0.67 & 1.74 & 822.90 & 4.72 & 3.59 \\
\hline Cement & 0.50 & 1.00 & 65.88 & 234.35 & 73.21 & 1.33 & 2.21 & 840.21 & 5.21 & 3.43 \\
\hline Cement & 0.50 & 2.00 & 61.89 & 255.72 & 81.77 & 2.34 & 6.47 & 1076.02 & 2.72 & 3.51 \\
\hline Cement & 0.67 & 0.50 & 60.09 & 201.40 & 85.63 & 0.73 & 1.50 & 737.36 & 5.70 & 5.87 \\
\hline Cement & 0.67 & 0.75 & 57.43 & 197.65 & 91.33 & 1.10 & 2.75 & 748.96 & 5.42 & 5.19 \\
\hline Cement & 0.67 & 1.00 & 62.59 & 203.33 & 80.26 & 1.20 & 2.97 & 732.15 & 4.17 & 4.25 \\
\hline Cement & 0.67 & 2.00 & 61.54 & 198.97 & 82.52 & 2.45 & 6.53 & 916.17 & 3.87 & 4.12 \\
\hline Cement & 1.00 & 0.50 & 54.69 & 164.27 & 97.22 & 0.37 & 0.85 & 635.59 & 6.57 & 6.05 \\
\hline Cement & 1.00 & 0.75 & 58.84 & 165.83 & 88.31 & 0.49 & 1.20 & 614.77 & 6.41 & 6.00 \\
\hline Cement & 1.00 & 1.00 & 57.44 & 163.78 & 91.32 & 0.54 & 1.33 & 685.74 & 5.47 & 4.83 \\
\hline Cement & 1.00 & 2.00 & 57.62 & 158.47 & 90.93 & 1.22 & 2.55 & 748.84 & 5.08 & 3.98 \\
\hline Cement & 2.00 & 0.50 & 45.54 & 140.27 & 116.85 & 0.20 & 0.40 & 605.68 & 7.45 & 6.14 \\
\hline Cement & 2.00 & 0.75 & 40.35 & 136.27 & 127.99 & 0.26 & 0.50 & 596.42 & 6.95 & 6.21 \\
\hline Cement & 2.00 & 1.00 & 42.39 & 132.63 & 123.60 & 0.25 & 0.52 & 591.47 & 6.09 & 4.77 \\
\hline Cement & 2.00 & 2.00 & 46.68 & 133.28 & 114.40 & 0.37 & 0.83 & 576.58 & 5.65 & 4.26 \\
\hline
\end{tabular}




\section{An Optimization on Quadrature Formulas and Numerical Solutions of Ordinary Differential Equations}

\author{
Venelin Todorov \\ Bulgarian Academy of Sciences \\ Institute of Mathematics and Informatics \\ Sofia 1113, Bulgaria \\ Bulgarian Academy of Sciences
}

Institute of Information and Communication Technologies

Sofia 1113, Bulgaria

Email: vtodorov@math.bas.bg,venelin@parallel.bas.bg

Radan Miryanov

University of Economics

Department of Statistics and Applied Mathematics

77 Knyaz Boris I Blvd.,

Varna 9002, Bulgaria

Email: miryanov@ue-varna.bg

Stefka Fidanova

Bulgarian Academy of Sciences

Institute of Information and Communication Technologies

ul. G. Bonchev 25A, 1113 Sofia, Bulgaria

Email: stefka@parallel.bas.bg

\author{
Yuri Dimitrov \\ Department of Mathematics and Physics \\ University of Forestry \\ Sofia 1756, Bulgaria \\ Bulgarian Academy of Sciences \\ Institute of Mathematics and Informatics \\ Sofia 1113, Bulgaria \\ Email: yuri.dimitrov@ltu.bg \\ Ivan Dimov \\ Bulgarian Academy of Sciences
}

Institute of Information and Communication Technologies

ul. G. Bonchev 25A, Sofia 1113, Bulgaria

Email: ivdimov@bas.bg

Stoyan Poryazov

Bulgarian Academy of Sciences

Institute of Mathematics and Informatics

ul. G. Bonchev 8, 1113 Sofia, Bulgaria

Email: stoyan@math.bas.bg

\begin{abstract}
This paper is a continuation of the discussion on optimization of the quadrature formulas and their applications in paper [2]. Second-order numerical solutions of Volterra integral equations are constructed using the quadrature formulas obtained in [2]. The numerical results presented in the paper confirm the effectiveness of the methods for numerical solution of ordinary differential equations.
\end{abstract}

\section{INTRODUCTION}

I $\mathrm{N}$ PAPER [2] we study the quadrature formulas which have generating functions $G_{1}(x)=\pi \sec (\pi \sqrt{x} / 2) / 4$ and $G_{2}(x)=\pi \tan (\pi \sqrt{x} / 2) /(4 \sqrt{x})$. We construct the secondorder quadrature formulas

$\frac{h}{2}\left(y_{0}+\sum_{k=1}^{N-1} \bar{E}_{k} y_{N-k}+\frac{\pi-1}{2} y_{N}\right)=\int_{a}^{b} y(x) d x+O\left(h^{2}\right)$,

Venelin Todorov is supported by the National Scientific Program "Information and Communication Technologies for a Single Digital Market in Science, Education and Security (ICT in SES)", contract No DO1-205/23.11.2018, financed by the Ministry of Education and Science in Bulgaria and by the Bulgarian National Science Fund under Project DN 12/5-2017 "Efficien Stochastic Methods and Algorithms for Large-Scale Problems". Yuri Dimitrov is supported by the Bulgarian National Science Fund under Young Scientist Project KP-06-M32/2 - 17.12.2019 "Advanced Stochastic and Deterministic Approaches for Large-Scale Problems of Computational Mathematics".
$\frac{h}{2}\left(y_{0}+\sum_{k=1}^{N-1} \bar{B}_{k} y_{N-k}+\frac{\pi^{2}-6}{4} y_{N}\right)=\int_{a}^{b} y(x) d x+O\left(h^{2}\right)$, where $h=(b-a) / N$ and $x_{k}=a+k h, y_{k}=y\left(x_{k}\right)$ for $k=0,1, \cdots, N$ and $\bar{E}_{k}$ and $\bar{B}_{k}$ are the coefficients of the Maclaurin series of the generating functions

$$
\bar{E}_{k}=\frac{\left|E_{2 k}\right|}{(2 k) !}\left(\frac{\pi}{2}\right)^{2 k+1}, \bar{B}_{k}=\frac{\left(4^{k+1}-1\right) \pi^{2 k+2}\left|B_{2 k+2}\right|}{(2 k+2) !},
$$

where $E_{k}$ and $B_{k}$ are the Euler and Bernoulli numbers. The coefficients of the right endpoint expansion formulas are equal to the coefficients of the Maclaurin series of the functions $H_{i}(x)=G_{i}\left(e^{-x}\right)$ for $i=1,2$. In [2] we construct third-order and fourth-order quadrature formulas as linear combinations of the trapezoidal rule

$$
\frac{h}{2}\left(y_{0}+2 \sum_{k=1}^{N-1} y_{N-k}+y_{N}\right)=\int_{a}^{b} y(x) d x+O\left(h^{2}\right),
$$

the two quadrature formulas above and their modifications. The method for construction of quadrature formulas by first specifying the generating function is an effective method for construction of approximations of the fractional derivatives and integrals (see [1], [3], [4], [5]). The method is applicable 
for construction of approximations of the definite integral and the integer order derivatives of a function as well. In [7] we construct approximations of the first derivative which have exponential and logarithmic generating functions. In the paper we give a proof for the convergence of the approximations and applications of the approximations for numerical solution of ordinary and partial differential equations. The method used in the paper can be extended for construction of approximations of the second derivative which are suitable for deriving approximations of the fractional derivatives. Other methods for numerical solution of integral equations use Gaussian quadratures on non-uniform nets and Monte Carlo methods for numerical integration (see. [8], [9], [10]). Let

$$
\frac{h}{2} \sum_{i=0}^{N} w_{i} y_{N-i}=\int_{a}^{b} y(t) d t+O\left(h^{2}\right)
$$

be a second-order quadrature. Consider a Voltera integral equation of the second kind which has the following form

$$
y(x)+\int_{0}^{x} K(x-t) y(t) d t=F(x) .
$$

The Nyström method (see [6]) for computing the numerical solution of equation (3) uses the approximations of the values of the definite integral in equation (3) with quadrature formula (*) on all intervals $\left[0, x_{n}\right]$,

$$
y_{n}+\frac{h}{2} \sum_{i=0}^{n} w_{i} K_{i} y_{n-i}=F_{n}+O\left(h^{2}\right) .
$$

The numerical solution $\left\{u_{n}\right\}_{n=0}^{N}$ of integral equation (3), where $u_{n}$ is an approximation of the value of the solution $y_{n}$, is computed recursively with

$$
u_{n}=\frac{1}{2+w_{0} K_{0} h}\left(2 F_{n}-h \sum_{i=1}^{n} w_{i} K_{i} y_{n-i}\right)
$$

and has an initial condition $u_{0}=y_{0}=F(0)$. The computation of numerical solution $\mathrm{NS}(*)$ involves $O\left(N^{2}\right)$ operations. Denote by (3.1) and (3.2) the equations obtained from equation (3) with a kernel function $K(s)=3+2 s$ and right hand side $F_{1}(x)=x^{3}\left(10\left(4 x^{2}+30 x+40\right) \ln x-18 x^{2}-75 x\right) / 400$ and

$$
\begin{aligned}
F_{2}(x)=\arctan x-x & +(3 / 2+x) \ln \left(1+x^{2}\right) \\
& +\left(1+3 x+x^{2}\right) \operatorname{arccot} x
\end{aligned}
$$

respectively. Equations (3.1) and (3.2) have the solutions $y(x)=x^{3} \ln x$ and $y(x)=\operatorname{arccot} x$. In table $\mathrm{I}$ we give the experimental results for the error and the order of the numerical solution NS(2), which uses the trapezoidal rule (2), of equations (3.1)-left and (3.2)-right on the interval $[0,1]$. The rest of the paper is organized as follows. In section two we construct the numerical solution of first order ODEs by transforming them to integral equations in the form (3) and we also construct the numerical solutions which use the corresponding shifted quadrature formulas. In section three of the paper we apply the method for numerical solution of second order ordinary differential equations (ODEs) by converting them to Voltera integral equations
TABLE I

\begin{tabular}{|l|cc|cc|}
\hline \multicolumn{1}{|c|}{$h$} & Error & Order & Error & Order \\
\hline 0.005 & $4.545 \times 10^{-6}$ & 2.000 & $2.802 \times 10^{-6}$ & 2.000 \\
0.0025 & $1.136 \times 10^{-6}$ & 2.000 & $7.006 \times 10^{-7}$ & 2.000 \\
0.00125 & $2.841 \times 10^{-7}$ & 2.000 & $1.751 \times 10^{-7}$ & 2.000 \\
\hline
\end{tabular}

\section{NUMERICAL SOLUTION OF FIRST ORDER ODES}

In this section we construct the numerical solution of first order ODEs by first transforming them to integral equations which are solved with Nyström method. In [2] we obtain the second order approximations

$$
\begin{aligned}
& \frac{h}{2} \sum_{k=0}^{N} \bar{E}_{k} y_{N-k}=\int_{a}^{b} y(x) d x+O\left(h^{2}\right), \\
& \frac{h}{2} \sum_{k=0}^{N} \bar{B}_{k} y_{N-k}=\int_{a}^{b} y(x) d x+O\left(h^{2}\right),
\end{aligned}
$$

where $\bar{E}_{0}=(\pi-1) / 2, \bar{B}_{0}=\left(\pi^{2}-6\right) / 4$ and the rest of the weights are defined with (1). The two approximations (4) and (5) require that the integrand function satisfies $y(a)=0$. We use the method (see [3], [7]) for extending the approximations to the class of all differentiable functions by changing the values of the last weights. By applying approximation (4) to the function $y(x)-y(a)$ we obtain

$$
\begin{aligned}
& \frac{h}{2} \sum_{k=0}^{N-1} \bar{E}_{k}\left(y_{N-k}-y_{0}\right)=\int_{a}^{b}(y(x)-y(a)) d x+O\left(h^{2}\right), \\
& \frac{h}{2}\left(\sum_{k=0}^{N-1} \bar{E}_{k} y_{N-k}+2 N y_{0}-y_{0} \sum_{k=0}^{N-1} \bar{E}_{k}\right)=\int_{a}^{b} y(x) d x+O\left(h^{2}\right) .
\end{aligned}
$$

Define $\bar{E}_{N}=2 N-\sum_{k=0}^{N-1} \bar{E}_{k}$. Therefore approximation (4) has a second-order accuracy for all differentiable functions. Similarly, when $\bar{B}_{N}=2 N-\sum_{k=0}^{N-1} \bar{B}_{k}$ approximation (5) holds for all differentiable functions in $[a, b]$. Now we apply approximations (4) and (5) for numerical solution of ODEs. Consider the first order linear ODE

$$
y^{\prime}+a y=f(x), y(0)=y_{0} .
$$

By applying integration on both sides of equation (6) we get

$$
\begin{aligned}
\int_{0}^{x} y^{\prime}(t) d t+a \int_{0}^{x} y(t) d t & =\int_{0}^{x} f(t) d t \\
y(x)+a \int_{0}^{x} y(t) d t & =F(x)
\end{aligned}
$$

where $F(x)=y_{0}+\int_{0}^{x} y(t) d t$. Equation (7) is equivalent to (6) and it is a Voltera integral equation of the second kind with a kernel $K(s)=a$. Denote by (7.1) the equation which corresponds to (7) when $a=2$ and $F_{1}(x)=x^{3}(4(2+$ $x) \ln x-x) / 8$. Equation (7.1) has the solution $y(x)=x^{3} \ln x$ and it is equivalent to equation (6) with a right hand side $f_{1}(x)=x^{2}(1+(3+2 x) \ln x)$. Denote by (7.2) the integral equation which corresponds to equation (7) with $a=3$, 
$F_{2}(x)=(1+3 x) \operatorname{arccot} x+3 \ln \left(1+x^{2}\right) / 2$ and initial condition $y(0)=y_{0}=F_{0}(0)=\pi / 2$. Equation (7.2) has the solution $y(x)=\arccos x$ and it is equivalent to equation (6) with a right hand side $f_{2}(x)=3 \operatorname{arccot} x-1 /\left(1+x^{2}\right)$. Equations (7.1) and (7.2) are integral equations in the form (3) and can be solved numerically with methods NS(4) and NS(5), which use quadrature formulas (4) and (5). In table II we give the experimental results for the error and the order of the numerical solution NS(4) of equations (7.2) and (7.3).

TABLE II

\begin{tabular}{|l|cc|cc|}
\hline \multicolumn{1}{|c|}{$h$} & Error & Order & Error & Order \\
\hline 0.005 & $7.202 \times 10^{-6}$ & 1.995 & $2.918 \times 10^{-6}$ & 1.969 \\
0.0025 & $1.804 \times 10^{-6}$ & 1.997 & $7.412 \times 10^{-7}$ & 1.977 \\
0.00125 & $4.513 \times 10^{-7}$ & 1.999 & $1.870 \times 10^{-7}$ & 1.987 \\
\hline
\end{tabular}

The results for the error and order of the numerical solution NS(5) of equations (7.2) and (7.3) are given in table III.

TABLE III

\begin{tabular}{|l|cc|cc|}
\hline \multicolumn{1}{|c|}{$h$} & Error & Order & Error & Order \\
\hline 0.005 & $3.823 \times 10^{-6}$ & 2.004 & $1.351 \times 10^{-6}$ & 1.996 \\
0.0025 & $9.544 \times 10^{-7}$ & 2.002 & $3.385 \times 10^{-7}$ & 1.997 \\
0.00125 & $2.384 \times 10^{-7}$ & 2.001 & $8.476 \times 10^{-8}$ & 1.998 \\
\hline
\end{tabular}

Now we construct the shifted quadratures which correspond to formulas (4) and (5). From [2]

$$
\begin{aligned}
& \frac{h}{2} \sum_{k=0}^{N} \bar{E}_{k} y_{N-k}=\int_{a}^{b} y(x) d x+\frac{1}{4} f(b) h+O\left(h^{2}\right), \\
& \frac{h}{2} \sum_{k=0}^{N} \bar{B}_{k} y_{N-k}=\int_{a}^{b} y(x) d x+\frac{3}{4} f(b) h+O\left(h^{2}\right),
\end{aligned}
$$

where the weights $\bar{E}_{k}$ and $\bar{B}_{k}$ are defined with (1) for all indices $k=0,1, \cdots, n$. From the mean value theorem we have the second order approximation

$$
\int_{a}^{b+c h} y(x) d x=\int_{a}^{b} y(x) d x+c f(b) h+O\left(h^{2}\right) .
$$

From (8) and (10)

$$
\frac{h}{2} \sum_{k=0}^{N} \bar{E}_{k} y_{N-k}=\int_{a}^{b+h / 4} y(x) d x+O\left(h^{2}\right) .
$$

Shifted quadature (11) has a requirement that the integrand function satisfies the condition $y(a)=0$. By applying (11) to the function $y(x)-y(a)$ we find

$\frac{h}{2} \sum_{k=0}^{N-1} \bar{E}_{k}\left(y_{N-k}-y_{0}\right)=\int_{a}^{b+h / 4}(y(x)-y(a)) d x+O\left(h^{2}\right)$,

Shifted quadrature (11) has a second order accuracy for all differentiable functions when the weight $\bar{E}_{N}$ is defined as

$$
\bar{E}_{N}=\frac{2}{h}\left(b-a+\frac{h}{4}\right)-\sum_{k=0}^{N-1} \bar{E}_{k}=2 N+\frac{1}{2}-\sum_{k=0}^{N-1} \bar{E}_{k}
$$

Similarly from (9) and (10) we obtain

$$
\frac{h}{2} \sum_{k=0}^{N} \bar{B}_{k} y_{N-k}=\int_{a}^{b+3 h / 4} y(x) d x+O\left(h^{2}\right) .
$$

Shifted quadrature (12) has a second-order accuracy when

$$
\bar{B}_{N}=2 N+\frac{3}{2}-\sum_{k=0}^{N-1} \bar{B}_{k}
$$

and the weights $\bar{B}_{0}, \bar{B}_{1}, \cdots, \bar{B}_{N-1}$ are defined with (1). The first weights $\bar{E}_{0}=\pi / 2$ and $\bar{B}_{0}=\pi^{2} / 4$. Now we construct the numerical solution of integral equation (7) which uses shifted quadrature (11). By approximating the definite integral in (7) at the point $x_{n+1 / 4}$ with (11) we obtain

$$
y_{n+1 / 4}+\frac{1}{2} a h \sum_{k=0}^{n-1} \bar{E}_{k} y_{n-k}=F_{n+1 / 4}+O\left(h^{2}\right) .
$$

Let $\left\{u_{n}\right\}_{n=0}^{N}$ be the numerical solution of (7). From the second order approximation

$$
y_{n+1 / 4}=\frac{5 y_{n}-y_{n-1}}{4}+O\left(h^{2}\right)
$$

we obtain the recursive formula for the numerical solution

$$
u_{n}=\frac{1}{5+\pi a h}\left(4 F_{n+1 / 4}+u_{n-1}-2 a h \sum_{k=1}^{n-1} \bar{E}_{k} y_{n-k}\right) \text {, }
$$

with an initial condition $u_{0}=F_{0}$. In table IV we give the experimental results for the error and the order of the numerical solution NS1 of equations (7.2)-left and (7.3)-right.

TABLE IV

\begin{tabular}{|l|cc|cc|}
\hline \multicolumn{1}{|c|}{$h$} & Error & Order & Error & Order \\
\hline 0.005 & $5.638 \times 10^{-6}$ & 2.000 & $1.323 \times 10^{-6}$ & 1.996 \\
0.0025 & $1.409 \times 10^{-6}$ & 2.000 & $3.314 \times 10^{-7}$ & 1.998 \\
0.00125 & $3.522 \times 10^{-7}$ & 2.000 & $8.291 \times 10^{-8}$ & 1.989 \\
\hline
\end{tabular}

The numerical solution of integral equation (7) which uses shifted quadrature (12) is obtained from the approximation of the definite integral in (7) at the point $x_{n+3 / 4}$ with (12).

$$
y_{n+3 / 4}+\frac{1}{2} a h \sum_{k=0}^{n-1} \bar{B}_{k} y_{n-k}=F_{n+3 / 4}+O\left(h^{2}\right) .
$$

From

$$
y_{n+3 / 4}=\frac{7 y_{n}-3 y_{n-1}}{4}+O\left(h^{2}\right)
$$

we obtain the recursive formula of the numerical solution

$$
u_{n}=\frac{2}{14+a \pi^{2} h}\left(4 F_{n+3 / 4}+3 u_{n-1}-2 a h \sum_{k=1}^{n-1} \bar{E}_{k} y_{n-k}\right) \text {. }
$$

The experimental results for the error and the order of the numerical solution NS2 of equations (7.2) and (7.3) are given in table V. 
TABLE V

\begin{tabular}{|l|cc|cc|}
\hline \multicolumn{1}{|c|}{$h$} & Error & Order & Error & Order \\
\hline 0.005 & $6.249 \times 10^{-5}$ & 1.990 & $1.352 \times 10^{-5}$ & 1.900 \\
0.0025 & $1.567 \times 10^{-5}$ & 1.995 & $3.532 \times 10^{-6}$ & 1.936 \\
0.00125 & $3.924 \times 10^{-6}$ & 1.997 & $9.054 \times 10^{-7}$ & 1.964 \\
\hline
\end{tabular}

\section{NUMERICAL SOLUTION OF SECOND ORDER ODES}

We apply the method from section 2 for computing the numerical solution of the second order ODE

$$
y^{\prime \prime}+3 y^{\prime}+2 y=f(x), y(0)=y_{0}, y^{\prime}(0)=y_{0}^{\prime} .
$$

Equation (13) is transformed to an integral equation in the form (3) by applying successive integration to both sides

$$
\begin{gathered}
\int_{0}^{x} y^{\prime \prime}(u) d u+3 \int_{0}^{x} y^{\prime}(u) d u+2 \int_{0}^{x} y(u) d u=\int_{0}^{x} f(u) d u, \\
y^{\prime}(x)+3 y(x)+2 \int_{0}^{x} y(u) d u=3 y_{0}+y_{0}^{\prime}+\int_{0}^{x} f(u) d u .
\end{gathered}
$$

Integrate again both sides

$$
y(x)+3 \int_{0}^{x} y(t) d t+2 \int_{0}^{x} \int_{0}^{t} f(u) d u d t=F(x),
$$

where $F(x)=y_{0}+\left(3 y_{0}+y_{0}^{\prime}\right) x+\int_{0}^{x} \int_{0}^{t} f(u) d u d t$. By changing the order of integration of the double integral we get

$\int_{0}^{x} \int_{0}^{t} f(u) d u d t=\int_{0}^{x} \int_{u}^{x} f(u) d t d u=\int_{0}^{x}(x-u) f(u) d t d u$.

Equation (14) is transformed to

$$
y(x)+\int_{0}^{x}(3+2 x-2 t) y(t) d t=F(x) .
$$

Equation (15) is a Volterra integral equation of the second kind with a kernel $K(s)=3+2 s$. In section 1 we compute the numerical solution $\mathrm{NS}(2)$ of equations (3.1) and (3.2), which are also integral equations in the form (15). In table VI we give the results for the maximal error and order of numerical solution NS(4), which uses quadrature formula (4), of equations (3.1)left and (3.2)-right and step sizes $h=0.005,0.0025,0.00125$.

TABLE VI

\begin{tabular}{|l|cc|cc|}
\hline \multicolumn{1}{|c|}{$h$} & Error & Order & Error & Order \\
\hline 0.005 & $6.730 \times 10^{-6}$ & 1.997 & $5.885 \times 10^{-6}$ & 1.947 \\
0.0025 & $1.684 \times 10^{-6}$ & 1.998 & $1.499 \times 10^{-7}$ & 1.972 \\
0.00125 & $4.213 \times 10^{-7}$ & 1.999 & $3.805 \times 10^{-7}$ & 1.978 \\
\hline
\end{tabular}

The results for the error and the order of the numerical solution $\mathrm{NS}(5)$ of equations (3.1) and (3.2) are given in table VII.

TABLE VII

\begin{tabular}{|l|cc|cc|}
\hline \multicolumn{1}{|c|}{$h$} & Error & Order & Error & Order \\
\hline 0.005 & $3.563 \times 10^{-6}$ & 2.002 & $2.795 \times 10^{-6}$ & 2.008 \\
0.0025 & $8.901 \times 10^{-7}$ & 2.001 & $6.969 \times 10^{-7}$ & 2.004 \\
0.00125 & $2.224 \times 10^{-7}$ & 2.000 & $1.740 \times 10^{-7}$ & 2.001 \\
\hline
\end{tabular}

\section{CONCLUSION}

In the paper we construct second-order numerical solutions of ordinary differential equations by converting them to integral equations and applying the quadrature formulas from [2] . Numerical solutions NS(4) and NS(5) involve additional multiplications compared to the standard method NS(2) and have a longer computational time. One advantage of the methods discussed in the paper is that the linear ODEs are equivalent to Volterra integral equations in the form (3) and they can be solved numerically with $\mathrm{NS}(*)$ which uses an appropriate quadrature. All methods discussed in the paper involve $O\left(N^{2}\right)$ computations and have comparable performance. From the experiments presented in the paper and the results of additional experiments we can conclude that the numerical solutions discussed in the paper have a stable and efficient performance. In future work we will prove the convergence of the numerical solutions constructed in the paper and we will apply the methods for numerical solution of other classes of ordinary differential equations.

\section{REFERENCES}

[1] Y. Dimitrov, "Approximations for the Caputo derivative (I)," Journal of Fractional Calculus and Applications, vol. 9 no. 1, 2018, pp. 35-63, http://fcag-egypt.com/Journals/JFCA/.

[2] Y. Dimitrov, R. Miryanov and V. Todorov, "Quadrature formulas and Taylor series of secant and tangent", Economics and computer science, vol. 4, 2017, pp. 23-40.

[3] Y. Dimitrov, R. Miryanov and V. Todorov, "Asymptotic expansions and approximations of the Caputo derivative", Computational and Applied Mathematics, vol. 37 no. 4, 2018, pp. 5476-5499, https://doi.org/10.1007/s40314-018-0641-3.

[4] H. Ding and C. Li, "High-order numerical algorithms for Riesz derivatives via constructing new generating functions", Journal of Scientific Computing, vol. 71 no. 3, 2017, pp. 759-784, https://doi.org/10.1007/s10915-016-0317-3.

[5] G. H. Gao, Z. Z. Sun and H. W. Zhang, "A new fractional numerical differentiation formula to approximate the Caputo fractional derivative and its applications", Journal of Computational Physics, vol. 259, 2014, pp. 33-50, https://doi.org/10.1016/j.jcp.2013.11.017.

[6] E. J. Nyström, "Über die praktische Auflösung von Integralgleichungen mit Anwendungen auf Randwertaufgaben", Acta Mathematica, vol. 54 no. 1, 1930, pp. 185-204, https://doi.org/10.1007/BF02547521.

[7] V. Todorov, Y. Dimitrov and I. Dimov, "Second order shifted approximations for the first derivative", Studies in Computational Intelligence Proc. Inter. Conf. HPC 2019, Borovetz, Bulgaria, 2019, in press.

[8] R. Farnoosh and M. Ebrahimi, "Monte Carlo method for solving Fredholm integral equations of the second kind", Applied Mathematics and Computation, vol. 195, 2008, pp. 309-315, https://doi.org/10.1016/j.amc.2007.04.097.

[9] S. Kim, "Solving singular integral equations using Gaussian quadrature and overdetermined system", Computers \& Mathematics with Applications, vol. 35 no. 10,1998 , pp. 63-71, https://doi.org/10.1016/S08981221(98)00073-X.

[10] Y. Zaizai and H. Zhimin, "Using the Monte Carlo method to solve integral equations using a modified control variate", $A p$ plied Mathematics and Computation, vol. 242, 2014, pp. 764-777, https://doi.org/10.1016/j.amc.2014.06.079. 


\section{An Optimal Monte Carlo algorithm for a Class of Multidimensional Integrals}

\section{Ivan Dimov} \\ Bulgarian Academy of Sciences
}

Venelin Todorov

Bulgarian Academy of Sciences

Institute of Mathematics and Informatics

ul. G. Bonchev 8, 1113 Sofia, Bulgaria

Bulgarian Academy of Sciences

Institute of Information and Communication Technologies

ul. G. Bonchev 25A, 1113 Sofia, Bulgaria

Email: vtodorov@math.bas.bg,venelin@ parallel.bas.bg

Institute of Information and Communication Technologies

ul. G. Bonchev 25A, 1113 Sofia, Bulgaria

Email: ivdimov@bas.bg

Stoyan Poryazov

Bulgarian Academy of Sciences

Institute of Mathematics and Informatics

ul. G. Bonchev 8, 1113 Sofia, Bulgaria

Email: stoyan@math.bas.bg

\author{
Stoyan Apostolov \\ Sofia University \\ Faculty of Mathematics and Informatics \\ 1764 Sofia, Bulgria \\ Email: stoyanrapostolov@gmail.com
}

\author{
Stefka Fidanova \\ Bulgarian Academy of Sciences \\ Institute of Information and Communication Technologies \\ ul. G. Bonchev 25A, 1113 Sofia, Bulgaria \\ Email: stefka@parallel.bas.bg
}

\author{
Yuri Dimitrov \\ Department of Mathematics and Physics \\ University of Forestry \\ Sofia 1756, Bulgaria \\ Bulgarian Academy of Sciences \\ Institute of Mathematics and Informatics \\ Sofia 1113, Bulgaria \\ Email: yuri.dimitrov@ltu.bg
}

\begin{abstract}
An optimal stochastic approach for multidimensional integrals of smooth functions. This is the first time this optimal stochastic approach has been compared with other stochastic approaches for mid and high dimensions. The purpose of the present study is to compare the optimal algorithm with the lattice rules based on the generalized Fibonacci numbers of the corresponding dimension and to discuss the advantages and disadvantages of each method.
\end{abstract}

\section{INTRODUCTION}

The Monte Carlo method has proven to be very useful tool for numerical analysis, particularly when the number of dimension ranging from medium to large. Monte Carlo simulation and quasi-Monte Carlo methods are the prevailing methods used to solve multi-dimensional problems in different areas. Both methods do not suffer from the ,curse of the dimensionality" [2] This is the first time to study optimal

Stoyan Apostolov and Yuri Dmitrov are supported by the Bulgarian National Science Fund under Young Scientists Project KP-06-M32/2 17.12.2019 "Advanced Stochastic and Deterministic Approaches for LargeScale Problems of Computational Mathematics". Venelin Todorov is supported by the National Scientific Program "Information and Communication Technologies for a Single Digital Market in Science, Education and Security (ICT in SES)", contract No DO1-205/23.11.2018, financed by the Ministry of Education and Science in Bulgaria and by the National Scientific Program for post doctoral and young scientists of Ministry of Education and Science 2020-2021. stochastic algorithms for computing multidimensional integrals of smooth functions. The optimal approach has been established by Atanasov and Dimov in [1] but there aren't any comparison with other stochastic approaches up to now.

\section{Description of the Optimal Monte Carlo ALGORITHM}

Most Monte Carlo methods improve achieves better convergence using the idea of dividing the area of integration [2]. In the case where, in addition to the idea of dividing the area $\Omega=U^{s}$ (but without the recursive element), the information on the smoothness of the subintegral function is used, an increase in the order of convergence is achieved. Our first known results in this area with probability density $p(\mathbf{x})=1$, and splitting the area into equal parts in all directions are obtained by Dupach ([4]).

Theorem 1: (Dupach, [4]). Let $g(\mathbf{x})$ and all its first-order partial derivatives $\frac{\partial g}{\partial x_{k}}$ are continuous in $\Omega$ and bounded, i.e. for all $1 \leq k \leq s:\left|\frac{\partial g}{\partial x_{k}}\right| \leq L$, and there exist constants $c_{1}, c_{2}>0$, for which are fulfilled the following conditions

$$
p_{j} \leq \frac{c_{1}}{N}, \quad j=1, \ldots, N, \quad d_{j} \leq \frac{c_{2}}{N^{1 / s}},
$$


where $d_{j}$ is the diameter of the subdomain $\Omega_{j}$, i.e. $d_{j}=$ $\sup \left|\mathbf{x}_{1}-\mathbf{x}_{2}\right|$. Then for the estimation of the variance $\mathbf{x}_{1}, \mathbf{x}_{2} \in \Omega_{j}$

we obtain $\bar{\theta}_{N}^{*}$ in the case of $M=N \quad N_{j}=1, j=1, \ldots, M$ and:

$$
\mathbf{D} \bar{\theta}_{N}^{*} \leq c^{2} L^{2} N^{-1-2 / s}, \quad \text { where } \quad c=s c_{1} c_{2} .
$$

Using Chebyshev's inequality [?], [?], for the error $\bar{\theta}_{N}^{*}-I[g]$ we obtain

$$
P\left\{\left|\bar{\theta}_{N}^{*}-I[g]\right|<\frac{c L}{\varepsilon} N^{-1 / 2-1 / s}\right\} \geq 1-\varepsilon^{2},
$$

where $\varepsilon$ is a small enough positive number.

Given the equation (1) it can be concluded that the probable error $\bar{\theta}_{N}^{*}-I[g]$ decreases in order $N^{-1 / 2-1 / s}$, i.e. faster than $N^{-1 / 2}$. Obviously for large dimensions $s$ the acceleration of the convergence order is relatively insignificant.

The same result for the order of convergence can be achieved in less favorable conditions, namely, only that the corresponding function is continuous. The proof is made by Dimov and Tonev ([3]), and the statement is formulated in the following theorem:

Theorem 2: (Dimov, Tonev; [3]). Let $g$ is continuous in $\Omega \subset \mathbf{R}^{s}$ and there exist constants $c_{1}, c_{2}, c_{3}$, such that $p_{j} \leq$ $c_{1} / N, d_{j} \leq c_{2} N^{-1 / s}$ and $S_{j}\left(\cdot, c_{3}\right) \subset \Omega_{j}, j=1, \ldots, N$ (i.e. $M=N$ and $\left.N_{j}=1\right)$, where $S_{j}\left(\cdot, c_{3}\right)$ is sphere with radius $c_{3}$. Then for the probable error $r_{N}$ is fulfilled [2]:

$$
r_{N} \leq 4 \sqrt{2} \frac{c_{1} c_{2}}{c_{3}} \tau\left(g ; d_{\max }\right)_{L_{q}} N^{-1 / 2-1 / s},
$$

where $d_{\max }=\max _{j} d_{j} \tau(g ; \delta)_{L_{q}}$ is the average modulus of continuity, that is,

$$
\begin{gathered}
\tau(g ; \delta)_{L_{q}}=\|\omega(g, \cdot ; \delta)\|_{L_{q}}=\left(\int_{\Omega}(\omega(g, \mathbf{x} ; \delta))^{q} \mathrm{~d} \mathbf{x}\right)^{1 / q} \\
1 \leq q \leq \infty, \delta \in\left[0, d_{\max }\right] \text { and }
\end{gathered}
$$

$\omega(g, \mathbf{x} ; \delta)=\sup \left\{\left|\Delta_{h} g(\mathrm{t})\right|: \mathrm{t}, \mathrm{t}+h \in[\mathbf{x}-\delta / 2, x+\delta / 2] \cap \Omega\right\}$,

where $\Delta_{h}$ is the difference (forward) of the function $g$ in the point $t$ from first order, which in this case plays a role similar to the derivative but in a discrete sense.

Similar to the one obtained by Dupach, a higher order variance estimate can also be derived for a class of functions whose higher order derivatives are constrained by adding additional conditions for the choice of random points in the subdomains. Rayna Georgieva proves the following theorem [5]:

Theorem 3: Let we have domain $\Omega \in \mathbf{R}^{s}$ and the division of the domain $\Omega=\sum_{j=1}^{M} \Omega_{j}$, where every subdomain $\Omega_{j}$ is centrally symmetric with center $p_{j}$. Let $\xi^{(j)}$ is a random $s$ dimensional point, uniformly distributed in $\Omega_{j}, \xi^{(j)^{\prime}}$ is symmetric (about the center $p_{j}$ ) with $\xi^{(j)}$, i.e. $\xi^{(j)}+\xi^{(j)^{\prime}}=2 p_{j}$. If the first and second partial derivatives of a function $g(\mathbf{x})$ are continuous in $\Omega$ and for all $k, l=1, \ldots, s$ is fulfilled that

$$
\frac{\partial^{2} g}{\partial x_{k} \partial x_{l}} \leq L \quad d_{j}=\sup _{x_{1}, x_{2} \in \Omega_{j}}\left|x_{1}-x_{2}\right| \leq \frac{c_{1}}{N^{1 / s}},
$$

then $\mathbf{D} \bar{\theta}_{N}^{* *}=c_{2} L^{2} N^{-1-4 / s}$, where $\bar{\theta}_{N}^{* *}=\sum_{j=1}^{N} \frac{V_{j}}{2}\left[g\left(\xi^{(j)}\right)+\right.$ $\left.g\left(\xi^{(j)^{\prime}}\right)\right], V_{j}$ is the volume of the subdomain $\Omega_{j} \quad c=$ $\left(0.5 s^{2} c_{1}^{s+2}\right)^{2}$

There are various Monte Carlo approaches for numerical integration whose convergence order is $\mathcal{O}\left(N^{-\frac{1}{2}-\frac{k}{s}}\right)$. For $k=1$ and $k=2$ these methods can be constructed relatively easily [2], following Dupach's ideas for $k=1$, described above (see the Theorem 1). But the situation changes when $k \geq 3$. Using the method of controlling the variance on interpolation polynomials, Atanasov and Dimov [1] formulate conditions for constructing a method with optimal order of convergence for $s$-dimensional functions from the class $W^{k}$. The optimal method requires $m * n^{s}$ points and is briefly described below [1].

For $n, s, k \geq 1$ we define integration formula of type Monte Carlo, depends on an integer parameter $m \geq 1$ and $\left(\begin{array}{c}s+k-1 \\ s\end{array}\right)$ points in $[0,1]^{s}$ by the following way. Points $x^{(r)}$ are exactly a number of $\left({ }^{s+k-1}{ }_{s}\right)$ and they must fulfil the condition, that if for one polynom $P(x)$, so that for the degree of the polynom $\operatorname{deg} P \leq k$ is fulfilled $P\left(x^{(r)}\right)=0$, then $P \equiv 0$. If $N=n^{s}$ for $n \geq 1$ we divide $s$ dimensional hypercube $[0,1]^{s}$ into $n^{s}$ endless undercubes $K_{j}$, i.e. $[0,1]^{s}=c_{i=1}^{n^{s}} K_{j}$ and $K_{j}=\prod_{i=1}^{s}\left[a_{i}^{j}, b_{i}^{j}\right)$, where $b_{i}^{j}-a_{i}^{j}=\frac{1}{n}$ for every $i=1, \ldots, s$. For every cube $K_{j}$ we calculate the coordinates of $\left(\begin{array}{c}s+k-1 \\ s\end{array}\right)$ points $y^{(r)}$, defined by $y_{i}^{(r)}=a_{i}^{r}+\frac{1}{n} x_{i}(r)$. We assume that we choose $m$ random points $\xi_{i}(j, s)=\left(\xi_{1}(j, p), \ldots, \xi_{s}(j, p)\right)$ from every cube $K_{j}$, such that all the points $\xi_{i}(j, p)$ are independent uniformly distributed random points, we calculate all $f\left(y^{(r)}\right)$ and $f\left(\xi_{i}(j, p)\right)$, and we calculate the Lagrange polynom of the function $f$ in the point $z$ using information about the functional values in the points $y^{(r)}$. We denote the polynom by $L_{k}(f, z)$. For every polynom $P$ of max degree $k-1$ we have that $L_{k}(f, z) \equiv z$. Now we approximate

$\int_{K_{j}} f(x) d x \approx \frac{1}{m n^{s}} \sum_{s=1}^{m}\left[(\xi(j, p))-L_{k}(f, \xi(j, p))\right]+\int_{K_{j}} L_{k}(f, x) d x$.

After that we sum for every $j=1, \ldots, N$ and obtain:

$I(f) \approx \frac{1}{m n^{s}} \sum_{j=1}^{N} \sum_{s=1}^{m}\left[(\xi(j, p))-L_{k}(f, \xi(j, p))\right]+\sum_{j=1}^{N} \int_{K_{j}} L_{k}(f, x) d x$.

$\int_{K_{j}} f(x) d x \approx \frac{1}{m n^{s}} \sum_{s=1}^{m}\left[(\xi(j, p))-L_{k}(f, \xi(j, p))\right]+\int_{K_{j}} L_{k}(f, x) d x$.

After that we sum for every $j=1, \ldots, N$ and obtain:

$I(f) \approx \frac{1}{m n^{s}} \sum_{j=1}^{N} \sum_{s=1}^{m}\left[(\xi(j, p))-L_{k}(f, \xi(j, p))\right]+\sum_{j=1}^{N} \int_{K_{j}} L_{k}(f, x) d x$.

\section{NUMERICAL EXAMPLES}

We will test the optimal method on multidimensional integrals of smooth functions of different dimensions. We use the following notations: $\mathrm{A}==$ Adaptive stochastic approach, $\mathrm{L}=$ Latin Hypercube sampling, $\mathrm{S}=$ Sobol quasi-random sequence, $\mathrm{F}=$ Fibonacci lattice rule, $\mathrm{O}=$ optimal approach. For more information about the description of the different stochastic approaches, see [2], [6]. We must emphasise 
Table I

RELATIVE ERROR FOR THE 3 DIMENSIONAL INTEGRAL DIMENSIONAL INTEGRAL

\begin{tabular}{|c|c|c|c|c|c|c|c|c|c|c|}
\hline \hline $\mathrm{N}$ & $\mathrm{A}$ & $\mathrm{t}$ & $\mathrm{F}$ & $\mathrm{t}$ & $\mathrm{S}$ & $\mathrm{t}$ & $\mathrm{L}$ & $\mathrm{t}$ & $\mathrm{O}$ & $\mathrm{t}$ \\
\hline $10^{3}$ & $4.82 \mathrm{e}-3$ & 0.17 & $1.21 \mathrm{e}-3$ & 0.006 & $4.87 \mathrm{e}-4$ & 0.47 & $6.14 \mathrm{e}-3$ & 0.004 & $3.12 \mathrm{e}-5$ & 0.81 \\
\hline $10^{4}$ & $1.07 \mathrm{e}-3$ & 1.44 & $5.04 \mathrm{e}-4$ & 0.07 & $1.56 \mathrm{e}-4$ & 1.88 & $6.56 \mathrm{e}-4$ & 0.06 & $2.05 \mathrm{e}-6$ & 4.13 \\
\hline $10^{5}$ & $1.52 \mathrm{e}-4$ & 10.9 & $5.34 \mathrm{e}-6$ & 0.66 & $2.51 \mathrm{e}-5$ & 15.6 & $1.34 \mathrm{e}-4$ & 0.51 & $4.58 \mathrm{e}-7$ & 31.62 \\
\hline $10^{6}$ & $5.11 \mathrm{e}-5$ & 131 & $7.85 \mathrm{e}-7$ & 7.02 & $7.43 \mathrm{e}-6$ & 105.80 & $6.84 \mathrm{e}-5$ & 5.22 & $6.72 \mathrm{e}-8$ & 155 \\
\hline $10^{7}$ & $2.34 \mathrm{e}-5$ & 1094 & $8.89 \mathrm{e}-8$ & 79.7 & $1.58 \mathrm{e}-6$ & 934 & $1.73 \mathrm{e}-5$ & 17 & $5.34 \mathrm{e}-9$ & 1053 \\
\hline \hline
\end{tabular}

Table II

RELATIVE ERROR FOR THE 3 DIMENSIONAL INTEGRAL

\begin{tabular}{|c|c|c|c|c|c|c|}
\hline \hline time(s) & C & A & F & S & L & O \\
\hline 1 & $1.05 \mathrm{e}-3$ & $7.96 \mathrm{e}-3$ & $2.34 \mathrm{e}-6$ & $2.93 \mathrm{e}-4$ & $5.11 \mathrm{e}-4$ & $1.21 \mathrm{e}-5$ \\
\hline 5 & $6.84 \mathrm{e}-4$ & $8.14 \mathrm{e}-4$ & $8.47 \mathrm{e}-7$ & $8.01 \mathrm{e}-5$ & $7.32 \mathrm{e}-5$ & $1.12 \mathrm{e}-6$ \\
\hline 10 & $4.79 \mathrm{e}-4$ & $1.82 \mathrm{e}-4$ & $4.89 \mathrm{e}-7$ & $4.71 \mathrm{e}-5$ & $4.32 \mathrm{e}-5$ & $7.21 \mathrm{e}-7$ \\
\hline 100 & $1.57 \mathrm{e}-4$ & $7.04 \mathrm{e}-5$ & $6.53 \mathrm{e}-9$ & $7.68 \mathrm{e}-6$ & $5.32 \mathrm{e}-6$ & $8.61 \mathrm{e}-8$ \\
\hline \hline
\end{tabular}

on the fact this is the first time a comprehensive experimental study with this optimal approach and other stochastic methods under consideration has been done.

Example 1. $\mathrm{s}=3$.

$$
\int_{[0,1]^{3}} \exp \left(x_{1} x_{2} x_{3}\right) \approx 1.14649907 .
$$

Example 2. $s=4$.

$$
\int_{[0,1]^{4}} x_{1} x_{2}^{2} e^{x_{1} x_{2}} \sin \left(x_{3}\right) \cos \left(x_{4}\right) \approx 0.1089748630 .
$$

Example 3. s= 5.

$$
\int_{[0,1]^{5}} \exp \left(-100 x_{1} x_{2} x_{3}\right)\left(\sin \left(x_{4}\right)+\cos \left(x_{5}\right)\right) \approx 0.1854297367
$$

Example 4. $\mathrm{s}=7$.

$$
\int_{[0,1]^{7}} e^{1-\sum_{i=1}^{3} \sin \left(\frac{\pi}{2} \cdot x_{i}\right)} \cdot \arcsin \left(\sin (1)+\frac{\sum_{j=1}^{7} x_{j}}{200}\right) \approx 0.75151101 .
$$

Example 5. $\mathrm{s}=15$.

$$
\int_{[0,1]^{15}}\left(\sum_{i=1}^{10} x_{i}^{2}\right)\left(x_{11}-x_{12}^{2}-x_{13}^{3}-x_{14}^{4}-x_{15}^{5}\right)^{2} \approx 1.96440666 .
$$

Example 6. $s=25$.

$$
\int_{[0,1]^{2} 5} \frac{4 x_{1} x_{3}^{2} e^{2 x_{1} x_{3}}}{\left(1+x_{2}+x_{4}\right)^{2}} e^{x_{5}+\cdots+x_{20}} x_{21} \ldots x_{25} \approx 108.808 .
$$

Example 7. $\mathrm{s}=30$.

$$
\int_{[0,1]^{3} 0} \frac{4 x_{1} x_{3}^{2} e^{2 x_{1} x_{3}}}{\left(1+x_{2}+x_{4}\right)^{2}} e^{x_{5}+\cdots+x_{20}} x_{21} \ldots x_{30} \approx 3.244540 .
$$

For smooth integrands without computational peculiarities Fibonacci lattice algorithm gives better results for lower dimensions,
Table III

RELATIVE ERROR FOR THE 4 DIMENSIONAL INTEGRAL

\begin{tabular}{|c|c|c|c|c|c|c|c|c|c|c|}
\hline $\mathrm{N}$ & $\mathrm{A}$ & $\mathrm{t}$ & $\mathrm{F}$ & $\mathrm{t}$ & $\mathrm{S}$ & $\mathrm{t}$ & $\mathrm{L}$ & $\mathrm{t}$ & $\mathrm{O}$ & $\mathrm{t}$ \\
\hline $10^{4}$ & $3.11 \mathrm{e}-4$ & 1.97 & $2.61 \mathrm{e}-5$ & 0.07 & $2.61 \mathrm{e}-5$ & 2.14 & $5.29 \mathrm{e}-4$ & 0.07 & $1.52 \mathrm{e}-5$ & 4.81 \\
\hline $10^{5}$ & $2.44 \mathrm{e}-5$ & 20.1 & $5.62 \mathrm{e}-6$ & 0.99 & $5.93 \mathrm{e}-6$ & 17.6 & $3.56 \mathrm{e}-4$ & 0.60 & $7.96 \mathrm{e}-6$ & 45.1 \\
\hline $10^{6}$ & $1.13 \mathrm{e}-5$ & 210 & $5.86 \mathrm{e}-7$ & 5.22 & $1.51 \mathrm{e}-6$ & 193 & $4.36 \mathrm{e}-5$ & 4.97 & $2.31 \mathrm{e}-7$ & 352.6 \\
\hline $10^{7}$ & $8.11 \mathrm{e}-6$ & 2035 & $8.38 \mathrm{e}-9$ & 58 & $8.30 \mathrm{e}-7$ & 1121 & $8.12 \mathrm{e}-6$ & 47.1 & $8.16 \mathrm{e}-9$ & 2651 \\
\hline
\end{tabular}

Table IV

RELATIVE ERROR FOR THE 4 DIMENSIONAL INTEGRAL

\begin{tabular}{|c|c|c|c|c|c|c|}
\hline \hline time(s) & C & A & F & S & L & O \\
\hline 0.1 & $3.48 \mathrm{e}-3$ & $3.59 \mathrm{e}-4$ & $1.44 \mathrm{e}-5$ & $4.07 \mathrm{e}-4$ & $4.18 \mathrm{e}-4$ & $4.22 \mathrm{e}-5$ \\
\hline 1 & $1.48 \mathrm{e}-3$ & $2.85 \mathrm{e}-4$ & $5.62 \mathrm{e}-6$ & $3.54 \mathrm{e}-5$ & $3.32 \mathrm{e}-4$ & $2.31 \mathrm{e}-5$ \\
\hline 5 & $6.62 \mathrm{e}-4$ & $9.18 \mathrm{e}-5$ & $5.38 \mathrm{e}-7$ & $5.26 \mathrm{e}-5$ & $4.23 \mathrm{e}-5$ & $1.12 \mathrm{e}-5$ \\
\hline 10 & $2.52 \mathrm{e}-4$ & $1.36 \mathrm{e}-5$ & $3.77 \mathrm{e}-7$ & $6.50 \mathrm{e}-6$ & $3.48 \mathrm{e}-5$ & $7.53 \mathrm{e}-6$ \\
\hline 20 & $1.58 \mathrm{e}-4$ & $2.08 \mathrm{e}-5$ & $2.67 \mathrm{e}-8$ & $4.55 \mathrm{e}-6$ & $2.16 \mathrm{e}-5$ & $6.54 \mathrm{e}-7$ \\
\hline
\end{tabular}

but it requires more random points when the dimensionality increases. The MC algorithm based on Latin hypercube sampling has higher accuracy for this case study with increasing the dimensionality of the integral. The adaptive MC algorithm is slower, but it requires smaller number of random points to achieve better accuracy even for higher dimensions. The efficiency of the optimal $\mathrm{C}$ algorithm is clearly seen for lower dimensions.

As the dimension increases, the computational time of the optimal method decrease due to the increased computational time of the Lagrangian interpolation polynomial. But for very high dimensions 25 and 30 the relative error for a fixed computational time is better than this achieved by the other stochastic methods. From the Tables it is obvious that for small dimensions the FIBO and Optimal method achieves the best accuracy for a fixed preliminary given time - see Tables II,IV,VI. For dimensions up to 10, the FIBO and Optimal methods achieve the smallest relative error for a given number of samples - see Tables I,III,V. The Optimal method is with 1 order better than the FIBO method for a fixxed number of samples, but the advantage of FIBO method is the significantly lower computational time. It is seen that for dimensions 10 and 15, the Optimal method gives relative errors for a fixed number of samples with at least 1

Table V

RELATIVE ERROR FOR THE 5 DIMENSIONAL INTEGRAL

\begin{tabular}{|c|c|c|c|c|c|c|c|c|c|c|}
\hline \hline $\mathrm{N}$ & $\mathrm{A}$ & $\mathrm{t}$ & $\mathrm{F}$ & $\mathrm{t}$ & $\mathrm{S}$ & $\mathrm{t}$ & $\mathrm{L}$ & $\mathrm{t}$ & $\mathrm{O}$ & $\mathrm{t}$ \\
\hline $10^{3}$ & $2.15 \mathrm{e}-3$ & 0.27 & $1.75 \mathrm{e}-4$ & 0.007 & $5.29 \mathrm{e}-4$ & 0.03 & $9.38 \mathrm{e}-3$ & 0.007 & $2.75 \mathrm{e}-5$ & 2.1 \\
\hline $10^{4}$ & $2.01 \mathrm{e}-3$ & 2.43 & $1.28 \mathrm{e}-5$ & 0.06 & $1.43 \mathrm{e}-4$ & 0.3 & $3.44 \mathrm{e}-3$ & 0.07 & $7.22 \mathrm{e}-6$ & 2.3 \\
\hline $10^{5}$ & $6.91 \mathrm{e}-4$ & 22.2 & $9.50 \mathrm{e}-6$ & 0.61 & $2.36 \mathrm{e}-5$ & 2.77 & $2.01 \mathrm{e}-3$ & 0.69 & $2.36 \mathrm{e}-6$ & 6.2 \\
\hline $10^{6}$ & $2.92 \mathrm{e}-4$ & 219.5 & $5.47 \mathrm{e}-7$ & 5.98 & $6.07 \mathrm{e}-6$ & 24.2 & $1.80 \mathrm{e}-4$ & 6.17 & $5.46 \mathrm{e}-7$ & 20.0 \\
\hline $10^{7}$ & $8.21 \mathrm{e}-5$ & 2043 & $8.71 \mathrm{e}-9$ & 58.4 & $2.30 \mathrm{e}-6$ & 245 & $2.46 \mathrm{e}-5$ & 60.5 & $7.01 \mathrm{e}-8$ & 105.1 \\
\hline
\end{tabular}

Table VI

RELATIVE ERROR FOR THE 5 DIMENSIONAL INTEGRAL

\begin{tabular}{|c|c|c|c|c|c|}
\hline \hline time(s) & A & F & S & L & O \\
\hline 0.1 & $3.16 \mathrm{e}-3$ & $3.48 \mathrm{e}-3$ & $1.09 \mathrm{e}-5$ & $1.34 \mathrm{e}-4$ & $3.21 \mathrm{e}-3$ \\
\hline 1 & $1.08 \mathrm{e}-3$ & $2.08 \mathrm{e}-3$ & $5.58 \mathrm{e}-6$ & $7.21 \mathrm{e}-5$ & $8.54 \mathrm{e}-4$ \\
\hline 5 & $8.79 \mathrm{e}-4$ & $8.20 \mathrm{e}-4$ & $8.71 \mathrm{e}-7$ & $1.54 \mathrm{e}-5$ & $3.25 \mathrm{e}-4$ \\
\hline 10 & $5.85 \mathrm{e}-4$ & $7.51 \mathrm{e}-4$ & $4.15 \mathrm{e}-7$ & $9.32 \mathrm{e}-6$ & $8.65 \mathrm{e}-5$ \\
\hline 20 & $3.99 \mathrm{e}-4$ & $6.95 \mathrm{e}-4$ & $8.37 \mathrm{e}-8$ & $7.39 \mathrm{e}-6$ & $5.02 \mathrm{e}-5$ \\
\hline
\end{tabular}


Table VII

RELATIVE ERROR FOR THE 7 DIMENSIONAL INTEGRAL

\begin{tabular}{|c|c|c|c|c|c|c|c|c|c|c|}
\hline \hline $\mathrm{N}$ & $\mathrm{A}$ & $\mathrm{t}$ & $\mathrm{F}$ & $\mathrm{t}$ & $\mathrm{S}$ & $\mathrm{t}$ & $\mathrm{L}$ & $\mathrm{t}$ & $\mathrm{O}$ & $\mathrm{t}$ \\
\hline $10^{4}$ & $1.07 \mathrm{e}-3$ & 2.07 & $2.19 \mathrm{e}-3$ & 0.11 & $2.27 \mathrm{e}-4$ & 0.76 & $1.79 \mathrm{e}-3$ & 0.13 & $2.13 \mathrm{e}-4$ & 10.2 \\
\hline $10^{5}$ & $7.51 \mathrm{e}-4$ & 19.3 & $6.19 \mathrm{e}-4$ & 0.99 & $1.22 \mathrm{e}-4$ & 7.45 & $2.53 \mathrm{e}-4$ & 1.15 & $4.41 \mathrm{e}-5$ & 40.2 \\
\hline $10^{6}$ & $6.30 \mathrm{e}-5$ & 194 & $1.99 \mathrm{e}-5$ & 9.81 & $4.71 \mathrm{e}-5$ & 72.3 & $8.27 \mathrm{e}-5$ & 10.32 & $1.27 \mathrm{e}-6$ & 167.1 \\
\hline $10^{7}$ & $2.34 \mathrm{e}-5$ & 1861 & $4.89 \mathrm{e}-7$ & 94.2 & $9.45 \mathrm{e}-6$ & 697 & $1.69 \mathrm{e}-5$ & 101.2 & $1.45 \mathrm{e}-7$ & 595.1 \\
\hline \hline
\end{tabular}

Table VIII

RELATIVE ERROR FOR THE 7 DIMENSIONAL INTEGRAL

\begin{tabular}{|c|c|c|c|c|c|}
\hline \hline time(s) & A & F & S & L & O \\
\hline 0.1 & $2.38 \mathrm{e}-2$ & $3.11 \mathrm{e}-2$ & $2.38 \mathrm{e}-3$ & $1.85 \mathrm{e}-3$ & $2.37 \mathrm{e}-3$ \\
\hline 1 & $8.87 \mathrm{e}-3$ & $2.88 \mathrm{e}-3$ & $6.19 \mathrm{e}-4$ & $1.85 \mathrm{e}-4$ & $3.37 \mathrm{e}-4$ \\
\hline 5 & $5.16 \mathrm{e}-3$ & $3.76 \mathrm{e}-3$ & $8.81 \mathrm{e}-5$ & $9.79 \mathrm{e}-5$ & $1.38 \mathrm{e}-4$ \\
\hline 10 & $1.28 \mathrm{e}-3$ & $6.71 \mathrm{e}-4$ & $1.88 \mathrm{e}-5$ & $8.36 \mathrm{e}-5$ & $8.78 \mathrm{e}-5$ \\
\hline 20 & $2.03 \mathrm{e}-3$ & $4.28 \mathrm{e}-4$ & $3.87 \mathrm{e}-6$ & $5.46 \mathrm{e}-5$ & $6.87 \mathrm{e}-5$ \\
\hline
\end{tabular}

Table IX

RELATIVE ERROR FOR THE 15 DIMENSIONAL INTEGRAL

\begin{tabular}{|c|c|c|c|c|c|c|c|c|c|c|}
\hline \hline $\mathrm{N}$ & $\mathrm{A}$ & $\mathrm{t}$ & $\mathrm{F}$ & $\mathrm{t}$ & $\mathrm{S}$ & $\mathrm{t}$ & $\mathrm{L}$ & $\mathrm{t}$ & $\mathrm{O}$ & $\mathrm{t}$ \\
\hline $10^{3}$ & $3.16 \mathrm{e}-3$ & 9.24 & $5.34 \mathrm{e}-2$ & 0.08 & $2.04 \mathrm{e}-3$ & 0.98 & $1.06 \mathrm{e}-2$ & 0.12 & $7.54 \mathrm{e}-3$ & 27.4 \\
\hline $10^{4}$ & $1.49 \mathrm{e}-3$ & 88 & $1.22 \mathrm{e}-3$ & 0.93 & $2.89 \mathrm{e}-4$ & 9.3 & $7.33 \mathrm{e}-3$ & 1.07 & $6.51 \mathrm{e}-4$ & 81.5 \\
\hline $10^{5}$ & $5.76 \mathrm{e}-4$ & 847 & $3.08 \mathrm{e}-4$ & 9.65 & $1.13 \mathrm{e}-4$ & 93.8 & $1.54 \mathrm{e}-3$ & 10.11 & $7.29 \mathrm{e}-5$ & 242.1 \\
\hline $10^{6}$ & $1.29 \mathrm{e}-4$ & 8235 & $1.37 \mathrm{e}-5$ & 96.9 & $1.93 \mathrm{e}-5$ & 935 & $1.14 \mathrm{e}-4$ & 99.6 & $8.29 \mathrm{e}-6$ & 720.2 \\
\hline \hline
\end{tabular}

order better than FIBO and Sobol, see Tables VII and IX, with FIBO

Table X

RELATIVE ERROR FOR THE 15 DIMENSIONAL INTEGRAL

\begin{tabular}{|c|c|c|c|c|c|}
\hline \hline time(s) & $\mathrm{A}$ & $\mathrm{F}$ & $\mathrm{S}$ & $\mathrm{L}$ & $\mathrm{O}$ \\
\hline 1 & $9.96 \mathrm{e}-1$ & $6.30 \mathrm{e}-3$ & $1.10 \mathrm{e}-3$ & $3.64 \mathrm{e}-3$ & $3.51 \mathrm{e}-2$ \\
\hline 5 & $7.72 \mathrm{e}-2$ & $1.68 \mathrm{e}-3$ & $2.45 \mathrm{e}-4$ & $7.32 \mathrm{e}-4$ & $1.23 \mathrm{e}-2$ \\
\hline 10 & $1.33 \mathrm{e}-2$ & $5.89 \mathrm{e}-3$ & $9.48 \mathrm{e}-5$ & $1.94 \mathrm{e}-4$ & $9.63 \mathrm{e}-3$ \\
\hline 20 & $3.03 \mathrm{e}-2$ & $1.66 \mathrm{e}-3$ & $9.87 \mathrm{e}-6$ & $4.05 \mathrm{e}-5$ & $7.51 \mathrm{e}-3$ \\
\hline 100 & $8.11 \mathrm{e}-3$ & $5.04 \mathrm{e}-4$ & $8.17 \mathrm{e}-7$ & $4.03 \mathrm{e}-6$ & $9.51 \mathrm{e}-5$ \\
\hline \hline
\end{tabular}

Table XI

RELATIVE ERROR FOR THE 25 DIMENSIONAL INTEGRAL

\begin{tabular}{|c|c|c|c|c|c|c|}
\hline \hline $\mathrm{N}$ & $\mathrm{O}$ & $\mathrm{t}, \mathrm{s}$ & $\mathrm{S}$ & $\mathrm{t}, \mathrm{s}$ & $\mathrm{L}$ & $\mathrm{t}, \mathrm{s}$ \\
\hline $10^{3}$ & $3.77 \mathrm{e}-3$ & 2.03 & $1.47 \mathrm{e}-1$ & 0.4 & $7.54 \mathrm{e}-1$ & 0.02 \\
\hline $10^{4}$ & $3.18 \mathrm{e}-3$ & 19.52 & $5.68 \mathrm{e}-2$ & 5.64 & $5.39 \mathrm{e}-2$ & 0.15 \\
\hline $10^{5}$ & $5.33 \mathrm{e}-5$ & 181 & $7.21 \mathrm{e}-3$ & 33.4 & $2.11 \mathrm{e}-2$ & 1.07 \\
\hline $10^{6} 3$ & $3.11 \mathrm{e}-5$ & 1234 & $2.89 \mathrm{e}-3$ & 161 & $1.71 \mathrm{e}-4$ & 8.21 \\
\hline \hline
\end{tabular}

Table XII

RELATIVE ERROR FOR THE 25 DIMENSIONAL INTEGRAL

\begin{tabular}{|c|c|c|c|}
\hline \hline time,sec & $\mathrm{O}$ & $\mathrm{S}$ & $\mathrm{L}$ \\
\hline 1 & $7.24 \mathrm{e}-2$ & $9.51 \mathrm{e}-2$ & $2.11 \mathrm{e}-2$ \\
\hline 5 & $8.16 \mathrm{e}-3$ & $5.76 \mathrm{e}-2$ & $1.61 \mathrm{e}-2$ \\
\hline 10 & $5.18 \mathrm{e}-3$ & $2.71 \mathrm{e}-2$ & $9.58 \mathrm{e}-3$ \\
\hline 20 & $3.13 \mathrm{e}-3$ & $8.28 \mathrm{e}-3$ & $7.87 \mathrm{e}-3$ \\
\hline \hline
\end{tabular}

Table XIII

RELATIVE ERROR FOR 30 DIMENSIONAL INTEGRAL

\begin{tabular}{|c|c|c|c|c|c|c|}
\hline \hline $\mathrm{N}$ & $\mathrm{O}$ & $\mathrm{t}, \mathrm{s}$ & $\mathrm{S}$ & $\mathrm{t}, \mathrm{s}$ & $\mathrm{L}$ & $\mathrm{t}, \mathrm{s}$ \\
\hline $10^{3}$ & $2.01 \mathrm{e}-2$ & 5.4 & $1.18 \mathrm{e}-1$ & 0.42 & $8.81 \mathrm{e}-1$ & 0.02 \\
\hline $10^{4}$ & $6.53 \mathrm{e}-3$ & 14.5 & $8.40 \mathrm{e}-2$ & 4.5 & $6.19 \mathrm{e}-2$ & 0.14 \\
\hline $10^{5}$ & $1.35 \mathrm{e}-3$ & 145 & $1.18 \mathrm{e}-2$ & 30.2 & $2.78 \mathrm{e}-2$ & 1.16 \\
\hline $10^{6}$ & $2.11 \mathrm{e}-4$ & 1290 & $9.20 \mathrm{e}-3$ & 168 & $9.86 \mathrm{e}-3$ & 8.61 \\
\hline \hline
\end{tabular}

Table XIV

RELATIVE ERROR FOR 30 DIMENSIONAL INTEGRAL

\begin{tabular}{|c|c|c|c|}
\hline \hline time,sec & $\mathrm{O}$ & $\mathrm{S}$ & $\mathrm{L}$ \\
\hline 1 & $4.38 \mathrm{e}-1$ & $1.01 \mathrm{e}-1$ & $2.38 \mathrm{e}-2$ \\
\hline 5 & $1.16 \mathrm{e}-2$ & $7.76 \mathrm{e}-2$ & $1.81 \mathrm{e}-2$ \\
\hline 10 & $8.11 \mathrm{e}-3$ & $5.71 \mathrm{e}-2$ & $9.48 \mathrm{e}-3$ \\
\hline 20 & $4.63 \mathrm{e}-3$ & $1.28 \mathrm{e}-2$ & $7.87 \mathrm{e}-3$ \\
\hline
\end{tabular}

achieving better accuracy in much less time - see Tables VIII and X. The adaptive algorithm is suitable for higher dimensions because of the small number of samples to achieve the preliminary given relative error. The Adaptive algorithm performs better than FIBO and Sobol - see Tables XI and XIII. Analyzes show that for high 25 and 30 samples, the Optimal MC approach outperforms LHS and Sobol's quasi-Monte Carlo algorithm - see Tables XII and XIV, where the LHS method produce better results than the Sobol QMC approach. Here we do not test Adaptive approach, because of the significant amount of time and FIBO method which gives unreliable relative errors for the high dimensional case.

\section{CONCLUSIONS}

The efficiency of the optimal Monte Carlo algorithm for the calculation of multidimensional integrals has been presented. The optimal approach appears to be an efficient stochastic solution to solve this kind of problem, because we demonstrate here its reliability for calculating integrals with smooth integrands in a relatively small subregion of the original integration domain regardless of the dimension.

\section{REFERENCES}

[1] E. Atanassov and I.T. Dimov, A new optimal monte carlo method for calculating integrals of smooth functions, Journal of Monte Carlo Methods and Applications 5 (1999), no. 2, 149-167, https://doi.org/10.1515/mcma.1999.5.2.149.

[2] Dimov I. (2008) Monte Carlo Methods for Applied Scientists, New Jersey, London, Singapore, World Scientific, 291 p., ISBN-10 981-022329-3.

[3] I.T. Dimov and O. Tonev, Monte carlo algorithms: performance analysis for some computer architectures, J. of Computational and Applied Mathematics 48 (1993), 253-277, https://doi.org/10.1016/03770427(93)90024-6.

[4] V. Dupach, Stochasticke pocetni metody, Cas. pro pest. mat. 81 (1956), no. $1,55-68$.

[5] Georgieva, R.: PhD Thesis: Computational complexity of Monte Carlo algorithms for multidimensional integrals and integral equations, Sofia, 2003

[6] I.M. Sobol, (1989) Quasi-Monte Carlo methods, in: Sendov, Bl., Dimov, I.T. (eds.) International Youth Workshop on Monte Carlo Methods and Parallel Algorithms, World Scientific, Singapore: 75-81. 


\section{A New Optimized Stochastic Approach for Multiple Integrals in Option Pricing}

\author{
Venelin Todorov \\ Bulgarian Academy of Sciences \\ Institute of Mathematics and Informatics \\ ul. G. Bonchev 8, 1113 Sofia, Bulgaria \\ Bulgarian Academy of Sciences \\ Institute of Information and Communication Technologies \\ ul. G. Bonchev 25A, 1113 Sofia, Bulgaria \\ Email: vtodorov@math.bas.bg,venelin@parallel.bas.bg

\section{Stefka Fidanova \\ Bulgarian Academy of Sciences} \\ Institute of Information and Communication Technologies \\ ul. G. Bonchev 25A, 1113 Sofia, Bulgaria \\ Email: stefka@parallel.bas.bg
}

\begin{abstract}
In the present paper we evaluate European style options with an exponential payoff function with an optimized lattice rule based on a new optimal generating vector. A brief introduction of the theory of lattice rule has been given. We compare the performance of the new stochastic approach with a new optimal generating vector for multiple integrals up to 50 dimensions.
\end{abstract}

\section{INTRODUCTION}

Computational finance is one of the most important topic nowadays [4]. Nowadays Monte Carlo (MC) and quasi-Monte Carlo (QMC) methods have become a popular computational device for problems in finance [8]. The field of mathematical finance is becoming more sophisticated and quantitative and the scope of its applications is growing. The QMC methods using special deterministic sequences achieve higher accuracy and computational efficiency compared to the MC methods. Applications of low discrepancy sequences to financial problems are studied in [3]. Options have been widely traded since the creation of the organized exchange in 1973. The famous Black-Scholes model provides explicit closed form solutions for the values of the European style call and put options. Besides the applications in option pricing, Monte Carlo methods are also used in other problems in finance [2] and other branches of science.

Venelin Todorov is supported by the National Scientific Program "Young scientists and Postdoctoral candidates" 2020-2021. Venelin Todorov and Stoayn Poryazov are partially supported by Task 1.2.5 of National Scientific Program "Information and Communication Technologies for a Single Digital Market in Science, Education and Security (ICT in SES)", contract No DO1-205/23.11.2018, financed by the Ministry of Education and Science in Bulgaria. The work is also supported by the Bulgarian National Science Fund under Project DN 12/5-2017 "Efficient Stochastic Methods and Algorithms for Large-Scale Problems".

\author{
Ivan Dimov \\ Bulgarian Academy of Sciences \\ Institute of Information and Communication Technologies \\ ul. G. Bonchev 25A, 1113 Sofia, Bulgaria \\ Email: ivdimov@bas.bg
}

\author{
Stoyan Poryazov \\ Bulgarian Academy of Sciences \\ Institute of Mathematics and Informatics \\ ul. G. Bonchev 8, 1113 Sofia, Bulgaria \\ Bulgarian Academy of Sciences \\ Email: stoyan@math.bas.bg
}

Definition 1: A European call (put) option is a contract which gives its holder a right, but not an obligation, to buy (sell) predefined quantity of underlying asset $S$ at a prescribed time $T$ (expiry date) at a prescribed exercise (strike) price $E$. An American call (put) option is the same as the European call (put), except that the American options can be exercised at any time during their lifetime, not just at expiry.

The definitions of the risk neutrality and the risk-free interest rate $r$ can be found in [8].

\section{DESCRIPTION OF THE OPTION PRICING PROBLEM}

The value of an asset $S$ as a function of time is modeled by the stochastic differential equation

$$
\mathrm{d} S=\mu S \mathrm{~d} t+\sigma S \mathrm{~d} X
$$

where $\sigma$ is the volatility of the asset, characterizing the fluctuations in the price, $\mu$ is the drift rate, which is a measure of the average rate of growth of the asset price and $\mathrm{d} X$ is the increment of a standard Wiener process. The wellknown Black-Scholes (BS) model for European call options is described with the following partial differential equation

$$
\frac{\partial V}{\partial t}+\frac{1}{2} \sigma^{2} S^{2} \frac{\partial^{2} V}{\partial S^{2}}+r S \frac{\partial V}{\partial S}-r V=0
$$

where $r$ is the risk-free interest rate. The value $V(S, t)$ of the call option satisfies the final condition at the time of expiry $V(S, T)=\max (S-E, 0)$, where $E$ is the strike price. The boundary conditions of a Dirichlet type are as follows:

$$
V(0, t)=0, \quad V(S, t) \sim S-E e^{-r(T-t)}, S \rightarrow \infty .
$$

The European put options satisfy the same BS equation (2) with final and boundary conditions

$$
V(S, T)=\max (E-S, 0),
$$




$$
V(0, t)=E e^{-r(T-t)}, \quad V(S, t) \sim 0, S \rightarrow \infty .
$$

The analytical solution of the BS equation for call options is expressed in an explicit closed form as

$$
V(S, t)=C(S, t)=S \mathrm{~N}\left(d_{1}\right)-E e^{-r(T-t)} \mathrm{N}\left(d_{2}\right),
$$

where

$$
\begin{aligned}
& d_{1}=\frac{\ln (S / E)+\left(r+\sigma^{2} / 2\right)(T-t)}{\sigma \sqrt{T-t}}, \\
& d_{2}=\frac{\ln (S / E)+\left(r-\sigma^{2} / 2\right)(T-t)}{\sigma \sqrt{T-t}},
\end{aligned}
$$

and $\mathrm{N}(z)$ is the cumulative distribution function of the standard normal distribution. Similarly, the European put options price is computed with the formula

$$
V(S, t)=P(S, t)=E e^{-r(T-t)} \mathrm{N}\left(-d_{2}\right)-S \mathrm{~N}\left(-d_{1}\right) .
$$

MC and QMC methods are suitable when the solution is expressed as the expectation of a random variable, which is the case of the risk-neutral evaluation formula for the European options [8]:

$$
V(S, t)=E\left(e^{-r(T-t)} h(S(T)) \mid S(t)=S, \mu=r\right),
$$

where $E$ is the expectation operator and $h(S)$ is the payoff function. The payoff function of the European option satisfies

$$
h(S)=\max (S-E, 0)
$$

for the call option and

$$
h(S)=\max (E-S, 0)
$$

for the put option.

Let's deal with a European call option whose payoff depends on $k>1$ assets with prices $S_{i}, i=1, \ldots, k$. Each asset is modeled by a random walk (1)

$$
\mathrm{d} S_{i}=\mu_{i} S_{i} \mathrm{~d} t+\sigma_{i} S_{i} \mathrm{~d} X_{i}
$$

where $\sigma_{i}$ is the annualized standard deviation for the $i$-th asset and $\mathrm{d} X_{i}$ is an increment of a Brownian motion. The Brownian motion is a special type Markov stochastic process, which has the property $\mathrm{d} X \sim \mathrm{N}(0, \sqrt{d t})$, where $\mathrm{N}(\mu, \sigma)$ is the normal distribution with mean $\mu$ and variance $\sigma^{2}$.

Now suppose that at expiry time $T$, the payoff is given by $h\left(S_{1}^{\prime}, \ldots, S_{k}^{\prime}\right)$, where $S^{\prime}$ denotes the value of the $i$-th asset at expiry. Assuming risk neutrality, the value of the option satisfies

$$
\begin{gathered}
V=e^{-r(T-t)}(2 \pi(T-t))^{-k / 2}(\operatorname{det} \Sigma)^{-1 / 2}\left(\sigma_{1} \ldots \sigma_{k}\right)^{-1} \\
\int_{0}^{\infty} \ldots \int_{0}^{\infty} \frac{h\left(S_{1}^{\prime}, \ldots, S_{k}^{\prime}\right)}{S_{1}^{\prime} \ldots S_{k}^{\prime}} \\
\exp \left(-0.5 \alpha^{\top} \Sigma^{-1} \alpha\right) \mathrm{d} S_{1}^{\prime} \ldots \mathrm{d} S_{k}^{\prime}
\end{gathered}
$$

where

$$
\alpha_{i}=\left(\sigma_{i}(T-t)^{1 / 2}\right)^{-1}\left(\ln \left(S_{i}^{\prime} / S_{i}\right)-\left(r-\sigma_{i}^{2} / 2\right)(T-t)\right),
$$

$r$ is the risk-free interest rate and $\Sigma$ is the covariance matrix, whose entry with index $(i, j)$ is the covariance $\mathrm{d} X_{i}$ and $\mathrm{d} X_{j}$ of the $k$ assets. We will consider the special case when the payoff function is the exponent function which is important for some application in finance, see [8].

\section{DESCRIPTION OF THE NEW OPTIMIZED LATTICE RULE}

Consider the quadrature formula

$$
I_{N}(f)=\frac{1}{N} \sum_{i=0}^{N-1} f\left(x_{i}\right)
$$

where $P_{N}=\left\{x_{0}, x_{1}, \ldots, x_{N-1}\right\}, x_{i} \in[0,1)^{s}$ are the integration nodes of the formula.

The integration nodes, of the lattice rules proposed by Korobov [6], are defined by the following formula:

$$
x_{k}=\left(\left\{\frac{k z_{1}}{N}\right\},\left\{\frac{k z_{2}}{N}\right\}, \ldots,\left\{\frac{k z_{s}}{N}\right\}\right), k=1,2, \ldots, N
$$

where $N$ is the number of the nodes, $z$ is an $s$-dimensional generating vector of the lattice set and $\{a\}=a-[a]$ is the fractional part of $a$. The lattice rules with nodes (4) and generators $z$ are called "rank 1" rules. The class $E_{s}^{\alpha}(c)$ is defined by Koborov.

Definition 2: ([6]) We say that $f(x)$ belongs to the class of functions $E_{s}^{\alpha}(c)$ for $\alpha>1$ and $c>0$, if $f$ is a periodic function with period 1 for every of its components $x_{i}, i=$ $1,2 \ldots, s$, defined over the unit cube $[0,1]^{s}$ and its coefficients satisfy the following inequalities:

$$
|a(m)|<\frac{c}{\left(\bar{m}_{1} \ldots \bar{m}_{s}\right)^{\alpha}},
$$

where

$$
\bar{m}= \begin{cases}|m|, & |m| \neq 0 \\ 0, & m=0\end{cases}
$$

and the constant $c$ does not depend on $m_{1}, \ldots, m_{s}$.

Theorem 1: ([1]) There exists an optimal choice of the generating vector $z$, for which the error of integration satisfies

$$
\left|\frac{1}{N} \sum_{k=0}^{N-1} f\left(\left\{\frac{k}{N} z\right\}\right)-\int_{[0,1)^{s}} f(u) d u\right| \leq c d(s, \alpha) \frac{(\log N)^{\beta(s, \alpha)}}{N^{\alpha}},
$$

for the function $f \in E_{s}^{\alpha}(c)$, where $\alpha>1$ and $d(s, \alpha), \beta(s, \alpha)$ do not depend on $N$. Moreover, if $N$ is a prime number, then $\beta(s, \alpha)=\alpha(s-1)$.

The generating vector $z$, for which inequality (5) is satisfied, is an optimal generating vector in the sense of Korobov. The search over all possible $s$-dimensional lattice points $z \bmod n$, requires a significant computational effort in high dimensions. Thus, it is desirable to have efficient search schemes for finding the good lattice points. 
A. An optimized lattice rule based on an optimal generating vectors

For a given number of lattice points $N$, the performance of the routine is affected by the choice of the generator vector $z$. In 1981 Hua and Wang [5] generalized the Fibonacci numbers for any dimension. We refer to the lattice method with a generating vector

$$
z=\left(1, F_{l+1}^{(s)}, \ldots, F_{l+s-1}^{(s)}\right), \quad n_{l}=F_{l}^{(s)},
$$

where $F_{j}^{(s)}$ is the generalized Fibonacci number of dimension $s$, as Fibonacci lattice rule (FIBO). The advantages of the FIBO method are the linear complexity of the algorithm and fast computation of the generating vector. We propose an optimized lattice rule with a specific choice of the optimal 200-generating vector, constructed by Dirk Nuyens [7] in 2016 and the computation involves fast component by component operations. The method is improved by generating the points from a lattice sequence in base 2 in gray coded radical inverse ordering. The algorithm for reversing the digits of an integer number is computationally efficient. This optimal generating vector has been applied for the first time to a problem is computational finance and it gives superior results to the stochastic approaches used up to now. Since the performance of a lattice rule depends on the choice of the generator vectors, the 200-dimensional optimal vector is an optimization over the Fibonacci generalized vector (6).

\section{NUMERICAL EXAMPLES AND RESULTS}

The experimental results include the evaluation of the multidimensional integrals

$$
I_{s}=\int_{[0,1]^{s}} \exp \left(\prod_{i=1}^{s} x_{i}\right) d x_{1} \ldots d x_{s}
$$

By expanding the exponential function in Taylor series and integrating the terms $\left(x_{1} \cdots x_{s}\right)^{n}$ we obtain

$$
\begin{gathered}
\int_{[0,1]^{s}} \exp \left(\prod_{i=1}^{s} x_{i}\right)= \\
=\sum_{n=0}^{\infty} \frac{1}{(n+1)^{s} n !}={ }_{s} F_{s}(1, \cdots, 1 ; 2, \cdots, 2 ; 1),
\end{gathered}
$$

where ${ }_{p} F_{q}\left(a_{1}, \cdots, a_{p} ; b_{1}, \cdots, b_{q} ; x\right)$ is the generalized hypergeometric function

$$
{ }_{p} F_{q}\left(a_{1}, \cdots, a_{p} ; b_{1}, \cdots, b_{q} ; x\right)=\sum_{n=0}^{\infty} \frac{\left(a_{1}\right)_{n} \cdots\left(a_{p}\right)_{n}}{\left(b_{1}\right)_{n} \cdots\left(b_{q}\right)_{n}} \frac{x^{n}}{n !},
$$

and $(c)_{n}=c(c+1) \cdots(c+n-1)$ is the Pochhammer symbol.

$$
\begin{gathered}
\int_{[0,1]^{3}} \exp \left(x_{1} x_{2} x_{3}\right) \approx 1.14649907 . \\
\int_{[0,1]^{5}} \exp \left(\sum_{i=1}^{5} 0.5 a_{i} x_{i}^{2}\left(2+\sin \sum_{j=1, j \neq i}^{5} x_{j}\right)\right) \approx 2.923651,
\end{gathered}
$$

where $a_{i}=(1,0.5,0.2,0.2,0.2)$.

$$
\begin{gathered}
\int_{[0,1]^{8}} \exp \left(\sum_{i=1}^{8} 0.1 x_{i}\right)=1.496805 . \\
\int_{[0,1]^{20}} \exp \left(\prod_{i=1}^{20} x_{i}\right) \approx 1.00000949634 .
\end{gathered}
$$

We also compare the performance of the methods on a 50 dimensional integral:

$$
I_{50}=\int_{[0,1]^{50}} \exp \left(\prod_{i=1}^{50} x_{i}\right)
$$

We evaluate his reference value by expanding the exponential function in Taylor series and integrating the terms $\left(x_{1} \cdots x_{50}\right)^{n}$ we obtain

$$
\begin{gathered}
\int_{[0,1]^{50}} \exp \left(\prod_{i=1}^{50} x_{i}\right)= \\
=\sum_{n=0}^{\infty} \frac{1}{(n+1)^{50} n !}={ }_{50} F_{50}(1, \cdots, 1 ; 2, \cdots, 2 ; 1),
\end{gathered}
$$

where ${ }_{p} F_{q}\left(a_{1}, \cdots, a_{p} ; b_{1}, \cdots, b_{q} ; x\right)$ is the generalized hypergeometric function

$$
{ }_{p} F_{q}\left(a_{1}, \cdots, a_{p} ; b_{1}, \cdots, b_{q} ; x\right)=\sum_{n=0}^{\infty} \frac{\left(a_{1}\right)_{n} \cdots\left(a_{p}\right)_{n}}{\left(b_{1}\right)_{n} \cdots\left(b_{q}\right)_{n}} \frac{x^{n}}{n !},
$$

and $(c)_{n}=c(c+1) \cdots(c+n-1)$ is the Pochhammer symbol.

The experimental results are presented in the Tables below. Each table includes the relative error of the QMC method, the CPU-time and the number of integration nodes. We compare the performance of the optimized lattice rule (OPT), the Fibonacci based lattice rule (FIBO) and two other stochastic approaches Adapt for the wellknown adaptive stochastic approach and Sobol for the well know Sobol quasi-random sequence.

Table I

THE RELATIVE ERROR FOR 3 DIMENSIONAL INTEGRAL

\begin{tabular}{|c|c|c|c|c|c|c|c|c|}
\hline $\mathrm{N}$ & OPT & time & Adapt & time & FIBO & time & Sobol & time \\
\hline 19513 & $1.93 \mathrm{e}-5$ & 0.01 & $3.21 \mathrm{e}-4$ & 2.21 & $4.69 \mathrm{e}-4$ & 0.02 & $4.98 \mathrm{e}-5$ & 0.56 \\
35890 & $3.18 \mathrm{e}-6$ & 0.04 & $6.55 \mathrm{e}-5$ & 6.41 & $5.46 \mathrm{e}-6$ & 0.06 & $1.56 \mathrm{e}-5$ & 1.45 \\
66012 & $2.65 \mathrm{e}-6$ & 0.07 & $5.12 \mathrm{e}-5$ & 9.86 & $5.34 \mathrm{e}-6$ & 0.11 & $8.11 \mathrm{e}-6$ & 2.31 \\
121415 & $9.16 \mathrm{e}-7$ & 0.12 & $5.11 \mathrm{e}-5$ & 15.4 & $5.34 \mathrm{e}-6$ & 0.12 & $3.08 \mathrm{e}-6$ & 3.80 \\
223317 & $8.01 \mathrm{e}-7$ & 0.20 & $9.34 \mathrm{e}-5$ & 24.2 & $1.73 \mathrm{e}-6$ & 0.22 & $2.05 \mathrm{e}-6$ & 6.13 \\
\hline
\end{tabular}

Table II

THE RELATIVE ERROR FOR 3 DIMENSIONAL INTEGRAL

\begin{tabular}{|c|c|c|c|c|}
\hline time,s & OPT & Adapt & FIBO & sobol \\
\hline 0.1 & $9.16 \mathrm{e}-7$ & $8.67 \mathrm{e}-4$ & $1.32 \mathrm{e}-6$ & $3.21 \mathrm{e}-4$ \\
1 & $6.37 \mathrm{e}-7$ & $2.96 \mathrm{e}-5$ & $3.22 \mathrm{e}-7$ & $8.21 \mathrm{e}-5$ \\
2 & $4.22 \mathrm{e}-7$ & $5.45 \mathrm{e}-4$ & $2.06 \mathrm{e}-7$ & $2.96 \mathrm{e}-5$ \\
5 & $1.84 \mathrm{e}-7$ & $1.14 \mathrm{e}-4$ & $1.47 \mathrm{e}-7$ & $5.00 \mathrm{e}-6$ \\
10 & $6.09 \mathrm{e}-8$ & $6.56 \mathrm{e}-5$ & $3.89 \mathrm{e}-7$ & $2.71 \mathrm{e}-6$ \\
20 & $1.57 \mathrm{e}-8$ & $2.04 \mathrm{e}-5$ & $1.53 \mathrm{e}-8$ & $1.88 \mathrm{e}-6$ \\
\hline
\end{tabular}

The Adaptive Monte Carlo gives the worst results, because its strength is when the integrand has some pecularities. Sobol is suitable for non-smooth functions and it is outperformed by the other methods in low dimensions (Tables I,II,III,IV). The accuracy 
Table III

THE RELATIVE ERROR FOR 5 DIMENSIONAL INTEGRAL

\begin{tabular}{|c|c|c|c|c|c|c|c|c|}
\hline $\mathrm{N}$ & OPT & time & Adapt & time & FIBO & time & Sobol & time \\
\hline 13624 & $6.72 \mathrm{e}-5$ & 0.02 & $1.89 \mathrm{e}-3$ & 2.33 & $9.59 \mathrm{e}-4$ & 0.03 & $1.76 \mathrm{e}-4$ & 0.56 \\
52656 & $1.53 \mathrm{e}-5$ & 0.06 & $2.31 \mathrm{e}-3$ & 6.18 & $6.96 \mathrm{e}-4$ & 0.06 & $5.05 \mathrm{e}-5$ & 1.45 \\
103519 & $8.48 \mathrm{e}-6$ & 0.09 & $2.01 \mathrm{e}-3$ & 9.94 & $8.72 \mathrm{e}-5$ & 0.13 & $2.70 \mathrm{e}-5$ & 2.52 \\
203513 & $6.25 \mathrm{e}-6$ & 0.15 & $3.42 \mathrm{e}-4$ & 16.2 & $8.04 \mathrm{e}-5$ & 0.25 & $7.57 \mathrm{e}-6$ & 6.07 \\
400096 & $8.16 \mathrm{e}-7$ & 0.40 & $9.12 \mathrm{e}-4$ & 45.6 & $7.26 \mathrm{e}-5$ & 0.50 & $2.52 \mathrm{e}-6$ & 10.63 \\
\hline
\end{tabular}

Table IV

TIMES FOR 5 DIMENSIONAL INTEGRAL

\begin{tabular}{|c|c|c|c|c|}
\hline time,s & OPT & Adapt & FIBO & sobol \\
\hline 0.1 & $3.07 \mathrm{e}-6$ & $1.34 \mathrm{e}-2$ & $7.26 \mathrm{e}-5$ & $8.22 \mathrm{e}-4$ \\
1 & $1.32 \mathrm{e}-6$ & $2.44 \mathrm{e}-3$ & $2.28 \mathrm{e}-5$ & $2.91 \mathrm{e}-4$ \\
5 & $1.13 \mathrm{e}-6$ & $4.93 \mathrm{e}-4$ & $5.94 \mathrm{e}-6$ & $1.71 \mathrm{e}-5$ \\
10 & $5.47 \mathrm{e}-7$ & $1.88 \mathrm{e}-3$ & $3.85 \mathrm{e}-7$ & $1.79 \mathrm{e}-5$ \\
20 & $3.52 \mathrm{e}-7$ & $2.71 \mathrm{e}-4$ & $7.49 \mathrm{e}-7$ & $4.96 \mathrm{e}-6$ \\
\hline
\end{tabular}

Table V

THE RELATIVE ERROR FOR 8 DIMENSIONAL INTEGRAL

\begin{tabular}{|c|c|c|c|c|c|c|c|c|}
\hline $\mathrm{N}$ & OPT & time & Adapt & time & FIBO & time & Sobol & time \\
\hline 16128 & $1.79 \mathrm{e}-6$ & 0.04 & $1.10 \mathrm{e}-5$ & 12.6 & $8.08 \mathrm{e}-4$ & 0.03 & $8.87 \mathrm{e}-5$ & 0.13 \\
32192 & $1.56 \mathrm{e}-6$ & 0.05 & $3.32 \mathrm{e}-5$ & 33.3 & $1.03 \mathrm{e}-4$ & 0.07 & $5.42 \mathrm{e}-5$ & 0.58 \\
64256 & $8.01 \mathrm{e}-7$ & 0.08 & $4.65 \mathrm{e}-5$ & 54.2 & $5.03 \mathrm{e}-5$ & 0.11 & $2.34 \mathrm{e}-5$ & 2.49 \\
128257 & $6.22 \mathrm{e}-7$ & 0.13 & $8.25 \mathrm{e}-6$ & 88.3 & $8.13 \mathrm{e}-6$ & 0.14 & $4.45 \mathrm{e}-6$ & 6.36 \\
510994 & $3.21 \mathrm{e}-7$ & 0.34 & $7.07 \mathrm{e}-6$ & 233.6 & $5.95 \mathrm{e}-6$ & 0.57 & $3.32 \mathrm{e}-6$ & 19.45 \\
\hline
\end{tabular}

Table VI

TIMES FOR THE 8 DIMENSIONAL INTEGRAL

\begin{tabular}{|c|c|c|c|c|}
\hline time,s & OPT & Adapt & FIBO & sobol \\
\hline 1 & $2.18 \mathrm{e}-7$ & $6.34 \mathrm{e}-4$ & $5.34 \mathrm{e}-6$ & $2.02 \mathrm{e}-5$ \\
2 & $1.32 \mathrm{e}-7$ & $1.58 \mathrm{e}-4$ & $2.57 \mathrm{e}-6$ & $2.73 \mathrm{e}-5$ \\
5 & $9.03 \mathrm{e}-8$ & $1.44 \mathrm{e}-4$ & $1.52 \mathrm{e}-7$ & $8.88 \mathrm{e}-6$ \\
10 & $5.00 \mathrm{e}-8$ & $6.61 \mathrm{e}-5$ & $3.45 \mathrm{e}-6$ & $5.23 \mathrm{e}-6$ \\
20 & $2.55 \mathrm{e}-8$ & $2.77 \mathrm{e}-5$ & $1.82 \mathrm{e}-7$ & $2.11 \mathrm{e}-6$ \\
\hline
\end{tabular}

Table VII

THE RELATIVE ERROR FOR 20 DIMENSIONAL INTEGRAL

\begin{tabular}{|c|c|c|c|c|c|c|c|c|}
\hline $\mathrm{N}$ & OPT & time & Adapt & time & FIBO & time & Sobol & time \\
\hline 2048 & $2.84 \mathrm{e}-6$ & 0.02 & $1.14 \mathrm{e}-2$ & 8.6 & $8.22 \mathrm{e}-5$ & 0.03 & $8.44 \mathrm{e}-4$ & 0.13 \\
16384 & $1.04 \mathrm{e}-6$ & 0.12 & $4.96 \mathrm{e}-4$ & 60.3 & $3.12 \mathrm{e}-5$ & 0.13 & $6.82 \mathrm{e}-5$ & 1.68 \\
65536 & $9.21 \mathrm{e}-7$ & 0.91 & $9.75 \mathrm{e}-4$ & 474.2 & $1.36 \mathrm{e}-5$ & 1.17 & $8.34 \mathrm{e}-6$ & 8.69 \\
131072 & $6.15 \mathrm{e}-7$ & 2.13 & $1.25 \mathrm{e}-5$ & 888.3 & $8.85 \mathrm{e}-6$ & 2.34 & $3.77 \mathrm{e}-6$ & 14.36 \\
524288 & $5.33 \mathrm{e}-8$ & 8.13 & $1.96 \mathrm{e}-6$ & 2356 & $2.15 \mathrm{e}-6$ & 8.34 & $1.91 \mathrm{e}-7$ & 57 \\
\hline
\end{tabular}

Table VIII

TIMES FOR THE 20 DIMENSIONAL INTEGRAL

\begin{tabular}{|c|c|c|c|c|}
\hline time,s & OPT & Adapt & FIBO & sobol \\
\hline 1 & $9.14 \mathrm{e}-7$ & $1.58 \mathrm{e}-3$ & $1.48 \mathrm{e}-5$ & $3.25 \mathrm{e}-5$ \\
2 & $1.08 \mathrm{e}-7$ & $1.028 \mathrm{e}-3$ & $9.17 \mathrm{e}-6$ & $3.97 \mathrm{e}-5$ \\
5 & $5.87 \mathrm{e}-8$ & $8.58 \mathrm{e}-4$ & $5.19 \mathrm{e}-6$ & $1.45 \mathrm{e}-5$ \\
10 & $3.56 \mathrm{e}-8$ & $4.02 \mathrm{e}-4$ & $1.73 \mathrm{e}-6$ & $2.71 \mathrm{e}-6$ \\
20 & $1.23 \mathrm{e}-8$ & $1.13 \mathrm{e}-4$ & $1.38 \mathrm{e}-7$ & $1.76 \mathrm{e}-6$ \\
\hline
\end{tabular}

of the FIBO method and it has comparable execution time (Table 2). The Fibonacci lattice rule is the fastest method but its accuracy is lower than the accuracy of the lattice rules with increasing the dimensionality of the integral (Tables V,VI) and Sobol QMC becomes more accurate than FIBO (Tables VII,VIII). In highest dimension the optimized stochastic approach OPT has the lowest
Table IX

THE RELATIVE ERROR FOR 50 DIMENSIONAL INTEGRAL

\begin{tabular}{|c|c|c|c|c|c|c|}
\hline $\mathrm{N}$ & OPT & time & FIBO & time & Sobol & time \\
\hline $2^{10}$ & $2.88 \mathrm{e}-7$ & 0.05 & $6.23 \mathrm{e}-4$ & 0.08 & $8.88 \mathrm{e}-5$ & 3.5 \\
$2^{12}$ & $1.88 \mathrm{e}-7$ & 0.17 & $1.55 \mathrm{e}-4$ & 0.35 & $8.88 \mathrm{e}-5$ & 16 \\
$2^{16}$ & $5.44 \mathrm{e}-8$ & 2.14 & $9.72 \mathrm{e}-5$ & 5.21 & $8.88 \mathrm{e}-5$ & 73 \\
$2^{20}$ & $4.28 \mathrm{e}-8$ & 17.65 & $6.08 \mathrm{e}-5$ & 32.76 & $8.88 \mathrm{e}-6$ & 276 \\
\hline
\end{tabular}

Table X

TIMES FOR THE 50 DIMENSIONAL INTEGRAL

\begin{tabular}{|c|c|c|c|}
\hline time,s & OPT & FIBO & sobol \\
\hline 1 & $9.14 \mathrm{e}-8$ & $1.58 \mathrm{e}-3$ & $1.48 \mathrm{e}-5$ \\
2 & $3.68 \mathrm{e}-8$ & $1.028 \mathrm{e}-3$ & $9.17 \mathrm{e}-6$ \\
5 & $2.67 \mathrm{e}-8$ & $8.58 \mathrm{e}-4$ & $5.19 \mathrm{e}-6$ \\
10 & $3.34 \mathrm{e}-9$ & $4.02 \mathrm{e}-4$ & $1.73 \mathrm{e}-6$ \\
20 & $1.53 \mathrm{e}-9$ & $1.13 \mathrm{e}-4$ & $1.38 \mathrm{e}-7$ \\
\hline
\end{tabular}

relative error and a very high accuracy regardless of the dimension. (Table IX,X). The main advantage of the presented algorithms is their linear computational complexity with respect to dimension, while the deterministic methods suffer from the so-called curse of dimensionality and they become impractical in higher dimensions. The best performance of the optimized method is explained by the choice of the optimal generating vector, whose computation uses fast component-by-component operations. To summarize the optimized lattice rule gives the best results in terms of lowest relative errors and higher computational efficiency.

\section{CONCLUSION}

The applications of optimized lattice rules to the problem of option pricing is studied in the paper. The experimental results from Tables show that the optimized lattice rule with the special choice of the optimal generating vectors has the best performance with respect to relative error and computational time. The progress on the problem of option pricing and the computational finance area is closely related to the development of reliable algorithms for multidimensional numerical integration.

\section{REFERENCES}

[1] N. Bahvalov (1959) On the approximate calculation of multiple integrals, Journal of Complexity, Volume 31, Issue 4, 2015, Pages 502-516, ISSN 0885-064X, https://doi.org/10.1016/j.jco.2014.12.003.

[2] F. Black and M. Scholes (1973) The Pricing of Options and Corporate Liabilities, The Journal of Political Economy 81(3), 637-659, http://dx.doi.org/10.1086/260062.

[3] P.P. Boyle (1977) Options: a Monte Carlo Approach, Journal of Financial Economics 4(3), 323-338, https://doi.org/10.1016/0304405X(77)90005-8.

[4] Centeno, V., Georgiev, I. R., Mihova, V., \& Pavlov, V. (2019, October). Price forecasting and risk portfolio optimization. In AIP Conference Proceedings (Vol. 2164, No. 1, p. 060006). AIP Publishing LLC., https://doi.org/10.1063/1.5130808.

[5] L. K. Hua and Y. Wang, Applications of number theory to numerical analysis, (Springer 1981).

[6] N.M. Korobov, Number-theoretical methods in approximate analysis (Fizmatgiz, Moscow, 1963).

[7] Kuo, F.Y., Nuyens, D. Application of Quasi-Monte Carlo Methods to Elliptic PDEs with Random Diffusion Coefficients: A Survey of Analysis and Implementation. Found Comput Math 16, 16311696 (2016). https://doi.org/10.1007/s10208-016-9329-5.

[8] Lai Y., Spanier J. (2000) Applications of Monte Carlo/Quasi-Monte Carlo Methods in Finance: Option Pricing. In: Niederreiter H., Spanier J. (eds) Monte-Carlo and Quasi-Monte Carlo Methods 1998. Springer, Berlin, Heidelberg. https://doi.org/10.1007/978-3-642-59657-5_19 


\section{Optimized Quasi-Monte Carlo methods based on low discrepancy sequences for sensitivity analysis in air pollution modelling}

\author{
Venelin Todorov \\ Bulgarian Academy of Sciences \\ Institute of Mathematics and Informatics \\ ul. G. Bonchev 8, 1113 Sofia, Bulgaria \\ Bulgarian Academy of Sciences
}

Institute of Information and Communication Technologies

ul. G. Bonchev 25A, 1113 Sofia, Bulgaria

Email: vtodorov@math.bas.bg,venelin@parallel.bas.bg

\section{Ivan Dimov \\ Bulgarian Academy of Sciences}

Institute of Information and Communication Technologies

ul. G. Bonchev 25A, 1113 Sofia, Bulgaria

Email: ivdimov@bas.bg

\begin{abstract}
An optimization version of the van der Corput sequence has been used in our sensitivity studies of the model output results for some air pollutants with respect to the emission levels and some chemical reactions rates. Sensitivity analysis of model outputs to variation or natural uncertainties of model inputs is very significant for improving the reliability of these models. Clearly, the progress in the area of air pollution modeling, is closely connected with the progress in reliable algorithms for multidimensional integration.
\end{abstract}

\section{INTRODUCTON}

T HE main goal of the present work is to develop and investigate efficient stochastic algorithms for multiple numerical integration providing sensitivity analysis (SA) that means evaluating Sobol' sensitivity indices (SIs) [1], [3], [5]. While the classical deterministic grid methods are efficient for low dimensional integrands [2], they become computationally intensive and even impracticable for high dimensions $s$ because the number of required integrand evaluations grows exponentially. In contrast, the convergence rate of the plain Monte Carlo (MC) integration methods does not depend on the number of dimensions $s$. That is why the Monte Carlo method is a power tool in sensitivity analysis of large-scale systems [11].

Venelin Todorov is supported by the Bulgarian National Science Fund under Young Scientists Project KP-06-M32/2 - 17.12.2019 "Advanced Stochastic and Deterministic Approaches for Large-Scale Problems of Computational Mathematics" and by the National Scientific Program "Information and Communication Technologies for a Single Digital Market in Science, Education and Security (ICT in SES)", contract No DO1-205/23.11.2018, financed by the Ministry of Education and Science in Bulgaria. The work is also supported by the Bulgarian National Science Fund under Project DN 12/5-2017 "Efficient Stochastic Methods and Algorithms for Large-Scale Problems".

\author{
Tzvetan Ostromsky \\ Bulgarian Academy of Sciences \\ Institute of Information and Communication Technologies \\ ul. G. Bonchev 25A, 1113 Sofia, Bulgaria \\ Email: ceco@parallel.bas.bg
}

\author{
Stefka Fidanova \\ Bulgarian Academy of Sciences \\ Institute of Information and Communication Technologies \\ ul. G. Bonchev 25A, 1113 Sofia, Bulgaria \\ Email: stefka@parallel.bas.bg
}

The Unified Danish Eulerian Model (UNI-DEM)[12], [13] is in the focus of our investigation as one of the most advanced large-scale mathematical models that describes adequately all physical and chemical processes. UNI-DEM is a powerful large-scale air pollution model for calculation the concentrations of a large number of pollutants and other chemical species in the air. The calculations are done in a large spatial domain, which covers completely the European region and the Mediterranean, for certain time period (meteorological data must be available for it) [2]. In this particular study we use them for two of the most dangerous pollutants: the ozone $\left(\mathrm{O}_{3}\right)$ and the ammonia $\left(\mathrm{NH}_{3}\right)$. Other accumulative functions related to sone specific applications, maximal values, etc. are also calculated, exported and used in various application areas (environmental protection, agriculture, health care, etc.).

UNI-DEM is mathematically represented by the following system of partial differential equations (PDE), in which the unknown concentrations $c_{s}$ of a large number of chemical species (pollutants and other chemically active components) take part. The main physical and chemical processes (advection, diffusion, chemical reactions, emissions and deposition) are represented in that system:

$$
\begin{aligned}
\frac{\partial c_{s}}{\partial t}= & -\frac{\partial\left(u c_{s}\right)}{\partial x}-\frac{\partial\left(v c_{s}\right)}{\partial y}-\frac{\partial\left(w c_{s}\right)}{\partial z}+ \\
& +\frac{\partial}{\partial x}\left(K_{x} \frac{\partial c_{s}}{\partial x}\right)+\frac{\partial}{\partial y}\left(K_{y} \frac{\partial c_{s}}{\partial y}\right)+\frac{\partial}{\partial z}\left(K_{z} \frac{\partial c_{s}}{\partial z}\right)+(2) \\
& +E_{s}+Q_{s}\left(c_{1}, c_{2}, \ldots, c_{q}\right)-\left(k_{1 s}+k_{2 s}\right) c_{s}, s=1, \ldots, q(3)
\end{aligned}
$$


where $c_{s}$ are the concentrations of the chemical species; $u, v, w$ are the wind components along the coordinate axes; $K_{x}, K_{y}, K_{z}$ - the diffusion coefficients; $E_{s}$ - the emissions; $k_{1 s}, k_{2 s}-$ dry / wet deposition coefficients; $Q_{s}\left(c_{1}, c_{2}, \ldots c_{q}\right)$ - non-linear functions describing the chemical reactions between species under consideration. The above PDE system is non-linear and stiff. Both non-linearity and stiffness are introduced mainly by the chemical scheme: the condensed CBM-IV (Carbon Bond Mechanism) [13].

\section{THE VAN Der CoRput SEQUENCE}

Let $\mathbf{x}_{i}=\left(x_{i}^{(1)}, x_{i}^{(2)}, \ldots, x_{i}^{(s)}\right)$ for $i=1,2, \ldots$ The star discrepancy is given by:

$$
D_{N}^{*}=D_{N}^{*}\left(\mathbf{x}_{1}, \ldots, \mathbf{x}_{N}\right)=\sup _{\Omega \subset E^{s}}\left|\frac{\#\left\{\mathbf{x}_{n} \in \Omega\right\}}{N}-V(\Omega)\right|,
$$

where $E^{s}=[0,1)^{s}$. Let $m=\ldots a_{3}(m), a_{2}(m), a_{1}(m)$ be the representation of $m$ in base $b$. The radical inverse sequence is given by:

$$
m=\sum_{i=0}^{\infty} a_{i+1}(m) b^{i}, \phi_{b}(m)=\sum_{i=0}^{\infty} a_{i+1}(m) b^{-(i+1)}
$$

and it is fulfilled that

$$
D_{N}^{*}=O\left(\frac{\log N}{N}\right)
$$

The Van der Corput sequence is obtained when $b=2$ [9].

The van der Corput sequence is often used to generate a sequence of points which have a better covering property than pseudorandom points. The van der Corput sequence generates a sequence of points in $[0,1]$ which never repeats. The elements of the van der Corput sequence are strictly less than 1 . The van der Corput sequence writes an integer in a given base $b$, and then its digits are "reflected" about the decimal point. This maps the numbers from 1 to $\mathrm{N}$ into a set of numbers in $[0,1]$, which are especially nicely distributed if $N$ is one less than a power of the base. The generation is quite simple. Given an integer $I$, the expansion of $I$ in base $b$ is generated. Then, essentially, the result $R$ is generated by writing a decimal point followed by the digits of the expansion of $I$, in reverse order. This decimal value is actually still in base $b$, so it must be properly interpreted to generate a usable value. In the numerical experiments we will compare the standard van der Corput sequence VDC2 with an optimization variant when we increase the base to $b=2^{4}$ - we use the notation VDCO. Such comparison has been made for the first time for the particular model.

The lattice sequence that we are going to use in our experimental study, namely Fibonacci based lattice rule FIBO and polynomial lattice sequence are described in [6], [7], [10].

\section{Sensitivity Studies with Respect to Emission LEVELS}

In this section we will present the results for the sensitivity of UNI-DEM output (in particular, the ammonia mean monthly concentrations) with respect to the anthropogenic emissions input data variation are shown and discussed in this section. The anthropogenic emissions input consists of 4 different components

$$
\begin{aligned}
& \mathbf{E}^{\mathbf{A}} \text { - ammonia }\left(\mathrm{NH}_{3}\right) ; \\
& \mathbf{E}^{\mathbf{N}} \text { - nitrogen oxides }\left(\mathrm{NO}+\mathrm{NO}_{2}\right) \\
& \mathbf{E}^{\mathbf{S}} \text { - sulphur dioxide }\left(\mathrm{SO}_{2}\right) \\
& \mathbf{E}^{\mathbf{C}} \text { - anthropogenic hydrocarbons. }
\end{aligned}
$$

The domain under consideration is the 4-dimensional hypercube $\left.[0.5,1]^{4}\right)$. Polynomials of second degree have been used as an approximation tool. The input data have been generated by the improved SA-DEM version, developed for the purpose of our sensitivity studies (see the previous section).

Results of the relative error estimation for the quantities $f_{0}$, the total variance $\mathbf{D}$, first-order $\left(S_{i}\right)$ and total $\left(S_{i}^{t o t}\right)$ sensitivity indices are given in Tables I, II, III, respectively. $f_{0}$ is presented by a 4-dimensional integral, while the rest of the above quantities are presented by 8 -dimensional integrals, following the ideas of correlated sampling technique to compute sensitivity measures in a reliable way [8]. The four different stochastic approaches used for numerical integration are presented in separate columns of the tables.

A study of the computational efficiency of the stochastic algorithms under consideration for evaluating sensitivity measures presented by multidimensional integrals (total variance) or a ratio of multidimensional integrals (Sobol global sensitivity indices) have been made. The results show that the computational efficiency of the algorithms depends on integrand dimension and magnitude of estimated quantity. The order of relative error is different for different quantities of interest (see column Reference value) for the same sample size.

When $n=2^{16}$ it can be seen that the modified lattice rule LATM produce better results than the lattice rule based on Fibonacci generalized numbers FIBO - see Table III. The optimized van der Corput sequence produce worse results than both of the lattice sequences FIBO and LATM. It can be seen that the van der Corput sequence with base $b=2$ gives worse results by at least 1-2 orders to the optimized van der Corput sequence with base $b=2^{4}$ VDCO.

Most influential emissions about ammonia output concentrations are ammonia emissions themselves (about 89\% for Milan). The second most influential emissions about ammonia output are sulphur dioxide emissions (about 11\%).

\section{Sensitivity Studies with Respect to Chemical REACTIONS RATES}

In this section we will study the sensitivity of the ozone concentration values in the air over Genova with respect to the rate variation of some chemical reactions of the condensed CBM-IV scheme ([12]), namely: \# 1,3, 7,22 (time-dependent) 
TABLE I

RELATIVE ERROR FOR THE EVALUATION OF $f_{0} \approx 0.048$.

\begin{tabular}{|c|c|c|c|c|}
\hline & VDC2 & VDCO & FIBO & LATM \\
\hline $\begin{array}{l}\text { \# of samples } \\
n\end{array}$ & $\begin{array}{l}\text { Relative } \\
\text { error }\end{array}$ & $\begin{array}{l}\text { Relative } \\
\text { error }\end{array}$ & $\begin{array}{l}\text { Relative } \\
\text { error }\end{array}$ & $\begin{array}{l}\text { Relative } \\
\text { error }\end{array}$ \\
\hline $2^{10}$ & $3.72 \mathrm{e}-04$ & $1.64 \mathrm{e}-05$ & $2.09 \mathrm{e}-04$ & $8.46 \mathrm{e}-04$ \\
\hline $2^{12}$ & $4.39 \mathrm{e}-04$ & $5.11 \mathrm{e}-05$ & $4.32 \mathrm{e}-05$ & $1.79 \mathrm{e}-04$ \\
\hline $2^{14}$ & $2.15 \mathrm{e}-03$ & $4.45 \mathrm{e}-05$ & $2.25 \mathrm{e}-05$ & $2.62 \mathrm{e}-06$ \\
\hline $2^{16}$ & $3.11 \mathrm{e}-04$ & $5.56 \mathrm{e}-06$ & $8.70 \mathrm{e}-06$ & $4.14 \mathrm{e}-07$ \\
\hline $2^{18}$ & $7.66 e-05$ & $1.03 \mathrm{e}-06$ & $1.79 \mathrm{e}-06$ & $1.17 \mathrm{e}-06$ \\
\hline $2^{20}$ & $1.48 \mathrm{e}-04$ & $3.56 \mathrm{e}-07$ & $4.21 \mathrm{e}-07$ & $1.15 \mathrm{e}-06$ \\
\hline
\end{tabular}

TABLE II

RELATIVE ERROR FOR THE EVALUATION OF THE TOTAL VARIANCE $\mathbf{D} \approx 0.0002$.

\begin{tabular}{ccccc}
\hline \multirow{2}{*}{$\begin{array}{c}\text { \# of samples } \\
n\end{array}$} & VDC2 & VDCO & FIBO & LATM \\
\cline { 2 - 5 } & $\begin{array}{c}\text { Relative } \\
\text { error }\end{array}$ & $\begin{array}{c}\text { Relative } \\
\text { error }\end{array}$ & $\begin{array}{c}\text { Relative } \\
\text { error }\end{array}$ & $\begin{array}{c}\text { Relative } \\
\text { error }\end{array}$ \\
\hline \hline $2^{10}$ & $9.21 \mathrm{e}-02$ & $1.78 \mathrm{e}-02$ & $1.63 \mathrm{e}-01$ & $1.54 \mathrm{e}-02$ \\
$2^{12}$ & $6.37 \mathrm{e}-02$ & $4.11 \mathrm{e}-03$ & $2.39 \mathrm{e}-02$ & $3.67 \mathrm{e}-03$ \\
$2^{14}$ & $4.26 \mathrm{e}-02$ & $1.34 \mathrm{e}-03$ & $2.90 \mathrm{e}-03$ & $1.49 \mathrm{e}-03$ \\
$2^{16}$ & $2.22 \mathrm{e}-03$ & $3.19 \mathrm{e}-04$ & $2.65 \mathrm{e}-04$ & $1.61 \mathrm{e}-03$ \\
$2^{18}$ & $2.22 \mathrm{e}-03$ & $1.13 \mathrm{e}-04$ & $3.01 \mathrm{e}-04$ & $1.48 \mathrm{e}-03$ \\
$2^{20}$ & $7.58 \mathrm{e}-03$ & $5.76 \mathrm{e}-05$ & $1.19 \mathrm{e}-04$ & $1.46 \mathrm{e}-03$ \\
\hline
\end{tabular}

and \# 27,28 (time independent). The simplified chemical equations of those reactions are:

$$
\begin{array}{cl}
{[\# \mathbf{H 1}]} & \mathrm{NO}_{2}+\mathrm{h} \mathrm{N} \Longrightarrow \mathrm{NO}+\mathrm{O} ; \\
{[\mathrm{H3}]} & \mathrm{O}_{3}+\mathrm{NO} \Longrightarrow \mathrm{NO}_{2} ; \\
{[\mathrm{H7}]} & \mathrm{NO}_{2}+\mathrm{O}_{3} \Longrightarrow \mathrm{NO}_{3} ; \\
{[\# \mathbf{2 2}]} & \mathrm{HO}_{2}+\mathrm{NO} \Longrightarrow \mathrm{OH}+\mathrm{NO}_{2} ; \\
{[\# \mathbf{2 7}]} & \mathrm{HO}_{2}+\mathrm{HO}_{2} \Longrightarrow \mathrm{H}_{2} \mathrm{O}_{2} ; \\
{[\# \mathbf{2 8}]} & \mathrm{OH}+\mathrm{CO} \Longrightarrow \mathrm{HO}_{2}
\end{array}
$$

The domain under consideration is the 6-dimensional hypercube $\left.[0.6,1.4]^{6}\right)$.

Homma and Saltelli discuss in [4] which is the better estimation of

$$
f_{0}^{2}=\left(\int_{U^{d}} f(\mathbf{x}) \mathrm{d} \mathbf{x}\right)^{2}
$$

TABLE III

RELATIVE ERROR FOR ESTIMATION OF SENSITIVITY INDICES OF INPUT PARAMETERS USING VARIOUS MONTE CARLO AND QUASI-MONTE CARLO APPROACHES $(n \approx 65536)$.

\begin{tabular}{lccccc}
\hline Est. qnt. & Ref. val. & VDC2 & VDCO & FIBO & LATM \\
\hline$S_{1}$ & $9 \mathrm{e}-01$ & $3.13 \mathrm{e}-02$ & $4.56 \mathrm{e}-04$ & $3.62 \mathrm{e}-04$ & $7.27 \mathrm{e}-04$ \\
$S_{2}$ & $2 \mathrm{e}-04$ & $1.28 \mathrm{e}+00$ & $3.34 \mathrm{e}-02$ & $1.74 \mathrm{e}-01$ & $2.76 \mathrm{e}-02$ \\
$S_{3}$ & $1 \mathbf{e}-01$ & $9.13 \mathrm{e}-02$ & $2.22 \mathrm{e}-03$ & $3.22 \mathrm{e}-03$ & $4.24 \mathrm{e}-03$ \\
$S_{4}$ & $4 \mathbf{e}-05$ & $8.30 \mathrm{e}-01$ & $3.45 \mathrm{e}-02$ & $4.87 \mathrm{e}-01$ & $1.65 \mathrm{e}-02$ \\
\hline$S_{1}^{\text {tot }}$ & $9 \mathrm{e}-01$ & $7.54 \mathrm{e}-03$ & $1.16 \mathrm{e}-04$ & $4.61 \mathrm{e}-04$ & $5.14 \mathrm{e}-04$ \\
$S_{2}^{\text {tot }}$ & $2 \mathbf{e}-04$ & $4.69 \mathrm{e}+01$ & $1.24 \mathrm{e}-01$ & $3.45 \mathrm{e}-01$ & $2.21 \mathrm{e}-01$ \\
$S_{3}^{\text {tot }}$ & $1 \mathbf{e}-01$ & $4.14 \mathrm{e}-02$ & $1.10 \mathrm{e}-03$ & $1.96 \mathrm{e}-03$ & $6.41 \mathrm{e}-03$ \\
$S_{4}^{\text {tot }}$ & $5 \mathrm{e}-05$ & $5.54 \mathrm{e}+02$ & $1.60 \mathrm{e}-01$ & $5.06 \mathrm{e}-01$ & $1.60 \mathrm{e}-01$ \\
\hline
\end{tabular}

in the expression for total variance and Sobol global sensitivity measures. The first formula is

$$
f_{0}^{2} \approx \frac{1}{n} \sum_{i=1}^{n} f\left(\mathbf{x}_{i, 1}, \ldots, \mathbf{x}_{i, d}\right) f\left(\mathbf{x}_{i, 1}^{\prime}, \ldots, \mathbf{x}_{i, d}^{\prime}\right)
$$

and the second one is

$$
f_{0}^{2} \approx\left\{\frac{1}{n} \sum_{i=1}^{n} f\left(\mathbf{x}_{i, 1}, \ldots, \mathbf{x}_{i, d}\right)\right\}^{2}
$$

where $\mathbf{x}$ and $\mathbf{x}^{\prime}$ are two independent sample vectors. In case of estimating sensitivity indices of a fixed order, formula (8) is better (as recommended in [4]), here we use it too.

The relative error estimation for the quantities $f_{0}$, the total variance $\mathbf{D}$ and some sensitivity indices are given in Tables IV, V, VI respectively. The four different stochastic approaches used for numerical integration are presented in separate columns of the tables. The quantity $f_{0}$ is presented by 6 -dimensional integral, while the rest are presented by 12 dimensional integrals.

When $n=2^{16}$ it can be seen that the van der Corput sequences produce similar results to the modified lattice rule LATM and Fibonacci generalized numbers FIBO - see Table VI. When $n=2^{16}$ the optimized van der Corput sequence VDCO is far better than LATM and FIBO for same of the sensitivity indices - see the value for $S_{45}$ and $S_{12}$ in Table VI. Overall LATM produce better results than the Fibonacci based lattice rule by at least one order - see Table VI. None of the 4 methods estimates $S_{15}$ and $S_{5}$ reliably, which has extremely small reference values. This natural "size effect" does not destroy the accuracy of the corresponding total sensitivity indices (which are much larger, so the influence of $S_{5}$ and $S_{15}$ is negligible).

From these tables we can see the optimized van der Corput sequence gives better results than both the lattice sequences and the original van der Corput sequence with increasing the dimensionality of the integral. The results obtained by the optimized van der Corput sequence are better for 12 dimensional

\begin{tabular}{|c|c|c|c|c|}
\hline & VDC2 & VDCO & FIBO & LATM \\
\hline $\begin{array}{l}\# \text { of samples } \\
n\end{array}$ & $\begin{array}{l}\text { Relative } \\
\text { error }\end{array}$ & $\begin{array}{l}\text { Relative } \\
\text { error }\end{array}$ & $\begin{array}{l}\text { Relative } \\
\text { error }\end{array}$ & $\begin{array}{c}\text { Relative } \\
\text { error }\end{array}$ \\
\hline $2^{10}$ & $1.25 \mathrm{e}-02$ & $8.13 e-04$ & $2.08 \mathrm{e}-03$ & $7.12 \mathrm{e}-03$ \\
\hline $2^{12}$ & $1.70 \mathrm{e}-03$ & $2.12 \mathrm{e}-04$ & $1.40 \mathrm{e}-04$ & $1.80 \mathrm{e}-03$ \\
\hline $2^{14}$ & $3.56 \mathrm{e}-03$ & $6.15 \mathrm{e}-05$ & $3.98 \mathrm{e}-04$ & $4.04 \mathrm{e}-05$ \\
\hline $2^{16}$ & $8.66 \mathrm{e}-04$ & $7.09 \mathrm{e}-05$ & $2.61 \mathrm{e}-04$ & $9.91 \mathrm{e}-06$ \\
\hline $2^{18}$ & $4.63 \mathrm{e}-04$ & $2.33 \mathrm{e}-06$ & $7.29 \mathrm{e}-06$ & $7.24 \mathrm{e}-06$ \\
\hline $2^{20}$ & $7.85 \mathrm{e}-05$ & $3.51 \mathrm{e}-07$ & $4.57 \mathrm{e}-07$ & $7.04 \mathrm{e}-06$ \\
\hline
\end{tabular}
integrals compared with the results for 8 dimensional integrals. To summarize, Tthe algorithm has been successfully applied to compute global Sobol sensitivity measures corresponding to the influence of several input parameters (six chemical reactions rates and four different groups of pollutants) on the concentrations of important air pollutants.

TABLE IV

RELATIVE ERROR FOR THE EVALUATION OF $f_{0} \approx 0.27$ 
TABLE V

RELATIVE ERROR FOR THE EVALUATION OF THE TOTAL VARIANCE $\mathbf{D} \approx 0.0025$.

\begin{tabular}{ccccc}
\hline \multirow{3}{*}{$\begin{array}{c}\text { \# of samples } \\
n\end{array}$} & VDC2 & VDCO & FIBO & LATM \\
\cline { 2 - 5 } & $\begin{array}{c}\text { Relative } \\
\text { error }\end{array}$ & $\begin{array}{c}\text { Relative } \\
\text { error }\end{array}$ & $\begin{array}{c}\text { Relative } \\
\text { error }\end{array}$ & $\begin{array}{c}\text { Relative } \\
\text { error }\end{array}$ \\
\hline \hline $2^{10}$ & $2.23 \mathrm{e}-02$ & $1.10 \mathrm{e}-02$ & $6.73 \mathrm{e}+00$ & $3.11 \mathrm{e}-02$ \\
$2^{12}$ & $2.04 \mathrm{e}-01$ & $8.45 \mathrm{e}-03$ & $5.27 \mathrm{e}-01$ & $8.76 \mathrm{e}-02$ \\
$2^{14}$ & $3.94 \mathrm{e}-02$ & $6.44 \mathrm{e}-04$ & $1.02 \mathrm{e}-01$ & $7.54 \mathrm{e}-04$ \\
$2^{16}$ & $1.05 \mathrm{e}-03$ & $2.71 \mathrm{e}-04$ & $1.97 \mathrm{e}-03$ & $9.13 \mathrm{e}-04$ \\
$2^{18}$ & $1.27 \mathrm{e}-02$ & $1.01 \mathrm{e}-04$ & $4.53 \mathrm{e}-03$ & $2.22 \mathrm{e}-03$ \\
$2^{20}$ & $1.53 \mathrm{e}-02$ & $9.56 \mathrm{e}-05$ & $9.33 \mathrm{e}-03$ & $2.22 \mathrm{e}-03$ \\
\hline
\end{tabular}

TABLE VI

RELATIVE ERROR FOR ESTIMATION OF SENSITIVITY INDICES OF INPUT PARAMETERS USING VARIOUS QMC APPROACHES $(n \approx 65536)$.

\begin{tabular}{lccccc}
\hline Est. qnt. & Ref. val. & VDC2 & VDCO & FIBO & LATM \\
\hline$S_{1}$ & $4 \mathrm{e}-01$ & $1.07 \mathrm{e}-01$ & $1.92 \mathrm{e}-02$ & $3.82 \mathrm{e}-02$ & $1.50 \mathrm{e}-02$ \\
$S_{2}$ & $3 \mathrm{e}-01$ & $5.08 \mathrm{e}-02$ & $7.45 \mathrm{e}-03$ & $1.03 \mathrm{e}-02$ & $2.14 \mathrm{e}-02$ \\
$S_{3}$ & $5 \mathrm{e}-02$ & $6.37 \mathrm{e}-04$ & $5.22 \mathrm{e}-04$ & $5.48 \mathrm{e}-01$ & $8.28 \mathrm{e}-02$ \\
$S_{4}$ & $3 \mathrm{e}-01$ & $7.39 \mathrm{e}-02$ & $4.71 \mathrm{e}-03$ & $1.07 \mathrm{e}-02$ & $6.81 \mathrm{e}-03$ \\
$S_{5}$ & $4 \mathrm{e}-07$ & $7.26 \mathrm{e}+02$ & $9.00 \mathrm{e}+01$ & $3.40 \mathrm{e}+03$ & $2.07 \mathrm{e}+03$ \\
$S_{6}$ & $2 \mathrm{e}-02$ & $4.10 \mathrm{e}-01$ & $5.56 \mathrm{e}-03$ & $1.32 \mathrm{e}+00$ & $1.19 \mathrm{e}-02$ \\
\hline$S_{1}^{\text {tot }}$ & $4 \mathrm{e}-01$ & $8.89 \mathrm{e}-02$ & $6.56 \mathrm{e}-02$ & $7.92 \mathrm{e}-02$ & $1.07 \mathrm{e}-02$ \\
$S_{2}^{\text {tot }}$ & $3 \mathrm{e}-01$ & $1.06 \mathrm{e}-02$ & $1.41 \mathrm{e}-02$ & $3.06 \mathrm{e}-02$ & $2.28 \mathrm{e}-02$ \\
$S_{3}^{\text {tot }}$ & $5 \mathrm{e}-02$ & $1.25 \mathrm{e}-01$ & $5.13 \mathrm{e}-02$ & $1.31 \mathrm{e}+00$ & $4.92 \mathrm{e}-02$ \\
$S_{4}^{\text {tot }}$ & $3 \mathrm{e}-01$ & $1.51 \mathrm{e}-01$ & $8.23 \mathrm{e}-03$ & $3.84 \mathrm{e}-01$ & $1.93 \mathrm{e}-02$ \\
$S_{5}^{\text {tot }}$ & $2 \mathrm{e}-04$ & $3.45 \mathrm{e}+02$ & $3.02 \mathrm{e}+00$ & $8.85 \mathrm{e}+01$ & $6.78 \mathrm{e}+00$ \\
$S_{6}^{\text {tot }}$ & $2 \mathrm{e}-02$ & $1.63 \mathrm{e}+00$ & $4.87 \mathrm{e}-02$ & $2.15 \mathrm{e}+00$ & $7.63 \mathrm{e}-02$ \\
\hline$S_{12}$ & $6 \mathrm{e}-03$ & $9.16 \mathrm{e}-01$ & $1.34 \mathrm{e}-01$ & $3.21 \mathrm{e}+00$ & $2.21 \mathrm{e}-01$ \\
$S_{14}$ & $5 \mathrm{e}-03$ & $1.91 \mathrm{e}-01$ & $1.23 \mathrm{e}-01$ & $8.64 \mathrm{e}+00$ & $1.31 \mathrm{e}+00$ \\
$S_{15}$ & $8 \mathrm{e}-06$ & $8.45 \mathrm{e}+02$ & $1.72 \mathrm{e}+02$ & $9.19 \mathrm{e}+02$ & $9.62 \mathrm{e}+02$ \\
$S_{24}$ & $3 \mathrm{e}-03$ & $3.25 \mathrm{e}-01$ & $1.88 \mathrm{e}-02$ & $1.37 \mathrm{e}+01$ & $5.63 \mathrm{e}-01$ \\
$S_{45}$ & $1 \mathbf{e}-05$ & $1.41 \mathrm{e}-01$ & $4.23 \mathrm{e}-02$ & $4.25 \mathrm{e}+01$ & $3.87 \mathrm{e}+01$ \\
\hline
\end{tabular}

\section{CONCLUSION}

The computational efficiency (in terms of relative error and computational time) of several stochastic algorithms for multidimensional numerical integration has been studied to analyze the sensitivity of UNI-DEM model output to variation of input emissions of the anthropogenic pollutants and of rates of several chemical reactions. The algorithms have been successfully applied to compute global Sobol sensitivity measures corresponding to the influence of several input parameters on the concentrations of important air pollutants. The study has been done for the areas of several European cities with different geographical locations. The novelty of the proposed approaches is that the van der Corput sequence and its optimized version have been applied for the first time to sensitivity studies of the particular air pollution model. This is also the first time the Fibonacci based lattice rule and the modified lattice sequence based on transformation function are compared with the van der Corput sequence and its optimized version to the problem under consideration. The numerical tests show that the presented optimized stochastic approach is the most efficient for the multidimensional integrals under consideration and especially for computing small by value sensitivity indices.

\section{REFERENCES}

[1] G. Dimitriu: Global Sensitivity Analysis for a Chronic Myelogenous Leukemia Model: Proc. 9th International Conference NMA'2018, Borovets, Bulgaria, August 20-24, 2018, LNCS 11189, Springer, Jan 2019. DOI: $10.1007 / 978-3-030-10692-842$

[2] I. Dimov, R. Georgieva, Monte Carlo algorithms for evaluating Sobol' sensitivity indices, Mathematics and Computers in Simulation, Volume 81, Issue 3, 2010, Pages 506-514, ISSN 0378-4754, https://doi.org/10.1016/j.matcom.2009.09.005.

[3] H. Hamdad, Ch. Pézerat, B. Gauvreau, Ch. Locqueteau, Y. Denoual, Sensitivity analysis and propagation of uncertainty for the simulation of vehicle pass-by noise, Applied Acoustics Vol. 149, Elsevier, pp. 85-98 (June 2019). DOI: 10.1016/j.apacoust.2019.01.026

[4] T. Homma, A. Saltelli, Importance Measures in Global Sensitivity Analysis of Nonlinear Models, Reliability Engineering and System Safety 52, 1996, 1-17, https://doi.org/10.1016/0951-8320(96)00002-6.

[5] S. Poryazov. A suitable unit of sensitivity in telecommunications TELECOM 2011, 13-14.10.2011, Sofia, ISSN 1314-2690, p. 165-172.

[6] Sloan I.H. and Kachoyan P.J., Lattice methods for multiple integration: Theory, error analysis and examples, SIAM J. Numer. Anal. 24, pp. 116-128, 1987, https://doi.org/10.1137/0724010.

[7] I.H. Sloan and S. Joe, Lattice methods for multiple Integration, (Oxford University Press 1994).

[8] Sobol I.M., Tarantola S., Gatelli D., Kucherenko S., Mauntz W., Estimating the approximation error when fixing unessential factors in global sensitivity analysis, Reliability Engineering \& System Safety, 2007, 92, 957-960, https://doi.org/10.1016/j.ress.2006.07.001.

[9] van der Corput, J.G. (1935), "Verteilungsfunktionen (Erste Mitteilung)" (PDF), Proceedings of the Koninklijke Akademie van Wetenschappen te Amsterdam (in German), 38: 813-821, Zbl 0012.34705

[10] Wang Y., Hickernell F.J. (2002) An Historical Overview of Lattice Point Sets. In: Fang KT., Niederreiter H., Hickernell F.J. (eds) Monte Carlo and Quasi-Monte Carlo Methods 2000. Springer, Berlin, Heidelberg. https://doi.org/10.1007/978-3-642-56046-0_10.

[11] Zheleva, I., Georgiev, I., Filipova, M., \& Menseidov, D. (2017, October). Mathematical modeling of the heat transfer during pyrolysis process used for end-of-life tires treatment. In AIP Conference Proceedings (Vol. 1895, No. 1, p. 030008). AIP Publishing LLC, https://doi.org/10.1063/1.5007367.

[12] Z. Zlatev, Computer treatment of large air pollution models, KLUWER Academic Publishers, Dorsrecht-Boston-London, 1995.

[13] Z. Zlatev, I. T. Dimov, Computational and Numerical Challenges in Environmental Modelling, Elsevier, Amsterdam, 2006. 


\section{Sensitivity Study of a Large-Scale Air Pollution Model by Using Optimized Stochastic Algorithm}

\author{
Venelin Todorov \\ Bulgarian Academy of Sciences \\ Institute of Mathematics and Informatics \\ ul. G. Bonchev 8, 1113 Sofia, Bulgaria \\ Bulgarian Academy of Sciences \\ Institute of Information and Communication Technologies \\ ul. G. Bonchev 25A, 1113 Sofia, Bulgaria \\ Email: vtodorov@math.bas.bg,venelin@ parallel.bas.bg

\section{Ivan Dimov} \\ Bulgarian Academy of Sciences \\ Institute of Information and Communication Technologies \\ ul. G. Bonchev 25A, 1113 Sofia, Bulgaria \\ Email: ivdimov@bas.bg
}

\author{
Tzvetan Ostromsky \\ Bulgarian Academy of Sciences \\ Institute of Information and Communication Technologies \\ ul. G. Bonchev 25A, 1113 Sofia, Bulgaria \\ Email: ceco@parallel.bas.bg
}

\begin{abstract}
In this work a systematic procedure for multidimensional sensitivity analysis in the area of air pollution modeling by an optimized latin hypercube sampling has been done. The Unified Danish Eulerian Model (UNI-DEM) is used in our investigation, because this is one of the most advanced largescale mathematical models that describes adequately all physical and chemical processes. We study the sensitivity of concentration variations of some of the most dangerous air pollutants with respect to the anthropogenic emissions levels and with respect to some chemical reactions rates. The results obtained with an adaptive approach and latin hypercube sampling has been discussed.
\end{abstract}

\section{INTRODUCTION}

$\mathbf{H}$ ERE we discuss a systematic approach for sensitivity analysis studies in the area of air pollution modelling. The Unified Danish Eulerian Model (UNI-DEM) [14], [15] is used in this particular study. Different parts of the large amount of output data, produced by the model, were used in various practical applications, where the reliability of this data should be properly estimated [11], [13]. Another reason to choose this model as a case study here is its sophisticated chemical scheme, where all relevant chemical processes in the atmosphere are accurately represented. We study the sensitivity of concentration variations of some of the most dangerous air pollutants with respect to the anthropogenic emissions levels and with respect to some chemical reactions rates.

This work is supported by the Bulgarian National Science Fund under Project DN 12/5-2017. Venelin Todorov is supported by the Bulgarian National Science Fund under Project KP-06-M32/2-17.12.2019 "Advanced Stochastic and Deterministic Approaches for Large-Scale Problems of Computational Mathematics" and by the National Scientific Program "Information and Communication Technologies for a Single Digital Market in Science, Education and Security (ICT in SES)", contract No D01-205/23.11.2018, financed by the Ministry of Education and Science in Bulgaria.
Sensitivity studies are nowadays applied to some of the most complicated mathematical models from various intensively developing areas of application ([1], [6]). Different efficient stochastic algorithms for multidimensional integration have also been applied on a further stage of these sensitivity studies. The two Adaptive Monte Carlo algorithms that we are going to used are based on the ideas and results of the importance separation and are completely described in the following works [2], [3], [4], [5].

\section{OPtimized LATIN HyPERCUBE SAMPLING}

Latin Hypercube Sampling is a type of stratified sampling, described for the first time by M.D. McKay in 1979 in [9]. For this standard method we will use the notation LHS. Recently a renewing interest in optimization version of Latin hypercube sampling is described by Budiman Minasny in his works [10]. In the case of integral approximation we must simply divide the domain $[0,1]^{d}$ into $m^{d}$ disjoint subdomains, each of volume $\frac{1}{m^{d}}$ and to sample one point from each of them. Let this sample be $\mathbf{x}_{k, j}$, for dimensions $k=1, \ldots, m^{d}$, $j=1, \ldots, d$. LHS does not require more samples for more dimensions (variables) - it is one of the main advantages of this scheme. Examples of random, stratified and Latin hypercube samplings with 16 points are presented on Figure 1 [8].

Here we propose an optimized version of the Latin Hypercube Sampling which includes a uniformly distributed random point in each interval which improves the accuracy as can be seen from the results below. The description of the optimized latin hypercube sampling (LHSO) algorithm is the following:

1) Divide the distribution of each variable $X$ into $n$ equiprobable intervals. 


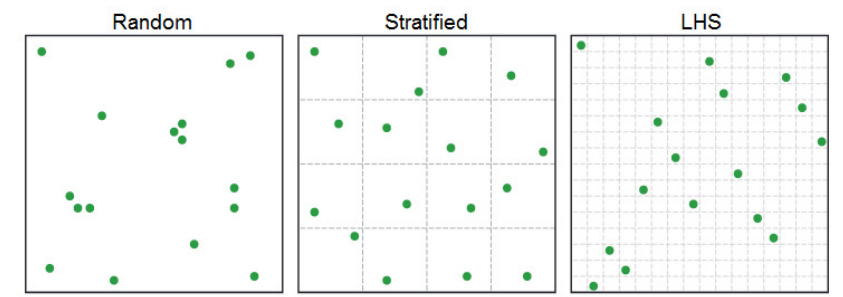

Fig. 1. Comparison of random, stratified and latin hypercube samplings with 16 points $(\mathrm{d}=2, \mathrm{M}=4)$.

2) For each interval, choose one uniform random number $r \in[0,1]$. Then, let

$$
\operatorname{Prob}_{i}=\frac{i-1}{n}+r * \frac{1}{n}
$$

3) Transform the probability into the sampled value $x$ using the inverse of the cumulative distribution function:

$$
x=F^{-1}(\text { Prob })
$$

4) The $n$ values obtained for each variable $X$ are paired randomly(e.g. by generating a random permutation of $\{1,2, \ldots, n\}$ for each variable except one) with the $n$ values of the other variables.

\section{SEnsitivity Studies With Respect to Emission LEVELS}

The huge output data stream of UNI-DEM contains are the mean monthly concentrations of more than 30 pollutants. We consider 2 of them: ozone $\left(\mathrm{O}_{3}\right)$ and ammonia $\left(\mathrm{NH}_{3}\right)$. In particular, we present some results of a sensitivity study of the mean monthly concentrations of ammonia.

Here we present some results of our research on the sensitivity of UNI-DEM output (in particular, the ammonia mean monthly concentrations) with respect to the anthropogenic emissions input variation. The anthropogenic emissions input consists of 4 different components $\mathbf{E}=\left(\mathbf{E}^{\mathbf{A}}, \mathbf{E}^{\mathbf{N}}, \mathbf{E}^{\mathbf{S}}, \mathbf{E}^{\mathbf{C}}\right)$ as follows:

$$
\begin{aligned}
& \mathbf{E}^{\mathbf{A}}-\text { ammonia }\left(\mathrm{NH}_{3}\right) ; \\
& \mathbf{E}^{\mathbf{N}}-\text { nitrogen oxides }\left(\mathrm{NO}+\mathrm{NO}_{2}\right) ; \\
& \mathbf{E}^{\mathbf{S}}-\text { sulphur dioxide }\left(\mathrm{SO}_{2}\right) ; \\
& \mathbf{E}^{\mathbf{C}}-\text { anthropogenic hydrocarbons. }
\end{aligned}
$$

The domain is the 4-dimensional hypercube $\left.[0.5,1]^{4}\right)$. The input data have been generated by the improved version SADEM code, specialized for sensitivity studies (see the previous section).

The results for relative errors for evaluation of the quantities $f_{0}$, total variances and first-order and total sensitivity indices using various stochastic approaches for numerical integration are presented in Tables I, II, III, respectively. The quantity $f_{0}$ is presented by a 4-dimensional integral whereas the rest of the quantities under consideration are presented by an 8 dimensional integrals. In this work for the estimateq quantity we use the notation EQ.
TABLE I

RELATIVE ERROR FOR THE EVALUATION OF $f_{0} \approx 0.048$.

\begin{tabular}{ccccc}
\hline \multirow{2}{*}{$\begin{array}{c}\text { \# of samples } \\
n\end{array}$} & ADAPT1 & ADAPT2 & LHSO & LHS \\
\cline { 2 - 5 } & $\begin{array}{c}\text { Relative } \\
\text { error }\end{array}$ & $\begin{array}{c}\text { Relative } \\
\text { error }\end{array}$ & $\begin{array}{c}\text { Relative } \\
\text { error }\end{array}$ & $\begin{array}{c}\text { Relative } \\
\text { error }\end{array}$ \\
\hline \hline $2^{16}$ & $9.89 \mathrm{e}-05$ & $4.05 \mathrm{e}-04$ & $1.45 \mathrm{e}-05$ & $7.74 \mathrm{e}-05$ \\
$2^{18}$ & $3.95 \mathrm{e}-05$ & $3.83 \mathrm{e}-06$ & $1.98 \mathrm{e}-06$ & $3.80 \mathrm{e}-06$ \\
$2^{20}$ & $4.99 \mathrm{e}-05$ & $2.93 \mathrm{e}-05$ & $6.31 \mathrm{e}-06$ & $7.16 \mathrm{e}-06$ \\
\hline
\end{tabular}

TABLE II

RELATIVE ERROR FOR THE EVALUATION OF THE TOTAL VARIANCE $\mathbf{D} \approx 0.0002$.

\begin{tabular}{ccccc}
\hline \multirow{2}{*}{$\begin{array}{c}\text { \# of samples } \\
n\end{array}$} & ADAPT1 & ADAPT2 & LHSO & LHS \\
\cline { 2 - 5 } & $\begin{array}{c}\text { Relative } \\
\text { error }\end{array}$ & $\begin{array}{c}\text { Relative } \\
\text { error }\end{array}$ & $\begin{array}{c}\text { Relative } \\
\text { error }\end{array}$ & $\begin{array}{c}\text { Relative } \\
\text { error }\end{array}$ \\
\hline \hline $2^{16}$ & $1.83 \mathrm{e}-04$ & $5.12 \mathrm{e}-04$ & $2.17 \mathrm{e}-04$ & $3.65 \mathrm{e}-04$ \\
$2^{18}$ & $5.77 \mathrm{e}-05$ & $1.21 \mathrm{e}-04$ & $4.56 \mathrm{e}-05$ & $1.21 \mathrm{e}-05$ \\
$2^{20}$ & $3.42 \mathrm{e}-05$ & $3.28 \mathrm{e}-05$ & $1.87 \mathrm{e}-05$ & $5.96 \mathrm{e}-05$ \\
\hline
\end{tabular}

The results show that the algorithms using LHSO simulate the behaviour of the Adaptive Monte Carlo algorithm, and for some small in value sensitivity indices as $S_{4}^{\text {tot }}$ LHSO gives better results than the adaptive approach. Adaptive algorithm gives results of the same order as LHS, and sometimes even outperforms it (see for example the relative errors for $S_{1}^{t o t}$ ).

\section{Sensitivity Studies with Respect to Chemical REACTIONS RATES}

We will also study the sensitivity of the ozone concentration values in the air over Genova with respect to the rate of variation of some chemical reactions of the condensed CBMIV scheme ([14]), namely: \# 1, 3, 7, 22 (time-dependent) and \# 27,28 (time independent). The simplified chemical equations of those reactions are as follows:

$$
\begin{aligned}
& \text { [\#1] } \mathrm{NO}_{2}+h \nu \Longrightarrow \mathrm{NO}+\mathrm{O} \text {; } \\
& \text { [\#3] } \mathrm{O}_{3}+\mathrm{NO} \Longrightarrow \mathrm{NO}_{2} \text {; } \\
& {[\# \mathbf{7}] \quad \mathrm{NO}_{2}+\mathrm{O}_{3} \Longrightarrow \mathrm{NO}_{3} \text {; }}
\end{aligned}
$$

TABLE III

RELATIVE ERROR FOR ESTIMATION OF SENSITIVITY INDICES OF THE INPUT ANTHROPOGENIC EMISSIONS BY USING VARIOUS MONTE CARLO APPROACHES $\left(n=2^{16}=65536\right)$.

\begin{tabular}{lccccc}
\hline EQ & Ref. value & ADAPT1 & ADAPT2 & LHSO & LHS \\
\hline$S_{1}$ & $9 \mathrm{e}-01$ & $7.67 \mathrm{e}-04$ & $1.22 \mathrm{e}-03$ & $1.16 \mathrm{e}-04$ & $9.79 \mathrm{e}-03$ \\
$S_{2}$ & $2 \mathbf{e}-04$ & $1.47 \mathrm{e}-03$ & $4.96 \mathrm{e}-02$ & $7.10 \mathrm{e}-03$ & $6.60 \mathrm{e}-01$ \\
$S_{3}$ & $1 \mathbf{e}-01$ & $4.11 \mathrm{e}-03$ & $1.59 \mathrm{e}-03$ & $1.19 \mathrm{e}-03$ & $8.65 \mathrm{e}-03$ \\
$S_{4}$ & $4 \mathrm{e}-05$ & $1.04 \mathrm{e}-01$ & $1.69 \mathrm{e}-01$ & $6.10 \mathrm{e}-02$ & $6.70 \mathrm{e}-01$ \\
\hline$S_{1}^{t o t}$ & $9 \mathrm{e}-01$ & $4.99 \mathrm{e}-05$ & $5.36 \mathrm{e}-05$ & $7.31 \mathrm{e}-05$ & $4.31 \mathrm{e}-04$ \\
$S_{2}^{\text {tot }}$ & $2 \mathbf{e}-04$ & $5.23 \mathrm{e}-01$ & $5.00 \mathrm{e}+00$ & $1.07 \mathrm{e}-01$ & $2.94 \mathrm{e}+01$ \\
$S_{3}^{\text {tot }}$ & $1 \mathbf{e}-01$ & $1.15 \mathrm{e}-02$ & $1.28 \mathrm{e}-02$ & $5.45 \mathrm{e}-03$ & $1.10 \mathrm{e}-02$ \\
$S_{4}^{\text {tot }}$ & $5 \mathbf{e}-05$ & $1.88 \mathrm{e}+01$ & $3.43 \mathrm{e}+01$ & $7.54 \mathrm{e}-01$ & $2.41 \mathrm{e}+02$ \\
\hline
\end{tabular}




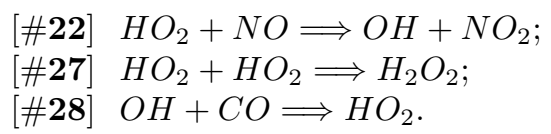

The domain under consideration is the 6-dimensional hypercube $\left.[0.6,1.4]^{6}\right)$.

Homma and Saltelli discuss in [7] which is the better estimation of $f_{0}^{2}=\left(\int_{U^{d}} f(\mathbf{x}) \mathrm{d} \mathbf{x}\right)^{2}$ in the expression for total variance and Sobol global sensitivity measures. In case of estimating sensitivity indices of a fixed order, the formula $f_{0}^{2} \approx \frac{1}{n} \sum_{i=1}^{n} f\left(\mathbf{x}_{i, 1}, \ldots, \mathbf{x}_{i, d}\right) f\left(\mathbf{x}_{i, 1}^{\prime}, \ldots, \mathbf{x}_{i, d}^{\prime}\right)$, where $\mathbf{x}$ and $\mathbf{x}^{\prime}$ are two independent sample vectors, is better (as recommended in [7], [12]).

TABLE IV

RELATIVE ERROR FOR EVALUATION OF $f_{0} \approx 0.27$

\begin{tabular}{ccccc}
\hline \multirow{2}{*}{$\begin{array}{c}\text { \# of samples } \\
n\end{array}$} & ADAPT1 & ADAPT2 & LHSR & LHS \\
\cline { 2 - 5 } & $\begin{array}{c}\text { Relative } \\
\text { error }\end{array}$ & $\begin{array}{c}\text { Relative } \\
\text { error }\end{array}$ & $\begin{array}{c}\text { Relative } \\
\text { error }\end{array}$ & $\begin{array}{c}\text { Relative } \\
\text { error }\end{array}$ \\
\hline \hline $2^{16}$ & $3.49 \mathrm{e}-05$ & $3.72 \mathrm{e}-05$ & $1.12 \mathrm{e}-05$ & $2.02 \mathrm{e}-04$ \\
$2^{18}$ & $5.90 \mathrm{e}-07$ & $1.02 \mathrm{e}-06$ & $6.14 \mathrm{e}-06$ & $2.82 \mathrm{e}-05$ \\
$2^{20}$ & $1.36 \mathrm{e}-07$ & $5.56 \mathrm{e}-07$ & $8.71 \mathrm{e}-07$ & $1.04 \mathrm{e}-05$ \\
\hline
\end{tabular}

The relative errors for evaluation of the quantities $f_{0}$, total variances, first and second order sensitivity indices by using various stochastic approaches for numerical integration are presented in Tables IV, V, VI respectively. Here the quantity $f_{0}$ is presented by a 6-dimensional integral, whereas the total variance and the sensitivity indices are presented by 12-dimensional integrals, following the ideas of correlated sampling.

TABLE V

RELATIVE ERROR FOR EVALUATION OF THE TOTAL VARIANCE $\mathbf{D} \approx 0.0025$

\begin{tabular}{ccccc}
\hline \multirow{2}{*}{$\begin{array}{c}\text { \# of samples } \\
n\end{array}$} & ADAPT1 & ADAPT2 & LHSO & LHS \\
\cline { 2 - 5 } & $\begin{array}{c}\text { Relative } \\
\text { error }\end{array}$ & $\begin{array}{c}\text { Relative } \\
\text { error }\end{array}$ & $\begin{array}{c}\text { Relative } \\
\text { error }\end{array}$ & $\begin{array}{c}\text { Relative } \\
\text { error }\end{array}$ \\
\hline \hline $2^{16}$ & $3.01 \mathrm{e}-05$ & $1.48 \mathrm{e}-04$ & $8.23 \mathrm{e}-05$ & $3.56 \mathrm{e}-05$ \\
$2^{18}$ & $7.71 \mathrm{e}-06$ & $3.79 \mathrm{e}-04$ & $6.12 \mathrm{e}-05$ & $7.78 \mathrm{e}-03$ \\
$2^{20}$ & $1.75 \mathrm{e}-06$ & $3.34 \mathrm{e}-05$ & $5.21 \mathrm{e}-05$ & $2.78 \mathrm{e}-04$ \\
\hline
\end{tabular}

TABLE VI

RELATIVE ERROR FOR ESTIMATION OF SENSITIVITY INDICES OF SEVERAL CHEMICAL REACTION RATE PARAMETERS BY USING VARIOUS MONTE CARLO AND QUASI-MONTE CARLO APPROACHES $\left(n=2^{16}=65536\right)$.

\begin{tabular}{lccccc}
\hline EQ & Ref. value & ADAPT1 & ADAPT2 & LHSO & LHS \\
\hline$S_{1}$ & $4 \mathrm{e}-01$ & $1.55 \mathrm{e}-04$ & $3.48 \mathrm{e}-04$ & $7.53 \mathrm{e}-05$ & $3.04 \mathrm{e}-02$ \\
$S_{2}$ & $3 \mathbf{e}-01$ & $4.34 \mathrm{e}-04$ & $1.58 \mathrm{e}-04$ & $5.19 \mathrm{e}-04$ & $7.35 \mathrm{e}-04$ \\
$S_{3}$ & $5 \mathrm{e}-02$ & $3.42 \mathrm{e}-04$ & $8.09 \mathrm{e}-05$ & $7.24 \mathrm{e}-04$ & $2.33 \mathrm{e}-02$ \\
$S_{4}$ & $3 \mathrm{e}-01$ & $4.75 \mathrm{e}-04$ & $9.04 \mathrm{e}-04$ & $4.31 \mathrm{e}-04$ & $2.47 \mathrm{e}-02$ \\
$S_{5}$ & $4 \mathrm{e}-07$ & $1.31 \mathrm{e}+01$ & $1.07 \mathrm{e}+01$ & $2.37 \mathrm{e}+01$ & $9.25 \mathrm{e}+02$ \\
$S_{6}$ & $2 \mathrm{e}-02$ & $1.08 \mathrm{e}-03$ & $4.54 \mathrm{e}-04$ & $5.21 \mathrm{e}-04$ & $3.81 \mathrm{e}-02$ \\
\hline$S_{12}$ & $6 \mathbf{e}-03$ & $1.30 \mathrm{e}-02$ & $7.92 \mathrm{e}-03$ & $7.70 \mathrm{e}-03$ & $8.99 \mathrm{e}-02$ \\
$S_{14}$ & $5 \mathrm{e}-03$ & $5.30 \mathrm{e}-03$ & $1.81 \mathrm{e}-03$ & $5.11 \mathrm{e}-03$ & $2.74 \mathrm{e}-01$ \\
$S_{15}$ & $8 \mathrm{e}-06$ & $9.34 \mathrm{e}+02$ & $9.34 \mathrm{e}+02$ & $9.06 \mathrm{e}+02$ & $9.21 \mathrm{e}+02$ \\
$S_{24}$ & $3 \mathbf{e}-03$ & $1.26 \mathrm{e}-03$ & $7.24 \mathrm{e}-03$ & $4.21 \mathrm{e}-03$ & $7.10 \mathrm{e}-01$ \\
$S_{45}$ & $1 \mathbf{e}-05$ & $9.93 \mathrm{e}-02$ & $8.55 \mathrm{e}-02$ & $1.14 \mathrm{e}-01$ & $1.05 \mathrm{e}+01$ \\
\hline
\end{tabular}

We can conclude that all stochastic approaches under consideration give reliable relative errors for sufficiently large number of samples. The adaptive MC algorithm outperforms LHS in the case of small number of samples, higher dimensions and small by value sensitivity indices. The algorithms using generalized LHSO simulate the behaviour of Adaptive Monte Carlo algorithm, and even for higher dimensions sometimes gives better results than the to adaptive approach by at least one order. The most efficient in terms of computational complexity is the LHSO algorithm, followed very closely by the LHS algorithm. The algorithm LHS is characterized with unreliable relative errors for small in value sensitivity measures. MC approach based on optimized Latin hypercube sampling produce significantly better results for 6 -dimensional and 12-dimensional integrals in comparison with the standard latin hypercube sampling LHS.

\section{CONCLUSION}

A comprehensive experimental study of Monte Carlo algorithm based on optimized latin hypercube sampling and adaptive approach for multidimensional numerical integration has been done. Such comparison has been made for the first time and this motivates the present study. The algorithms have been successfully applied to compute global Sobol sensitivity measures corresponding to the six chemical reactions rates and four different groups of pollutants. The numerical tests show that the optimized stochastic approach gives comparable results to the adaptive approach and better than the standard latin hypercube sampling and this method is very efficient for multidimensional numerical integration.

\section{REFERENCES}

[1] G. Dimitriu: Global Sensitivity Analysis for a Chronic Myelogenous Leukemia Model: Proc. 9th International Conference NMA'2018, Borovets, Bulgaria, August 20-24, 2018, LNCS 11189, Springer, Jan 2019. DOI: $10.1007 / 978-3-030-10692-8 \_42$

[2] I.T. Dimov, R. Georgieva. Monte Carlo Method for Numerical Integration based on Sobol' Sequences. Numerical Methods and Applications (I. Dimov, S. Dimova, N. Kolkovska - Eds.), LNCS 6046, Springer, 2011, 50-59, https://doi.org/10.1007/978-3-642-18466-6_5.

[3] I. T. Dimov, R. Georgieva. Multidimensional Sensitivity Analysis of Large-scale Mathematical Models. O.P. Iliev et al. (eds.), Numerical Solution of Partial Differential Equations: Theory, Algorithms, and Their Applications, Springer Proceedings in Mathematics \& Statistics 45, Springer Science+Business Media, New York, 2013, 137-156, https://doi.org/10.1007/978-1-4614-7172-1_8.

[4] Dimov I., Karaivanova A., Error analysis of an adaptive Monte Carlo method for numerical integration, Mathematics and Computers in simulation 47 pp. 201-213, 1998, https://doi.org/10.1016/S03784754(98)00103-7.

[5] Dimov I., Karaivanova A., Georgieva R., Ivanovska S. (2003) Parallel Importance Separation and Adaptive Monte Carlo Algorithms for Multiple Integrals, Springer Lecture Notes in Computer Science, 2542, Springer-Verlag, Berlin, Heidelberg, New York: 99-107, https://doi.org/10.1007/3-540-36487-0_10.

[6] H. Hamdad, Ch. Pézerat, B. Gauvreau, Ch. Locqueteau, Y. Denoual, Sensitivity analysis and propagation of uncertainty for the simulation of vehicle pass-by noise, Applied Acoustics Vol. 149, Elsevier, pp. 85-98 (June 2019). DOI: 10.1016/j.apacoust.2019.01.026

[7] T. Homma, A. Saltelli, Importance Measures in Global Sensitivity Analysis of Nonlinear Models, Reliability Engineering and System Safety 52, 1996, 1-17, https://doi.org/10.1016/0951-8320(96)00002-6.

[8] Jarosz, W.: Efficient Monte Carlo Methods for Light Transport in Scattering Media, PhD dissertation, UCSD, (2008). 
[9] McKay, M.D., Beckman, R.J., Conover, W.J - A comparison of three methods for selecting values of input variables in the analysis of output from a computer code. Technometrics 21(2), 239-45 (1979), https://www.jstor.org/stable/1268522?seq=1.

[10] Minasny B., McBratney B.: A conditioned Latin hypercube method for sampling in the presence of ancillary information, Journal Computer and Geosciences archive, Volume 32 Issue 9, November, 2006, Pages 1378-1388, https://doi.org/10.1016/j.cageo.2005.12.009.

[11] S. Poryazov, E. Saranova, and I. Ganchev. Conceptual and Analytical Models for Predicting the Quality of Service of Overall Telecommunication Systems. Autonomous Control for a Reliable Internet of Services. Springer, Cham, 2018, 151-181, https://doi.org/10.1007/9783-319-90415-3 7.
[12] Sobol IM. Tarantola S., Gatelli D., Kucherenko S, Mauntz W., Estimating the approximation error when fixing unessential factors in global sensitivity analysis, Reliability Engineering \& System Safety, 2007, 92, 957-960, https://doi.org/10.1016/j.ress.2006.07.001.

[13] Zheleva, I., Georgiev, I., Filipova, M., \& Menseidov, D. (2017, October). Mathematical modeling of the heat transfer during pyrolysis process used for end-of-life tires treatment. In AIP Conference Proceedings (Vol. 1895, No. 1, p. 030008). AIP Publishing LLC, https://doi.org/10.1063/1.5007367.

[14] Z. Zlatev, Computer treatment of large air pollution models, KLUWER Academic Publishers, Dorsrecht-Boston-London, 1995.

[15] Z. Zlatev, I. T. Dimov, Computational and Numerical Challenges in Environmental Modelling, Elsevier, Amsterdam, 2006. 


\section{Advances in Network Systems and Applications}

$\mathbf{T}$ HE rapid development of computer networks including wired and wireless networks observed today is very evolving, dynamic, and multidimensional. On the one hand, network technologies are used in virtually several areas that make human life easier and more comfortable. On the other hand, the rapid need for network deployment brings new challenges in network management and network design, which are reflected in hardware, software, services, and securityrelated problems. Every day, a new solution in the field of technology and applications of computer networks is released. The ANSA technical session is devoted to emphasizing upto-date topics in networking systems and technologies by covering problems and challenges related to the intensive multidimensional network developments. This session covers not only the technological side but also the societal and social impacts of network developments. The session is inclusive and spans a wide spectrum of networking-related topics.

The ANSA technical session is a great place to exchange ideas, conduct discussions, introduce new ideas and integrate scientists, practitioners, and scientific communities working in networking research themes.

\section{TOPICS}

- Networks architecture

- Networks management

- Quality-of-Service enhancement

- Performance modeling and analysis

- Fault-tolerant challenges and solutions

- 5G developments and applications

- Traffic identification and classification

- Switching and routing technologies

- Protocols design and implementation

- Wireless sensor networks

- Future Internet architectures

- Networked operating systems

- Industrial networks deployment

- Software-defined networks

- Self-organizing and self-healing networks
- Mulimedia in Computer Networks

- Communication quality and reliability

- Emerging aspects of networking systems

TeChnical Session Chairs

- Awad, Ali Ismail, Lule\&aring; University of Technology, Sweden

- Essai, Mohamed Hassan, AL-Azhar University, Egypt

- Furtak, Janusz, Military University of Technology

- Hodoň, Michal, University of Žilina, Slovakia

\section{Program COMMITTEe}

- Ajlouni, Naim, Istanbul Aydin University, Turkey

- Brzoza-Woch, Robert, AGH University of Science and Technology, Poland

- Chumachenko, Igor, Kharkiv National University of Municipal Economy named after Beketov, Ukraine

- Davidsson, Paul, Malmö University, Sweden

- Dotsenko, Sergii, Ukrainian State University of Railway Transport, Ukraine

- Długosz, Rafał, UTP University of Science and Technology, Bydgoszcz, Poland, Poland

- Elmougy, Samir, Mansoura University, Egypt

- Faria, Lincoln, Department of Computer Science, Fluminense Federal University, Brazil

- Khairova, Nina, National Technical University Kharkiv Polytechnic Institute, Ukraine

- Kochláň, Michal, University of Žilina, Slovakia

- Lavrov, Eugeniy, Sumy State University, Ukraine

- Salem, Abdel-Badeeh M., Ain Shams University, Egypt

- Smolarz, Andrzej, Lublin University of Technology, Poland

- Stamatescu, Grigore, University "Politehnica" of Bucharest, Romania

- Tymchuk, Sergiy, Kharkiv National Technical University of Agriculture. Petro Vasilenko, Ukraine

- Zieliński, Zbigniew, Military University of Technology 



\title{
Direct communication of edge elements in the Industrial Internet of Things
}

\author{
Piotr Hajder*, Mirosław Hajder ${ }^{\dagger}$, Mateusz Liput ${ }^{\dagger}$ and Mariusz Nycz ${ }^{\ddagger}$ \\ *AGH University of Science and Technology, Krakow, Poland, email: phajder@agh.edu.pl \\ ${ }^{\dagger}$ University of Information Technology and Management, Rzeszow, Poland \\ email: \{miroslaw.hajder,mateuszliput123\}@gmail.com \\ ${ }^{\ddagger}$ Rzeszow University of Technology, Rzeszow, Poland, email: mnycz@prz.edu.pl
}

\begin{abstract}
In this paper the results concerning changes introduced into the architecture of connections of an industrial computer network built on the basis of solutions characteristic of the Industrial Internet of Things were described. We introduce the new solutions improving such properties of industrial networks as: reliability, information security and usage of computational resources available in edge elements. The basic goal of the research was ensuring continuous functioning of the industrial computer network. Isolation of critical elements of the technological process can have far-reaching consequences, including local threats to workers' health and life, and even global technological disasters. Continuity of operation as a consequence of the reliability of the components of an information system is one of its most desirable properties.

The offered solution is based on the use of multi-channel reconfigured optical bus that is activated when the basic communication channels fail. In addition, the use of the described solutions for the build of a parallel, heterogeneous computing system based on elements of the Arduino and Raspberry Pi and system servers has been described and evaluated.
\end{abstract}

\section{INTRODUCTION}

$\mathbf{T}$ HE paper presents the results in the area of improving the effectiveness of Industrial Internet of Things (IIoT) used in the industrial information system (IIS). Presented results are based on a real system from medium and large industrial enterprises of the metallurgical, electromechanical and aviation industries.

During the research, our attention focused on improving three key IIoT properties for the enterprise: reliability along with derived characteristics, information security, and insufficiency of computational and memory resources. Information security is a guarantee of eliminating IIS unsanctioned access, use, disclosure, deformation, modification, investigation, recording or destruction of information [1], [2], [3], [4].

Reliability is a property of technical objects to maintain over time, within set limits, the values of all parameters necessary to perform the required functions in specific modes and conditions of use. The concept of dependability is associated with a number of other often cited system and network characteristics. Most often they are: fault tolerance; reliability; survivability, whose terms can be found in the literature [5], [6], [7]. They all indirectly determine the degree of continuity of IIS, key characteristics of IIS [8], [9]. Starting from the

The work was realized as a part of fundamental research financed by the Ministry of Science and Higher Education, grant no. 16.16.110.663 - task 18 definition presented earlier, information security can be a measure of its insensitivity to cyber criminals' attacks, which may also result in a loss of acting continuity. Although IIS is not an attractive object for traditional cyber criminals, such attacks performed for political reasons will occur more often and their negative consequences will increase. The solutions proposed in work allow improve the level of security in different way than currently used. In critical situations, IIS should have at its disposal minimal, attack-resistant processing resources that allow it to safely monitor its operations in critical situations, or terminate technical systems that threaten security. Starting the research, it was assumed that IIS will be build on the basis of industrial Internet of Things, which edge elements was built on the basis of Arduino or Raspberry platform [1], [10]. Thus, these elements can be used to build a heterogeneous, parallel processing system, largely insensitive to external attacks. The presented work consists of three sections. Section II presents the essence of industrial information systems using IIoT, based on the available literature, special features were distinguished, and potential threats of this type of systems were discussed. Section III discusses the proposed solution for connecting IIS edge elements, stressing the benefits and potential dangers. Section IV describes a research experiment whose aim was to confirm the effectiveness of the proposed solutions. In the Summary the analysis of the results of theoretical and empirical research is presented and discusses the current and future uses of the designed architecture. The work ends with the bibliography containing the works of other authors as well as the authors' own publications.

\section{INDUSTRIAL COMPUTER NETWORKS WITH IOT}

\section{A. Evolution of industrial computer networks}

In order to illustrate the evolution of interoperability of industrial network components, Fig. 1 shows its hierarchical model. The classic model presented there consist of the 4 layers, which the highest two use traditional communication channels based on Ethernet technology. Changes massively appear at the bottom-up automation level, at which interstitial communication takes place using industrial communication standards. We currently know over 50 types of industrial networks and data transmission protocols covered by the standard Fieldbus term [2], [11], [12]. Among of them the most known are: ProfiNet, HART, Modbus, Profibus, DeviceNet, CAN, 


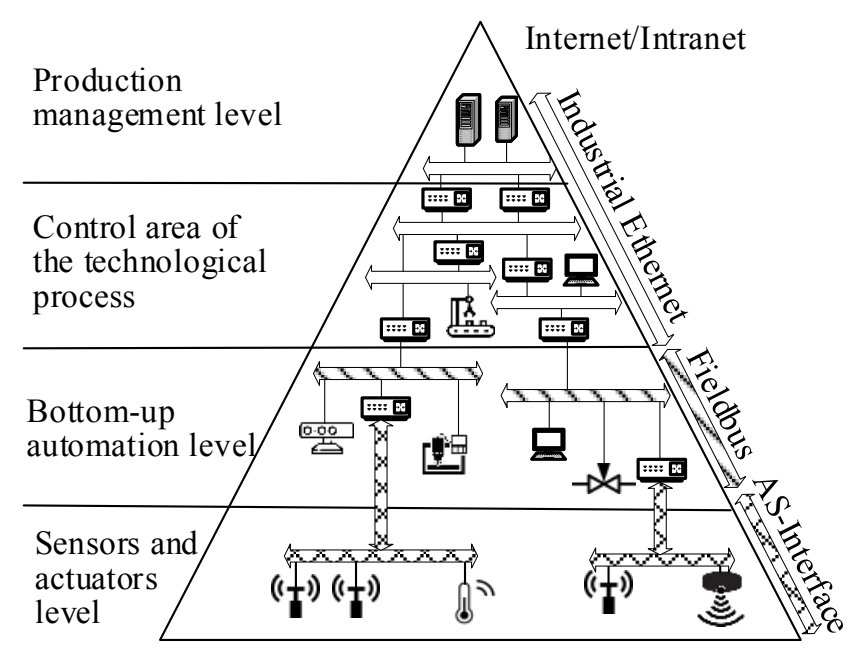

Fig. 1. Hierarchical model of an industrial computer network

CANopen, Lon-Works, FoxCom, ControlNet, SDS, Seriplex, BACnet, FIP, ASI, Industrial Ether-net, Wordfip, Foundation Fieldbus, Inter-bus, BitBus and others [2], [11]. Only some of them are widely used. Although a special FDT (Field Device Tool) interface has been designed to ensure interoperability of devices, technologies, data transmission protocols of different standards, their versions, generations and manufacturers, the need for communication between various system components remains a problem. Changes in the manner of communicating elements of the industrial computer network should first cover the bottom-up automation level and concern the use of methods and means of communication characteristic of the IoT network in this layer. However, the deepest modifications will include sensors and actuators level, in which, in place of passive measuring sensors, IoT compliant devices will be used, with a wide range of communication options. One of the most significant disadvantages of industrial computer networks operating in accordance with the architecture of Fig. 1 are the difficulties in communication controlling at sensors and actuators level. By equipping the lowest level of the model with intelligent measuring sensors, based on IIoT solutions, this defect will be removed and the control will be carried out using sensors based on the Arduino and Raspberry platforms. The use of hardware and software traffic analyzers located in all network segments at risk of attacks, both with wired and wireless communication is the first stage of the proposed changes in the architecture of the industrial computer network in Fig 1. For security reasons, sensors should not be connected to any of the automation, electronics or industrial metrology components used in the system. Thus, the operation of the monitoring subsystem in any negative way does not translate into the operation of the enterprise's integrated information service system. Unfortunately, in a number of cases this solution can be troublesome or even impossible to implement. An acceptable solution is the use of a further reconfigured connection network. For better illustration

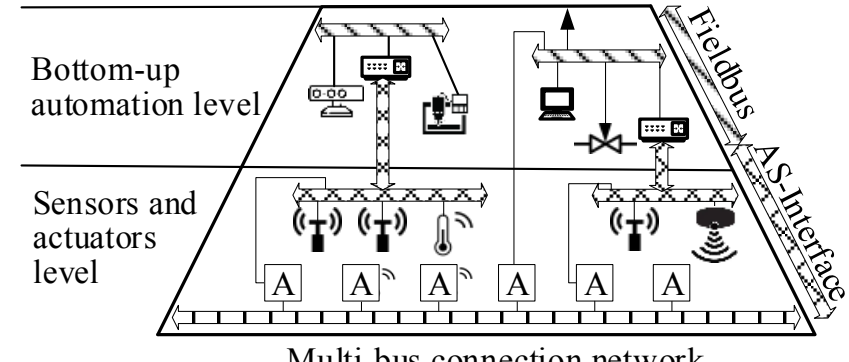

Fig. 2. Hierarchical model of the industrial network. A - software and hardware network analyzer

of the changes made in the traditional industrial network architecture, the bottom two layers of the new network are presented in Fig. 2. Additional software and hardware traffic analyzers $A$ have been added to sensors and actuators level. They were made using Raspberry $\mathrm{Pi}$ or Arduino platforms equipped with software and additional communication interfaces. The main goal for analyzers is to detect traffic anomalies in tracked network segments. Depending on the interface, wired or wireless network segments can be analyzed using the following protocols: Ethernet, Fieldbus AS-Interface, both on sensors and actuators and bottom-up automation levels. The proposed solution based on autonomous traffic analyzers, besides its advantages (significant improvement of information security and availability of computational resources), in small installations is characterized by high implementation costs. In large enterprise networks, these costs (around 250 euro per analyzer) are negligible. Therefore, as a part of the research, the traffic analysis technology based on IIoT components, for which traffic monitoring is not their main function, has been positively verified. The introduction to the lowest level of the industrial network model of software and hardware traffic analyzers is the first of the modifications made in the classic industrial computer network. The second change applied involves the implementation of an additional multi-bus communication network used exclusively by traffic analyzers. Separating the bus from the rest of the network ensures its insensitivity to possible attacks. The bus is a communication element that can be used in critical moments, e.g. for emergency securing of technological operations. From the point of view of modern graph theory [13], [14], [15], [16], [17] and hierarchical systems [18], [19], the modified network is a hierarchical star whose leaves have been connected by means of an additional horizontal communication channel.

\section{B. Methods for describing bus systems}

The most important feature of the communication bus (Fig. 2) is the possibility of distributed reconfiguration. This means that the selection of a specific communication channel will be made directly by traffic analyzers $A$. The organization of connections can be selected so that the edge communication parameters imposed by the designer, such as: maximum transmission delay, integration of only indicated network nodes, 
network life at the indicated level will be met at any time. This requires, among others mathematical description of bus network connections. Mathematical description of bus systems is presented in [9], [20], [21]. In order to improvements in the machine analysis and synthesis of the bus connection network, an algebraic topology notation based on an algebra of connected finite non-directed graphs has been proposed. Graph algebra is defined in the Definition 1.

Definition 1. The pair $A=(D, \Omega)$ will be called the universal graph algebra over the universe $U$, if $D$ is a set of graphs with vertices from the set $U$, and the signature $\Omega$ enables the zero operation $\Lambda$, binary operations of adding a vertex, adding an edge, removing a vertex and removing edges.

First, let's define the theorem, which can be used to describe the topology of selected networks.

Theorem 1. The minimal elements of the $A G=(A, \Omega)$ algebra will be trees of the form $G_{u}^{0}$, where: $G_{u}^{0}=(V=$ $\{u\}, E=\emptyset)$ - an empty tree composed of the vertex $u$.

Using the Theorem 1 we will analyze the trees:

1) $T_{u, v}=(V=\{u, v\}, E=\{(u, v)\})$;

2) $T_{u, v}^{w}=(V=\{u, v, w\}, E=\{(u, w),(w, v)\})$.

Using algebra, the above trees can be represented by the minimum elements:

1) $T_{u, v}=(V=\{u, v\}, E=\{(u, v)\})=w_{i k}\left(G_{u}^{0} \cup\right.$ $\left.G_{v}^{0}, u, v\right)=T_{u, v}=G_{u}^{0} * G_{v}^{0}=G_{u}^{0} *{ }_{f} G_{v}^{0}(f(u)=v)$;

2) $T_{u, v}^{w}=(V=\{u, w, v\}, E=\{(u, w),(w, v)\})=T_{u, w} \cup$ $T_{w, v}=w_{i k}\left(T_{u}^{0} \cup T_{w}^{0}, u, w\right) \cup w_{i k}\left(T_{w}^{0} \cup T_{v}^{0}, w, v\right)=$ $\left(T_{u}^{0} * T_{w}^{0}\right) \cup\left(T_{v}^{0} * T_{w}^{0}\right)$.

where: $w_{i k}$ - the operation of adding an edge into connected graph; $*$ - the operation of combining two connected graphs. In addition, the result of combining two graphs will be a connected graph, if even one or two of them does not meet connectivity condition. In particular, for a connected graph $G$, graphs corresponding to the expressions $\left(T_{u}^{0} \cup T_{v}^{0}\right) * G$ and $\left(T_{u}^{0} \cup T_{v}^{0}\right) *\left(T_{u}^{0} \cup T_{v}^{0}\right) *\left(T_{w}^{0} \cup T_{s}^{0}\right)$, where: $u, v, w, s$ in pairs different vertices, will be connected graphs. The algebraic expression describing the single channel bus in graph algebra notation has the following form: $\left(T_{S_{1}}^{0} \cup \ldots \cup T_{S_{r}}^{0} \cup\right.$ $\left.T_{K_{1}}^{0} \cup \ldots T_{K_{n}}^{0}\right) * T_{B}^{0}$. Technical solutions developed on the basis of research are based solely on the multi-channel bus. Suppose the complete multi-bus (i.e. each service provider and recipient is connected to each of the buses) consists of $m$ channels, $S_{r}$ recipients and $K_{n}$ service providers. Then its physical form and its notation in the form of a tripartite graph have the form presented in Fig. 3. It can be assumed from a technical point of view, that the $B_{1}, \ldots, B_{m}$ buses are logical channels functioning in the one common physical channel. The algebraic expression describing the above network has the following form:

$$
\left(T_{S_{1}}^{0} \cup \ldots \cup T_{S_{r}}^{0} \cup T_{k_{1}}^{0} \cup \ldots \cup T_{k_{n}}^{0}\right) * T_{B}^{0}
$$

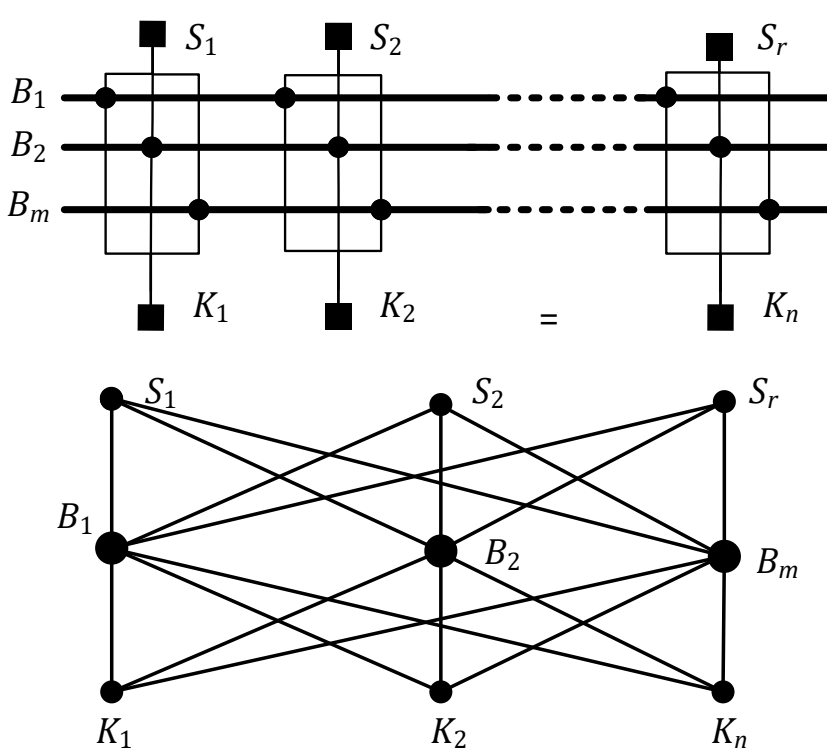

Fig. 3. The network with multi-channel bus presented as tripartite graph

\section{Additional functions of modified network}

Having a set of independent computational nodes and reconfigured connections in one system creates an excellent opportunity to construct a scalable computational unit. Particular interest in this type of solutions in IIS result from the following conditions:

1) The concentration of cyber-attacks on the top two layers of the network model. They have the resources necessary to safely support production technologies. Attacks on the lower layers of the model are very rare;

2) In order to maximize the level of security it is appropriate to separate computational diagnostic components outside the areas of the industrial network model available to cybercriminals.

In the case of the cyber-attack on IIS resources, degradation of computational and communication resources used in the security subsystem occurs. If the threat detection system is based solely on polynomial combinatorial algorithms or the analysis of limited data, the problem of insufficient computational resources rather does not appear. It becomes valid at the time of application of time-complex algorithms, e.g. providing machine learning or bio-inspired analysis. If the detection system acts only as a part of the top layer, degradation of its resources is highly possible. That is why, in some enterprises, independent computational resources with low usage in a stable mode of operation are constructed on the basis of the edge network components, designed solely to operate the detection system. Their load increases drastically when threats appear. The solution offered assumes the separation of scalable computational resources, independent of other components of the upper IIS layer. For this purpose, it was decided to prioritize the procedures for parallelizing processing in a heterogeneous environment with multi-channel optical connections. Communication is based on the repeatedly 


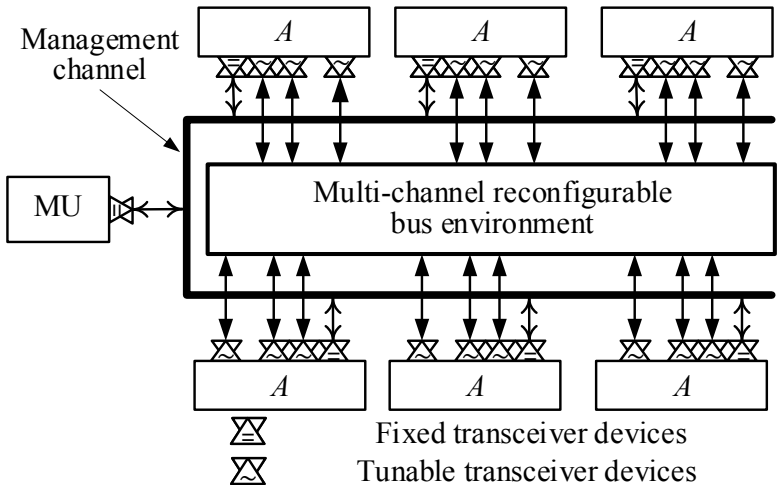

Fig. 4. The concept of the system: MU - management node, controlling bus system

folded bus with the possibility of its physical division and virtual reconfiguration of the connection network. The first level of parallelization is based on a separate security sever and uses CUDA technology [22], [23]. At the second level, in the event of a computational power deficit, a heterogeneous computational system is dynamically constructed using the available resources of analyzers based on dedicated hardware of IIoT components.

\section{Bus communication systems in IIS}

The basic concept of a parallel reconfigurable computational system with multi-bus connections is shown in Fig. 4. It was assumed that all computational functions will be performed in the system based on the resources of the management node $M U$ and $A$ traffic analyzers. For security reasons, the computational servers of the upper layers are not used. Each of the analyzers has been equipped with at least one fixed and one variable single-channel communication interface. If it is possible, then the parameters of each of the fixed channels are unique in the entire structure, thanks to which there is no limit to setting up networks with any connection architecture. A deviation from this rule is the management channel to which all computational nodes are connected. This channels always uses broadcast and is only intended for sending information about the configuration of transceivers. The use of a device that supports a fixed communication channel is also expedient from an economic point of view - the price of these devices is many times lower than tunable devices. Each of the $A$ analyzers is equipped with a set (2 to 4 ) of fixed or tunable transceiver devices. They are connected in various ways to logical buses (e.g. wave channels) $B_{1}, \ldots, B_{4}$, thus responding to different user needs. The use of independent optical fibers (physical buses) instead of logical buses significantly changes the properties of the above organizations. If the system from Fig. 5a is equipped with integrated transceivers and logical buses $B_{1}, \ldots, B_{4}$ it can be used to improve the failure resistance of transceivers. In addition, if many analyzers compete for access to logical buses, the amount of information provided or retrieved from the node may be multiplied by using routing addressed to this type of architecture. If $B_{1}, B_{2}$ buses are physical channels, the
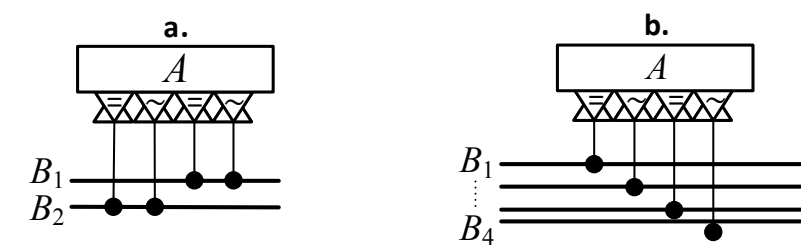

c.

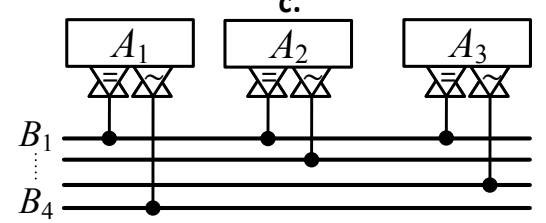

Fig. 5. Connections between node and the virtual bus: a. multiple complete; b. single complete; c. partial

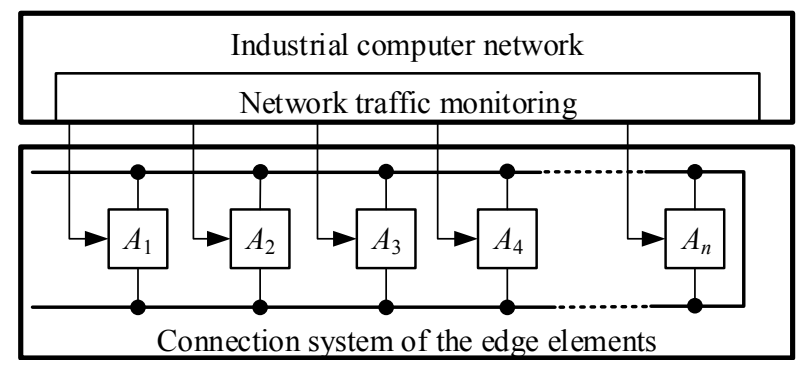

Fig. 6. Functional components of the industrial computer network with horizontal connection of edge elements

system from Fig. 5a improves fault tolerance of this type of channel. Past experience shows that damage to communication channels usually occur at the physical level, i.e. optical fibers or transceivers. Similarly, the other architectures from Fig. 5 can be analyzed. All the analyzers operate at sensors and actuators level. If the transmitting and receiving devices can be separated, and instead of the traditional bus the folded bus is used, the following effective methods of minimizing communication delays appear, by selecting the appropriate part of the bus, where the information signal is delivered or supplied. Each of the analyzers has its own computational power, which can be used in the system, both in a group and independently for each device.Computational servers will be made available in the system to increase computational efficiency. They can be independent devices or components of an industrial computer network deployed at the production management level.

\section{CONNECTION ARCHITECTURE OF THE EDGE ELEMENTS}

\section{A. The concept of the system organization}

Let's analyze a fragment of the architecture of an industrial computer network using IIoT with horizontal connection of edge elements based on multi-bus communication with passive joining of users. The functional components of such a system are shown in Fig. 6. Further considerations will apply only to the edge connection system located in the lower part of 


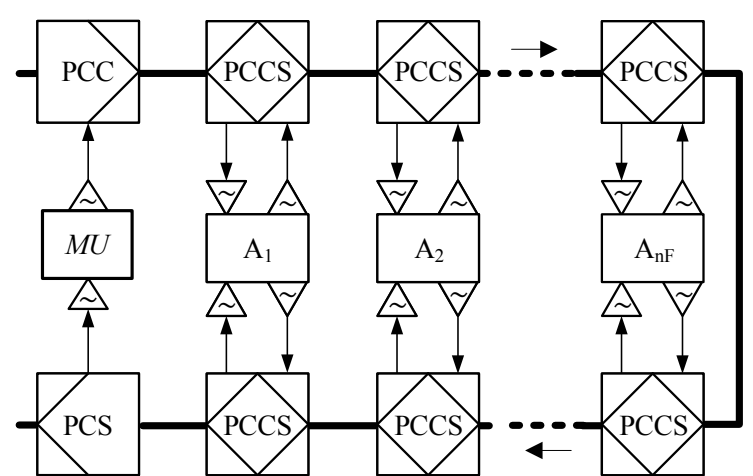

Fig. 7. Connection of edge elements by means of a multi-channel simplex folded bus with tunable transceiver elements with a symmetrical organization without load balancing

Fig. 6. Each of the $n$ analyzers is connected to the lower and upper part of the folded multi-channel bus. Both statements are used only to perform communication operations as a part of a computational system built based on edge elements. The number of connections is limited only by the possibilities of the edge element. In addition, each of the analyzers receives a signal for analysis from the industrial computer network to fulfill its basic functions. The power of edge elements (e.g. based on Raspberry Pi 4) is so high that they can fulfill the function of analyzers and computational elements solving other tasks. In Fig. 6, for a known reason, the management bus and the server controlling the bus set are omitted. There are many alternative ways to improve the communication efficiency of the multi-bus systems. The range of possible methods and measures to improve the quality of inter-node connections in multi-channel industrial networks is very wide. However, not all available solutions are equally attractive. Since the priority of the work being performed is to minimize the costs of IIS construction and operation, it was decided to use a passive bus in which the organization of logical communication channels is performed by means of transceivers directly in the connected node. For similar reasons, it was decided to use simplex bus channels. Fault tolerance, reliability and survivability considerations have decided to use multiple folded physical channels. Their use is particularly beneficial from the point of view of the availability of the communication subsystem. The networks based on them maintain connectivity even with repeated damage to the physical network. For similar reasons, it was decided to use symmetrical communication channels without load balancing. Passive connection architecture plays a decisive role in organizing the connections of edge elements. Other choices are of secondary importance. The connection system architecture meeting the above criteria is shown in Fig. 7. Each of the analyzers (computational nodes) has been equipped with two tunable transmitters and two tunable receivers synchronized with them in pairs. Unlike previously discussed solutions, the management node was also equipped with tunable transceiver devices. The application of this solution resulted from the desire to unify the system. a.

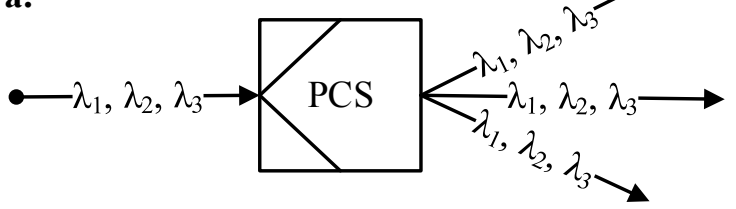

b.

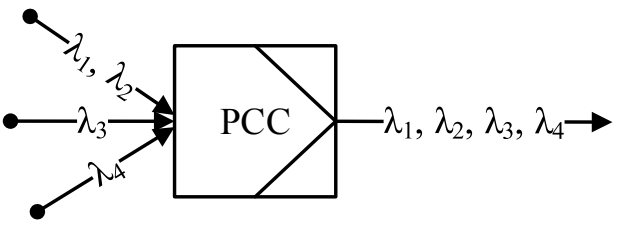

c.

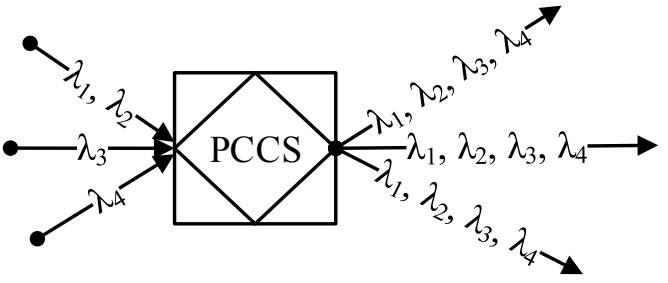

Fig. 8. Passive couplers: a. Physical channel separator (PCS); b. Physical channel connector (PCC); c. Physical channel connector-splitter (PCCS)

In practice, we also use other solutions to organize the management channel, including: a pre-imposed logical channel with a fixed transceiver and receiver, and the organization of the management channel in an independent physical channel. The communication components of the system in Fig. 7 are shown in Fig. 8. As $\lambda$ we denote independent communication channels. All of the following elements are passive and their purchase does not generate high costs. They are available on the market.

\section{B. Determining acceptable parameters of the system}

Let's estimate acceptable values of the system parameters shown in Fig. 8. The minimum number of logical transmission channels necessary to build a connected communication system is 2 . The first of the channels is used as a management, the second connects computational nodes (analyzers - network edge nodes). Both channels operate in broadcast mode. The maximum number of $K_{\max }^{l k}$ required to build a complete topology with direct duplex connections is:

$$
K_{\text {max }}^{l k}=n_{F}^{2}-n_{F}+1
$$

where: $n_{F}$ - number of computational nodes of the system. Minimum number of receiving and transmitting devices of the $i$-th node of connected system, $k_{i_{\text {min }}}^{R}$ and $k_{i_{m i n}}^{T}$ respectively, are identical and equals $2\left(k_{i m i n}^{R}=k_{i_{m i n}}^{T}=2\right)$. Maximum number of $k_{i m i n}^{R}$ and $k_{i_{m i n}}^{T}$ are also even and equal $n_{F}$. The total minimum numbers of receiving and transmitting devices, $K_{\min }^{R}$ and $K_{\min }^{T}$ respectively, are defined as:

$$
\begin{aligned}
& K_{\text {min }}^{R}=\sum_{i=1}^{n_{F}} k_{i_{\text {min }}}^{R}+1=2 n_{F}+1 \\
& K_{\text {min }}^{T}=\sum_{i=1}^{n_{F}} k_{i_{\text {min }}}^{T}+1=2 n_{F}+1
\end{aligned}
$$


Let's estimate system delays without routing. The value of the maximum transmission delay $\tau_{\max }$ depends not only on the technical characteristics (e.g. geographical size) of the system, but also on the occupancy level of the transceiver and communication bus. Let's consider a simple example: nodes $A_{1}$ and $A_{2}$ equipped with sets of transceiver elements will communicate. If any upper receiving element $A_{2}$ is free, the distance over which the signal will be transmitted is minimal (part of the upper bus fragment) and the number of passive splitting elements passed is 2 . However, if the upper receivers of node $A_{2}$ are occupied, the information signal will only be received by the lower element $A_{2}$. The signal will cover almost the entire bus length, including $2 n_{F}-1$ passive PCCS components. This should not happen if the system provides for optimization of transmission performed by the management node. The system's answer will be blocking the communication channel. Determining the maximum delay value will start from the wort case, when directions of the physical and logical channels are opposite and the length of the communication channel will be maximum. Let there be unloaded logical channels and unoccupied transceiver devices in the analyzed system. The maximum delay will occur on the path between nodes $A_{2}$ and $A_{1}$ because the information channel will be created between the upper interface $A_{2}$ and the lower $A_{1}$. In systems without routing, the described situation is acceptable and in no way results from the occupation of any receiving or transmitting elements. In the case of a homogeneous connection system, in which the distance between the computational nodes and the time parameters of the components are identical, the delay value $\tau_{\max }$ is described by the following expression:

$$
\tau_{\max }=t^{B_{2 \rightarrow 1}}+2\left(n_{F}-1\right) t^{P C C S}+t_{\nu}^{R}+t_{\nu}^{T}
$$

where: $t^{B_{2 \rightarrow 1}}$ - transmission delay of the physical channel between $A_{2}$ (device is connected to the upper part of the bus) and $A_{1}$ (device is integrated with the lower part of the bus); $t^{P C C S}$ - delay of the integrating PCCS component; $t_{\nu}^{R}, t_{\nu}^{T}$ time delay of receiving and transmitting devices, respectively. In the most unfavorable case, the length of the used physical channel fragment is comparable to its total length. Therefore, $t^{B_{2 \rightarrow 1}}$, where $t^{B}$ - signal transmission delay between bus ends. In addition, for large $n_{F}$ values, the delays of the transceiver devices can be neglected. Then, expression (4) can be written in a simplified form:

$$
\tau_{\max } \approx t^{B}+2 n_{F} t^{P C C S}
$$

We will determine the maximum delay in sending information through a channel in a routed communication network. We will use the previously proposed routing algorithm that transfers the creation of the transmission channel between the upper and lower parts of the folded bus. In a routed system, the maximum delay occurs when passing information over the channel connection $A_{1}$ to $A_{n_{F}}$ or $A_{n_{F}}$ with $A_{1}$. In this case, the expression (4) can be written as:

$$
\tau_{\max }^{r}=t^{B_{1 \rightarrow n_{F}}}+n_{F} t^{P C C S}+t_{\nu}^{R}+t_{\nu}^{T}
$$

where: $\tau_{\max }^{r}$ - maximal transmission delay in the architecture with routing; $t^{B_{1 \rightarrow n_{F}}}$ - signal transmission delay through the physical bus between nodes $A_{1}$ and $A_{n_{F}}$. If the upper and lower part of the folded bus are symmetric, then $t^{B_{1 \rightarrow n_{F}}} \approx 0.5 t^{B_{2 \rightarrow 1}}$. Considering the expressions (4) and (6), the following condition can be accepted:

$$
\tau_{\max }^{r}=0.5 \tau_{\max }
$$

In the computational systems with symmetric architecture of the transceiver devices, minimal value of the transmission delay does not depend on whether the system uses routing and for types (with or without routing) is determined by the expression:

$$
\tau_{\min }=\tau_{\min }^{r}=t^{B_{1 \rightarrow 2}}+2 t^{P C C S}+t_{\nu}^{R}+t_{\nu}^{T}
$$

where: $\tau_{\text {min }}^{r}$ - minimum value of the transmission delay for the architecture with routing; $t^{B_{1 \rightarrow 2}}$ - delay of the signal transmitting through the physical bus between neighboring nodes, in particular between $A_{1}$ and $A_{2}$. The last, significant time parameter of the computational system connection efficiency is the average value of the transmission delay $\tau_{\text {avg. }}$. We will specify its value for organizations without routing. If the distribution requests for the set of transmission channels is homogeneous, the value $\tau_{\text {avg }}$ can be defined as the arithmetic mean of the minimum and maximum delay of signal transmission through the channel, i.e.:

$$
\tau_{\text {avg }}=0.5\left(\tau_{\min }+\tau_{\max }\right)
$$

Considering the expressions (4) and (8), the formula (9) can be written in the following way:

$$
\begin{aligned}
& \tau_{\text {avg }}=0.5\left(t^{B_{2 \rightarrow 1}}+\left(2 n_{F}-1\right) t^{P C C S}+t_{\nu}^{R}+t_{\nu}^{T}+\right. \\
& +t^{B_{1 \rightarrow 2}}+2 t^{P C C S}+t_{\nu}^{R}+t_{\nu}^{T}= \\
& =0.5\left(t^{B_{2 \rightarrow 1}}+t^{B_{1 \rightarrow 2}}+\left(2 n_{F}+1\right) t^{P C C S}+2 t_{\nu}^{R}+2 t_{\nu}^{T}\right)
\end{aligned}
$$

Note that $t^{B_{2 \rightarrow 1}} \approx t^{B}$ and $t^{B_{1 \rightarrow 2}} \approx t^{B} / 2 n_{F}$. Therefore, if $n_{F} \gg 2$ the above expression can be written as follows:

$$
\tau_{\text {avg }} \approx 0.5 \tau_{\max }
$$

Based on the expression (9) we will determine the average delay $\tau_{\text {avg }}^{r}$ for an organization with routing. Using the expressions (6) and (8), delay value can be estimated:

$$
\begin{aligned}
& \tau_{\text {avg }}^{r}=0.5\left(t^{B_{1 \rightarrow n_{F}}}+n_{F} t^{P C C S}+t_{\nu}^{R}+t_{\nu}^{T}+t^{B_{1 \rightarrow 2}}+\right. \\
& \left.+2 t^{P C C S}+t_{\nu}^{R}+t_{\nu}^{T}\right)= \\
& =0.5\left(t^{B_{1 \rightarrow n_{F}}}+t^{B_{1 \rightarrow 2}}+\left(n_{F}+2\right) t^{P C C S}+2 t_{\nu}^{R}+2 t_{\nu}^{T}\right)
\end{aligned}
$$

If we assume that $t^{B_{1 \rightarrow n_{F}}} \approx 0.5 t^{B}$ and $t^{B_{1 \rightarrow 2}} \approx t^{B} / 2 n_{F}$, then for $n_{F} \gg 2, t^{B_{1 \rightarrow n_{F}}} \gg t^{B_{1 \rightarrow 2}}$. Then:

$$
\tau_{a v g} \approx 0.5 \tau_{\max }^{r} \approx 0.25 \tau_{\max }
$$

Analysis of transmission time characteristics shows that routing improves most of them. At the same time, along with the reduction in the length of the transmission channel, the signal attenuation also decreases, which minimizes the requirements, and thus reduces the overall cost of the system construction. The basic conclusion of the above analysis is the desirability of extensive use of the system with routing. 


\section{Description of the system functioning}

The device developed at the current stage is intended to improve the security of the industrial information system. Built-in autonomous analyzers record all manifestations of network activity. The analyzers also make a preliminary detection of any anomalies, this process is continued in the management server. Each of the analyzers can work in one of several modes. In basic mode, the analyzer tracks the traffic in the assigned wired or wireless network segment. All analyzers are connected via two communication channels. The first of them - broadcasting - controls communication between the analyzers and the management server, while the second (direct - dynamically set up) is the right channel for transferring information between security system objects. The acquired data on traffic anomalies in the segments are stored in the monitoring unit (edge element) and periodically sent to the server(s) of the security system. For this purpose, a two-point virtual channel connecting the selected monitoring node with the appropriate server is dynamically established. The management channel participates in the connection setup procedure. Dynamic set up of the transmission channels is also beneficial from a security point of view. In this way, the number of entries to the system available to the intruder is minimized. The number of virtual channels operating simultaneously depends only on the number of transceivers installed in the servers. It is allowed to connect all analyzers and servers with a set of broadcast channels. Pre-processing of traffic information in the segment is performed directly in the analyzers. For this purpose, each monitoring node stores the symptoms of threats, which are in fact traffic patterns, indicating the appearance of a threat. The list of symptoms is not constant, it is created autonomously by computational servers based on data sent by nodes using a set of analytical algorithms using AI methods (machine learning, big data algorithms) and biologically motivated tools (immunological and evolutionary methods). Patterns are periodically sent to monitoring nodes. If traffic similar to one of the patterns appears in the segment, the monitoring node requests to set up a temporary two-point communication channel connecting it with the selected computational server. The channel set-up consists in determining the number of the common communication channel for the monitoring node and the server. Until the dangerous symptom stops, the traffic is analyzed in real time on the security server. Further decisions are made about how to deal with the potential threat. According to Fig. 5, the server can simultaneously support multiple virtual networks, it can also process information from multiple analyzers. Thanks to this, the security system can work in the real time. If the detected anomalies in the assessment of the security server may threaten the security of IIS, the functioning of the system is limited and in extreme cases the system is stopped. The algorithm of functioning of the threat detection system is presented in Fig. 9. The described procedure illustrates one of many possible works performed by the edge elements thanks to their connection via a bus communication channel. With

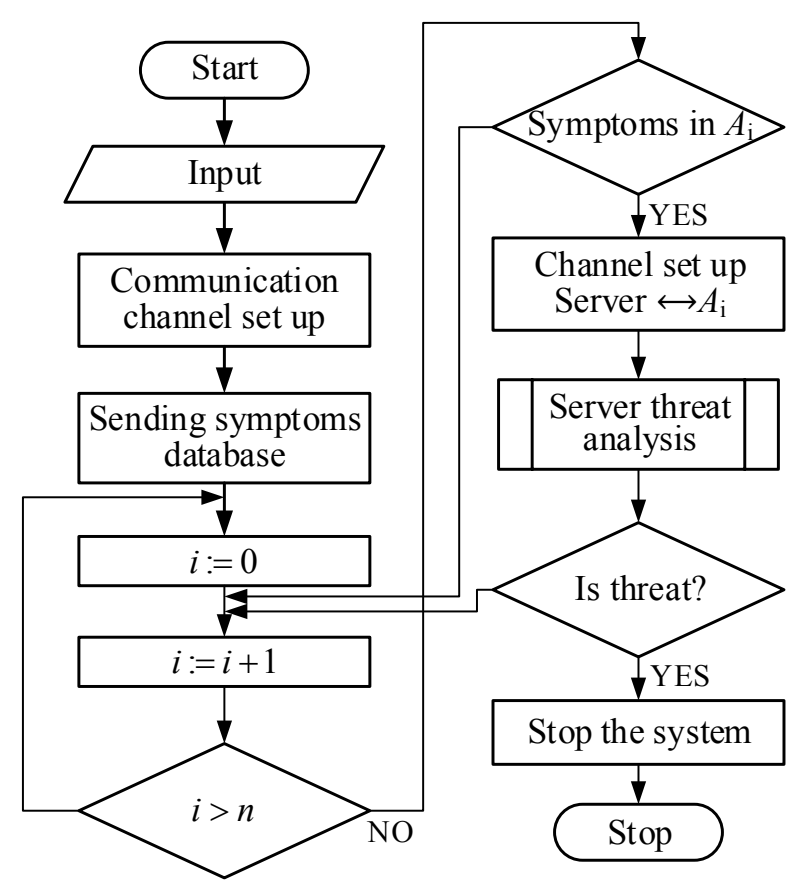

Fig. 9. Stages of functioning of the system of connecting edge elements

time, as the edge element resources increase, the range of such activities will be practically unlimited.

\section{EXPERIMENTAL RESULTS}

In order to process results from the research, we used IBM SPSS Modeller, OriginPro, Process Explorer and softwarehardware prototype of designed device. It consisted of two steps. The first of them performed the procedure of filling the symptom base with attack patterns. Filling was outlined until balanced accuracy on the training and test set exceeded 0.986. During the process, normalization of numeric parameter values and conversion to binary values of text parameters, as well as classification of database records, was performed. In the second step, using the software testing sequence generator imitating the output of the measurement sensors and the machine time measurement tool, the attack detection time was determined for the given accuracy and completeness of attack detection. The minimum value of all basic detection parameters (correct classification, precision, completeness and metric) was set at a minimum of $80 \%$. Teardrop, smurf, satan, portsweep, pod, normal,nmap neptune, ipsweep and back attacks were examined. The study analyzed the effectiveness of four architectures: CPU, CPU + $8 \mathrm{RPi}, \mathrm{CPU}+\mathrm{GPU}$ and $\mathrm{CPU}+\mathrm{GPU}+8 \mathrm{RPi}$. Each of the experiments was performed 1000 times, the published results were subjected to processing characteristics of empirical data. The results of the experiment are shown in Fig. 10. Previous work has shown the desirability of using edge elements to increase the computational power available in industrial computer networks. With relatively small investments, it can get performance comparable with many times more expensive commercial solutions. Currently, 


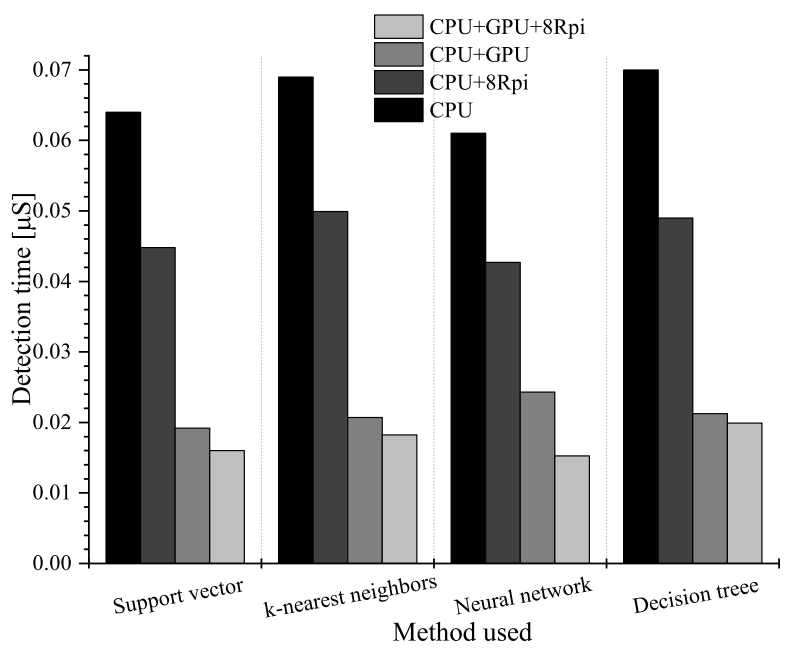

Fig. 10. Attack detection times for various computational system configurations and detection methods

the designed solution in real conditions is being tested, in which threats will be detected. Previous work with the test system utilized traffic load and computational tasks from a test event generator configured to bring tasks and loads similar to those found in an industrial computer network.

\section{Summary AND FURTHER WORKS}

The paper presented the method of horizontal joining of edge elements of an industrial computer network. Due to the wide application of passive transmission network based on folded buses, the solution is characterized by low costs and high resistance to physical channels damage. The solution has a very wide development potential, resulting, among others with:

1) Possibilities of repeated folding of the communication bus. If the number of interfaces allowing to build transceivers increases, it will be possible to effectively use folding, increasing the system's resilience and minimizing communication delays due to routing;

2) Possibilities of splitting or grouping buses. Users can also be grouped [9]. Thanks to this, there are wide possibilities of organizing network traffic ensuring load balancing of communication channels and minimizing delays;

3) Continuous development of the system's hardware base. Arduino and Raspberry devices are constantly evolving, providing new technical possibilities. First of all, improving the hardware base will result in an increase in the number of independent communication channels connecting the processing element to the bus system.

Further work will focus on increasing the size of the prototype and developing tools for computer-aided design of such sys- tems. The functionalities offered will be combined with edge elements, currently focused on the analysis of information security threats monitored by industrial computer network analyzers. Currently available computational resources are used to detect threats based on collected traffic information. A number of modern methods are used for this purpose, including machine learning and intelligent data analysis. In the future, it is planned to develop methods to support the resources of industrial computer networks in solving time-consuming tasks, such as combinatorial optimization and graph algorithms.

\section{REFERENCES}

[1] A. Dehghantanga and K. K. R. Choo, Handbook of Big Data and IoT Security. Springer, 2019.

[2] C. Alcaraz, Security and Privacy Trends in the Industrial Internet of Things. Springer, 2019.

[3] M. Alazab and M. J. Tang, Deep Learning Applications for Cyber Security. Springer Nature, 2019.

[4] M. Collins, Network Security Through Data Analysis. Building Situational Awareness. Sebastopol: O'Reilly, 2013.

[5] M. J. Zuo, Optimal reliability modeling: principles and applications. Hoboken: John Wiley \& Sons, 2003.

[6] M. L. Shooman, Reliability of Computer Systems and Networks: Fault Tolerance Analysis and Design. New York: John Wiley \& Sons, 2002.

[7] P. Stavroulakis, Reliability, survivability and quality of large scale telecommunication systems. Chichester: John Wiley \& Sons, 2003.

[8] S. Bhattacharjee, Practical Industrial Internet of Things Security. Birmingham: Packt, 2018.

[9] P. Hajder and Łukasz Rauch, "Reconfiguration of the multi-channel communication system with hierarchical structure and distributed passive switching," in Computational Science - ICCS 2019, $19^{\text {th }}$ International Conference. Faro, Portugal: Springer, 2019, pp. 502-516.

[10] A. Banafa, Secure and Smart Internet of Things (IoT). Using Blockchain and AI. Gistrup: River Publishers, 2018.

[11] A. Kott and E. J. Colbert, Cyber-security of SCADA and Other Industrial Control Systems. Cham: Springer Nature, 2016.

[12] F. Paganelli and G. J. van Viet, Resilience and Reliability on AWS Sebastopol: O'Reilly Media, 2013.

[13] B. Bollobas, Modern Graph Theory. New York: Springer, 1998.

[14] J. Bang-Jensen and G. Z. Gutin, Digraphs: Theory, Algorithms and Applications. London: Springer, 2010.

[15] M. D. Rahman, Basic Graph Theory. Cham: Springer International Publishing AG, 2017.

[16] P. Mathis, Graphs and Networks. New York: Wiley \& Sons, 2010.

[17] R. Diestel, Graph Theory. New York: Springer, 2010.

[18] N. J. Smith and A. P. Sage, "An introduction to hierarchical systems theory," Computers \& Electrical Engineering, vol. 1, pp. 55-71, 1973.

[19] M. D. Mesarovic, D. Macko, and Y. Takahara, Theory of hierarchical multilevel systems. New York: Academic Press, 1970.

[20] M. Hajder and J. Kolbusz, Formalizacja projektowania architektury systemów informacyjnych. Rzeszow: Wyższa Szkoła Informatyki i Zarządzania, 2015

[21] M. Hajder, M. Nycz, and J. Kolbusz, "Grafowe reprezentacje obiektów technicznych," in Innowacyjna gmina. Informatyka $w$ jednostkach samorząu terytorialnego, M. Hajder, Ed. Rzeszów: Wyższa Szkoła Informatyki i Zarządzania, 2014, pp. 163-172.

[22] L. Lladis, Y. Kim, S. sarafijanovic, and V. Sarafijanovic, "Performance evaluation of a tape library system," in IEEE $24^{\text {th }}$ International Symposium on Modeling, Analysis and Simulation of Computer and Telecommunication Systems (MASCOTS), London, 2016.

[23] T. Sterling, M. Anderson, and M. Brodowicz, High Performance Computing. Modern Systems and Practices. Cambridge, MA, USA: Morgan Kaufmann, 2018. 


\section{$1^{\text {st }}$ International Forum on Cyber Security, Privacy and Trust}

$\mathbf{N}$ OWADAYS, information security works as a backbone for protecting both user data and electronic transactions. Protecting communications and data infrastructures of an increasingly inter-connected world have become vital nowadays. Security has emerged as an important scientific discipline whose many multifaceted complexities deserve the attention and synergy of computer science, engineering, and information systems communities. Information security has some wellfounded technical research directions which encompass access level (user authentication and authorization), protocol security, software security, and data cryptography. Moreover, some other emerging topics related to organizational security aspects have appeared beyond the long-standing research directions.

The International Forum of Cyber Security, Privacy, and Trust (NEMESIS'20) as a successor of International Conference on Cyber Security, Privacy, and Trust (INSERT'19) focuses on the diversity of the cyber information security developments and deployments in order to highlight the most recent challenges and report the most recent researches. The session is an umbrella for all cyber security technical aspects, user privacy techniques, and trust. In addition, it goes beyond the technicalities and covers some emerging topics like social and organizational security research directions. NEMESIS'20 serves as a forum of presentation of theoretical, applied research papers, case studies, implementation experiences as well as work-in-progress results in cyber security. NEMESIS'20 is intended to attract researchers and practitioners from academia and industry and provides an international discussion forum in order to share their experiences and their ideas concerning emerging aspects in information security met in different application domains. This opens doors for highlighting unknown research directions and tackling modern research challenges. The objectives of the NEMESIS'20 can be summarized as follows:

- To review and conclude research findings in cyber security and other security domains, focused on the protection of different kinds of assets and processes, and to identify approaches that may be useful in the application domains of information security.

- To find synergy between different approaches, allowing elaborating integrated security solutions, e.g. integrate different risk-based management systems.

- To exchange security-related knowledge and experience between experts to improve existing methods and tools and adopt them to new application areas

\section{TOPICS}

- Biometric technologies

- Cryptography and cryptanalysis

- Critical infrastructure protection

- Security of wireless sensor networks

- Hardware-oriented information security

- Organization- related information security

- Social engineering and human aspects in cyber security

- Individuals identification and privacy protection methods

- Pedagogical approaches for information security education

- Information security and business continuity management

- Tools supporting security management and development

- Decision support systems for information security

- Trust in emerging technologies and applications

- Digital right management and data protection

- Threats and countermeasures for cybercrimes

- Ethical challenges in user privacy and trust

- Cyber and physical security infrastructures

- Risk assessment and management

- Steganography and watermarking

- Digital forensics and crime science

- Security knowledge management

- Security of cyber-physical systems

- Privacy enhancing technologies

- Trust and reputation models

- Misuse and intrusion detection

- Data hide and watermarking

- Cloud and big data security

- Computer network security

- Assurance methods

- Security statistics

\section{TECHNiCAL SESSION CHAIRS}

- Awad, Ali Ismail, Luleå University of Technology, Sweden

- Bialas, Andrzej, Research Network Łukasiewicz Institute of Innovative Technologies EMAG, Poland

\section{Program CommitTeE}

- Banach, Richard, University of Manchester, United Kingdom

- Bun, Rostyslav, Lviv Polytechnic National University, Ukraine

- Clarke, Nathan, Plymouth University, United Kingdom

- Cyra, Lukasz, DM/OICT/RMS (UN) 
- Daszczuk, Wiktor Bohdan, Warsaw University of Technology, Poland

- Felkner, Anna, Research and Academic Computer Network NASK

- Furnell, Steven, Plymouth University, United Kingdom

- Furtak, Janusz, Military University of Technology, Poland

- Gawkowski, Piotr, Institute of Computer Science, Warsaw University of Technology, Poland

- Grzenda, Maciej, Orange Labs Poland and Warsaw University of Technology, Poland

- Hämmerli, Bernhard M., Hochschule für Technik+Architektur (HTA), Switzerland

- Hasssaballah, M., South Valley University, Egypt

- Kapczynski, Adrian, Silesian University of Technology, Poland

- Krendelev, Sergey, Novosibirsk State University,JetBrains research, Russia
- MD Faisal, Mohammad, Integral University, India

- MD Rafiqul, Islam, School of Computing and Mathematics|Charles Sturt University

- Misztal, Michal, Military University of Technology, Poland

- Pańkowska, Małgorzata, University of Economics in Katowice, Poland

- Rot, Artur, Wroclaw University of Economics, Poland

- Stokłosa, Janusz, WSB University in Poznan, Poland

- Suski, Zbigniew, Military University of Technology, Poland

- Szmit, Maciej, University of Lodz, Poland

- Wahid, Khan Ferdous, Airbus, Germany

- Yahya, Eslam, Ohio State University, Columbus

- Zamojski, Wojciech, Wrocław University of Technology

- Zieliński, Zbigniew, Military University of Technology, Poland 


\title{
The general universal model of blockchain technology based on an analysis of some implementations
}

\author{
Polina Sazonova \\ Novosibirsk State University, JetBrains Research Laboratory \\ Sobolev Institute of Mathematics \\ Novosibirsk, Russia \\ Email: p.sazonova@nsu.ru
}

\begin{abstract}
First imlementation of blockchain technology was appeared in 2008, and 12 years later more than 2000 different implementations of it have appeared. After deep analysis we found that approaches for development blockchain technologies is fragmented, there are no common system of concepts and general model of technology. In this article we want to propose the general universal model and system of concepts for the blockchain technology irrespective of differenced of some implementations. Our approach is based on a technical analysis of the popular blockchains. The results of this work can be used by architects of new blockchains implementstions, by researchers to achieve theirs goals and also in educational process.
\end{abstract}

\section{INTRODUCTION}

\section{A. Blockchain definition}

B LOCKCHAIN technology has become popular due to the its properties such as openness, immutability, inability to delete stored data, decentralization and the ability to make decisions in an untrusted environment between equal participants in this network without the participation of a trusted party (trusted centre). Thus, blockchain uses in a wide variety of subject areas, especially in logistics, banking and public administration.

Blockchain is a type of decentralized system that collects, stores and manages data, in which:

- consensus will be reached in an untrusted environment;

- transactions are stored in a data structure called blocks, and each subsequent block stores the value of the hash function from the contents of the previous one;

- copies of the blockchain are stored at the same time by all its users and are automatically updated.

In this work, under the blockchain is meant a system that uses a chain of blocks as a technology for storing data. It provides ensures the immutability and integrity of the data stored in the blocks. Unlike centralized systems, where consensus can be achieved through a central node, blockchain technology allows to reach consensus in decentralized environment. Moreover, in the blockchain system, consensus can be

This work was supported by Math Centre in Academgorodok by agreement of The Ministry of Science and Higher Education of the Russian Federation number 075-15-2019-1613 and by JetBrains Research Cryptography Laboratory. reached when the network nodes are not authorized. It means that the probability of malicious nodes or Byzantine nodes [1] appearing on the network is increase. In decentralized networks with unauthorized (untrusted) nodes, a Sybil [2] attack may occur. It can happens when the node performing the calculations connects only to nodes controlled by the attacker, which entails incorrect behavior and consensus in making a decision that is beneficial to the attacker. Blockchain technology allows to make the right decisions in a decentralized network with untrusted nodes, provided that $51 \%$ of the nodes are not intruders.

\section{B. Introduction to history}

The first practical implementation of blockchain technology was done in 2008, it was described in the article by S. Nakomoto about digital monetary system Bitcoin [3]. Bitcoin is a protocol for exchanging digital money in a decentralized untrusted environment that allows to make transactions without the participation of third parties (trusted centre).

But before the publication of this article, it was made lots of reseaches influented over on the blockchain technology appearing. In 1982 D. Chaum proposed the blind signature algorithm and introduced the concept of digital money [4]. S. Haber and S. Shtornetta presented a theoretical description of the system for certifying immutability of documents, built on timestamps in 1991 [5]. The Proof of Work (PoW) mechanism was proposed by A. Back in the Hashcash project to prevent [6] spamming. The idea of smart contracts was proposed by N. Szabo in 1996 [7]. N. Szabo also proposed a protocol for digital money Bit-gold in 1998, which was published in 2005 [8]; it was based on bit-chain computation and used the PoW consensus mechanism. But the system was not implemented in practice and was vulnerable to the Sybil attack.

However, the first implementation of blockchain technology was created only as a part of the Bitcoin cryptocurrency project. Subsequently, new cryptocurrency systems began to appear, similar to Bitcoin. It was added data hiding mechanisms, such as in Zcash [9], transaction acceleration mechanisms, such as in Litecoin [10]. Currencies were created for various purposes, for example, providing a set of alternative 
DNS servers as in Namecoin [11]. The first implemented blockchain which was a platform for creating a smart contracts was Ethereum, created by V. Buterin in 2013 [12].

\section{Motivation of CReation a Blockchain TeChNOLOGy Model}

\section{A. Statement of the Problem}

An analysis of several hundred articles in Scopus on the topic of blockchain technologies showed that there are practically no scientific works that describes blockchain technology in general focused on its technical construction, covering all components of technology, regardless of specific implementations. In this direction it is worth highlighting this work [13], an overview of the blockchain technology components from the developers of the "Roadmap for the development of Distributed Ledger Technology (DLT)" in Russian Federation [14], an activity of the Geneva Telecommunication Standardization Sector Assembly (ITU) [15] and an activity of ISO/TC 307 committees [16]. But the results of most researchers work are not yet publicly available or have obvious flaws. This confirms the assumption that knowledge about technology is fragmented and the overall picture is not visible to researchers. This slows down the development of new technology implementations and makes it difficult to analyze new blockchains when we need to find real innovations, in contrast to the result of applying marketing tools.

\section{B. Methods, Purpose and Criteria of the Developed Model}

In this article the task of constructing a general universal model was to propose a model that would meet the following criteria: it would make it possible to make a universal description of current blockchain systems, answer questions about the structure of the system, and pose new questions to researchers and industry engineers. To build the model, an experimentalanalytical approach was used: based on existing software implementations of the blockchain technology, the components of the technology were analyzed, then the obtained components were generalized, and a system of concepts was formulated for them. Then it was shown that each specific technology implementation corresponded to the proposed model.

To make a general universal model, five popular blockchains were analyzed, which are independent implementations of platforms for developing decentralized applications and cryptocurrencies. Among them: Bitcoin [17], Ethereum [18], NEO [19], DASH [20], EOS [21]. The choice of these technologies is due to their relevance as platforms for the development of decentralized applications, the high level of readiness of the technology for application, its developed by community to support them and the availability of satisfactory documentation. The characteristics of the selected blockchains are presented in the table (cf. table I).

To solve this problem the general model of blockchain technology was developed. This model does not depend on specific implementations. A key components of blockchain technology were defined and their definitions were supposed with the aim of eliminating disagreements of interpretations.

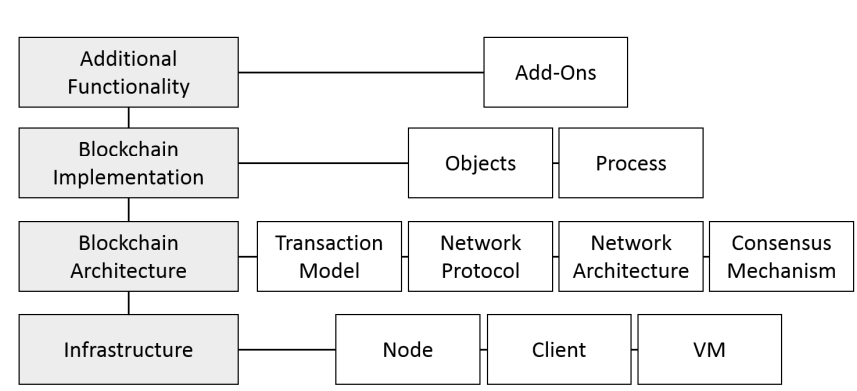

Fig. 1. Proposed blockchain technology model

The developed general model of blockchain technology is presented in the next section.

\section{Proposed Blockchain Technology Model}

For the five selected blockchains some documents as $\mathrm{s}$ technical documentations, technical concepts, «yellow papers» were analyzed. Common components that uniquely determine the blockchain technology were identified. These components are shown in the figure 1 and described in the text below.

At the first, basic, level of the model are the infrastructure components that ensure the functioning of the system. This is node - a single computer that performs actions on the network; client - software that implements the protocol of interaction with the blockchain; and virtual machine (VM) - a software system that emulates distributed work of a decentralized blockchain platform and executing decentralized applications and smart contracts.

At the second level, components are placed that ensure the functioning of the blockchain network. Depending on how this level is built, implementation features are established.

Network architecture - a combination of network nodes and a set of rules which uses for the the transmission of messages over the network. Blockchain networks can be single-layer or two-layer, public or private; they can have separation of nodes by roles.

Consensus Mechanism is a protocol that allows to reach an agreement between equal participants in a decentralized network. There are many implementations, but the most popular consensus is PoW, PoS, BFT and etc.

Transaction Model is a set of algorithms and features of design of the blockchain implementations that determine the method of conducting transactions and fixing the state of a distributed system. Currently, there are only two models uses in blockchains - UTXO or account model.

Network Protocol - the rules which uses for transmitted data over the network.

At the third level, objects and processes are located. This level arrangement depends on the implementation of the previous level. To begin with, we list objects, the presence of which is uniquely determined the blockchain technology. 
TABLE I

CHARACTERISTICS OF THE INVESTIGATED BLOCKCHAins

\begin{tabular}{|l|c|c|c|c|}
\hline Blockchain & Transaction validation speed & Block size & One block creation speed & Bandwidth \\
\hline Bitcoin & $78 \mathrm{Min}$. & $1 \mathrm{Mb}$ & $10 \mathrm{Min}$. & $3 \mathrm{TPS}$ \\
\hline Ethereum & $6 \mathrm{Min}$. & $1 \mathrm{Mb}$ & $15 \mathrm{Sec}$. & 20 (PoW), $400(\mathrm{PoA}) \mathrm{TPS}$ \\
\hline EOS & $1,5 \mathrm{Sec}$. & About $1 \mathrm{Mb}$ & $1 \mathrm{Sec}$. & $50000 \mathrm{TPS}$ \\
\hline NEO & $15 \mathrm{Sec}$. & About $1 \mathrm{Mb}$ & $15 \mathrm{Sec}$. & $1000-10000 \mathrm{TPS}$ \\
\hline DASH & $15 \mathrm{Min}$. & $2 \mathrm{Mb}$ & $1 \mathrm{Sec}$. & $28-56 \mathrm{TPS}$ \\
\hline
\end{tabular}

Block is a data structure uses to store data on the blockchain. The block stores transactions, network status, smart contracts, permissions to access data and other information.

Block chain is a data structure constructed by sequentially combining blocks into a chain. By storing the value of the hash function from the previous block, all blocks are strictly sequential, numbered by continuous numbering, the child block always refers to only one parent block.

Transaction is the minimum logically meaningful operation of the transfer or exchange of assets that makes sense and can only be completed in full. A transaction can transfer messages, actions, create a contract, and more.

Address (account, account) is a structure for identifying an active object on the network. Addresses uniquely determine the sender and recipient of the assets transferred to the blockchain network, all actions of the user in the network are associated with the address. Depending on the blockchain, the address can be either a string or a data structure, it can be associated with a user or with a smart contact.

Smart contract is a set of formalized rules implemented in the form of program code, the execution of which entails some events in the real world or digital systems. Smart contracts are not a mandatory component of the blockchain network, however, as practice has shown, contracts have become the main functional element of blockchain technology. Depending on the structure of the blockchain, smart contracts can be implemented either in Turing-complete languages or nonTuring-complete ones.

The objects listed above are part of the processes. The main processes taking place in the blockchain network are presented below.

Transactional life cycle: transaction signing process; broadcasting over the network; transaction verification; transaction completion. Including a transaction in a block: process of taking a set of transactions for a block; transaction validation; block signing process; sending a block to the network; block fixing in a common chain. Network Maintenance: consensus mechanism; network complexity regulation; selecting a chain that continues the block of several branches; payment for computing resources.

The fourth level defines additional functionality for blockchain networks that do not affect the internal architecture of the technology, but significantly expand its functionality. For example, mechanisms that provide increased speed and confidentiality of transactions, mechanisms for off-chain trans- actions, modules that protect blockchain against attacks by quantum computers, and others.

\section{CONCLUSIONS}

After analyzing the blockchain implementations and building model as a result, we can offer a method for considering each new technology being developed. To analyze the new blockchain implementation, first of all, we should pay attention to the transaction model. Currently, only two models are presented - UTXO and the accounts model. The transaction model affects on: the structure of blockchain blocks, the structure of addresses (accounts), the existence of smart contracts in this blockchain and the principles of their construction, approaches to fixing the state of the system. Next, we should pay attention to the number of layers in the blockchain network, identify the purpose of each of the layers, consider the consensus mechanisms used in each layer. This information will give us an understanding of the transaction validation process we can assume the bounds of transaction confirmation rate and network bandwidth. Based on this, we can suppose the requirenments to the necessary infrastructure to provide the network. The transactions rate is determined by the consensus mechanism, by the number of nodes involved in the transaction validation process and by the principles of working with orphaned blocks. The more stronger requirenments to network decentralization, the lower the transactions speed. The ability to create smart contracts is determined by the transaction model.

Using the results of this research we can explain approaches to the implementation of specific blockchain technologies. After researches we suppose that the majority of blockchain implementations are based on Bitcoin and Ethereum construction, and subsequently they were supplemented by some improvements at different levels. According to data obtained from open sources, it seems that the NEO blockchain consist of configuration of networks based on UTXO models and account models. We suppose that it makes in order to smooth out the limitations of the Bitcoin network, taken as the basis for NEO blockchain. This assumption was also made because the duplicate assets CNEO and CGAS seems artificial in these network. There is an assumption that the EOS and NEO blockchains are not blockchains, since the blockchain operates in an untrusted environment by definition, but for these networks the main transaction validators are authorized nodes, which suggests the centralization of these networks. 
The Dash blockchain ensures data confidentiality and transaction speed through mechanisms operating at the fourth level of the blockchain model.

\section{Results}

As a result of this work, the general model of blockchain technology was proposed. This model allows to make a universal description of current blockchains, answer some questions about components and links between it in the system, and pose new questions to researchers. In this work, it was proved that the proposed model does not depend on specific implementations of the five selected blockchains and suggest methods for considering each new blockchain implementation and explain approaches to the implementation. In the future, it is planned to investigate a larger number of different blockchains in order to confirm the correctness of the model and its quality, also we plan to show connections of blockchain technology to the environment.

\section{REFERENCES}

[1] L. Lamport, M. Pease, R. Shosta. "The Byzantine Generals Problem." ACM Transactions on Programming Languages and Systems 4, p. 3, pp. 382-401, 1982

[2] J. R. Douceur. "The sybil attack." International workshop on peer-topeer systems. Springer, Berlin, Heidelberg, 2002, pp. 251-260.

[3] S. Nakamoto. "Bitcoin: A Peer-to-Peer Electronic Cash System." The Cryptography Mailing List, 2008, https://bitcoin.org/bitcoin.pdf.

[4] D. Chaum. "Blind Signatures for Untraceable Payments." Advances in Cryptology Proceedings of Crypto 82, Plenum, 1982, pp. 199-203, 1982

[5] S.Haber, W.S. Stornetta. "How to time-stamp a digital document." $J$. Cryptology 3. 1991, pp. 99-111.
[6] A. Back. Mail "Hash cash postage implementation". The Cypherpunks Mailing List. https://cypherpunks.venona.com/date/1997/03/msg00774. html.

[7] N. Szabo. "Smart Contracts: Building Blocks for Digital Markets." A partial rewrite of the article which appeared in Extropy No 16, 1996, http://www.alamut.com/subj/economics/nick_szabo/ smartContracts.html.

[8] N. Szabo. "Bit gold." Unenumerated: N. Szabo's blog, 2005, https://web.archive.org/web/20060329122942/http://unenumerated. blogspot.com/2005/12/bit-gold.html.

[9] Zcash - a privacy-protecting, digital currency, https://z.cash/.

[10] Litecoin - decentralised money, https://litecoin.com/en/.

[11] Namecoin - a trust anchor for the Internet, https://www.namecoin.org/.

[12] Ethereum - a global, open-source platform for decentralized applications, https://ethereum.org/ru/

[13] H.Y. Paik, X. Xu, H. M. N. Dilum Bandara, S. U. Lee, S. K. Lo. "Analysis of Data Management in Blockchain-Based Systems: From Arcitecture to Governance." IEEE Access, 2019, t.7, pp. 186091-186107, https://ieeexplore.ieee.org/stamp/stamp.jsp?tp=\&arnumber=8938787.

[14] Ministry of Digital Development, Communications and Mass Media of the Russian Federation. "Roadmap for the development of Distributed Ledger Technology (DLT).” https://digital.gov.ru/ru/documents/6670/.

[15] ITU's Telecommunication Standardization Sector (ITU-T). "ITU-T Focus Group on Application of Distributed Ledger Technology." https: //www.itu.int/en/ITU-T/focusgroups/dlt/Pages/default.aspx\#.

[16] Technical cometees ISO/TC 307. "Blockchain and distributed ledger technologies." https://www.iso.org/committee/6266604.html.

[17] Bitcoin developers documentation, https://developer.bitcoin.org/.

[18] G. Wood. Ethereum: a secure decentralized generalized transaction ledger, https://ethereum.github.io/yellowpaper/paper.pdf.

[19] Technical Specification for NEO Blockchain, https://github.com/ neoresearch/yellowpaper.

[20] E. Duffield, D. Diaz. Dash: A Payments-Focused Cryptocurrency. https: //github.com/dashpay/dash/wiki/Whitepaper.

[21] EOS Developer Portal, https://developers.eos.io/. 


\section{$2^{\text {nd }}$ Special Session on Data Science in Health, Ecology and Commerce}

D ATA Science in Health, Ecology and Commerce is a forum on all forms of data analysis, data economics, information systems and data based research, focusing on the interaction of those four fields. Here, data-driven solutions can be generated by understanding complex real-world (health) related problems, critical thinking and analytics to derive knowledge from (big) data. The past years have shown a forthcoming interest on innovative data technology and analytics solutions that link and utilize large amounts of data across individual digital ecosystems. First applications scenarios in the field of health, smart cities or agriculture merge data from various IoT devices, social media or application systems and demonstrate the great potential for gaining new insights, supporting decisions or providing smarter services. Together with inexpensive sensors and computing power we are ahead of a world that bases its decisions on data. However, we are only at the beginning of this journey and we need to further explore the required methods and technologies as well as the potential application fields and the impact on society and economy. This endeavor needs the knowledge of researchers from different fields applying diverse perspectives and using different methodological directions to find a way to grasp and fully understand the power and opportunities of data science.

This is a joint track by WIG2, the Scientific Institute for health economics and health service research, the Information Systems Institute of Leipzig University and the Helmholtz Environmental Research Institute.

\section{TOPICS}

We embrace a rich array of issues on data science and offer a platform for research from diverse methodological directions, including quantitative empirical research as well as qualitative contributions. We welcome research from a medical, technological, economic, political and societal perspective. The topics of interest therefore include but are not limited to:

- Data analysis in health, ecology and commerce

- (Health) Data management

- Health economics

- Data economics

- Data integration
- Semantic data analysis

- AI based data analysis

- Data based health service research

- Smart Service Engineering

- Integrating data in integrated care

- AI in integrated care

- Spatial health economics

- Risk adjustment and Predictive modelling

- Privacy in data science

\section{TECHNICAL SESSION CHAIRS}

- Franczyk, Bogdan, University of Leipzig, Germany

- Militzer-Horstmann, Carsta, WIG2 Institute for health economics and health service research, Leipzig, Germany

- H\&auml;ckl, Dennis, WIG2 Institute for health economics and health service research, Leipzig, Germany

- Bumberger, Jan, Helmholtz-Centre for Environmental Research - UFZ, Germany

- Reinhold, Olaf, University of Leipzig / Social CRM Research Center, Germany

\section{Program CommitTeE}

- Alpkoçak, Adil, Dokuz Eylul University

- Cirqueira, Douglas, Dublin City University

- da Rocha Cirqueira, Douglas, Dublin City University

- Dey, Nilanjan, Techno India College of Technology, India

- Kossack, Nils, Head Mathematics and Statistics, WIG2 Institute for Health Economics and Health Service Research

- Kozak, Karol, Fraunhofer and Uniklinikum Dresden, Germany

- Popowski, Piotr, Medical University of Gdańsk, Poland

- Sachdeva, Shelly, National Institute of Technology Delhi, India

- Wasielewska-Michniewska, Katarzyna, Systems Research Institute of the Polish Academy of Sciences, Poland

- Wende, Danny, WIG2 Institute for Health Economics and Health Service Research And Technical University Dresden 



\section{Interactive Online Reporting of Registry of Tuberculosis}

\author{
Matěj Karolyi, Tereza Nováková, Petr Panoška, \\ Martin Komenda, Jiř́ Jarkovský \\ Institute of Health Information and Statistics of the \\ Czech Republic and Institute of Biostatistics and \\ Analyses, Faculty of Medicine, Masaryk University - \\ joint workplace, \\ Palackého nám. 4, 128 01, Praha 2, Czech Republic \\ Email: \{karolyi, novakova, panoska, komenda, \\ jarkovsky\}@iba.muni.cz
}

\author{
Jiří Wallenfels \\ National Tuberculosis Surveillance Unit, Hospital \\ Bulovka, \\ Budínova 2, 18000 Praha 8-Bulovka \\ Email: jiri.wallenfels@bulovka.cz
}

\begin{abstract}
The existence of health registers at the national level allows for a systematic and continuous control of the state of healthcare, the digitalisation of certain services and the overall improvement of the state of care. The data collected in such registers are then analysed and visualised. Thanks to this, it is possible to effectively evaluate various indicators and thus pass on a comprehensive picture to all stakeholders involved in the health system - legislators, healthcare providers and patients. The Registry of Tuberculosis (RTBC) is one source of data. The article describes this data source as well as the methods that lead to the creation of a freely available online interactive tool. Published visualisations offer a view of the data from several angles and deal with various subtopics of tuberculosis. Finally, particular results and possible improvements are presented.
\end{abstract}

\section{INTRODUCTION}

$\mathrm{T}$ HERE has been a recent trend to create complex visualization tools and data analyses from public healthcare datasets, which are stored inside various national registries. Especially for monitoring of crucial and serious diseases, the development of analytical and reporting tools over national health registries leads to an increase of knowledge and the research for problems of experts who are looking for current trends and who predict the possible trends. In the Czech Republic, such datasets are analysed and published by leading government institutions: Ministry of Health (MH), National Institute of Public Health (NIPH), Institute of Health Information and Statistics (IHIS), public health universities and others. Following information systems and have already been developed and published and can be considered as related work:

- Czech Childhood Cancer Information System ${ }^{1-}$ based on available data sources, Czech Childhood Cancer Information System improves the healthcare professionals' awareness of childhood cancers and promotes their education in this area. The target groups involve paediatricians, non-specialized paediatric departments of healthcare facilities and professionals in both basic and applied research, as well as civic associations of patients and their family members [1].

1 https://ccc-is.uzis.cz/index-en.php
- Comprehensive Cancer Care Network ${ }^{2}$ - a pilot model-established in two Czech regions: Vysočina Region and South Moravian Region. The pilot model covers all components of cancer care: from cancer prevention and organized screening programmes through standard diagnostic and treatment procedures to follow-up plans; specialized rare tumours-focused care as well as palliative are also included [2].

- Map of Medical Equipment ${ }^{3}$ - the aim is to provide a clear visualization of information on the availability of individual categories of medical equipment at all health service providers that, according to the law, submitted the Annual Report on the instrumentation of a medical facility. The data used are based on the Program of Statistical Surveys of the Ministry of Health of the Czech Republic [3].

- The National Database of Palliative Care ${ }^{4}$ mapping and description of the end-of-life care in the Czech Republic. The portal aims to provide a complex view on the end-of-life issues and to contribute to the work of healthcare workers who make efforts to alleviate suffering and to improve the quality of life of tens of thousands of patients dying each year.

- National Registration of Health Services Providers ${ }^{5}$ - the web portal provides a complete overview of all healthcare providers in the Czech Republic. It provides data on the profile and scope of care for medical facilities, contains information on the availability of medical facilities and other detailed information [4].

Tuberculosis disease (TB) and other mycobacterial infections have been monitored for a long time in the Czech Republic [5]. A newly developed visualization portal of the Registry of Tuberculosis (RTB) is part of this effort and belongs to the group of previously presented web portals. The portal is intended for experts in the field, but also for the

\footnotetext{
2 https://ccen.onconet.cz/

$3 \mathrm{https}: / /$ zt.uzis.cz/

$4 \mathrm{https}: / /$ www.paliativnidata.cz/index-en.php

5 https://nrpzs.uzis.cz/
} 
general public, who can observe particularly the time trends of TB and other mycobacterial infections.

The web portals and online visualizations described above are based on data from the Czech National Health Information System (NHIS). NHIS comprises dozens of health registries and information systems and its existence is given by Czech legislation. Processing and publishing of health data for the purpose of obtaining clear information about the extent and quality of provided health services belong to the main services provided by NHIS. From the perspective of online data presentation describing selected domain of NHIS, the proper interpretation of given information must be in compliance with two aspects: (i) data from health registries must be processed, aggregated and visualized anonymously (with no direct identification of any patient record), (ii) potential combination of descriptive attributes in a form of dynamic filters over available datasets like sex, age, and location must never lead to show too low total number of cases per geographic unit (district or town).

Nowadays, interactivity is one of the most required features of web-based applications guaranteed and produced by $\mathrm{MH}$ and IHIS (e.g., connected with epidemiological data [6]), which present various results and data overviews [1]-[3]. Modern layout, attractive user interface, overview customization, and data filtering bring an unique way to properly deliver given information to the final user/target group. With the respect to this approach, an interactive online reporting of the tuberculosis registry has been designed, developed and deployed.

\section{A. Czech Registry of Tuberculosis}

The Registry of Tuberculosis (RTB) is a part of Czech public health registries maintained by MH, IHIS, NPIH and Regional Public Health Authorities (RPHA) ${ }^{6}$. In the Czech Republic, reporting of all detected cases of tuberculosis to the RTB is obligatory by law. Therefore, the registry includes all individuals with active tuberculosis or other mycobacteriosis and all persons treated in groups of active and inactive tuberculosis or other mycobacteriosis which were diagnosed in the Czech Republic. As the Information System of Bacillary Tuberculosis (ISBT) has recently become an integral part of this registry, RTB is a very complex data source for this type of infection. Data from RTB are processed by IHIS and a statistical summary publication reflecting the current epidemiological situation in the Czech Republic is published once per year, entitled Basic Overview of Tuberculosis Epidemiology in the Czech Republic. Together with the National Tuberculosis Surveillance Unit, IHIS also maintains contacts with international organizations.

\section{II.METHODS}

The whole process of design and development is divided into different steps, which are iteratively repeated if needed. During the whole period we keep in mind the verified methodology for data mining called Cross Industry Standard Process for Data Mining (CRISP-DM) [7] in a domain- specific environment [8]. This helps us to standardize our endeavour and optimize the delivery of the final product. Our development process often leads to the detection of low data quality. Therefore, we involve techniques for the improvement of the underlying dataset. This ensures continuous fixing of data entries even in the source Registry of Tuberculosis.

\section{A. Data description}

Data entering the web application are transmitted from data analysts to software developers in csv format. There are also several supporting files created, including the list of variables and its character (binary, numeric, string, etc.) and possible values, a logical framework displaying the allowed and forbidden usage of filters in given analyses, and a summary of required graphic outputs for each analysis.

The final dataset itself includes numerous descriptive variables, such as demographic data (age, gender, region of residence, etc.), important report dates (for example year of reporting or year of death) and a set of selected clinical parameters (type of infection, diagnosis by ICD-10 code, etc.). Apart from the main dataset, two separate datasets exist as a data source for the analyses of treatment results and susceptibility to antituberculotic drugs. Only filtering by year of reporting is available for these analyses.

The combination of those datasets is quite unique worldwide (according to our knowledge only few countries present their TB data in online and publicly accessible form). Therefore, our newly developed online reporting tool could have ambition to become an inspiration to similar teams in other countries which want to present their own TB data.

\section{B. Methods of analysis}

To display real numbers of cases of $\mathrm{TB}$ and other mycobacterioses, it is necessary to apply standardised prefiltration processes. Based on specified diagnosis (by ICD-10 coding system), year of reporting and several other variables were identified as key ones to describe relevant (correct and complete) records in RTB data. Two standard statistical methods were subsequently applied, based on the character of individual analysis.

Firstly, the frequency analysis is used to determine the absolute numbers of cases, which are displayed for instance in analyses "Time trends - cases" or "Time trends-deaths from TB". In analyses where percentages were preferable, the relative frequency analysis is employed, such as "Age distribution", "Country of birth" or "Mycobacterial examination", where the percentages provide a better perspective.

\section{Technological background and deployment}

From a technical point of view, the portal consists of a relational database, backend (server-side) framework and frontend (client-side) framework. A complete architecture is shown in Fig. 1.

\footnotetext{
${ }^{6}$ https://www.uzis.cz/res/f/008271/tbc2018-en-a2b.pdf
} 


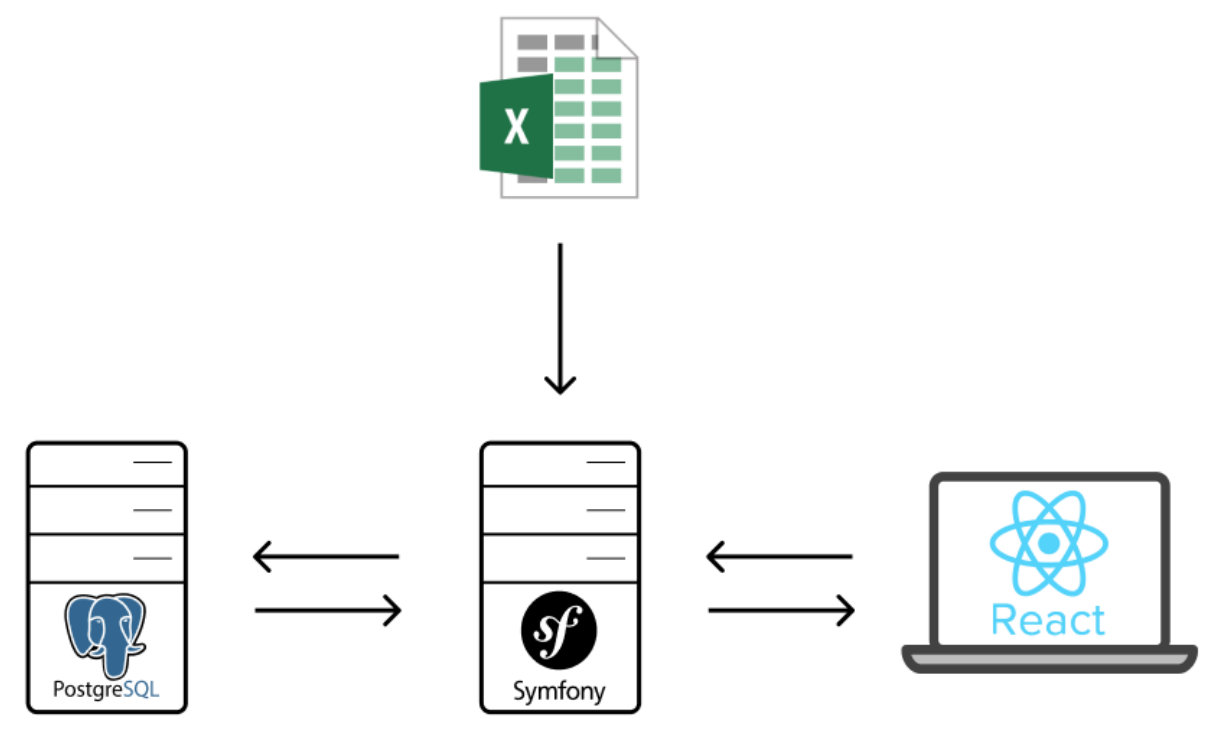

Fig. 1 Portal architecture schema

PostgreSQL is used due to our previous experience and simultaneous usage in other web services. Selection of the backend platform was also based on existing knowledge and current technical environment setup; thus we use the Symfony framework. The choice of frontend framework is driven by the need of dynamic content rendering and usage of several javascript libraries for data visualization. For this reason, React was selected. Fig. 1 also demonstrates that the data discussed earlier are served to Symfony, where their import to the database is performed. Data is mapped to PHP entities with the Doctrine Object Relational Mapper (ORM) framework, which is a suitable solution for transmitting data between database and Symfony.

When the data is ready to be queried by the backend server, API endpoints are implemented for each analysis, in which data preprocessing is done. Filters selected by the user are applied and data aggregation and normalization are performed during this process. Final data are served in JSON format to the client.

Key aspect of choosing React and its major advantage is re-rendering content according to user analysis type and filters' selection (application state) without reloading the whole page. Typically, when the user loads an analysis page (view), visualization and also static data are served to the client and the page is rendered. For the next user interactions with the view, only the visualization data are responded by the server and visualization components are updated. Most of the components are charts from the NVD3 javascript library, which has a high-level API for convenient components updates. As a result, users can work with the analysis without unnecessary reloading, and chart components smoothly update their values, which is an important user experience improvement.

\section{RESULTS}

The Interactive Registry of Tuberculosis Data Viewer is a web-based tool that allows health professionals to gain an overview of the state of the disease from various perspectives. This tool is freely accessible at https://tbc.uzis.cz/ via a web browser. All currently available analyzes are available without the need to log in and at the time of publication of the article contain data from 2000 to 2019 .

Current structure of the portal allows further expansion with potential further analyzes and graphical outputs. The currently available ones are further supplemented by static outputs directly on the IHIS.

\section{A. Portal structure}

The portal is divided into several sections, where users can find the content connected with the topic of tuberculosis disease and other mycobacterial infections in the Czech Republic.

\section{Information about the registry}

Basic static pages contain introductory information and an overview of the context in which the portal has been developed. Part of this overview is also a basic introduction to the Registry of Tuberculosis, whose establishment and operation are regulated by a governmental decree.

One of the important outputs are yearbooks of tuberculosis. The publication "Basic Overview of Tuberculosis Epidemiology in the Czech Republic" has been published regularly by IHIS CR since 2016 (with data for 2015) and indirectly follows the publication "Tuberculosis and Respiratory Diseases" which contains data from 1960 to 2014. It is a selection of the most important overview tables characterizing the occurrence of reported cases of 
tuberculosis and other mycobacterioses in the Czech Republic. These documents are available on the IHIS CR website .

\section{Data viewer}

Each analysis has its own separate visual presentation. Two analyses are available only for the Czech Republic as a whole, but most of them offer a region-based view on data. Analysis outputs can also be narrowed through several filters, which comprise basic demographic variables as well as selected clinical parameters.

Current analyses (views) over the following topics:

- Time trends - cases

- Time trends - deaths from TB

- Age distribution

- Nation of birth

- ICD diagnoses

- Proportion of previously treated patients

- Tests for mycobacterial infection

- Treatment results

- Susceptibility to antituberculotics

\section{B. Selected data visualizations}

In this paper, only a subset of the portal content is shown and described; three the most dissimilar were selected for demonstration. The rest is available and freely accessible online. Visualization outputs are generated and displayed online; however, the user is also allowed to download figures and tables to their computer.
Time trends-cases

First analysis called "Time trends - cases", which shows the number of detected cases of TB and other mycobacterial infections, is displayed as a time series line graph. That allows the user to explore the development of these infections over the whole period 2000-2019. Even more illustrative is the map, showing abundance of the diseases in Czech regions.

The output of this analysis shows a time series of cases reported in the Registry of Tuberculosis. Using the tabs, it is possible to switch between graphical and tabular overviews for the Czech Republic and for individual regions, and the cartogram. The results can be further filtered using selected parameters from the "filtering" panel. Fig. 2 shows the line chart of the TB incidence trend from 2000 to 2018 in a group of patients over 60 years.

\section{Age distribution}

When looking at the output of the "Age distribution" analysis, represented by a histogram displaying the relative numbers in 5 -year age categories, one can easily determine the age groups in which TB occurs with the highest frequency. When we divide the whole country to particular regions, a set of 14 boxplots is displayed (one boxplot for each region).

The same as in the previous case, it is possible to switch between graphical and tabular overviews for the Czech Republic and for individual regions. The results can be further filtered on demand. Fig. 3 shows a histogram of all age groups of Czech TB patients in a reduced time period.

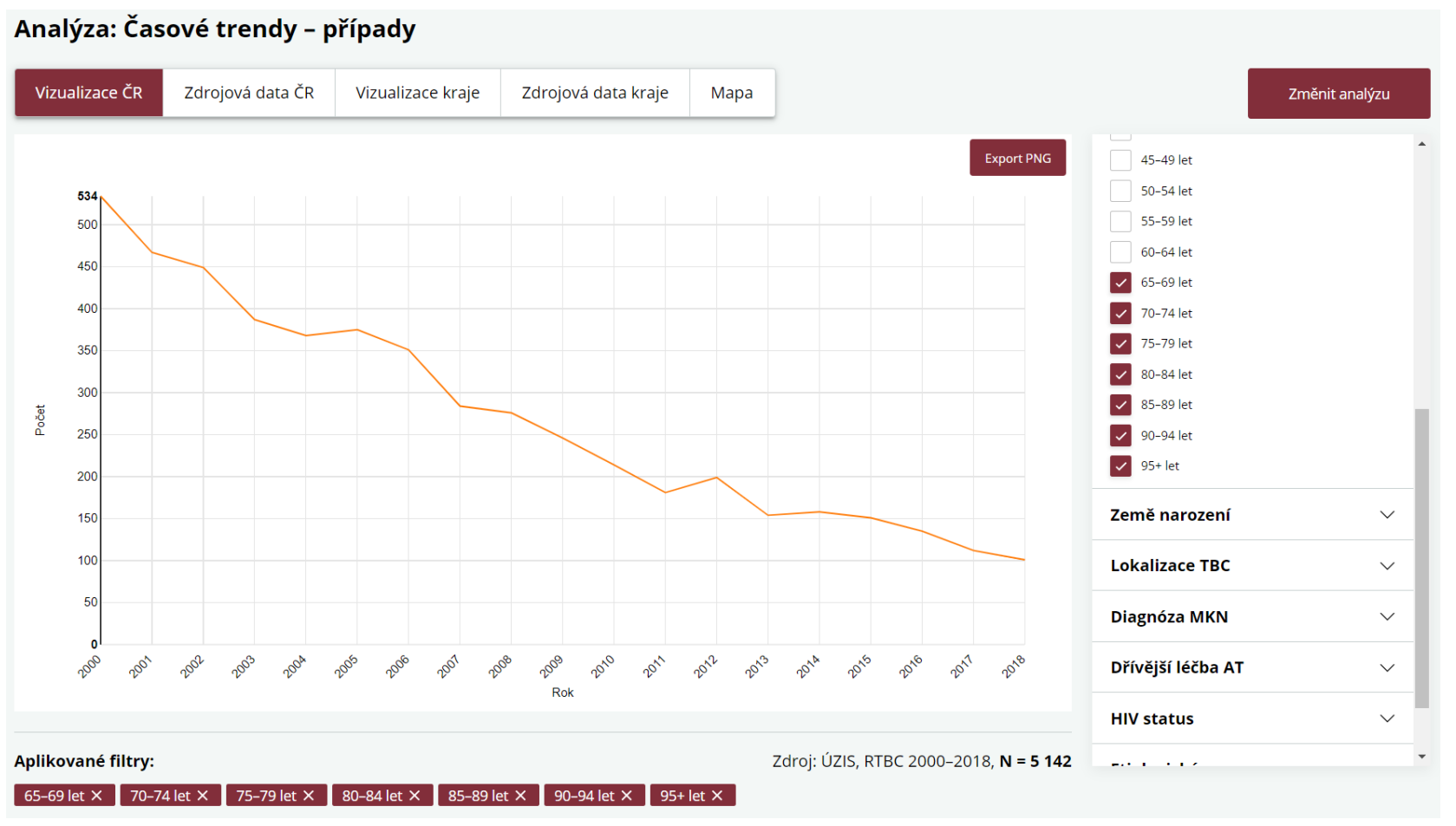

Fig. 2 Trend of TB disease from 2000 to 2018 in a group of patients older than 60 years (Czech only)

${ }^{7}$ https://www.uzis.cz/index.php?pg=registry-sber-dat--ochrana-

verejneho-zdravi--registr-tuberkulozy\#publikace 


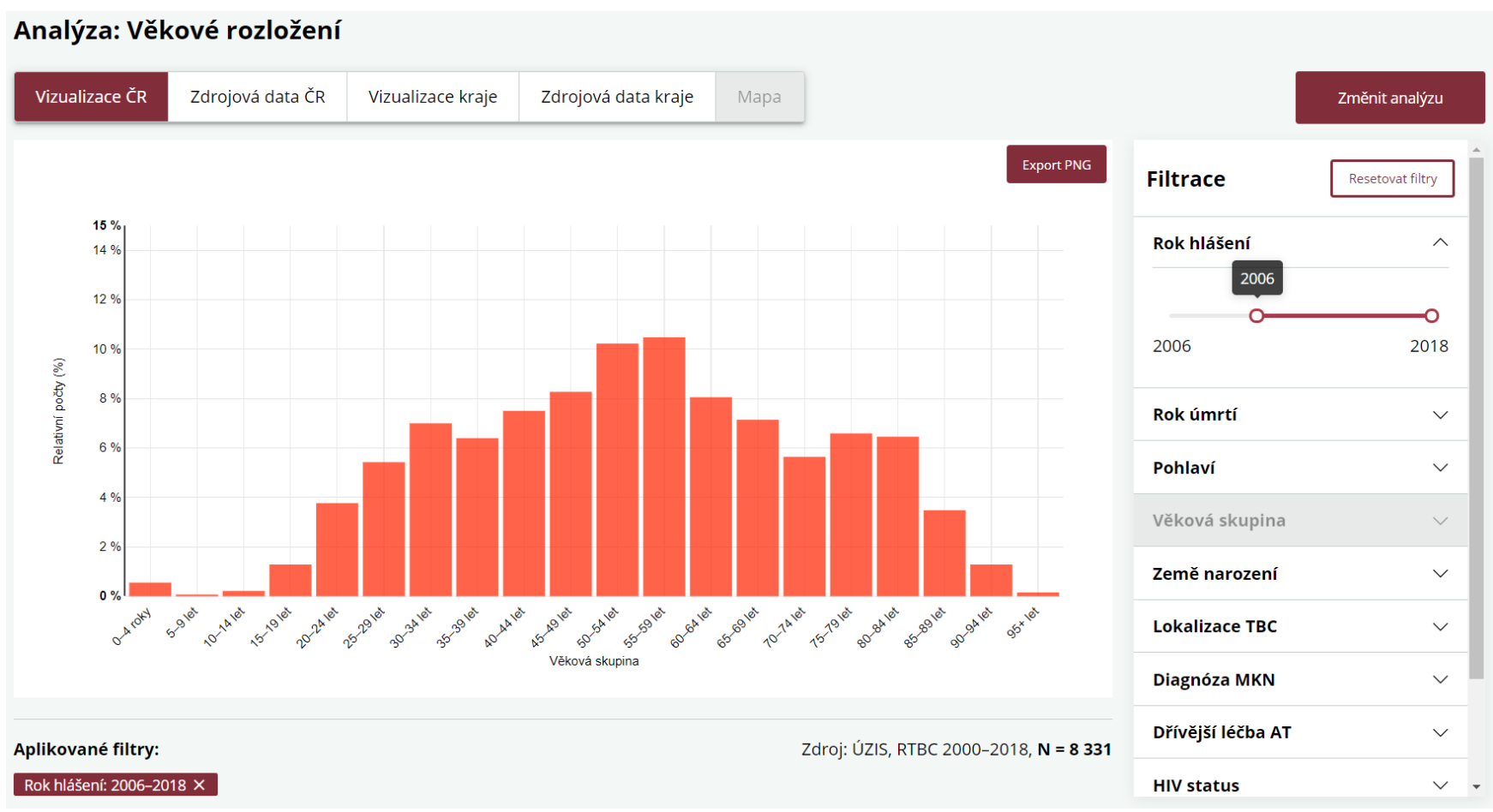

Fig. 3 Histogram of all age groups of czech TB patients (Czech only)

Tests for mycobacterial infection

The analysis "Tests for mycobacterial infection" is available for TB cases only and shows a barchart of relative frequencies of possible examination results-culturally positive TB, culturally positive TB verified from sputum or laryngeal swab (LS), and sputum smear microscopy-positive TB. The output of this analysis allows the user to compare the total number of cases of tuberculosis in the Registry of Tuberculosis with the number of cases of tuberculosis according to possible results of mycobacterial tests.

Fig. 4 shows the analysis of regions according to results of mycobacterial testing in 2018. The presented figure does not contain all regions.

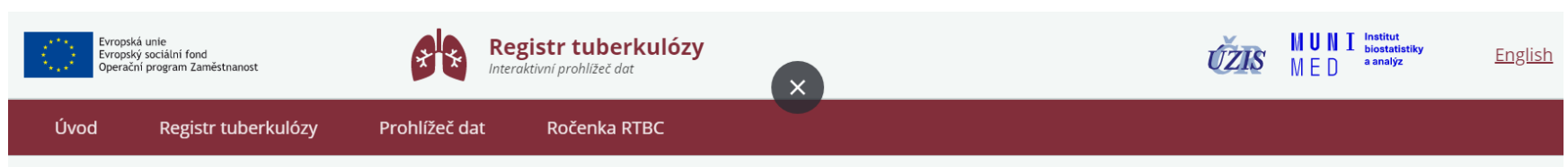

\section{Analýza: Mykobakteriologické vyšetření}

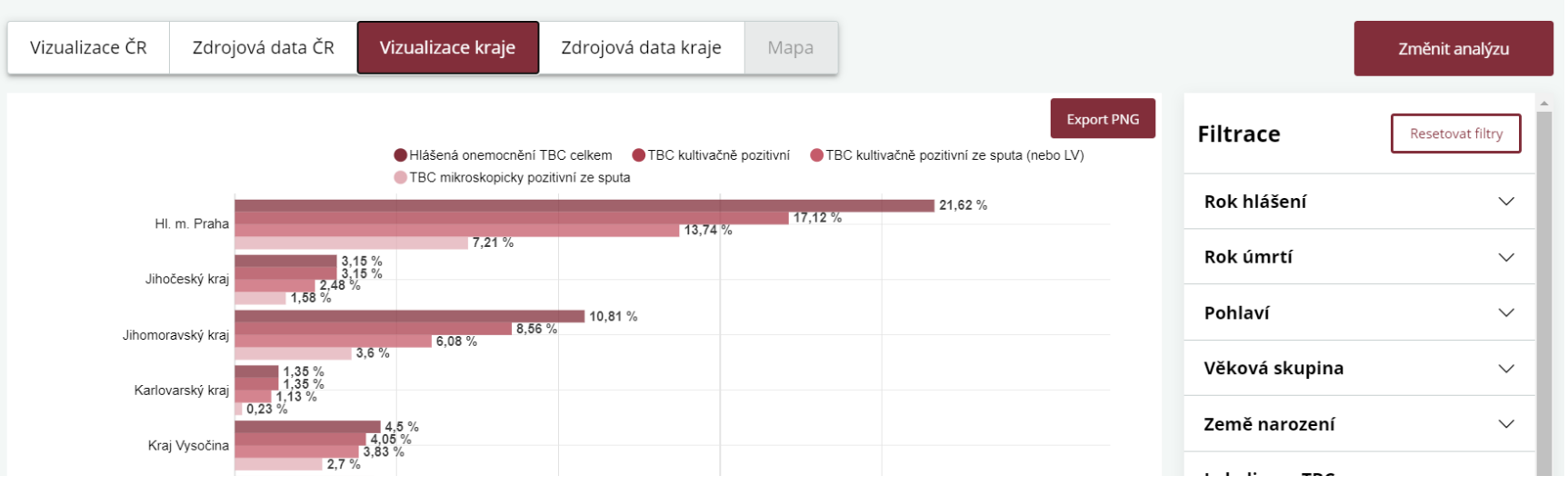

Fig. 4 Analysis of regions according to mycobacterial testing results (Czech only) 


\section{DISCUSSION}

In this article, we describe a web application tool for the exploration of data from the RTB. Users of this portal can choose between various analyses-from the basic ones based on demographic characteristics to some more advanced ones, which are suitable for experts, such as doctors or other experts. Worldwide, only few countries present their tuberculosis data and to our knowledge no country reports the cases of other mycobacterial infections in a form of web portal. This online tool is therefore unique in combining the two infections together in one application.

However, there are several limitations as well. Firstly, it is possible to access data from the year 2000 onwards only. When we compare this tool with similar applications based on TB data from the Netherlands ${ }^{8}$, one can see a longer time period to display. Secondly, the outputs of analyses are currently presented in absolute numbers and/or percentages only. We plan to add the possibility to present data as relative incidence. Thirdly, in comparison to the Dutch portal, data about latent TB infection (LTBI) are missingthis is due to the fact that the surveillance system of LTBI has still not been established in the Czech Republic. Also, the offer of possible outputs seem to be more extensive on the Dutch website, but since the Czech one has been intended to address the general public too, we decided to show the most important parameters only.

Another way to improve the comparability of data between regions or even other countries can be achieved if some the values are given as relative (e.g., per 100000 inhabitants).

Currently, the web portal is intended to be updated once per year by adding the definitive data for the previous calendar year. Alternatively, we consider more frequent updates with the use of preliminary data or even "rolling" updates reflecting the continuous reporting to RTB in real time.

We believe that in future this web portal could be considered as an example of data presentation for other infectious diseases that are mandatorily reported to the Information System of Infectious Diseases?

\section{CONCLUSION}

As a result of multidisciplinary cooperation, which included three important groups - experts in the field of tuberculosis and other mycobacterioses, data analysts, and software developers - we developed a freely available tool displaying visualizations of data from the National Tuberculosis Registry in the Czech Republic, which has a great potential to be beneficial for both professional and general public. The tool is based on modern technologies, which makes future long-term sustainability as well as scalability and extensibility much easier.

The content of the portal has already been reviewed several times by the professional community; their feedback was taken into account and the important points and comments were also implemented retrospectively. At the time of writing this paper, the content is only available in the local language version. We are planning a translation to English.

The data update is scheduled for an annual cycle. Within the datasets, there is no intention to change the data structure over the years; only new annual datasets will be added. However, if necessary, it will be possible to adapt to possible changes in the source registry (e.g., as a result of adjustments to data collection from healthcare providers).

\section{REFERENCES}

[1] D. Krejčí et al., 'Webový portál pro analýzu a vizualizaci dětských onkologických dat dostupných v ČR', 2019, Accessed: Jul. 01, 2020. [Online]. Available: https://www.med.muni.cz/veda-avyzkum/publikacni-cinnost/1576436.

[2] L. Dušek, J. Mužík, M. Karolyi, M. Šalko, D. Malúšková, and M. Komenda, 'A Pilot Interactive Data Viewer for Cancer Screening', in Environmental Software Systems. Computer Science for Environmental Protection, Cham, 2017, pp. 173-183, doi: 10.1007/978-3319-89935-0_15.

[3] M. Karolyi, J. Krejčí, J. Ščavnický, R. Vyškovský, and M. Komenda, Tools for development of interactive web-based maps: application in healthcare. Václav Skala - UNION Agency, 2019.

[4] 'Legislation - ÚZIS ČR'. https://www.uzis.cz/index-en.php? pg=nhis--legislation (accessed Jul. 01, 2020).

[5] Tuberkulóza a respiračni nemoci 1998. Ústav zdravotnických informací a statistiky ČR, 1999.

[6] M. Komenda et al., 'Complex Reporting of the COVID-19 Epidemic in the Czech Republic: Use of an Interactive Web-Based App in Practice', J. Med. Internet Res., vol. 22, no. 5, p. e19367, 2020, doi: $10.2196 / 19367$

[7] F. Martínez-Plumed et al., 'CRISP-DM Twenty Years Later: From Data Mining Processes to Data Science Trajectories', IEEE Trans. Knowl. Data Eng., pp. 1-1, 2019, doi: 10.1109/ TKDE.2019.2962680.

[8] O. Niaksu, 'CRISP data mining methodology extension for medical domain', Balt. J. Mod. Comput., vol. 3, no. 2, p. 92, 2015.

8 https://www.tbc-online.nl/eng/index.html

9https://www.uzis.cz/index.php?pg=registry-sber-dat--ochrana-verejneho-zdravi--informacni-system-infekcni-nemoci (czech only) 


\section{Conceptualization of an AI Based Assistant to Support the Compatibility of Family and Paid Work}

\author{
Philippe Krajsic \\ Institute for Applied Informatics (InfAI) \\ Goerdelerring 9, 04109 Leipzig, Germany \\ Email: krajsic@infai.org \\ Bogdan Franczyk \\ Uniwersytet Ekonomiczny we Wroclawiuul \\ Komandorska 118/120, 53-345 Wroclaw \\ Email: bogdan.franczyk@ue.wroc.pl
}

\author{
Martin Schieck, Ingolf Römer, Silvia Torres Landaverde \\ Information Systems Institute \\ Leipzig University \\ Grimmaische Straße 12, 04109 Leipzig, Germany \\ Email: \{schieck, roemer, torres\}@wifa.uni-leipzig.de
}

\author{
Carsta Militzer-Horstmann \\ WIG2 Institut \\ Scientific Institute for Health Economics \\ and Health System Research \\ Markt 8, 04109 Leipzig, Germany \\ Email: carsta.militzer-horstmann@wig2.de
}

\begin{abstract}
The goal of the presented intelligent AI-based assistant is to simplify the reconciliation of paid work and family. In times of the corona pandemic and the challenges it poses, solutions are needed to mitigate the impact on workers and families and to strengthen the compatibility of work and family life. We are developing a conceptual reference model of proven methods and technologies for the generation of information that can be queried by interested parties via an bot service, thus simplifying professional and family challenges. Essentially, we provide a framework and starting point for research, development, and evaluation of an AI-based chatbot for the reconciliation of paid work and family.
\end{abstract}

Index Terms-Artificial Intelligence, Chatbot, BERT.

\section{INTRODUCTION}

$\mathbf{I}$ $\mathrm{N}$ HARDLY any other sector the reconciliation of family and professional obligations is as difficult as in the care and medical services. The demands on personnel in the modern health care system are higher than ever before. In addition to alternating shift work and immense psychological and physical strain at work, most of them also bear responsibility in the private sector-whether it is child care or caring for relatives, possibly made even more difficult by their own illness, pregnancy or separation from their partner. Particularly in respect of the current pandemic situation (Corona Virus / COVID19) and the associated strain on personnel in the care and health sector, this topic is more relevant than ever before. As this is most likely to lead to a permanent change in the way the care and health sector works, and as the reconciliation of paid work and family life brings with it lasting challenges, particularly in this area, solutions must be designed to ease the burden on those involved in this sector. The work presents a technical approach that should be communicated to the scientific community as soon as possible in order to solve

This work was funded by the European Social Fund as well as co-financed by tax revenues on the basis of the budget adopted by the Saxon State Parliament within the project PFaDi — Platform for Family-Related Services the described challenges. Starting from this initial situation of a difficult compatibility of the different areas of life of the described group, this research project is looking for a databased answer, which provides individual, fast, and qualified support in every life situation. This investigation is based on the research project PFaDi (Platform for Family-Related Services). Thanks to bookable services for all situations in life, a unifying calendar function as well as information and links to official applications, guides, and contact addresses, this online platform makes it possible to coordinate the everyday life of families and to support the compatibility of family and professional obligations.

The progress made in the field of intelligent technologies has generated high social expectations. For example, intelligent text and speech recognition have gained high acceptance due to their low threshold (e.g., Alexa). Chatbot applications slick interactions between services and people, improving customer experience. Simultaneously, chatbots offer companies various opportunities to enhance the customer's loyalty and ensure operational efficiency by minimizing the surplus cost of customer service. Constant availability, quick answers and ease of use also contribute significantly to their attractiveness [1], [2].

For a dynamic information generation, the following research question emerges: How could an approach for an appropriate architecture for dynamic interactions in a chatbot context look like?

The remainder of the paper is structured as follows: Section II presents the initial situation in the area of reconciling family and paid work. Section III presents the related work in the area of artificial intelligence, text classification, and regulatory hurdles. In Section IV the reference model and its high-level components are described. In section $\mathrm{V}$ the main component of the reference model, BERT, is described in detail. Section VI explains the pre-training of the BERT model. The final section VII concludes the paper and presents future work. 


\section{INITIAL SITUATION}

Family and paid work can then be regarded as compatible if the conditions allow the acting person to delimit a stableflexible system of areas of action, in which there is scope for planning the transitions between areas of action; compatibility is thus essentially influenced by the ability to regulate the areas or the scope for action, time, and decision-making given there [3]. The issue of reconciling paid work and family/care is often dealt with in the context of the so-called work-life balance. This concept, which deals with the balance between paid work and the rest of life, explicitly includes the area of leisure time and is therefore somewhat broader in scope [4]. Reconciling paid work and family life can also be seen in the context of achieving greater gender equality in the company. While more men are involved in child care and upbringing today than in previous decades, a large part of family and care work is still performed by women. Women are thus exposed to a greater double burden than men in pursuing their professional careers. Family and care work not only contributes to the fact that women are less likely to work, it also makes it more difficult to realise career opportunities. The latter is expressed above all in the fact that women are underrepresented in management positions [5]. For this study, it must be noted that according to a WHO analysis of March 2019 on the gender distribution in the health sector in 104 countries, $70 \%$ of workers are women [6]. Thus, the vast majority of workers in the health sector are women, who experience a particular double burden of combining domestic responsibilities and the specific challenges of their working environment.

In the context of the health care system, the issue of reconciling family and paid work life is important because questions of the quality of health care are affected at different levels. This concerns, among other things, the specific forms of requirements and burdens of the professions working in the health care system, such as shift work, the individual satisfaction and performance of doctors, professional nurses, assistant professions, or therapeutic professions (e.g., physiotherapy, occupational therapy, psychotherapy) or also the guarantee of sufficient (medical) care density in rural areas depending on the availability of a family-friendly infrastructure. Studies from Australia, France, Malaysia, the Netherlands and Hungary show that among physicians of different specialties, the experience of poor work-life balance is positively associated with burnout, but also correlates with other unfavorable outcomes such as willingness to quit or impairment of subjective health [7]. International studies have shown that for professional nurses, conflicts over the balance of paid work and family life are associated with a stronger negative mood, emotional exhaustion, the intention to quit or change jobs, but also with worsening health parameters (pain symptoms, increased cardiometabolic risk score) [7]. In a survey of 120 physicians, a group of researchers around Jerg-Bretzke (2018) revealed that $97.4 \%$ would like to be able to take a break from paid work in case of a family emergency, part-time arrangements, $(96.6 \%)$ and emergency childcare $(90.4 \%)$. In addition, the doctors surveyed demanded that their executives provide more support of compatibility issues $(94.0 \%)$ [8].

In questions of the compatibility of family, care, and paid work, it is possible to draw on already well-developed social research. In addition to general brochures and official forms, there are numerous rigid information portals (e.g., https://www.bmfsfj.de) and websites (e.g., https://care.com) dedicated to solving common practical cases. They provide decision support to people in need of care, employees, social workers, and consultants and can be used for systematization, categorization, and solution assignment. However, the systematization itself as well as the formulation of low-threshold accessible answers must be implemented in the future.

\section{RELATED WORK}

The techniques described in the following section are the basis for the aggregation of system components presented in the main part of this work.

\section{A. Artificial intelligence}

Artificial intelligence comprises a broad portfolio of methods such as knowledge representation, reasoning, inference, problem strategies, expert and consulting support systems with explanatory components, speech comprehension, and machine learning. While symbolic AI dominated in the 1970s until the turn of the millennium, and the breakthrough of agentbased AI models in the 1990s opened up access to "autonomisation" and thus also to intelligent human-technology interaction for all kinds of application systems, classifiers with deep learning capabilities have recently been in the foreground. Particular strengths are attributed to symbolic AI, especially with regard to the reusability of knowledge models and the traceability of derived results. Of particular importance is the AI's experience with the possibilities and, above all, the limitations of modelling and concluding evaluation of vague, uncertain, incomplete, erroneous, and missing knowledge [9]. Machine learning enables technical systems to acquire knowledge independently, to adapt existing knowledge to new experiences, and thus to adapt independently and highly flexibly to changing situational requirements, such as changes in human interaction behaviour caused by experience learning. Due to these advantages, the use of AI is also a decisive guide for the economy and administration. Central to pattern recognition methods is data pre-processing and integration by means of AI-based identification of relevant features through feature engineering. Current methods for this are, for example, deep learning based autoencoders, which train neural networks in such a way that the attributes and elements that have the greatest possible influence on the creation of the knowledge model are identified [10], [11]. A second focus of problem solving with AI is reasoning and the necessary problem solving strategies [12], [13], [14]. Further topics of current AI research are the representation of high-dimensional data [15], [16] or projection methods [17], [18] or the application possibilities of different types of neural networks [19], [20], 
[21]. The processing of natural language, e.g., in chatbots, is a necessary technique at the forefront of AI innovations.

\section{B. Text Classification}

The text classification of legally relevant passages in brochures, forms, guidebooks, and web content can be carried out using automated procedures. Up to now, Support Vector Machines have been used, whose performance was superior to other methods in this task [22]. Recent studies have shown neural networks to be promising and state of the art. Numerous development steps have made it possible to make them available for a broad field of application [23], [24]. In addition, language models have recently been presented that have consistently improved the performance of neural classification tasks [25], [26]. The implementation of the intended automatic annotation of action guidelines for the classification of legally relevant text passages seems to be very promising.

\section{AI and application systems in the context of social services}

Chatbots act as virtual assistants, which are text-based dialog systems capable of mediating interaction between humans and machines in natural language and be equipped with the following features [27]:

1) filtering out superfluous information e.g., fillers, pauses, false starts;

2) determining an appropriate system response and the need for sophisticated decision making mechanism;

3) answer aggregation to present a summary answer rather than a just ranked list of results;

4) conversation management that considers and maintains the search goals, conversational history, and current state of the agent's understanding;

5) general knowledge that the agent should have about external world in order to efficiently exploit contextual information and correctly process the user's query;

6) and personality and moral responsibility to respond to emotional and sensitive queries.

A distinction can be made between rule-based chatbots and AI-based chatbots, which enable independent learning [1]. The continuous research and development of chatbots already enables their everyday use in many areas, e.g., in customer communication in e-commerce [28] or the financial sector [29]. In contrast, AI-based consulting on social services has not yet been researched [30].

\section{Regulatory hurdles of AI deployment}

Little research exists on the framework conditions for the use of an AI-based information application on legal issues. On the one hand, the particular ethical, legal, and social aspects of the new technology need to be worked out. The duties of care in programming, training, and use of machine learning systems have only been partially considered [31]. The High Level Expert Group on Artificial Intelligence (HEG-AI) of the EU Commission, which recently drafted guidelines, is investigating the framework conditions for an ethically and legally acceptable use of artificial intelligence [32]. Individual case studies on privacy and data protection aspects can only be found in blog posts by data protectionists [33].

\section{REFERENCE MODEL "PFADI.AI"}

The creation of the reference architecture in this section is the main contribution to this work. By combining proven methods and technologies of data processing, a high-level reference model, the information flows, and the technical layer of the bot are modelled and presented. Therefore the presented BERT technology serves as an enabler for the described framework.

\section{A. Methodology}

The main goal of this work relies on defining an architecture of a system that allows the integration of an AI-based chatbotsystem. For this purpose the authors propose a three-tier information system landscape "PFaDi.AI" which is based on well-proven artefacts. The presented work is based on the aggregation and combination of existing system artefacts to a new reference architecture. The creation of the model is based on the design techniques introduced by vom Brocke [34]. On a first higher layer the construction of the new architecture is presented in the next section. The following sections describe the connections of the used systems and their new arrangement to each other. Building blocks of these systems are described on a subordinate layer.

\section{B. PFaDi.AI}

The overall procedure shown in Fig. 1 of providing the information a user is searching for is described in this section. The detailed procedures of integrated components are described more precisely in the following sections. The first step in Fig. 1 starts with the initial interaction of the user with the PFaDi.AI-System. The first request is answered by the dialog management of PFaDi.AI and shows readiness for providing information. Further question and answer communication supports the definition of the topic and identifies the layer of the query space to be addressed. This is represented by steps $1-3$. Subsequently, the identified query space is used to prepare the search in the data ETL stack with the help of the BERT within the Information Retrieval. The aim is the semantic enrichment of the corpus transferred, in order to search the relevant data sinks in the following step 5 using the prepared keywords.

Following this, the identified structured or unstructured text fragments from the data sinks are prepared and broken down into processable sequences using topic modelling methods. After extracting the information from the text sequences with the help of qualifier algorithms, these sequences are brought back to Dialog Management in step 7 and made available to the user in step 8.

\section{Dialogue Management}

Dialogue Management determines the actual context of the dialogue. With respect to the features mentioned above to a 


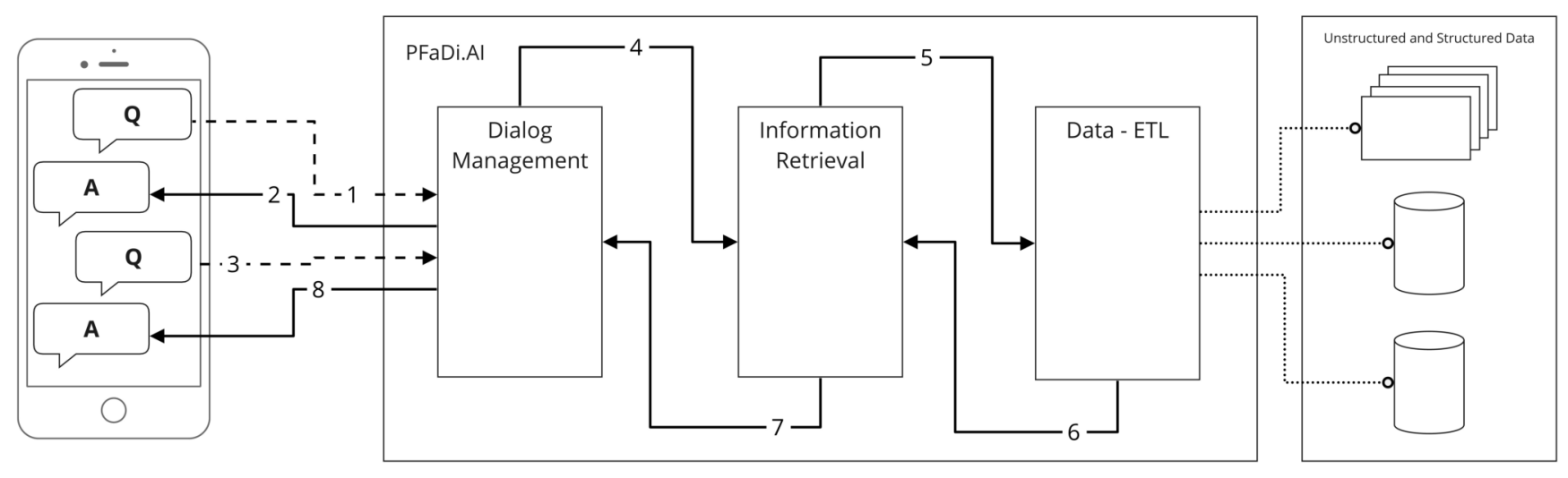

Fig. 1. High-Level Representation of the proposed architecture [own illustration]

chatbot, an architecture for an application system for social services was designed. For example, the user could say or write: "I need childcare for this week" and the bot would try to find appropriate service offerings. Then the user might add: "for the older of my two children," here the user refers to a part of the conversation that took place earlier. The bot has to correctly interpret before confirming with the user. Besides the general language, dialogue entry and exit play a special role in the conversation. When the bot starts the conversation proactively, the area of competence must be clearly defined. The definition of the dialogue exit can look manifold. It can range from pure information about the subject area of care or social services, direct booking of a service to preparing an appointment with a human consultant. An overview of the objectives of individual thematic sub-bots used in the proposed architecture is given. Based on the needs of the user, appropriate sub-bots are selected to generate high quality responses (see Table I). The individual bots are based on information transmitted by the user during the course of the conversation. The sub-bots are characterized by special domain knowledge (e.g., taxonomies and ontologies for care, paid work, and families).

TABLE I

DEFINING CHARACTERISTICS OF SUB-BOTS

\begin{tabular}{|l|l|}
\hline Name & Description \\
\hline Small Talk Bot & $\begin{array}{l}\text { By filling the bot's architecture with answers to } \\
\text { social inputs like "How Are You?," it is not only } \\
\text { prevented unnecessary fallbacks. The bot is strength- } \\
\text { ened with human qualities, which builds an invalu- } \\
\text { able connection with the user. }\end{array}$ \\
\hline Work Bot & $\begin{array}{l}\text { Answers all inquiries and provides information on } \\
\text { organizational issues within a company (e.g., swap- } \\
\text { ping shifts) as well as questions on employee rights. }\end{array}$ \\
\hline Health Care Bot & $\begin{array}{l}\text { Makes offers of care counselling and provides ap- } \\
\text { propriate information on the situation of affected } \\
\text { persons and relatives, finds services for the imple- } \\
\text { mentation of care services and gives advice when } \\
\text { applying for state support }\end{array}$ \\
\hline Family Bot & $\begin{array}{l}\text { Searches for offers of family related services. These } \\
\text { can be, for example, child care, animal care, do- } \\
\text { mestic help. An automatic booking of these services } \\
\text { should be possible. }\end{array}$ \\
\hline
\end{tabular}

The information flow of the chatbot-system is represented in Fig. 2. Users can chat with the bot not only via free text input but also speech-controlled. This is done via low-threshold use of common end devices. It's also possible to guide users through the dialogue with the help of buttons and ready-made story lines. This prevents questions being formulated in a way that is incomprehensible to the bot. No matter how well the bot is planned, it could always happen that it cannot answer a question. In this critical case, there are various escalation options: For example, a consultant can step in and take over the chat, the user can be referred to the telephone service, fill out a contact form or receive helpful documents for download. This should not result in any additional effort, otherwise the bot will not be perceived as a helper. In order to ensure targeted communication and avoid escalations the dialogue management must have mechanisms for processing feedback and policy learning. On the one hand, the agent takes the user's feedback to determine whether the bot is coping well with the conversation and whether the user is satisfied with the bot's responses. This encourages the bot to learn from mistakes and correct itself in future conversations. Through policy learning, the bot can be taught a positive language. The goal is to improve the overall satisfaction of the end user.

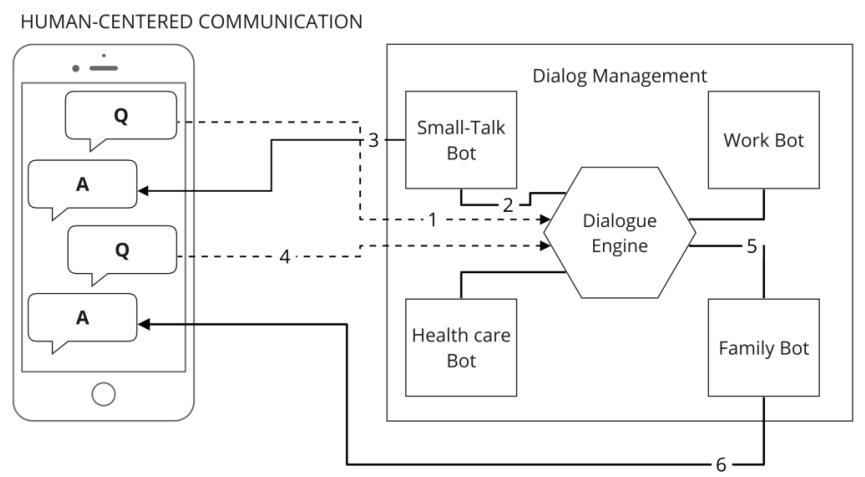

Fig. 2. Communication between human and chatbot [own illustration] 
Thus a network of successful paths has been derived and the bot is able to conduct the conversation to the satisfaction of the end user. The bot tries to learn from the interaction and to follow in detail the course of the conversation it had with similar users in the past. The technique of reinforcement learning can be used for this.

\section{Information Retrievel}

The system is placed in the area of information retrieval prepare information for further processing in subsequent systems in order to provide purposive information. Two information directions are addressed. On the one hand, the transferred question-answer constructs of the chatbots are prepared by an intent clearifier and then semantically processed by the BERT algorithm. On the other hand, sequences of answers are cut from identified relevant documents from the ELK-Stack for the transmission to the chatbot-system.

1) BERT: In the model, the BERT algorithm performs the task of providing the actual answer to the user's question. For this purpose, the user receives a content-related pre-selected sequence from the ELK, which is then searched and evaluated for the detailed answer to the user's question. As BERT is one of the core components of the system, it is discussed in more detail in chapter V. Here the technical aspects of BERT, such as the input / output representation of questions and answers or the pre-training and fine-tuning of BERT, will be discussed.

2) Intent Clearifier: The recognition of the intention of the questioner is helpful for the effective generation of an appropriate answer. For this purpose, Language Understanding Tools such as LUIS can be used. Fig. 3 shows an example of how to determine the probability of an intent to a user's question:

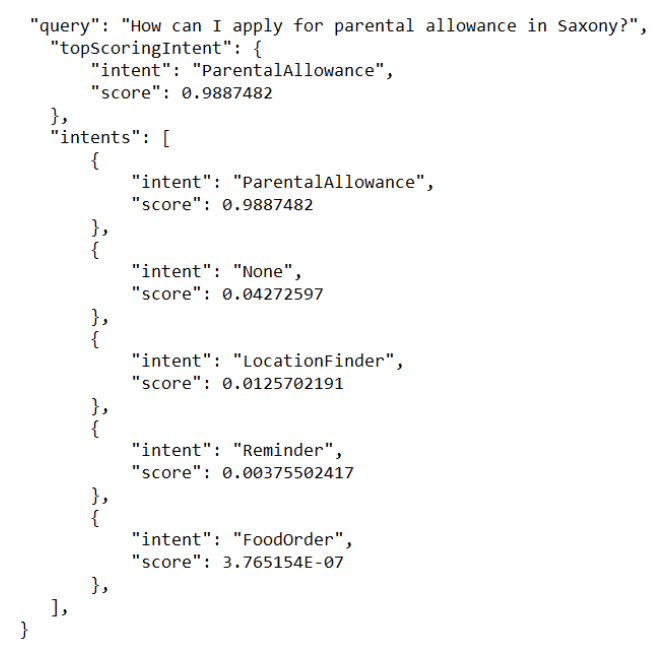

Fig. 3. Intent Clearification [own illustration]

3) Topic Modelling: In the model, communication with the user is controlled on the basis of a question and answer qualifier. This is intended to ensure that the question qualifier has all the information needed to answer the question. Here, sufficient keywords must be available to delimit the content of the topic area. On the other hand, the answer qualifier is intended to ensure that the user is only provided with information in the chatbot that exceeds at least one threshold value in the probability of correctly answering his question. Within the presented architecture, a topic modelling framework performs the task of deriving the required information from the information sinks identified by the crawler. For this purpose, the entire web page stored in the ELK stack is first segregated into paragraphs using a topic-modeling procedure. Afterwards, the paragraphs with the highest similarity to the question are made available for answering to the corresponding bot.

\section{E. Data ETL}

During the data ETL step, the contents prepared by BERT are passed to the ELK stack. Within the stack, available blocks of information are searched for on the basis of the identified subject areas and passed to the information retrieval. The information blocks are cyclically updated by a crawler ecosystem and stored in the ELK stack as shown in Fig. 4. While step 3 is triggered per each request, steps 6 and 7 are performed periodically.

1) Crawler Ecosystem: A web crawler is used to programmatically go over a selection of websites and extract its data. Such a crawler allows you to create a large text corpus from selected web pages. For the purposes of this work, the official websites on reconciling family and paid work at EU level and at federal and state level will be searched in German language (e.g., https://www.bmfsfj.de/). For a corresponding specialisation of the information, additional company-specific information can be added to the text corpus.

As an application framework scrappy is used to crawl these websites for structured (e.g., HTML) and unstructured data (e.g., PDF). You can get the HTML source code through the scrappy downloader.

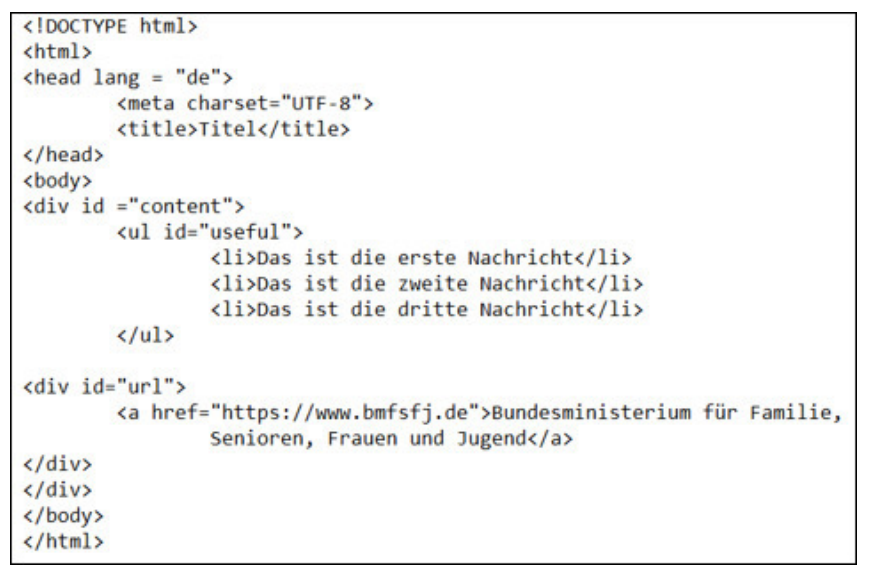

Fig. 5. HTML source code of a simple web page [own illustration]

The scrappy downloader can access the web data according to the specified URL. With selectors certain parts of the HTML document can be specified by Xpath expressions. For the 


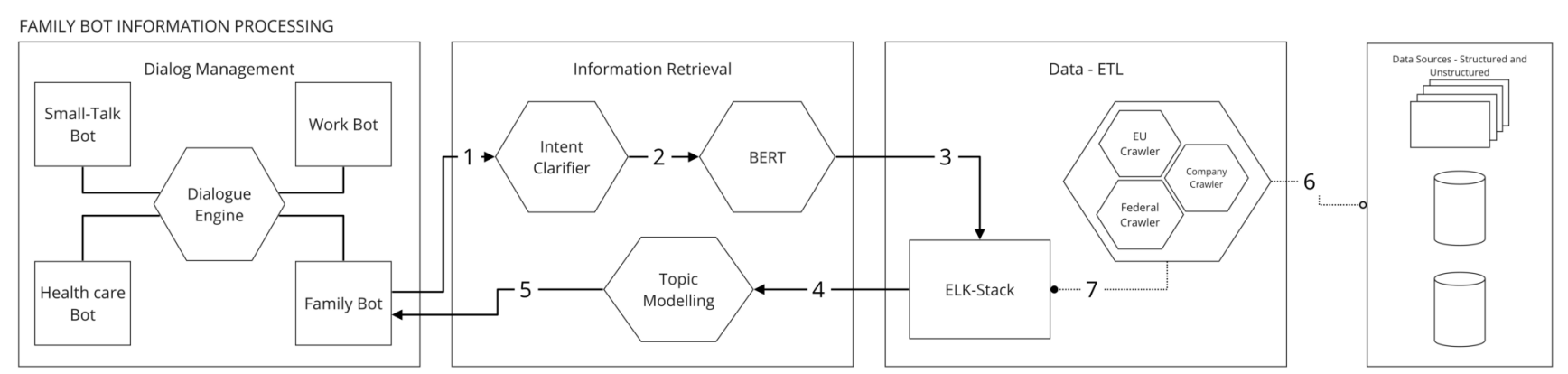

Fig. 4. Information flow — technical layer with communication between system aggregates [own illustration]

HTML source code in Fig. 5 you can construct an Xpath for selecting the text under the label and extracting the text inside the attribute from the HTML source code.

2) ELK-Stack: This ELK stack is used to collect and store the structured and unstructured data generated by the web crawler. Furthermore, the document-oriented database Elasticsearch is used to retrieve the collected information for the purpose of finding an appropriate response. The Elastic or "ELK" stack contains three open source tools: Elasticsearch, Logstash, and Kibana. It is a document-oriented database designed to store, retrieve, and manage document-oriented or semi-structured data. Logstash is a server-side data processing pipeline that simultaneously ingests data from multiple sources, converts it and then sends it to a storage location, such as Elasticsearch. Kibana allows users to visualize data through charts and tables in Elasticsearch.

\section{BERT: BIDIRECTIONAL ENCODER REPRESENTATIONS FROM TRANSFORMERS}

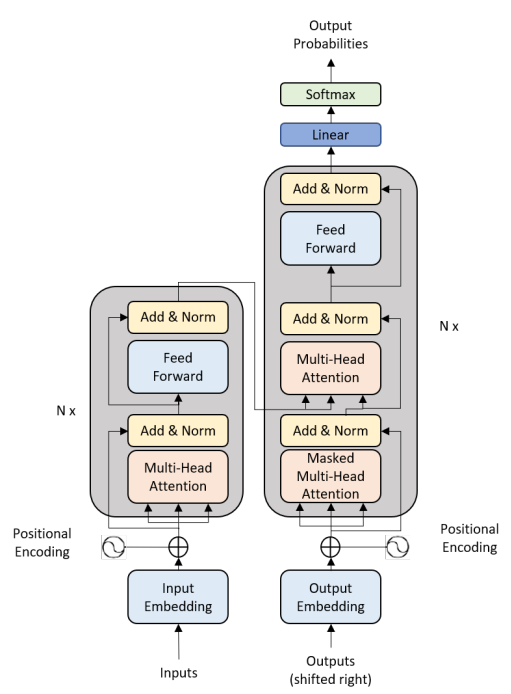

Fig. 6. Transformer Model Architecture [35]

Bidirectional Encoder Representations from Transformers (BERT) [36] represents the state of the art of language models that pretrain deep bidirectional representations for a variety of tasks. Possible fields of application include text classification, named entity recognition or question answering tasks (i. e. SQUAD 2.0) [37]. The Bert implementation is a two-stage process consisting of a pre-training step and a fine-tuning step. During the pre-training step the model is trained on unlabelled data. Based on this the fine-tuning step follows. Here the model is initialized with the pre-trained parameters, which are then fine-tuned with labelled data from downstream tasks.

\section{A. BERT Baseline Model Architecture}

BERT is based on the transformer architecture described by Vaswani et al. [35]. Fig. 6 shows a transformer model architecture. For a more intensive study of Transformer Architecture, please refer to [35].

\section{B. Input / Output Representations}

The input representation of BERT can represent both a single sentence and a pair of sentences (e.g. <Question, Answer $>$ ) in one token sequence. An example sentence could be as follows:

\section{Where can I find the nearest childcare?}

The tokinization of this sentence is then as follows:

['[CLS]', 'Where', 'can', 'I', 'find', 'the', 'nearest', 'childcare', '?', '[SEP]']

The first token of each sequence is always a special class token 'CLS'. The hidden state associated with this token is used for classification tasks as an aggregated sequence representation. For a pair of sentences within a sequence another special token 'SEP' is used. This token is used to separate the individual sentences. For a question-answer task the following structure can be used:

['CLS', ' $q w 1$ ', ... ' $q w N$ ', 'SEP', 'cwl', . . . ., 'c $c w M$ ', 'SEP']

It can be used this input representation for each sequence. Here 'CLS' stands for a classification token and 'SEP' for a separation token. Furthermore 'qw' represent a question word 
with $\mathrm{N}$, the number of question words and ' $\mathrm{cw}$ ' represents a context word with $\mathrm{M}$, the number of context words. The representations are taken by the first encoder transformer and retrieve embeddings for each of the words. The input embeddings are the sum of the token embeddings, the segmentation embeddings, and the position embeddings.

\section{PRE-TRAINING BERT}

Instead of using traditional left-to-right or right-to-left language models [38][39], it can be used as an unsupervised tasks to pre-train BERT. For the purpose of Question Answering and Natural Language Inference the Next Sentence Prediction task is described in this section.

\section{A. Next Sentence Prediction}

For downstream tasks like Question Answering (QA) it is important to understand the relationships between two sentences, which is not directly captured by language models. For that reason BERT is pre-trained for a binarized next sentence prediction task. This task can be generated from any monolingual corpus. $50 \%$ of the time $\mathrm{B}$ is the actual next sentence that follows A (labeled as isNext) when choosing the sentences A and B for each pre-training example. For the other $50 \%$ it is a random sentence from the corpus (labeled as NotNext). The following example illustrates the next sentence prediction task:

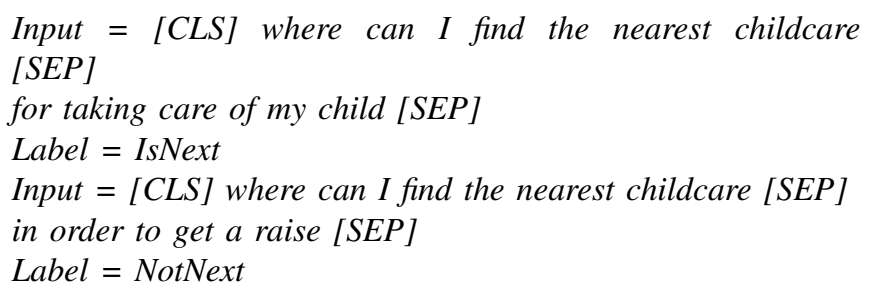

The next sentence prediction task is related to representation learning objectives used in [40] and [41].

\section{B. Pre-training and fine-tuning BERT}

For pre-training purposes the corpus data in German language is used, which was created from the text data obtained by the web crawler.

BERT uses the self-attention mechanism to unify the encoding of the text pairs as well as the application of the bidirectional cross attention between concatenated sentences. Because of that fine-tuning is a straightforward task. For fine-tuning the task-specific inputs and outputs are simply plugged into BERT and an end-to-end fine-tuning of all parameters is performed. At the input, sentence $\mathrm{A}$ and sentence $\mathrm{B}$ from pre-training are analogous to question-passage pairs in question answering. At the output, the token representations are fed into an output layer for token level tasks, e.g., question answering, and the [CLS] representation is fed into an output layer for classification. The hyperparameter-tuning includes setting an appropriate batch-size, learning rate or number of epochs and is very task specific.

\section{CONCLUSiOn AND Future WORK}

In this work a conception of an AI based assistant to support the compatibility of family and paid work was presented. For this purpose, the essential components of the bot from data generation, data storage, and data flows to information retrieval and data management were described. The main component of the system is represented by BERT, which contributes to the classification of user questions in terms of content and thus to a more optimised finding of answers by the sub-bots. The presented architecture components are state of the art components from the areas of data pipelines, data processing, and artificial intelligence which have been aggregated and combined into a new reference architecture and thus answer the research question posed at the beginning about the appearance of a suitable approach for a chatbot architecture for a better compatibility of family and paid work. This presented preliminary approach for a chatbot architecture for better reconciliation of family and paid work, especially in the care and health sector, is a promising starting point for further developments. The bot is supposed to be used by the user via specific end devices (e.g., smart phone, tablet, pc) based on speech or text. Whereby the use should be as low-threshold as possible. In a next step, the reference model and its components will be implemented in a first prototype with the help of the latest technologies from science and engineering. This is to be used initially as a prototype and then productively in the above-mentioned areas in order to mitigate the effects of the corona pandemic on the compatibility of family and paid work and to better master future challenges. By integrating PFaDi.AI into the PFaDi platform, the aim is to enable employees to structure their daily lives efficiently through easily accessible information and services and to minimise the impact of the corona pandemic on the reconciliation of paid work and family life. For this reason it is necessary to implement the described steps as quickly as possible and to present them to the scientific community in order to discuss the presented approach and raise additional potential.

\section{REFERENCES}

[1] H. N. Io and C. B. Lee, "Chatbots and conversational agents: A bibliometric analysis," in 2017 IEEE International Conference on Industrial Engineering and Engineering Management (IEEM), 2017, pp. 215-219.

[2] P. Böttinger and J. zu Putlitz, Die Zukunft der Medizin: Disruptive Innovationen revolutionieren Medizin und Gesundheit. Mit einem Geleitwort von Hasso Plattner. Berlin: MWV Medizinisch Wiss. Ver, 2019. [Online]. Available: https://books.google.de/books?id=42rKDwAAQBAJ

[3] F. Resch and P. Parzer, Entwicklungspsychopathologie und Psychotherapie - Kybernetische Modelle zur funktionellen Diagnostik bei Jugendlichen. Berlin Heidelberg New York: Springer-Verlag, 2015.

[4] S. Kaiser and M. J. Ringlstetter, Work-Life Balance - Erfolgversprechende Konzepte und Instrumente für Extremjobber. Berlin Heidelberg New York: Springer-Verlag, 2010.

[5] S. Peters and J. v. Garrel, Arbeits - Zeitsouveränität für Führungskräfte von Morgen - Vereinbarkeit von Beruf und Privatheit. München: Rainer Hampp Verlag, 2013.

[6] M. Boniol, M. McIsaac, L. Xu, T. Wuliji, D. K., and J. Campbell, "Gender equity in the health workforce: Analysis of 104 countries." Health Workforce Working paper 1., 2019. 
[Online]. Available: https://apps.who.int/iris/bitstream/handle/10665/ 311314/WHO-HIS-HWF-Gender-WP1-2019.1-eng.pdf?ua=1

[7] M. Lukasczik et al., Compatibility of Work and Family Life of Employees in the Healthcare Sector: An Issue in Health Services Research. Gesundheitswesen (Bundesverband der Ärzte des Öffentlichen Gesundheitsdienstes (Germany), 2013.

[8] K. P. Jerg-Bretzke, L., H. C. Traue, and K. Limbrecht-Ecklundt, "What you will": Results of an Empirical Analysis of the Need to Improve Work-life Balance for Physicians. Berlin: MWV Medizinisch Wiss. Ver, 2018.

[9] S. Russell and P. Norvig, Artificial Intelligence: A Modern Approach, Global Edition. CL, may 2019. [Online]. Available: https://www.xarg. org/ref/a/1292153962/

[10] T. Nolle, S. Luettgen, A. Seeliger, and M. Mühlhäuser, "Analyzing business process anomalies using autoencoders," Mach. Learn., vol. 107, no. 11, p. 1875-1893, Nov. 2018. [Online]. Available: https://doi.org/10.1007/s10994-018-5702-8

[11] C. Zhou and R. C. Paffenroth, "Anomaly detection with robust deep autoencoders," in Proceedings of the 23rd ACM SIGKDD International Conference on Knowledge Discovery and Data Mining, ser. KDD '17. New York, NY, USA: Association for Computing Machinery, 2017, p. 665-674. [Online]. Available: https://doi.org/10.1145/3097983.3098052

[12] L. Barreto and E. Mota, "Self-organized inductive reasoning with nemus," CoRR, vol. abs/1906.06761, 2019. [Online]. Available: http://arxiv.org/abs/1906.06761

[13] Y. Jiang and M. Bansal, "Avoiding reasoning shortcuts: Adversarial evaluation, training, and model development for multi-hop QA," CoRR, vol. abs/1906.07132, 2019. [Online]. Available: http://arxiv.org/abs/ 1906.07132

[14] M. Qu and J. Tang, "Probabilistic logic neural networks for reasoning," CoRR, vol. abs/1906.08495, 2019. [Online]. Available: http://arxiv.org/abs/1906.08495

[15] S. Liu, D. Maljovec, B. Wang, P. Bremer, and V. Pascucci, "Visualizing high-dimensional data: Advances in the past decade," IEEE Transactions on Visualization and Computer Graphics, vol. 23, no. 3, pp. 1249-1268, 2017.

[16] C. Seifert et al., Visualizations of Deep Neural Networks in Computer Vision: A Survey, ser. Studies in Big Data. Springer, 2017, pp. 123-144.

[17] P. Oesterling, C. Heine, H. Janicke, G. Scheuermann, and G. Heyer, "Visualization of high-dimensional point clouds using their density distribution's topology," IEEE Transactions on Visualization and Computer Graphics, vol. 17, no. 11, pp. 1547-1559, 2011.

[18] C. Heine et al., "A survey of topology-based methods in visualization," Comput. Graph. Forum, vol. 35, no. 3, p. 643-667, Jun. 2016.

[19] J. Wang, L. Gou, H. Shen, and H. Yang, "Dqnviz: A visual analytics approach to understand deep q-networks," IEEE Transactions on Visualization and Computer Graphics, vol. 25, no. 1, pp. 288-298, 2019.

[20] B. C. Kwon et al., "Retainvis: Visual analytics with interpretable and interactive recurrent neural networks on electronic medical records," CoRR, vol. abs/1805.10724, 2018. [Online]. Available: http://arxiv.org/abs/1805.10724

[21] V. Dibia and C.. Demiralp, "Data2vis: Automatic generation of data visualizations using sequence to sequence recurrent neural networks," CoRR, vol. abs/1804.03126, 2018. [Online]. Available: http://arxiv.org/abs/1804.03126

[22] A. Niekler et al., "ILCM - a virtual research infrastructure for largescale qualitative data," in Proceedings of the Eleventh International Conference on Language Resources and Evaluation (LREC 2018). Miyazaki, Japan: European Language Resources Association (ELRA) May 2018. [Online]. Available: https://www.aclweb.org/anthology/ L18-1209

[23] A. Jacovi, O. Sar Shalom, and Y. Goldberg, "Understanding convolutional neural networks for text classification," in Proceedings of the 2018 EMNLP Workshop BlackboxNLP: Analyzing and Interpreting
Neural Networks for NLP. Brussels, Belgium: Association for Computational Linguistics, Nov. 2018, pp. 56-65. [Online]. Available: https://www.aclweb.org/anthology/W18-5408

[24] S. Lai, L. Xu, K. Liu, and J. Zhao, "Recurrent convolutional neural networks for text classification," in AAAI, 2015.

[25] J. Devlin, M. Chang, K. Lee, and K. Toutanova, "BERT: pre-training of deep bidirectional transformers for language understanding," CoRR, vol. abs/1810.04805, 2018. [Online]. Available: http://arxiv.org/abs/ 1810.04805

[26] J. Li, A. Sun, J. Han, and C. Li, "A survey on deep learning for named entity recognition," CoRR, vol. abs/1812.09449, 2018. [Online]. Available: http://arxiv.org/abs/1812.09449

[27] L. Azzopardi, M. Dubiel, M. Halvey, and J. Dalton, "Conceptualizing agent-human interactions during the conversational search process," 2018.

[28] L. Cui, S. Huang, F. Wei, C. Tan, C. Duan, and M. Zhou, "SuperAgent: A customer service chatbot for e-commerce websites," in Proceedings of ACL 2017, System Demonstrations. Vancouver, Canada: Association for Computational Linguistics, Jul. 2017, pp. 97-102. [Online]. Available: https://www.aclweb.org/anthology/P17-4017

[29] T. Okuda and S. Shoda, "Ai-based chatbot service for financial industry," Fujitsu Scientific and Technical Journal, vol. 54, no. 2, pp. 4-8, 2018.

[30] E. H. Almansor and F. K. Hussain, "Survey on intelligent chatbots: State-of-the-art and future research directions," in CISIS, 2019.

[31] N. Bilski and T. Schmid, "Verantwortungsfindung beim einsatz maschinell lernender systeme, njoz 2019, 657-661," vol. 19, p. 657, 052019.

[32] B. J. Wagner, "Rechtliche Analyse der Kommunikationsströme zwischen Assistenzsystemen," in Konnektivität von Assistenzsystemen: Eine vertrags- und datenschutzrechtliche Analyse kollaborativer Softwareagenten im Internet of Things. Wiesbaden: Springer Fachmedien Wiesbaden, 2020, pp. 65-436. [Online]. Available: https://doi.org/10.1007/978-3-658-29628-5_3

[33] H. Harkous, K. Fawaz, K. G. Shin, and K. Aberer, "Pribots: Conversational privacy with chatbots," in Twelfth Symposium on Usable Privacy and Security (\{SOUPS\} 2016), 2016.

[34] J. v. Brocke, Konstruktionstechniken zur Referenzmodellierung - Enzyklopaedie der Wirtschaftsinformatik, 012018.

[35] A. Vaswani et al., "Attention is all you need," CoRR, vol. abs/1706.03762, 2017. [Online]. Available: http://arxiv.org/abs/1706 03762

[36] J. Devlin, M. W. Chang, K. Lee, and K. Toutanova, "BERT: pre-training of deep bidirectional transformers for language understanding," CoRR, vol. abs/1810.04805, 2018. [Online]. Available: http://arxiv.org/abs/ 1810.04805

[37] D. Takeuchi and K. Tran, "Improving squad 2.0 performance using bert + + ," 32nd Conference on Neural Information Processing Systems (NIPS), vol. abs/1810.04805, 2018. [Online]. Available: http://arxiv.org/abs/1810.04805

[38] M. E. Peters et al., "Deep contextualized word representations," CoRR, vol. abs/1802.05365, 2018. [Online]. Available: http://arxiv.org/abs/ 1802.05365

[39] A. Radford, K. Narasimhan, T. Salimans, and I. Sutskever, "Improving language understanding by generative pre-training," https://cdn.openai.com/research-covers/language-unsupervised/ language_understanding_paper.pdf, 2018.

[40] Y. Jernite, S. R. Bowman, and D. A. Sontag, "Discourse-based objectives for fast unsupervised sentence representation learning," CoRR, vol. abs/1705.00557, 2017. [Online]. Available: http://arxiv.org/ abs/1705.00557

[41] L. Logeswaran and H. Lee, "An efficient framework for learning sentence representations," CoRR, vol. abs/1803.02893, 2018. [Online]. Available: http://arxiv.org/abs/1803.02893 


\section{Joint $40^{\text {th }}$ IEEE Software Engineering Workshop and 7th International Workshop on Cyber-Physical Systems}

T HE IEEE Software Engineering Workshop (SEW) is the oldest Software Engineering event in the world, dating back to 1969. The workshop was originally run as the NASA Software Engineering Workshop and focused on software engineering issues relevant to NASA and the space industry. After the $25^{\text {th }}$ edition, it became the NASA/IEEE Software Engineering Workshop and expanded its remit to address many more areas of software engineering with emphasis on practical issues, industrial experience and case studies in addition to traditional technical papers. Since its $31^{\text {st }}$ edition, it has been sponsored by IEEE and has continued to broaden its areas of interest.

One such extremely hot new area are Cyber-physical Systems (CPS), which encompass the investigation of approaches related to the development and use of modern software systems interfacing with real world and controlling their surroundings. CPS are physical and engineering systems closely integrated with their typically networked environment. Modern airplanes, automobiles, or medical devices are practically networks of computers. Sensors, robots, and intelligent devices are abundant. Human life depends on them. CPS systems transform how people interact with the physical world just like the Internet transformed how people interact with one another.

The joint workshop aims to bring together all those researchers with an interest in software engineering, both with CPS and broader focus. Traditionally, these workshops attract industrial and government practitioners and academics pursuing the advancement of software engineering principles, techniques and practices. This joint edition will also provide a forum for reporting on past experiences, for describing new and emerging results and approaches, and for exchanging ideas on best practice and future directions.

\section{TOPICS}

The workshop aims to bring together all those with an interest in software engineering. Traditionally, the workshop attracts industrial and government practitioners and academics pursuing the advancement of software engineering principles, techniques and practice. The workshop provides a forum for reporting on past experiences, for describing new and emerging results and approaches, and for exchanging ideas on best practice and future directions.

Topics of interest include, but are not limited to:

- Experiments and experience reports
- Software quality assurance and metrics

- Formal methods and formal approaches to software development

- Software engineering processes and process improvement

- Agile and lean methods

- Requirements engineering

- Software architectures

- Design methodologies

- Validation and verification

- Software maintenance, reuse, and legacy systems

- Agent-based software systems

- Self-managing systems

- New approaches to software engineering (e.g., search based software engineering)

- Software engineering issues in cyber-physical systems

- Real-time software engineering

- Safety assurance \& certification

- Software security

- Embedded control systems and networks

- Software aspects of the Internet of Things

- Software engineering education, laboratories and pedagogy

- Software engineering for social media

\section{TECHNiCAL SESSION CHAIRS}

- Bowen, Jonathan, Museophile Ltd., United Kingdom

- Hinchey, Mike (Lead Chair), Lero-the Irish Software Engineering Research Centre, Ireland

- Szmuc, Tomasz, AGH University of Science and Technology, Poland

- Zalewski, Janusz, Florida Gulf Coast University, United States

\section{Program CommitTeE}

- Ait Ameur, Yamine, IRIT/INPT-ENSEEIHT, France

- Banach, Richard, University of Manchester, United Kingdom

- Challenger, Moharram

- Cicirelli, Franco, Universita della Calabria, Italy

- Ehrenberger, Wolfgang, Hochschule Fulda, Germany

- Gomes, Luis, Universidade Nova de Lisboa, Portugal

- Gracanin, Denis, Virginia Tech, United States 
- Havelund, Klaus, Jet Propulsion Laboratory, California Institute of Technology, United States

- Hsiao, Michael, Virginia Tech, United States

- Nesi, Paolo, DSI-DISIT, University of Florence, Italy

- Pullum, Laura, Oak Ridge National Laboratory, United States

- Sanden, Bo, Colorado Technical University, United States

- Sekerinski, Emil, McMaster University, Canada

- Sojka, Michal, Czech Technical University, Czech Re- public

- Trybus, Leszek, Rzeszow University of Technology, Poland

- van Katwijk, Jan, Delft University of Technology, The Netherlands

- Vardanega, Tullio, University of Padova, Italy

- Velev, Miroslav, Aries Design Automation, United States

- Zhu, Huibiao, Software Engineering Institute - East China Normal University 


\section{Capturing the Evolution of Service-oriented Systems with Architectural Decisions}

\author{
Szymon Kijas \\ Warsaw University of Technology \\ Warsaw 00-661, Poland \\ Email: szymon.kijas@pw.edu.pl
}

\author{
Andrzej Zalewski \\ Warsaw University of Technology \\ Warsaw 00-661, Poland \\ Email: a.zalewski@elka.pw.edu.pl
}

\begin{abstract}
Software evolution is becoming ever more important. SOA is nowadays a well-established and popular software technology. Because of its properties, such as loose-coupling between services and their reconfigurable composition, SOA is an architecture that is particularly suitable for rapidly evolving systems. However, the research on the evolution methodologies for SOA systems is rather scarce. We present a model called MAD4SOA, developed in order to support and capture the evolution of service-oriented systems. Architectural decisions are the first class entities that represent the evolution of a serviceoriented system. They are accompanied by a set of relations between model entities and formal integrity constraints. The suitability of the MAD4SOA model has been validated using the real-world example of a system operated in a clearing house company.
\end{abstract}

\section{INTRODUCTION}

$\mathbf{S}$ ERVICE-ORIENTED architectures assume that software systems should be built out of services that are looselycoupled (easily changeable!) and composable into reconfigurable business processes. This is a great support to systems evolvability. SOA is certainly one of the software technology answers to the rising speed of software evolution. It is now a well-established software technology. Although there are comprehensive methodologies specially crafted for development of SOA systems, RADM [6], Erl's approach [13], SOMA [14], SOMF [1], M. Papazoglou's methodology [15], the research record on the methods supporting the evolution of SOA-based systems is rather scarce. The research presented in this paper is aimed at filling this gap.

We developed a complete methodology, called MAD4SOA, for evolving SOA systems. It comprises: the evolution process, the model for capturing architectural decisions during the evolution, which includes the model of an evolving serviceoriented system, set of relations between model entities and integrity constraints. The course of the evolution is captured with architectural decisions, which are first-class entities in the proposed approach. It has been validated on a number of evolution steps of a real-world system operating in a clearing house company. Because of the space limitations, we present here the core concepts of the MAD4SOA methodology, namely, the architecture decision model, set of relations between model entities and its integrity rules. The model has been tailored to the specifics of software evolution - it contains Request for change, which is bound to all the important data describing a single evolution step (requirements, architectural decisions, versions of the SOA system and others). Using a significant fragment of a real-world example, we show that it goes well with the real conditions of service-oriented systems evolution.

The rest of the paper is organised as follows: Section II contains an analysis of related research; Section III presents the entire modelling approach, relations and integrity constraints, as well as the diagrammatical notation; and Section IV sets out a case study that presents the use of the model. Finally, in Section V, we discuss the proposed modelling approach against the related research. The conclusions and research outlook close the paper.

\section{RELATED WORK}

Software architecture is currently perceived as a result of a set of architectural decisions [3], [4], [5]. Architectural decisions can be documented in the form of text records [2], [3], [8], [11] or as diagrammatic models [12], [10]. The model of a single architectural decision usually includes a description of the problem, considered architectural solutions to this problem and their characteristics, an indication of the chosen solution, and the rationale for that choice.

Models of architectural decisions are usually accompanied by a set of relations provisioned for capturing the asscociations between architectural decisions - a number of such sets have been proposed so far [10], [6], [7], [8]. In the existing models, the decisions can also be linked to the engineering artifacts [17] enabling a variety of tracability options.

While the existing research focuses mainly on the modeling of architectural decisions, much less effort has been devoted to the evolution of architectures represented as a set of architectural decisions. The need for capturing the changes to architecture decisions has been indicated in [20]. A special view for architecting and evolving design decisions has been proposed in [16].

Apart from these general-purpose architecture decision models, RADM (Reusable Architectural Decision Model) has been proposed in [6]. It has been specially tailored to assist architecting of SOA systems. It combines architecture decisions with a set of relations, integrity constraints and some inference methods, as well as a means of classification of architectural decisions (levels and topic groups). It is also possible to link the architectural decisions with architectural elements [17]. 
However, RADM does not explicitly address the evolution of a SOA system. It does not allow for the capture of consecuitive steps of service-oriented architecture evolution.

An attempt to address the evolution of the decisions made during the evolution of service-oriented system has been undertaken in [18], however, the proposed solutions focus only on the decisions, which regard the composition of business processes out of services. SOAD framework [19], in turn, addresses the problem of reusing architectural decisions. There is also an extensive study on the choice between REST and SOAP webservices [21].

Outside the scope of research on architectural decisions, the topic of evolution of SOA systems has been rather scarcely addressed in the existing literature. Existing methodologies of SOA system's devlopment, such as Erl's approach [13], SOMA [14], SOMF [1], M. Papazoglou's methodology [15], address mainly the problem of early evolution of a SOA system (for example: bug fixing at an early stage of operation of the system) as well as aspects of small releases in the course of agile SOA system development [22].

This short survey leads to the conclusion that development of models and methodology supporting the evolution of SOA systems is an open research issue. Its resolution seems to be both important for software engineering practice as currently evolution is the main part of system lifecycle. It can also provide important insights into the intrinsic problems connected with applying the concepts of architecture decisionmaking in practice. In this paper we focus on the introduction and validation of a model suitable for capturing evolution of SOA systems, while combining a complete development methodology is supposed to be supplemented in later research.

\section{CAPturing the Evolution OF SERVice-ORIEnted SYSTEMS ARCHITECTURE}

In order to capture the evolution of service-oriented systems architecture, we developed a MAD4SOA model (Maps of Architectural Decisions for Service-Oriented Architecture). MAD4SOA comprises:

- the model of service-oriented system - it represents the main entities of a SOA system - section III.A

- the model of architectural decisions and diagrammatical notation that will be used in order to document the evolution of a service-oriented system - section III.B;

- the set of relations between the model's components (e.g. alternatives, decision problems) - section III.C,

- the fourteen integrity constraints (section III.D).

The MAD4SOA model combines a model of a SOA system with a model of architectural decisions. It extends our earlier model and diagrammatical notation named "Maps of Architectural Decisions" (MAD) presented in [10]. Its conceptual roots can also be traced back to RADM model by Zimmerman et al. [6] and Harrison et al. in a paper on architectural decisions [11].

\section{A. Model of a Service-Oriented System}

A SOA system model (Fig. 1) comprises three tiers: business processes, services and system components. It should be noted that they follow a scheme similar to the TOGAF [9] standard, though the user interface is not included in our model, as issues related to that are beyond the scope of our research. The model represents the following semantics:

- Business processes are composed of the Activities, which in turn are achieved by the Services. The latter are indicated by the realizes association between the Activity and Service classes in the UML model.

- The Services, in turn, are divided into three categories: 1) Complex services 2) Simple services and 3) Foreign services. A Complex service is a composition of other services, a Simple service is not a composition of other services and a Foreign service is a service delivered by an external provider.

- Components implement the functionalities of a Simple service and are indicated by the invokes association between a Simple Service and the Service Component classes.

- A Service Component may use an Operational component in order to implement its functionality. This is represented by the use association connecting a Service Component with the Operational Component class. The use of a database by a service component is a common example of this relation.

Although, the presented model captures the most important entities of the service-oriented system, it can still be expanded, depending on the needs of a given organisa-tion. Let us emphasise that the model does not assume any specific form of docu-mentation of the instances of its entities. This may be just an identifier of a given component in the ITSM register or a set of diagrams, text documents, formal models or even fragments of source code or reference to its repository. The service-oriented system model is used to represent releases of the system that result from the consecu-tive evolution steps compare section III.

\section{B. Model of Architectural Decisions}

The complete model designed to capture the evolution of service-oriented systems with architectural decisions is presented in Fig. 2. The meaning of the entities of the MAD4SOA model of architectural decisions can be explained as follows (italics indi-cate names of classes or associations in the model):

1) Request for change (RFC) class represents the typical document prepared in order to launch the modification process. It contains the specification of changes that should be implemented in a given evolution step. The RFC contains the list of architecturally significant requirements that must be met by the resolution of a number of decision problems. Each solution of such a problem defines the change being either the addition, removal or modification of at least one of the elements 


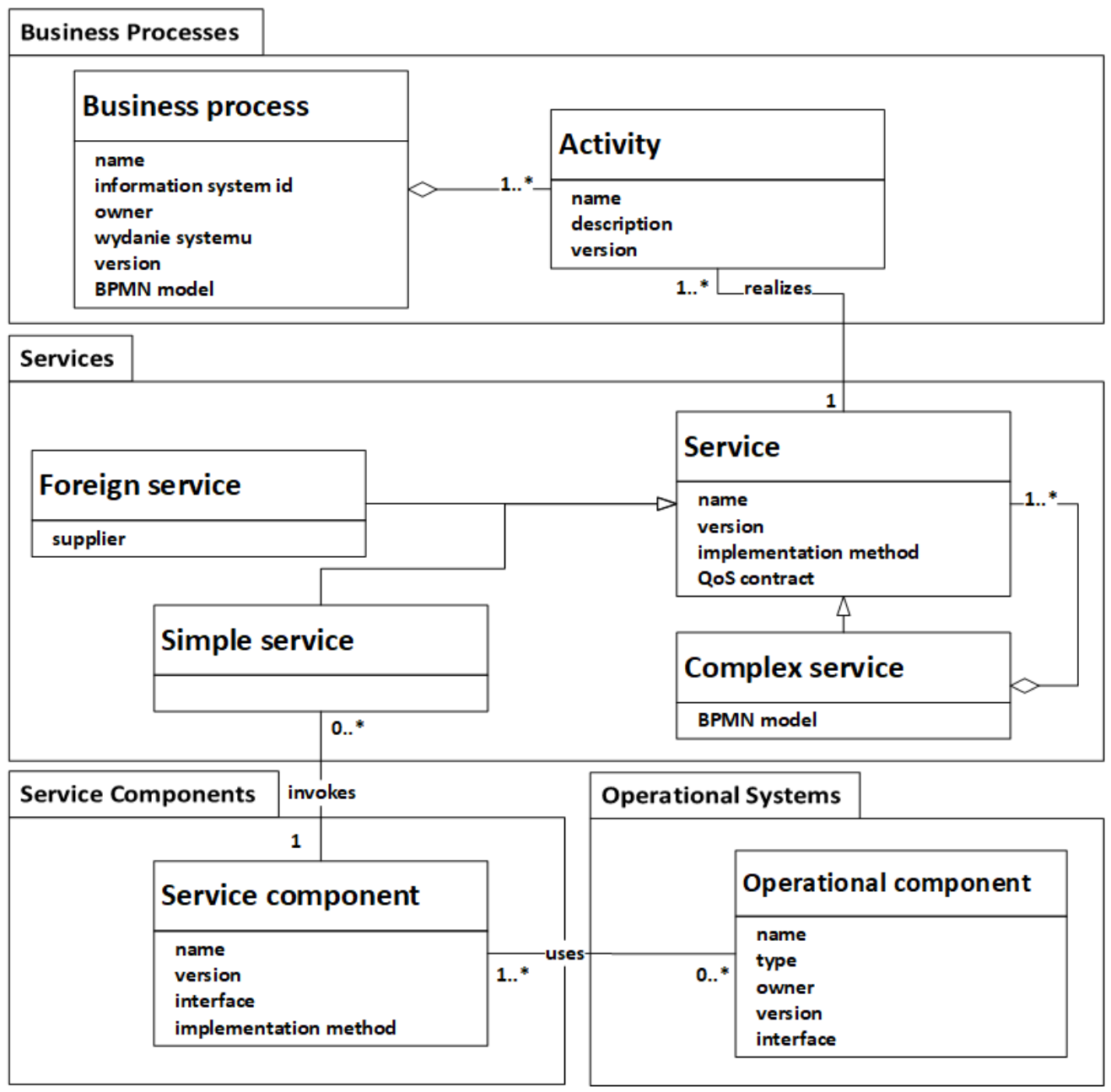

Fig. 1. The model of a service-oriented system.

of an instance of a SOA system model. Resolving all the problems makes a new system release. The current phase of work on the realisation of the request for change is represented by one of seven states that the Request for change could be in one of the following states: defined the request for change has been defined and it is waiting for the start of realisation, evaluated - the change has been assessed and it is waiting for approval, approved - the change has been approved and it is waiting for realisation, designed - the change design has been realised, implemented - the change has been implemented and deployed, realised (after review) - a review of the change has been carried out and the evolution step has been completed, cancelled - the change request has been cancelled (it was decided during the acceptance of the change that it will not be implemented).

2) The current release of the system is indicated by the association old binding the Request for change and Business process class.

3) Requirements relevant to a given Decision problem are distinguished by a motivates relation - compare section III.C. The requirement can be in one of the three states: defined - the requirement has been defined and it is awaiting for realisation, in progress - works are ongoing, completed - the requirement has been fulfilled (all decision problems related to it have been resolved).

4) Decision problem class represents architectural problems that have to be solved. It can be in one of the four states, which represent the stages of the problem's life cycle: defined - indicates a newly defined problem, being solved - the problem has been created, but it has not been resolved yet, resolved - problem has been resolved, requires reassessment - indicates that solution, or the occurrence of other problem, requires reconsidering an already resolved problem.

5) There are three types of relations that can link different decision problems: leadsTo, constrains and decomposesInto - compare section III.C.

6) The key element of the model of the service-oriented system is the Business Process, which identifies the 
system and allows navigation to all other elements of the model. The change of any element of the system, e.g. the change of one service, creates a new system that consists of new instances of all elements: Business process, Activities, Services, Service components and Operational components. Each Decision problem relates to a specific, currently considered, instance of the system and is indicated in the model by the input relation. The Business process identified by the input relation does not have to be identical to the initial process (associated by the old relation with the request for change). If the decision is made as the $\mathrm{n}$ in turn then the previous $\mathrm{n}-1$ decisions may already have modified this process.

7) There can be many alternative Solutions to a given Decision problem. Each of these solutions may create a new instance of a given Business process and its underlying components (services, service components, operational components). This is reflected by the output relation connecting the Solution and Business process classes. The analysis of a given solution can be in one of the four states: defined - assigned immediately after creating an element; feasible - indicates a solution meeting all the requirements, infeasible - indicates a solution that does not meet at least one of the requirements, chosen - indicates the finally selected solution.

8) Each of the alternative Solutions is supposed to be assessed in terms of its Pros and Cons. They are described by: a textual description; significance: low, medium or high; related requirements. The latter is optional and indicates: in case of cons - the requirements that cannot be met because of a given cons; in the case of pros the requirements whose fulfilment is guaranteed by the given pros.

9) Decision maker class represents the architects that resolve a Decision problem.

In practice, the MAD4SOA models should be created with the use of a diagrammatical notation summarised in Fig. 3. It is an extension of our previous MAD notation, made in order to capture the additional elements necessary to document the evolution of SOA systems.

Similarly to MAD, MAD4SOA notation comprises two types of diagrams: an Architecture Decision Relation Diagram (ADRD) and an Architecture Decision Problem Map (ADPM). ADRD represents the identified decision problems, while ADPM models an individual decision problem. Despite obvious similarities, there are many extensions made to MAD diagrams, aimed at including all the elements of the MAD4SOA model. The ADRD diagram now includes symbols that represent requests for change, requirements and business processes. As well as solutions of decision problems that produce a new release of the system. The ADPM diagram can now contain more than one decision problem. This is necessary in cases of a problem decomposition, as well as if a relation between the solutions to various problems has to be shown.

\section{Relations}

The MAD4SOA model comprises a set of relations that may be used in order to capture the real-world relations that may exist between: decision problems, decision problems and requirements, decision problems and solutions of other problems, as well as between the solutions of different decision problems. For the purpose of the definitions given below, let: $R$ - denote a set of requirements, $P$ - a set of architectural problems, and $A$ - a set of considered solutions (alternatives).

The motivates relation:

$$
\text { motivates } \subseteq R \times P
$$

It connects the requirements with the decision problems that have to be solved in order to meet the requirements.

For example: take a requirement to implement a new functionality of verifying a personal ID number. To fulfil this requirement, two decision problems have to be solved: "Which service should be used in order to verify the personal ID number?" and "In which step of the business process does this service have to be invoked?".

\section{The leadsTo relation:}

$$
\text { leadsTo } \subseteq P \times P
$$

In many cases, one decision problem implies the need to resolve another problem. Such problems should be connected with leadsTo relation.

For example: The problem of "choosing webservice implementation technology" makes it necessary to resolve a problem concerning the choice of the runtime environment for our webservices (it could be one of many application servers available on the market, or the application server already used by the corporate systems). Therefore the problems "Which type of webservices?" and "Which runtime environment?" are connected by the leadsTo relation.

Properties: The leadsTo relation is irreflexive, antisymmetrical and transitive. Therefore, this is a relation of acute partial order in the set of problems $\mathrm{P}$.

The decomposesInto relation:

$$
\text { decomposesInto } \subseteq P \times P
$$

It captures the decomposition of a given decision problem into several sub-problems. In practice, there are often cases, in which in order to solve a complicated decision problem, it is necessary to decompose it into several smaller sub-problems. The decomposed problem can be assumed as resolved only if all its sub-problems have been resolved.

For example: the problem of "choosing the software implementation technology" may comprise two sub-problems, namely: "choice of a programming language?" and "Choice of a mechanism of communication with the database?".

Properties: The decomposesInto relation is reflexive, antisymmetrical and transitive. Therefore, this is a relation of acute partial order in the set of problems P. There must be no cycles in the graph of this relation. 


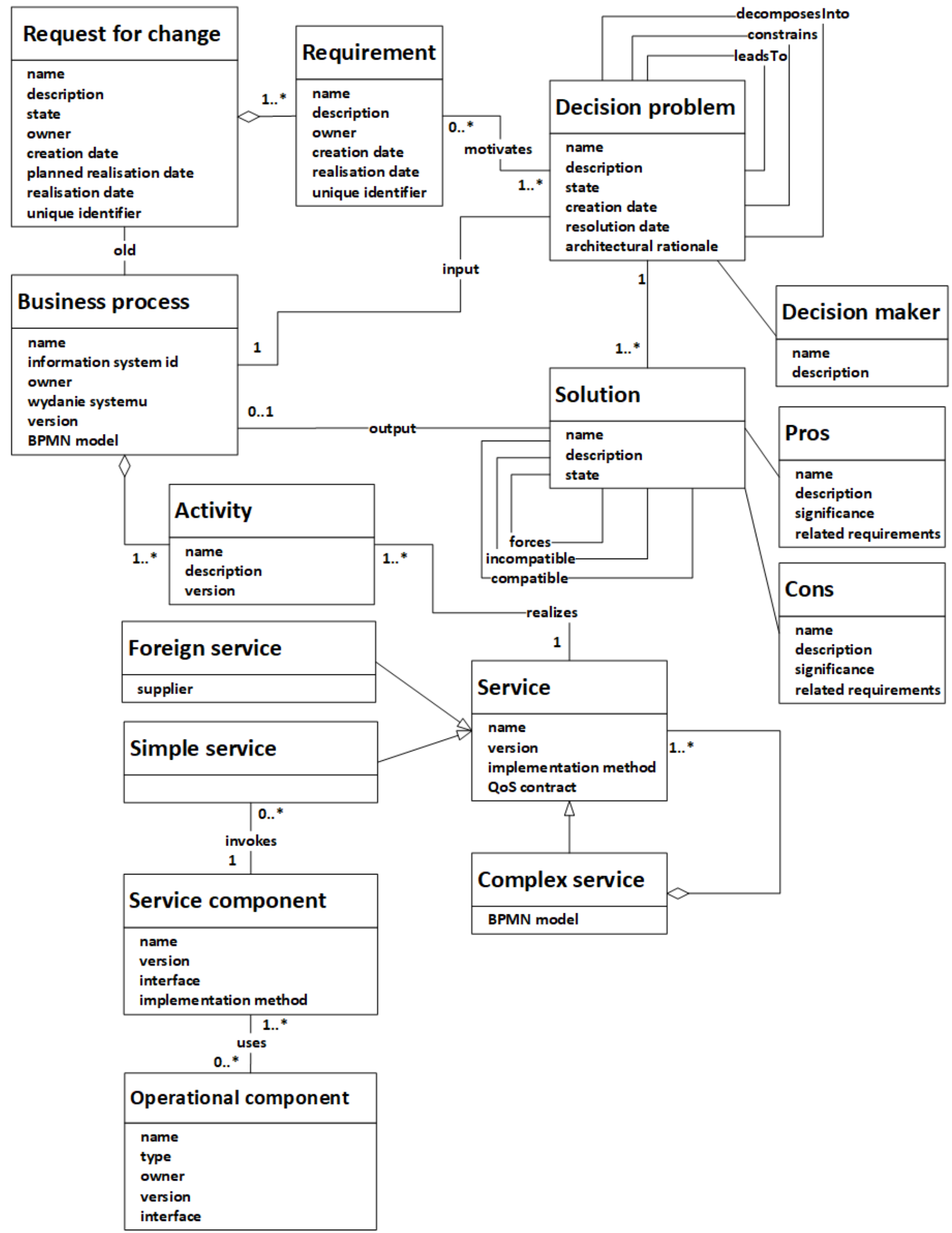

Fig. 2. Model of architectural decisions in the evolution of a service-oriented system. 


\section{The constrains relation:}

$$
\text { constrains } \subseteq P \times P
$$

There are often situations in which the solution of a given decision problem limits the scope of possible solutions to another problem. This is captured by the constrains relation.

For example: take two decision problems: "the problem of database selection" and the problem of "choosing the mechanism of data replication from the selected database to the MS SQL Server 2014 database". The choice of the Oracle $12 \mathrm{c}$ database will exclude the use of the replication mechanism provided with the MS SQL Server 2014 database, because MS SQL Server 2014 does not support replication from Oracle version $12 \mathrm{c}$. Therefore, there is a constrains relation between these problems.

Properties: The constrains relation is irreflexive and transitive. There must be no cycles in the graph of this relation.

\section{The compatible, incompatible and forces relations:}

$$
\begin{gathered}
\text { compatible } \subseteq A \times A \\
\text { incompatible } \subseteq A \times A \\
\text { forces } \subseteq A \times A
\end{gathered}
$$

In many cases, some solutions of different problems can exist together (they are compatible) or they cannot (they are incompatible) in the system architecture. Sometimes the relation between solutions is even stronger, so that choosing a certain solution simply forces us to choose a specific solution to another problem.

For example: take the problems of choosing the system implementation technology and selecting a specific application server that will be the execution environment for the designed software.

In the case of choosing J2EE as the implementation technology, we can choose both Jboss and Weblogic as the application server, because both of them support J2EE technology. There is a compatible relation between the J2EE and the Jboss solutions and Weblogic solution.

In the case of choosing J2EE technology, we cannot choose the IIS application server, because IIS does not support J2EE technology. There is an incompatible relation between the J2EE and IIS solutions.

In the case of choosing .NET as the implementation technology, we then have to choose IIS as the application server, because only this application server supports .NET technology. Therefore there is the forces relation between the .NET solution and the IIS solution.

Properties: The compatible relation is reflexive and symmetric. The incompatible relation is irreflexive and symmetric. The forces relation is irreflexive and antisymmetric.

\section{Integrity constrains of the model}

The relations between the elements of the MAD4SOA model of architectural decisions are not independent of each other. Some relations between decisions can exist at the same time, while others cannot. The integrity constrains make it possible to discover potential flaws in an instance of the MAD4SOA model crafted by an architect. Fourteen integrity constrains for the MAD4SOA model are presented below.

Integrity constraint 1: The decomposesInto and the leadsTo relations are mutually exclusive. The decomposed decision problem can only be solved when all subsequent problems raised as a result of its decomposition have been resolved. This would be superfluous to treating the problems resulting from the decomposition of a given problem as being enforced by the decomposed problem.

Integrity constraint 2: The decomposesInto and the constrains relations are mutually exclusive. The decomposed decision problem can only be solved if all its sub-problems are resolved. The solution of the decomposed problem is the superposition of the solutions to the sub-problems. Therefore, the solution to the decomposed problem cannot constraint the scope of the solutions to sub-problems.

Integrity constraint 3: If there is a constrains relation between two decision problems, then there is at least one pair of their solutions that are incompatible together (they are in an incompatible relation). The above property results from the meaning of the constrains relation. If problem A limits the set of solutions available to problem $\mathrm{B}$, it means that there must be solutions to problem $\mathrm{B}$ that are incompatible with one or more solutions to problem A.

Integrity constraint 4: The compatible and the incompatible relations are mutually exclusive. It is impossible for two solutions to be both compatible and incompatible at the same time.

\section{Integrity constraint 5:}

$$
\text { forces } \subseteq \text { compatible }
$$

If a certain solution to one problem forces the adoption of a specific solution of another problem in the same system, then the consistency of the system design requires that both solutions can coexist with each other.

Integrity constraint 6: The forces and the incompatible relations are separable. Because the forces relation is a subset of the compatible relation, it means that the forces and the incompatible relations are also mutually exclusive.

Integrity constraint 7: The forces relation cannot occur between solutions of the same decision problem. The next rules define the meaning of the states of the individual model elements.

Integrity constraint 8: The requirement may change its state to completed only if all related decision problems are in the resolved state. The requirement is considered as met only if it is possible to resolve all the problems connected with this requirement. That means that all decision problems arising as a result of this requirement will be resolved.

Integrity constraint 9: The RFC can go into the completed state only if all the requirements associated with it are in the completed state. The request for change can only be considered complete once all its architecturally significant requirements have been met. 
Symbols representing Decision Problems and their possible states:

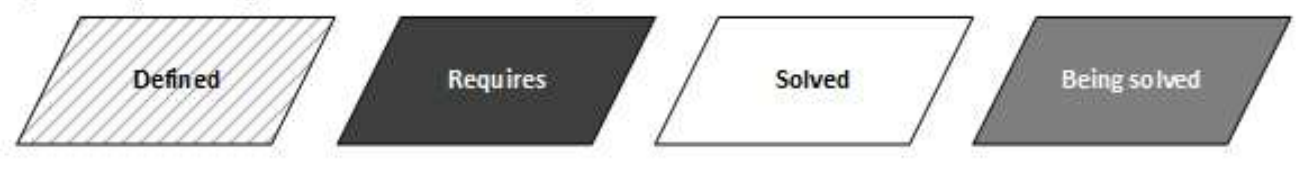

Symbols representing Solution of the problem and their states:

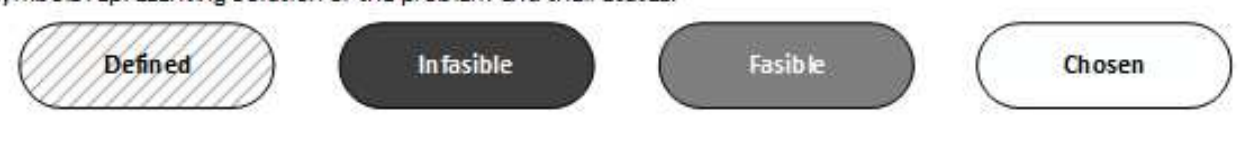

Symbols representing Cons of a given Solution:
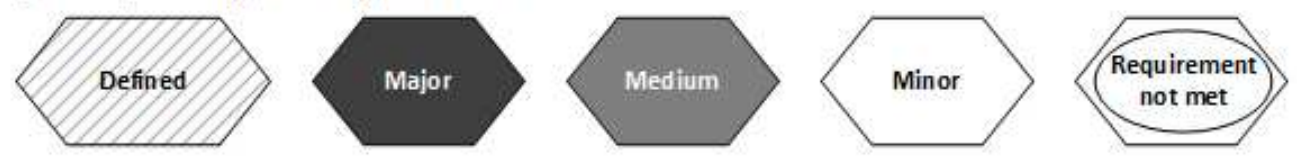

Symbols representing Pros of agiven Solution:
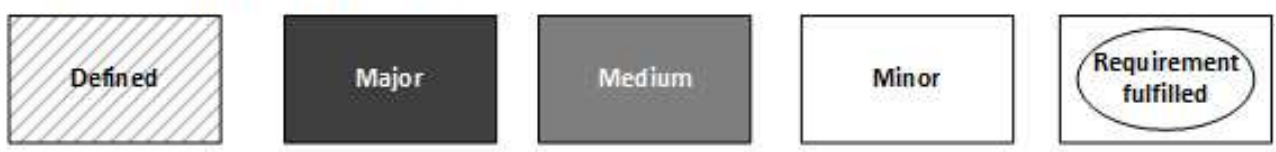

Symbols representing Request for change (RFC) and their possible states:
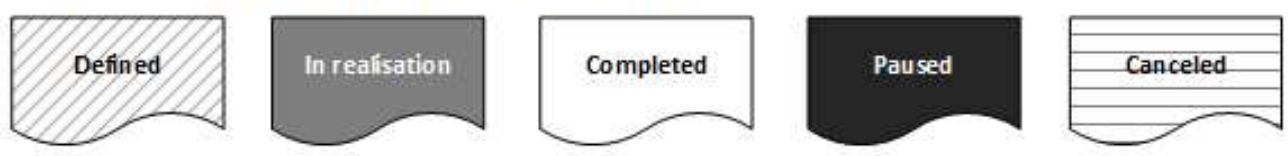

Symbols representing Requirements ant their possble states

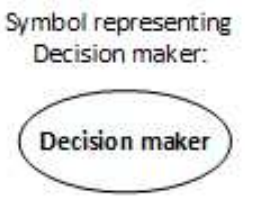

Symbol representing
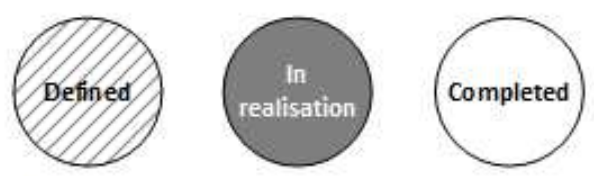

Business process:

Graphical repr esentation of relations between elements of the modet
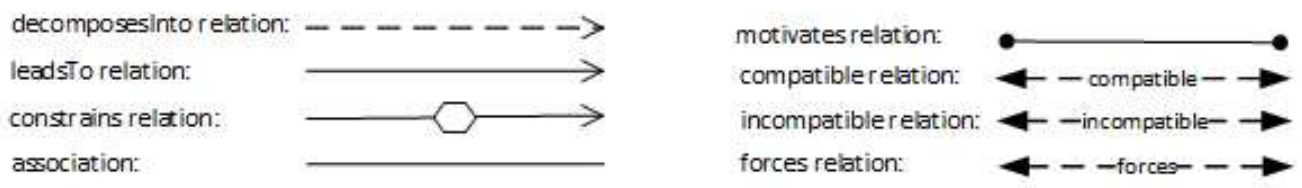

Fig. 3. The notation representing components of the MAD4SOA model.

Integrity constraint 10: At most, one solution of a decision problem can assume the chosen state. The system design must be unambiguous. That means that for each considered decision problem there must be only one clearly indicated chosen solution.

Integrity constraint 11: The decision problem may go into the resolved state only if one of its solutions assumes the chosen state.

Integrity constraint 12: If at least one cons of a solution goes into the requirement not met state, then this solution cannot be selected as the resolution of the decision problem, and should therefore go into the infeasible state.

Integrity constraint 13: There must be no incompatible relation between any two solutions in the chosen state (these solutions concern different problems). All resolutions of decision problems must coexist with each other in the same system to ensure system design consistency.

Integrity constraint 14: If there is a forces relation between the two solutions, then if one of them is chosen, the other must also be selected. Some solutions of decision problems cannot exist independently in the system.

\section{The CAse Study - VAlidation of MAD4SOA MODEL}

This section presents a case study illustrating the use of the MAD4SOA model for capturing the evolution of a real service-oriented system. This case study is based on the clearing system operating in the Polish banking system. The system has been designed to settle instant transfers carried out between two banks. The clearing system verifies each payment order, confirms the consent of both banks to carry out the transaction and registers the change in the account balances 
of both banks and in the Central Bank system. Performing these activities requires the interaction of the clearing system with the systems of banks participating in a transaction (the ordering bank and the bank receiving the transfer) and with the system of the Central Bank. The evolution process of the system has produced six releases of the system in several evolution steps:

- Creation of functionality of instant payments between commercial banks as the first release

- Adding instant payments to the Central Bank (tax payments) as the second release

- Adding a simple functionality of complaints as the third release

- Adding a Back Office module as the fourth release

- Adding postponed payments as the fifth release

- Removing one type of tax payment as the sixth release.

The lifecycle of the system has not been finished yet.

As a part of the example, the evolution of the Back Office module of the clearing system has been presented. This module enables customer complaint handling, as well as the parameterisation and monitoring of the system. These activities were carried out manually by making changes to the tables in the database using previously prepared scripts in earlier stages of the life cycle of the system. Next, the Back Office has been implemented to enable the automation of these activities.

The complaint handling process is the most important functionality of the Back Office application. Complaints are generated if the bank ordering the transfer does not have sufficient funds on the internal account, or the bank receiving the transfer does not confirm the transfer. If necessary, the administrator can make a decision and manually modify the transaction state.

Several decision problems had to be resolved by the architect during the design of the Back Office functionality during both evolution steps. The ADRD diagram that presents the decision problems solved by the architect is presented in Figure 4. The example of the ADPM diagram presented in Figure 5 describes how the problems identified during this evolution step (step 4) have been resolved. The most important is the evolution of the decision problem: "How to implement the complaint handling functionality?". This problem had been resolved in the current version as "Using predefined scripts" during the previous evolution step (step 3). It had to be revised due to implementing a new RFC during the next evolution step (step 4). Both solutions to this problem produced new releases of the system in subse-quent evolution steps. It is represented by output relation linking solutions and in-stances of business processes.

The business process representing the third release of the system and a Request for Change describing changes implemented in the fourth evolution step are connected by the old relation. It means that this RFC applies to the modification of the third release of the system. The business process representing the third release of the system is also linked with the "How to implement the complaint handling functionality?" decision problem resolved in the fourth evolution step by the input relation. This means that the third release of the system has been modified and transformed to the fourth release. Additionally, this ADRD diagram represents several decision problems that have been resolved during the fourth evolution step. The detailed process of solving those problems is presented in Figure 5.

\section{DISCUSSION}

The MAD4SOA model, which is the core contribution of this paper, has been tailored to the needs of documenting and supporting the engineering in the course of evolution of service-oriented systems. It provides for linking decisions made during different evolution steps, which makes it possible to trace the sequence of actions taken by architects during the development of subsequent releases of the system. An important feature of the proposed model is the inclusion of request for change document and its requirements as a model's entity. This brings the model closer to the real-world practice. All these unique features of MAD4SOA model constitute the core of our contribution.

Naturally, there are many similarities between proposed models and alternative architecture decision models presented in Section II, which is inevitable. They concern mainly:

- the model of architectural decisions, which can be traced back to our earlier research on MAD model [10] as well as to classical works on architecture decisions [5];

- some relations (e.g. decomposesInto) and integrity constraints (e.g. integrity constraint No. 6) can be easily traced back to the RADM model [6];

- tracability machanism linking diagramatical architectural models (e.g. in BPMN) with architectural decisions can also be found in [17].

An added value that MAD4SOA delivers is also an intuitive diagrammatic notation for representing architectural decisions, which is similar to popular mind maps. In contrast to RADM, we do not introduce any scheme or mechanism for classifying architectural decisions similar to topic groups or levels. Our internal experiments, have shown that this in too many cases leads rather to confusion than to clarity. In our model all the changes are linked to business processes, which they may finally affect.

The main limitation of the proposed approach results from the complexity of the architecture-decision model and its associations with the models of SOA system (e.g. business process models in BPMN), which may hinder its use. Naturally, more extensive evaluation and tool support is necessary.

\section{Conclusion}

The complete model supporting the capturing of architectural decisions during the evolution of a service-oriented system has been proposed. It combines an architectural decision model and a model of a service-oriented system. Thanks to this, MAD4SOA supports capturing architectural knowledge created during the evolution of a service-oriented system. The use of the model has been validated on the evolu-tion of a 


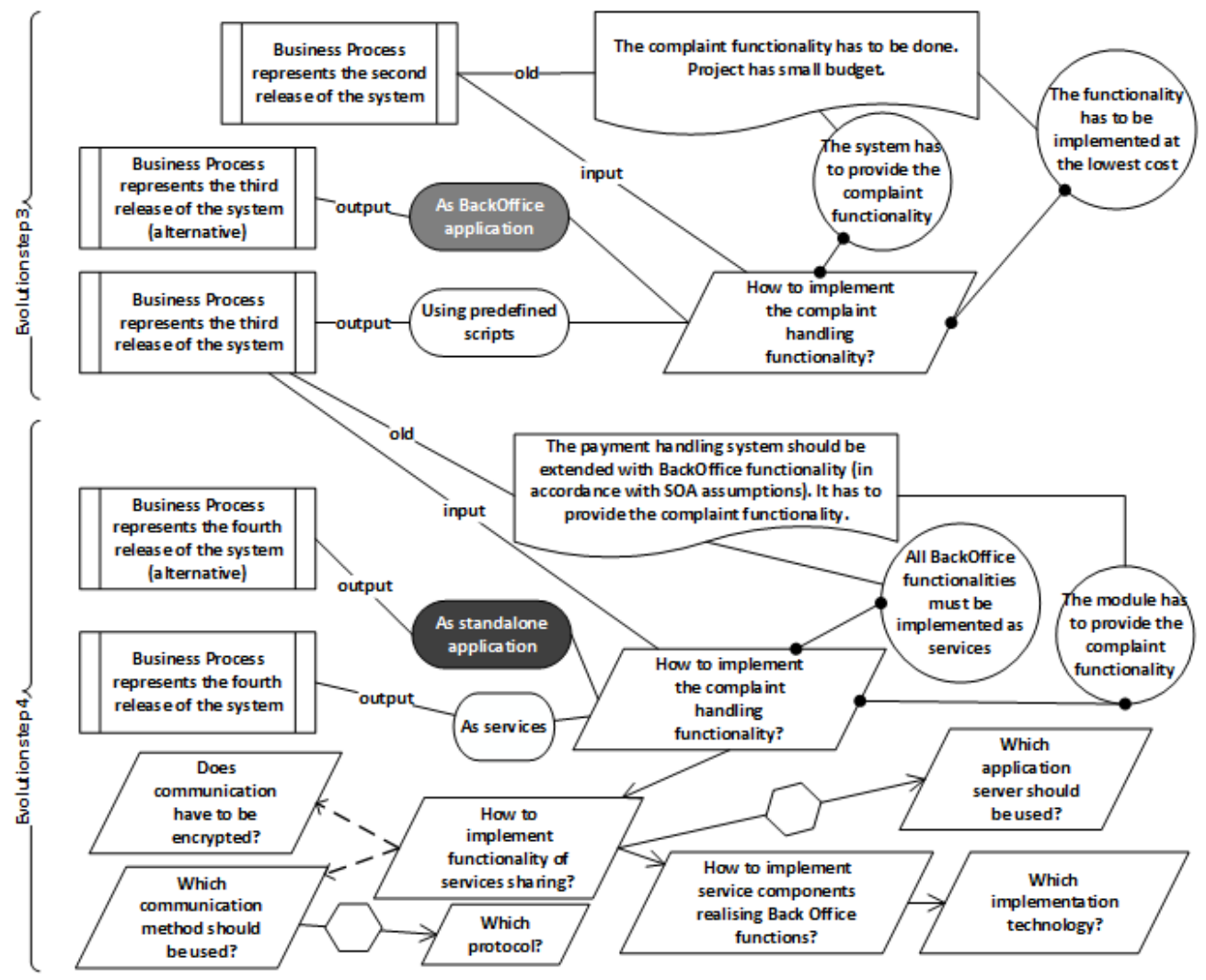

Fig. 4. The ADRD diagram presenting problems resolved during the design of the new release.

real service-oriented system operating in the Polish banking system.

Further work shall include defining formal semantics of MAD4SOA model and developing supporting tools.

\section{REFERENCES}

[1] M. Bell, "SOA Modeling Patterns for Service-oriented Discovery and Analysis," Wiley and Sons, 2010.

[2] M. Ali Babar, et al.,"Architecture knowledge management. Theory and Practice," Springer - Verlag Berlin Heidelberg, 2009.

[3] J. Tyree, A. Akerman, "Architecture Decisions: Demystifying Architecture," IEEE Software, vol. 22, iss. 2, 2005, pp. 19-27.

[4] ISO/IEC, "ISO/IEC/IEEE 42010:2011: Systems and software engineering - Architecture description," ISO/IEC, 2011.

[5] A. Jansen, J. Bosch, "Software Architecture as a Set of Architectural Design Decisions," 5thWorking IEEE/IFIP Conference on Software Architecture, 2005, pp. 19-27.

[6] O. Zimmermann, et al., "Managing architectural decision models with dependency relations, integrity constraints, and production rules," Journal of Systems and Software, vol. 82, no. 8, 2009, pp. 1249-1267.

[7] P. Kruchten, P. Lago, H. van Vliet, "Building up and reasoning about architectural knowledge," QoSA, 2006.

[8] P. Kruchten, "An Ontology of Architectural Design Decisions," Proc. of 2nd Groningen Workshop on Software Variability Management, 2004, pp. 54-61.

[9] The Open Group, "The Open Group Architecture Framework (TOGAF®) Version 9.1," The Open Group, http://pubs.opengroup.org/ architecture/togaf9-doc/arch/ 2018

[10] A. Zalewski, S. Kijas, D. Sokołowska, "Capturing Architecture Evolution with Maps of Architectural Decisions 2.0," ECSA 2011 Lecture Notes in Computer Science, vol. 6903, 2011, pp. 83-96.

[11] N. B. Harrison, P. Avgeriou, U. Zdun, "Using Patterns to Capture Architectural Decisions," IEEE Software, vol. 24, no. 4, 2007, pp. 3845 .
[12] M. Shahina, P. Lianga, M. Ali Babar, "A systematic review of software architecture visualization techniques," The Journal of Systems and Software, vol. 94, 2014, pp. 161-185.

[13] T. Erl, "Service-Oriented Architecture: Concepts, Technology, and Design, Upper Saddle River," Prentice Hall PTR, 2015.

[14] A. Arsanjani, S. Ghosh, A. Allam, T. Abdollah, S. Ganapathy, K. Holley, "SOMA: A method for developing service - oriented solutions," IBM Systems Journal, vol. 47, no. 3, 2008, pp. 377-396.

[15] M. P. Papazoglou, W. J. van den Heuvel, "Service-oriented design and development methodology, International Journal of Web Engineering and Technology," IJWET, 2006.

[16] R. Capilla, F. Nava, J. C. Dueñas, "Modeling and Documenting the Evolution of Architectural Design Decisions," Proc. 2nd Workshop Sharing and Reusing Architectural Knowledge Architecture, 2007.

[17] R. Capilla, O. Zimmermann, U. Zdun, P. Avgeriou, J. M. Küster, "An Enhanced Architectural Knowledge Metamodel Linking Architectural Design Decisions to other Artifacts in the Software Engineering Lifecycle," ECSA 2011 Lecture Notes in Computer Science, vol. 6903 2011, pp. 303-318

[18] O. Zimmermann, J. Grundler, S. Tai, F. Leymann, "Architectural Decisions and Patterns for Transactional Workflows in SOA," ICSOC 2007 Lecture Notes in Computer Science, vol. 4749, 2007, pp. 81-93.

[19] O. Zimmermann, "Architectural Decisions as Reusable Design Assets," IEEE Software, vol. 28, 2011, pp. 64-69.

[20] M. Nowak, C. Pautasso, O. Zimmermann, "Architectural decision modeling with reuse: challenges and opportunities," Proceedings of the 2010 ICSE Workshop on Sharing and Reusing Architectural Knowledge, 2010, pp. 13-20

[21] C. Pautasso, O. Zimmermann, F. Leymann, "Restful web services vs. "big"' web services: making the right architectural decision," Proceedings of the 17th international conference on World Wide Web, 2008, pp. 805-814.

[22] X. Wang, N. Ali, I. Ramos, R. Vidgen, "Agile and Lean Service-Oriented Development: Foundations, Theory, and Practice," IGI Global, 2012. 


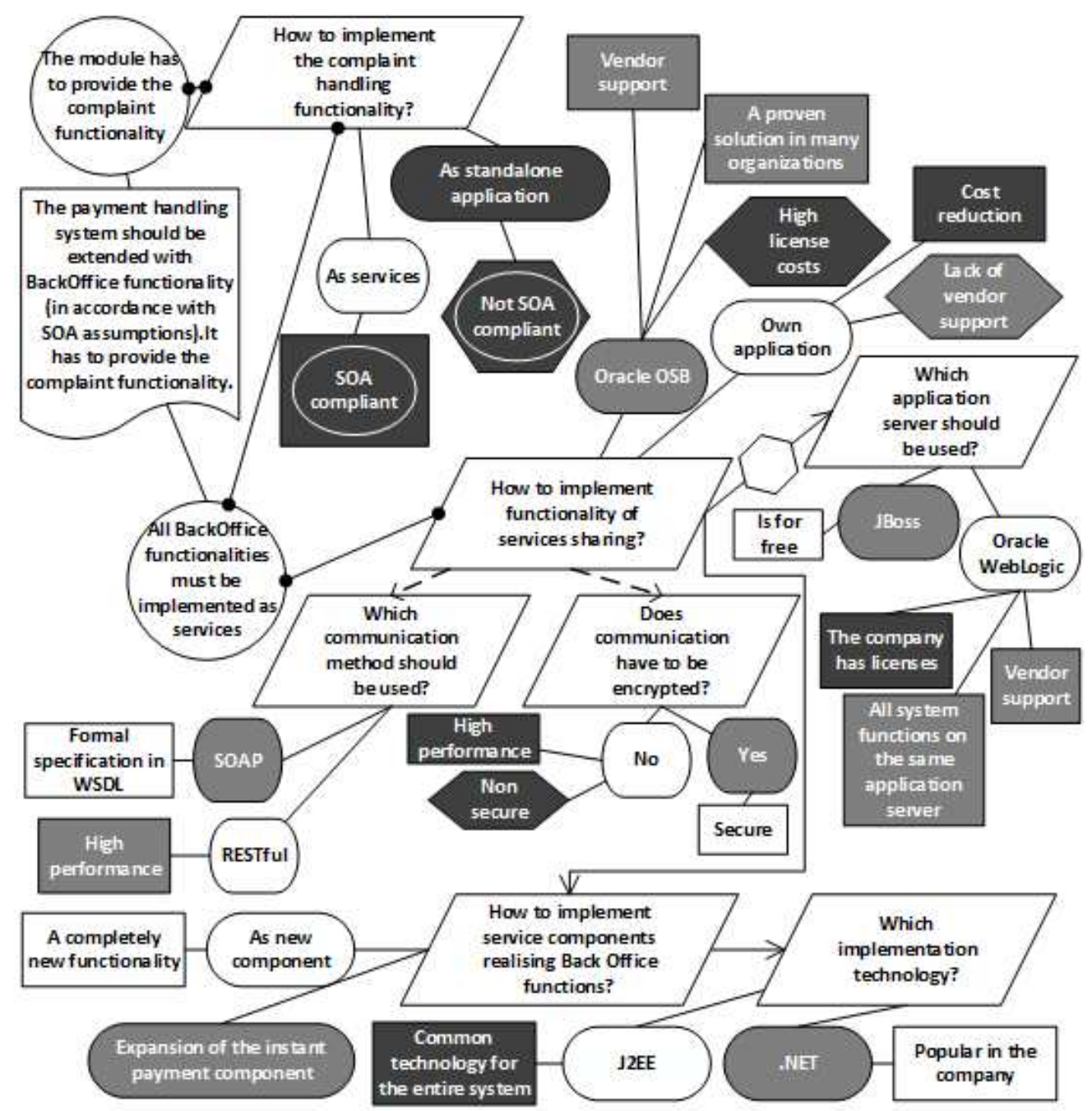

Fig. 5. The ADPM diagram presenting how to be resolve the problems arising during the design of the new system release. 


\section{Author Index}

Apostolov, Stoyan

. 17

Dimitrov, Yuri

13,17

Dimov, Ivan

$13,17,21,25,29$

$\mathrm{F}_{\text {idanova, Stefka }}$

Franczyk, Bogdan

$13,17,21,25,29$

Gotsov, Tsvetomir

Goullieux, Adeline

$\mathrm{H}_{\text {ajder, Mirosław }}$

Hajder, Piotr

Hifi, Mhand

J arkovský, Jiří

Karolyi, Matěj

Kijas, Szymon ....

Kolev, Tsvetelin....

Komenda, Martin

Krajsic, Philippe

Landaverde, Silvia Torres

Liput, Mateusz. ...
Militzer-Horstmann, Carsta ..................... 57

Miryanov, Radan.............................13

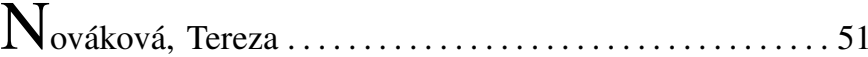

Nycz, Mariusz...................................... 35

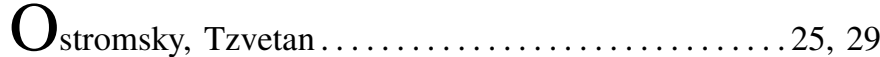

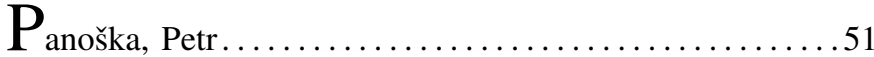

Poryazov, Stoyan ..................... 13, 17, 21

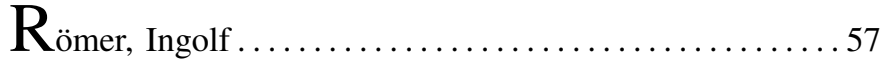

$\mathbf{S}_{\text {adeghsa, Shohre } \ldots \ldots \ldots \ldots \ldots \ldots \ldots \ldots \ldots \ldots}$

Sazonova, Polina............................. 45

Schieck, Martin.............................57

Todorov, Venelin.................3, 13, 17, 21, 25, 29

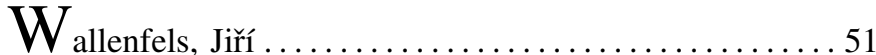

Zalewski, Andrzej .........................6 67 